\title{
Der Einfluss von Persönlichkeitsvariablen auf den Sunk-Cost-Effekt: Eine kritische Replikation und Erweiterung
}

\author{
Dissertation \\ zur Erlangung des wirtschaftswissenschaftlichen Doktorgrades \\ der Wirtschaftswissenschaftlichen Fakultät der Universität Göttingen
}

vorgelegt von

Sören Warnecke

aus Holzminden

Göttingen, 2017 
Erstgutachter:

Zweitgutachter:

Tag der mündlichen Prüfung:
Prof. Dr. Stefan Schulz-Hardt

Prof. Dr. Klaus Möller

09. März 2017 


\section{Danksagung}

Die Abgabe der vorliegenden Dissertation markiert für mich den Abschluss einer Lebensphase, die von Höhen und Tiefen geprägt war. An dieser Stelle möchte ich mich zunächst bei den Menschen bedanken, die mich in dieser Zeit maßgeblich begleitet und unterstützt haben.

Allen voran danke ich meinem Doktorvater Prof. Dr. Stefan Schulz-Hardt, dass er mir die Gelegenheit gegeben hat, in dem Forschungsbereich meiner Wahl zu promovieren und dass ich mich jederzeit mit Fragen und Problemen an ihn wenden konnte. Seine Geradlinigkeit und Präzision gaben mir Orientierung im Verlauf der Promotion und werden mir immer ein Vorbild für meine eigenen Handlungsweisen sein. Zudem waren es seine Vorlesungen im Rahmen meines BWL-Studiums, die überhaupt erst mein Interesse an der Wirtschaftspsychologie und der empirischen Forschung geweckt haben und bei mir den Wunsch auslösten, einmal selbst in diesem Bereich aktiv zu forschen.

Zugleich danke ich meinem Zweitgutachter Prof. Dr. Klaus Möller, dass er mir nach meinem Studium einen direkten Einstieg in die empirische Forschung an der Schnittstelle zwischen der Betriebswirtschaft und der Psychologie ermöglicht hat. Ich habe in dieser Zeit viel über effizientes Projektmanagement gelernt, was mir seitdem immer wieder in den verschiedensten Bereichen des täglichen Lebens zugutekommt.

Vor allem aber danke ich meinen Eltern Willi und Sigrid Warnecke, die mit mir gemeinsam durch alle Höhen und Tiefen meines Lebens gegangen sind. Sie und meine Freundin Katharina Knopke stellten nicht nur im Verlauf der Promotion meinen größten Rückhalt dar und ich wünsche mir, dass ich mit ihnen noch viele weitere Lebensabschnitte erleben werde. 


\section{Zusammenfassung}

Der Sunk-Cost-Effekt beschreibt die irrationale Tendenz, Entscheidungen über die Fortsetzung eines Unterfangens oder die Nutzung einer Sache davon abhängig zu machen, ob und in welchem Umfang hierfür bereits versunkene Kosten entstanden sind. Dieses kann in der Praxis kostspielige Konsequenzen nach sich ziehen. Eine Reihe empirischer Arbeiten legt jedoch nahe, dass bestimmte Persönlichkeitsvariablen darüber mitbestimmen, ob Entscheidungsträger mehr oder weniger anfällig für Sunk-Cost-Effekte sind. Hierbei handelt es sich um Handlungs- und Lageorientierung, Optimismus und Pessimismus, den regulatorischen Fokus: Promotion und Prevention sowie den Rational Thinking Style. Eine Berücksichtigung der entsprechenden Effekte könnte in der Praxis nützlich sein, z.B. indem gezielt Personen in Entscheidungspositionen eingesetzt werden, die aufgrund ihrer Persönlichkeit relativ robust gegenüber Sunk-Cost-Effekten sind. Das Ziel der vorliegenden Arbeit war daher, die entsprechenden Originalbefunde über diese Persönlichkeitsvariablen systematisch auf ihre Replizierbarkeit zu überprüfen und gegebenenfalls zu erweitern. Hierzu wurde sowohl eine Reihe direkter Replikationsstudien durchgeführt, die sich so nahe wie möglich an den jeweiligen Originalstudien orientierten, als auch eine integrative Replikationsstudie, in der alle Persönlichkeitsvariablen gleichzeitig erhoben wurden. Die zugrunde liegenden Originalbefunde konnten jedoch in keiner der durchgeführten Studien repliziert werden. Die Ergebnisse der vorliegenden Arbeit legen vielmehr nahe, dass in allen Fällen Zweifel an der Belastbarkeit der Persönlichkeitseffekte angebracht sind und es sich in der Praxis daher nicht anbietet, zur Vermeidung von Sunk-Cost-Effekten gezielt Entscheidungsträger anhand dieser Persönlichkeitsvariablen auszuwählen. Diese Doktorarbeit operiert thematisch an der Schnittstelle zwischen Psychologie und Wirtschaftswissenschaften und leistet einen fachübergreifenden Beitrag zur Aufdeckung nicht replizierbarer Befunde in der Entscheidungsforschung. 


\section{Inhaltsverzeichnis}

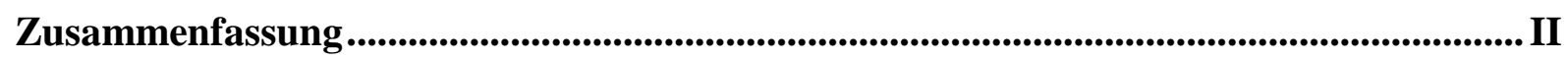

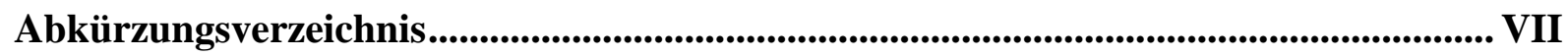

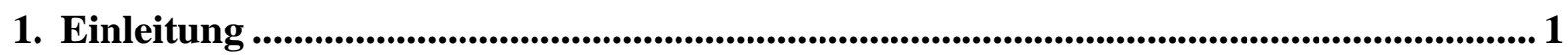

2. Theoretischer und empirischer Hintergrund....................................................................... 4

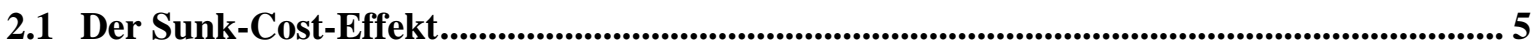

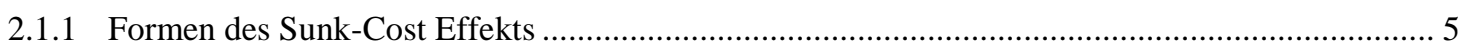

2.1.2 Empirie und Abgrenzung zu themenverwandten Konstrukten .......................................... 7

2.1.3 Operationalisierung in Sunk-Cost-Untersuchungen ...................................................... 9

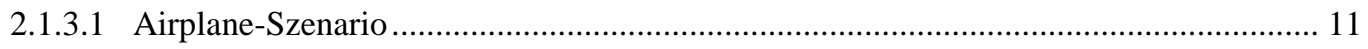

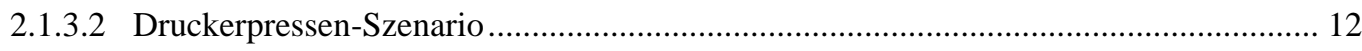

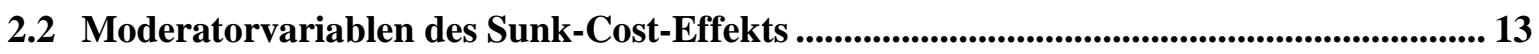

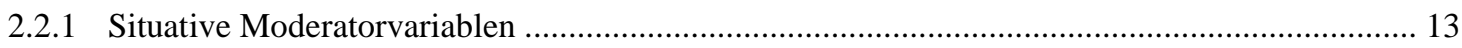

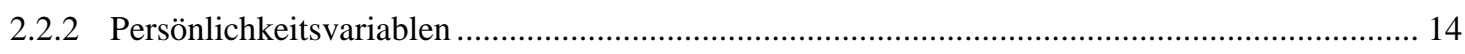

2.2.2.1 Handlungsorientierung und Lageorientierung ..................................................... 16

2.2.2.2 Optimismus und Pessimismus ........................................................................ 19

2.2.2.3 Regulatorischer Fokus: Promotion und Prevention ................................................ 22

2.2.2.4 Rational Thinking Style ................................................................................... 25

2.3 Replizierbarkeit empirischer Befunde............................................................................................. 28

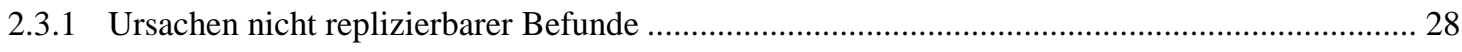

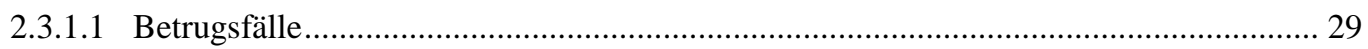

2.3.1.2 Questionable Research Practices und Confirmation Bias ..................................... 30

2.3.1.3 Statistische und menschliche Fehler .................................................................... 32

2.3.1.4 Publication Bias ............................................................................................... 34

2.3.2 Notwendigkeit zur Durchführung von Replikationsstudien ................................................. 35

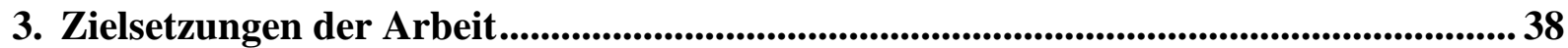




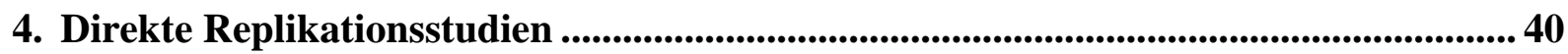

4.1 Handlungs- und Lageorientierung...................................................................................................... 41

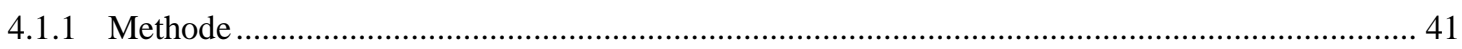

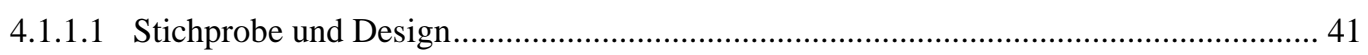

4.1.1.2 Durchführung und Material ..................................................................................... 41

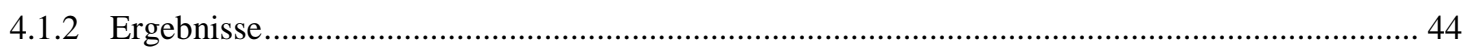

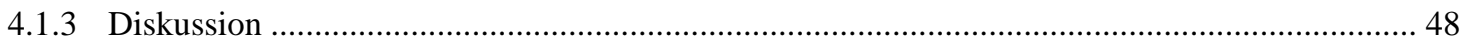

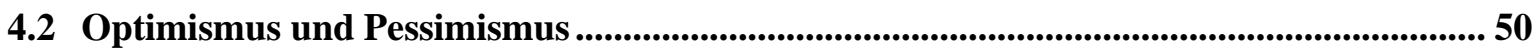

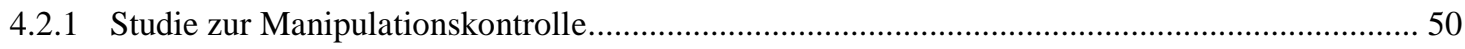

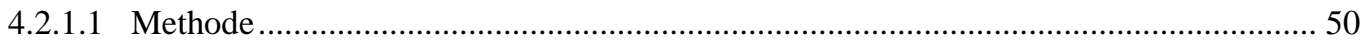

4.2.1.1.1 Stichprobe und Design ............................................................................................. 50

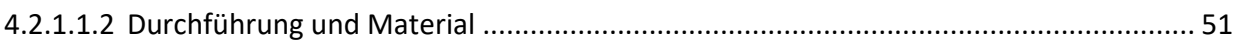

4.2.1.2 Ergebnisse

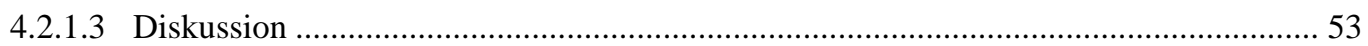

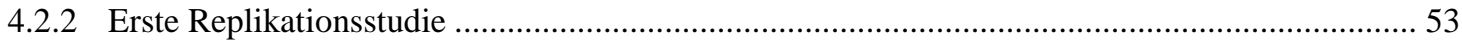

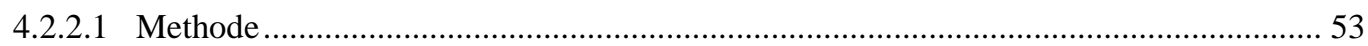

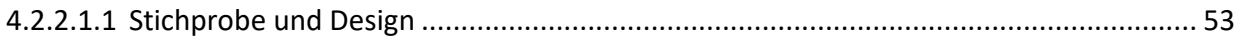

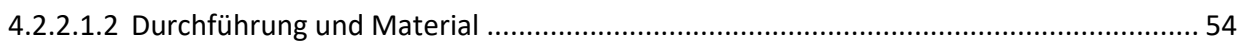

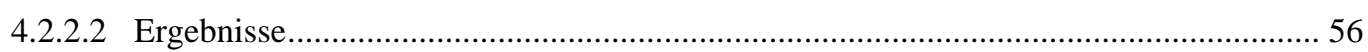

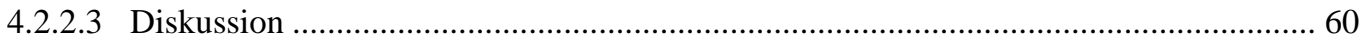

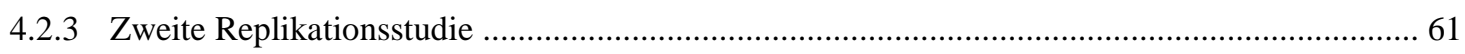

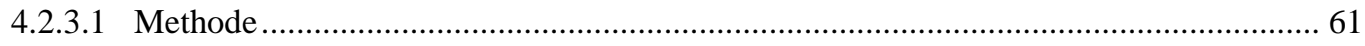

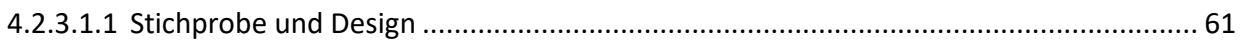

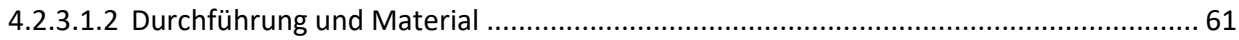

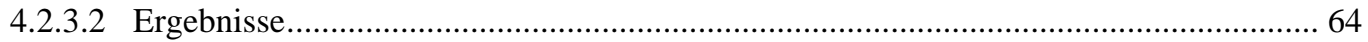

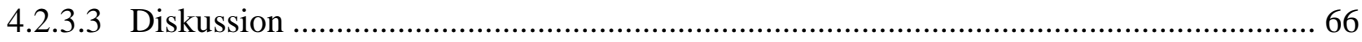

4.3 Regulatorischer Fokus: Promotion und Prevention .......................................................... 67

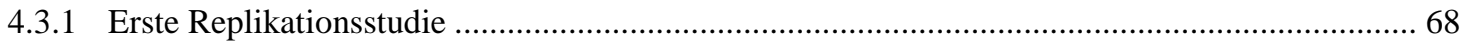

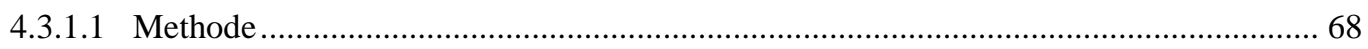

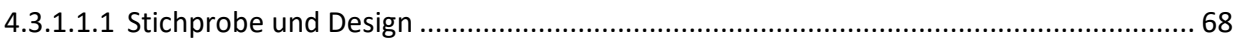

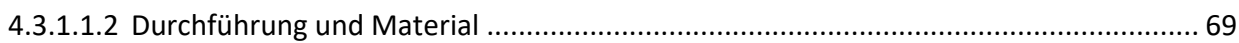




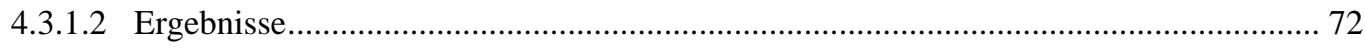

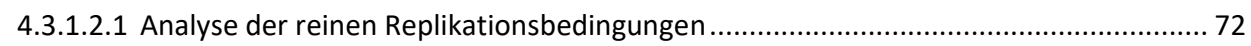

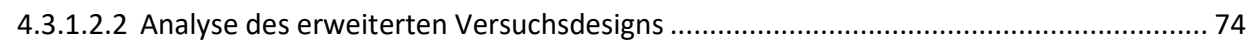

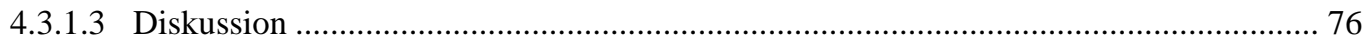

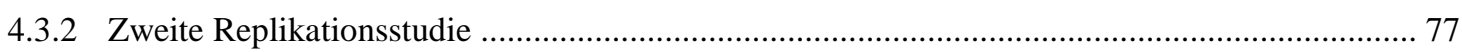

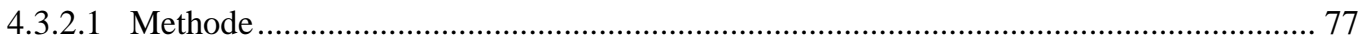

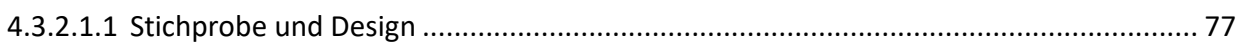

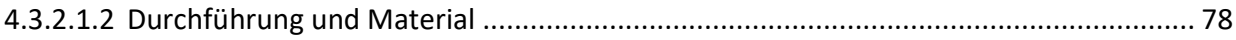

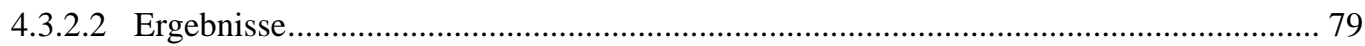

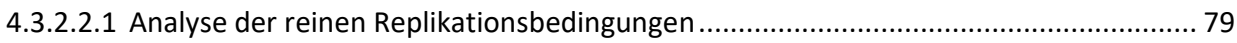

4.3.2.2.2 Analyse des erweiterten Versuchsdesigns ........................................................... 79

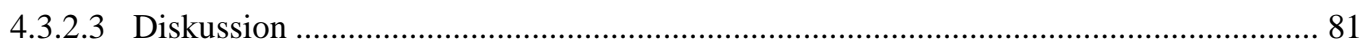

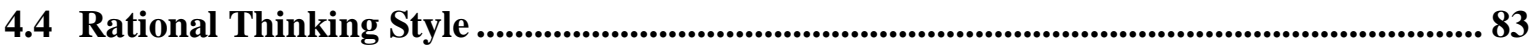

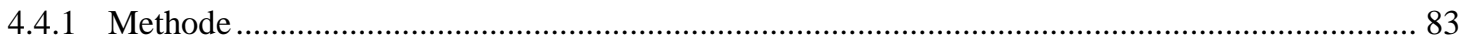

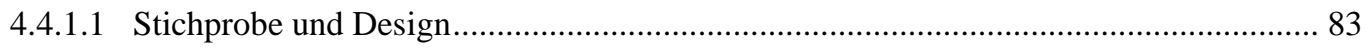

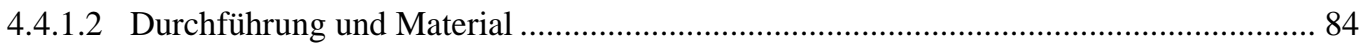

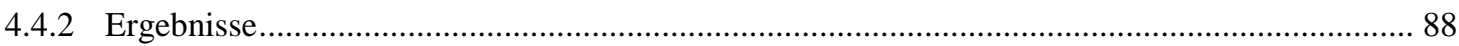

4.4.2.1 Analyse der reinen Replikationsbedingungen .............................................. 89

4.4.2.2 Analyse des erweiterten Versuchsdesigns ..................................................... 91

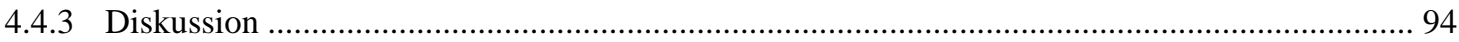

4.5 Fazit der direkten Replikationsstudien ......................................................................... 96

5. Integrative Replikationsstudie ...................................................................................................99

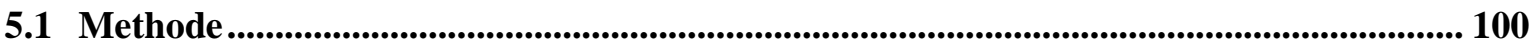

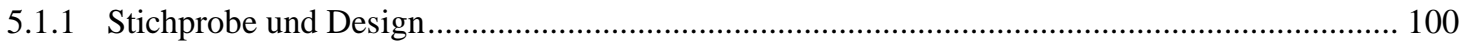

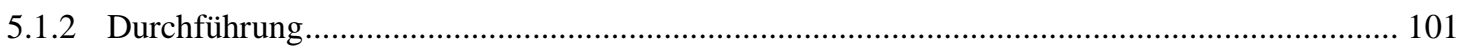

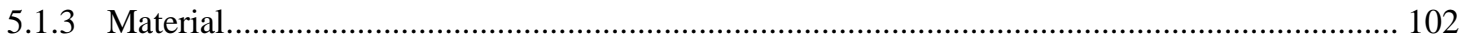

5.1.3.1 Erster Teil: Messung der Persönlichkeitsvariablen................................................ 102

5.1.3.1.1 Handlungs- und Lageorientierung ..................................................................... 103

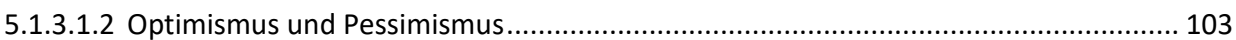

5.1.3.1.3 Regulatorischer Fokus: Promotion und Prevention .............................................. 103

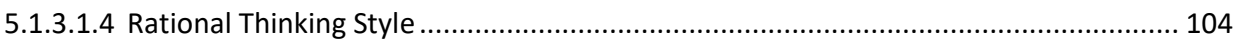


5.1.3.2 Zweiter Teil: Messung der Sunk-Cost-Neigung .............................................. 105

5.1.3.3 Dritter Teil: Abschlussfragebogen ................................................................... 106

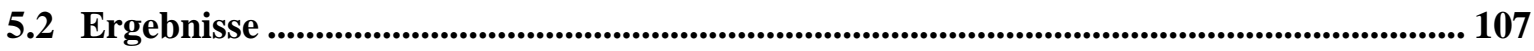

5.2.1 Einzelanalysen der Persönlichkeitsvariablen ....................................................... 108

5.2.1.1 Handlungs- und Lageorientierung ............................................................. 108

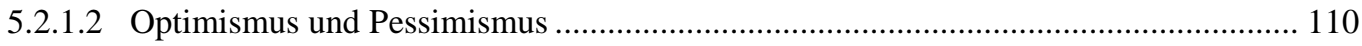

5.2.1.3 Regulatorischer Fokus: Promotion und Prevention ........................................... 113

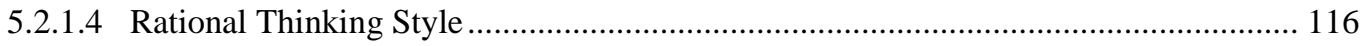

5.2.2 Integrative Analysen der Persönlichkeitsvariablen ....................................................... 119

5.2.2.1 Analysen des Airplane-Szenarios ................................................................ 120

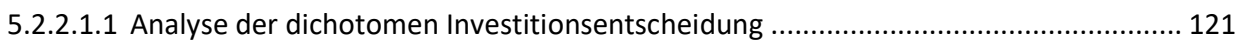

5.2.2.1.2 Analyse der prozentualen Investitionsbereitschaft............................................... 122

5.2.2.2 Analysen des Druckerpressen-Szenarios ..................................................... 123

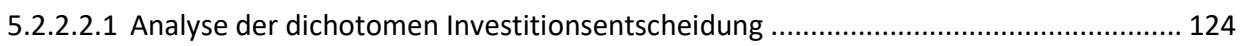

5.2.2.2.2 Analyse der prozentualen Investitionsbereitschaft............................................... 125

5.3 Diskussion..................................................................................................................... 126

6. Übergreifende Diskussion .......................................................................................................... 128

Literaturverzeichnis.........................................................................................................................VIII

Tabellenverzeichnis........................................................................................................................XXI

Abbildungsverzeichnis ..................................................................................................................XXIV

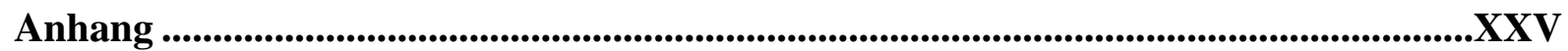




\section{Abkürzungsverzeichnis}

ACS-90

ANOVA

AO

AV

BWL

$\mathrm{CD}$

$\mathrm{EoC}$

HAKEMP-90

IE

IQ

JPSP

LOT-R

NBA

OP

QRP

RA

RE

$\mathrm{RF}$

RTS

$\mathrm{SC}^{(+)}$

$\mathrm{SC}^{(-)}$

SEK
Action Control Scale (engl. Version des HAKEMP-90)

Analysis of variances

Action orientation

Abhängige Variable

Betriebswirtschaftslehre

Compact Disc

Escalation of Commitment

Fragebogen zur Messung von Handlungs- und Lageorientierung

Interaktionseffekt

Intelligenzquotient

Journal of Personality and Social Psychology

Life-Orientation-Test (Revision)

National Basketball Association

Optimism and Pessimism

Questionable Research Practice

Rational Ability

Rational Engagement

Regulatory Focus

Rational Thinking Style

hohe bzw. vorhandene versunkene Kosten (Sunk Costs)

niedrige bzw. abwesende versunkene Kosten (Sunk Costs)

Schwedische Kronen

Die im Rahmen dieser Arbeiten verwendeten statistischen Kennwerte können bei Bedarf z.B. bei Bortz und Schuster (2010) nachgeschlagen werden. 


\section{Einleitung}

Der Sunk-Cost-Effekt ist ein klassischer psychologischer Entscheidungsfehler mit hoher wirtschaftlicher, gesellschaftlicher und individueller Relevanz. ${ }^{1}$ Er tritt auf, wenn Personen eine Entscheidung auf Basis bereits versunkener Kosten treffen, während sie sich ohne oder bei geringeren versunkenen Kosten, unter sonst gleichen Bedingungen, anders entschieden hätten (vgl. Arkes und Blumer, 1985; Thaler, 1980). Beispielsweise berichteten Arkes und Blumer (1985), dass Personen in einer Feldstudie, die einen höheren Preis für Tickets einer Theatersaison bezahlt hatten, häufiger Theatervorstellungen besuchten als Personen, die einen Rabatt auf den Ticketpreis erhalten hatten. Ähnliche Befunde berichteten Just und Wansink (2011) aus einer Feldstudie in einem All-You-Can-Eat Restaurant. Personen, die hierbei den vollen Preis für den Zugang zu einem Pizzabuffet bezahlt hatten, wiesen einen signifikant höheren Konsum von Pizzastücken auf als Personen, die nur den halben Preis zahlen mussten. Des Weiteren gibt es Nachweise für höhere Einsatzzeiten und längere Vereinszugehörigkeit von Basketballspielern in der NBA, je höher sie in der Draft Order standen, welche gleichzeitig mit höheren Spielergehältern für die Vereine einhergeht (Staw \& Hoang, 1995). Selbst die Löschung eines sorgfältig geschriebenen Textes, z.B. in einem Manuskript wie diesem, kann einem Verfasser aufgrund der bereits investierten Zeit schwerfallen (Hafenbrack, Kinias \& Barsade, 2014).

Gemäß der ökonomischen Theorie sollten zukunftsbezogene Entscheidungen hingegen immer durch Abwägung marginaler Größen getroffen werden, also durch die Bewertung zukünftiger Grenzkosten und Grenzerlöse (Thaler, 1985). Versunkene Kosten bieten keine Entscheidungsgrundlage, da ihr Auszahlungszeitpunkt bereits in der Vergangenheit liegt oder für die Zukunft fix determiniert ist. Diese Kosten sind unwiederbringlich „versunken“ und

\footnotetext{
${ }^{1}$ In der Literatur ist der Sunk-Cost-Effekt auch unter den Begriffen Sunk-Cost-Bias, Sunk-Cost-Fallacy (vgl. Hafenbrack, Kinias \& Barsade, 2014) oder Concorde Effect (Arkes \& Ayton, 1999) zu finden.
} 
können ex post weder verändert noch vermieden oder rückgängig gemacht werden (vgl. GroßSchuler, 2002; Krahnen, 1991; Pindyck \& Rubinfeld, 2009 oder Schaub, 1997). ${ }^{2}$

Die Erforschung des Sunk-Cost-Effekts operiert an der Schnittstelle zwischen der Psychologie und den Wirtschaftswissenschaften. Vor allem in der Wirtschaft sollte die Vermeidung von Sunk-Cost-Effekten eine hohe Priorität haben, da hierdurch verursachte Fehlentscheidungen Konsequenzen von hoher Tragweite mit sich bringen können. So wird es beispielsweise problematisch, wenn Entscheidungsträger aufgrund versunkener Kosten an fehlgehenden Projekten festhalten oder diese sogar noch intensivieren, sodass in der Folge objektiv gleichwertige oder sogar bessere Alternativen verworfen werden (vgl. Arkes \& Blumer, 1985; Schulz-Hardt \& Frey, 1999; Thaler, 1980). Garland (1990) nennt dieses Verhalten „gutes Geld dem schlechtem hinterherwerfen“. Auch Projekte der öffentlichen Hand überschreiten häufig ihre vorgegebenen Budgets und können zu einer regelrechten Kostenexplosion führen (vgl. Ross \& Staw, 1993). Denkt man in Deutschland an budgetüberschreitende Großprojekte, wie den Bau des Flughafens Berlin Brandenburg oder den Stuttgarter Bahnhof 21, ließe sich aus psychologischer Sicht auch hierbei vermuten, dass ihre kostenintensive Fortsetzung, neben anderen Einflussfaktoren, in Teilen auf den SunkCost-Effekt zurückgeführt werden kann.

Obwohl versunkene Kosten für viele Entscheidungsträger ${ }^{3}$ schwer zu ignorieren sind, finden sich in der Literatur Hinweise, dass die Persönlichkeitsvariablen Handlungs- und Lageorientierung (van Putten, Zeelenberg \& van Dijk, 2010), Optimismus und Pessimismus (Juliusson, 2006), der regulatorische Fokus: Promotion und Prevention (Molden \& Hui, 2011) sowie der Rational Thinking Style (Wong, Kwong \& Ng, 2008) darüber mitbestimmen

\footnotetext{
2 Anders verhielte es sich bspw. bei einer Evaluation der Vorteilhaftigkeit eines Projekts in einer abgeschlossenen Periode in Form einer Gesamtkostenrechnung (vgl. Hummel \& Männel, 1986). Diese Betrachtung ist jedoch nicht mit der vorliegenden, zukunftsbezogenen Entscheidungssituation zu verwechseln.

${ }^{3}$ Im Sinne einer besseren Lesbarkeit wurde in der vorliegenden Arbeit auf eine geschlechtsneutrale Differenzierung (z.B. Entscheidungsträger / Entscheidungsträgerin) verzichtet und entweder die männliche oder weibliche Form von personenbezogenen Hauptwörtern gewählt. Dieses impliziert keinerlei Benachteiligung des jeweils anderen Geschlechts, denn Frauen und Männer sind dabei gleichermaßen angesprochen.
} 
können, ob und in welchem Umfang Personen anfällig für den Sunk-Cost-Effekt sind. Durch eine Berücksichtigung der zugrundeliegenden Persönlichkeitseffekte könnten sich in der Praxis daher Möglichkeiten ergeben, die Auftretenswahrscheinlichkeit für Sunk-Cost-Effekte $\mathrm{zu}$ reduzieren. Grundlegend wäre jedoch, dass die entsprechenden Originalbefunde auch belastbar sind. Das Ziel der vorliegenden Arbeit ist daher - insbesondere vor dem Hintergrund der gegenwärtigen Replikationskrise in der Forschung - jene Befunde auf ihre Reliabilität zu überprüfen und gegebenenfalls $\mathrm{zu}$ erweitern, sodass im Anschluss klar ist, welche Persönlichkeitseffekte wirklich für eine Berücksichtigung in der Praxis empfohlen werden können. 


\section{Theoretischer und empirischer Hintergrund}

Das folgende Kapitel liefert einen Überblick über die theoretischen und empirischen Hintergründe der vorliegenden Arbeit. Dabei werden im ersten Unterkapitel allgemeine Grundlagen des Sunk-Cost-Effekts behandelt. Im zweiten Unterkapitel werden Variablen vorgestellt, für die empirische Arbeiten nahelegen, dass sie die Auftretenswahrscheinlichkeit des Sunk-Cost-Effekts moderieren können. Da sich hieraus wertvolle praktische Implikationen ergeben könnten, stellt die Untersuchung dieser Variablen den zentralen Ansatz der vorliegenden Arbeit dar. Das letzte Unterkapitel geht auf die gegenwärtige Replikationskrise in der Forschung ein, aus der sich die Notwendigkeit zur Durchführung von Replikationsstudien im Rahmen dieser Arbeit ergibt. 


\subsection{Der Sunk-Cost-Effekt}

Dieses Unterkapitel stellt im ersten Abschnitt die verschiedenen Erscheinungsformen des Sunk-Cost-Effekts und seinen zentralen Erklärungsansatz vor. Im zweiten Abschnitt wird auf die Empirie des Sunk-Cost-Effekts eingegangen und eine Abgrenzung zu themenverwandten Forschungsfeldern vorgenommen. Im letzten Abschnitt werden die verschiedenen Operationalisierungsmöglichkeiten in Sunk-Cost-Untersuchungen sowie die in dieser Arbeit verwendeten Sunk-Cost-Szenarien vorgestellt.

\subsubsection{Formen des Sunk-Cost Effekts}

Hal R. Arkes und Catherine Blumer, welche wichtige Pionierarbeit in der Sunk-CostForschung geleistet haben, definieren den Sunk-Cost-Effekt wie folgt: „, The sunk cost effect is manifested in a greater tendency to continue an endeavor once an investment in money, effort, or time $e^{4}$ has been made." (Arkes \& Blumer, 1985, S. 124). Eine alternative Beschreibung des Sunk-Cost-Effekts stammt von Thaler (1980): ,[...] paying for the right to use a good or service will increase the rate at which the good will be utilized, ceteris paribus. " (Thaler, 1980, S. 47).

Beide Definitionen beschreiben den Sunk-Cost-Effekt, allerdings unterscheiden sie sich in Bezug auf die Entscheidung, die auf Basis der versunkenen Kosten getroffen wird: Während Arkes und Blumer (1985) die Fortsetzung eines Unterfangens beschreiben, spricht Thaler (1980) von einer gesteigerten Nutzungsrate. In diesem Sinne lassen sich grundsätzlich zwei verschiedene Erscheinungsformen des Sunk-Cost-Effekts voneinander abgrenzen, nämlich zum einen der Sunk-Cost-Effekt infolge einer Fortführungsentscheidung und zum anderen der

\footnotetext{
${ }^{4}$ Hinsichtlich versunkener Zeitkosten ist in der Literatur umstritten, ob diese Ressource für die Entstehung des Sunk-Cost-Effekts genauso zu bewerten ist, wie monetäre Kosten. Unterstützende Ergebnisse finden sich z.B. bei Greitemeyer, Schulz-Hardt, Popien und Frey (2005) oder Cunha und Caldieraro (2009). Letztere konnten von Otto (2010) hingegen nicht repliziert werden. Auch Coleman (2010) und Soman (2001) konnten in ihren Studien keinen Sunk-Cost-Effekt für zeitliche versunkene Kosten nachweisen. Die vorliegende Arbeit konzentriert sich ausschließlich auf die Untersuchung des Sunk-Cost-Effekts durch monetäre versunkene Kosten, da dieser empirisch besser belegt ist.
} 
Sunk-Cost-Effekt infolge einer Nutzungsentscheidung (vgl. Moon, 2001; Robbert, Roth \& Straus, 2013).

Ein Beispiel für den Sunk-Cost-Effekt in Bezug auf Nutzungsentscheidungen ist die eingangs erwähnte Feldstudie, in der mehr Pizzastücke von einem Buffet konsumiert wurden, wenn für den Zugang zum Buffet der reguläre Preis, im Vergleich zu einem rabattierten Preis, bezahlt wurde (Just \& Wansink, 2011). Fortführungsentscheidungen beinhalten hingegen eine erneute Investitions- bzw. Reallokationsentscheidung. Diese determiniert den Abbruch, die Fortführung oder sogar die Intensivierung eines laufenden Projekts, für das bereits Ausgaben getätigt wurden (vgl. Schulz-Hardt, Vogelgesang \& Mojzisch, 2007). Hierbei sind auch spezifische Zeitpunkte für den Start und das Ende des jeweiligen Entscheidungsgegenstands festgelegt (Moon, 2001). Da in allen Originalstudien der in dieser Arbeit zu untersuchenden Persönlichkeitseffekte der Sunk-Cost-Effekt anhand von Investitionsentscheidungen bestimmt wurde, stehen diese auch im Fokus der vorliegenden Arbeit.

Fortführungsentscheidungen sind allerdings keine universelle Reaktion auf versunkene Kosten. Der Sunk-Cost-Effekt tritt z.B. auch auf, wenn objektiv vorteilhafte Projekte aufgrund versunkener Kosten vorzeitig abgebrochen werden. Z.B. konnte Heath (1995) in seinen Studien zeigen, dass Personen sich selbst dann von versunkenen Kosten fehlleiten lassen und in der Folge profitable Projekte abbrechen, wenn man ihnen eindeutige Zahlen über die zukünftige Vorteilhaftigkeit einer Investition vorlegt. Diese Form des Sunk-CostEffekts findet man in der Literatur auch unter dem Begriff „Reverse-Sunk-Cost-Effekt“ (Johnstone, 2002; Zeelenberg \& van Dijk, 1997).

Den zentralen Erklärungsansatz für das Auftreten des Sunk-Cost-Effekts stellt nach gegenwärtigem Forschungsstand die Verschwendungs-Vermeidungs-Hypothese von Arkes und Blumer (1985) dar. Demnach sind versunkene Kosten nicht einfach zu ignorieren, weil ein Entscheidungsträger nicht verschwenderisch erscheinen möchte und die Abkehr von einem einmal eingeschlagenen Kurs wie eine Verschwendung der hierfür bereits investierten 
Ressourcen wirken würde. Dass man nichts verschwenden sollte ist eine Regel, die die meisten Menschen bereits in ihrer Kindheit lernen, jedoch stellt ihre Anwendung in SunkCost-Situationen eine unangemessene Verallgemeinerung ebendieser Regel dar und führt zur Entstehung des Sunk-Cost-Effekts (vgl. Arkes \& Ayton, 1999; Larrick, Morgan \& Nisbett, 1990). Neben der Verschwendungs-Vermeidungs-Hypothese gibt es auch weitere Erklärungsansätze, wie z.B. Mental Accounting (Thaler, 1985, 1999) oder die ProspectTheory (Kahneman \& Tversky, 1979), die aber nicht alle Formen des Sunk-Cost-Effekts erklären können. ${ }^{5}$

\subsubsection{Empirie und Abgrenzung zu themenverwandten Konstrukten}

Der Sunk-Cost-Effekt wurde bereits in zahlreichen empirischen Studien belegt (vgl. Sleesman, Conlon, McNamara \& Miles, 2012). Nachweise finden sich sowohl für absolute versunkene Kosten als auch für relative versunkene Kosten (vgl. Garland \& Newport, 1991). Es hat sich außerdem gezeigt, dass nicht nur Individuen dem Effekt unterliegen, sondern auch Gruppen (vgl. Smith, Tindale \& Steiner, 1998). Dass es sich bei dem Sunk-Cost-Effekt keinesfalls nur um einen Urteilsfehler ungeschulter Entscheidungsträger handelt, zeigten zum Beispiel Greitemeyer, Schulz-Hardt, Popien und Frey (2005), die in ihrer Studie den SunkCost-Effekt auch für Bankangestellte nachweisen konnten, obwohl diese Personen aufgrund ihres Berufes eigentlich mit der Theorie über versunkene Kosten vertraut sein dürften und

\footnotetext{
${ }^{5}$ Die Prospect-Theory kann bspw. nur den Subtyp der irrationalen Reinvestition erklären. Arkes und Blumer (1985) merken außerdem an, dass die Theorie nicht die psychologische Grundlage erklärt, warum sichere Verluste als aversiv empfunden werden und versunkene Kosten schwer zu ignorieren sind. Gemäß des Mental Accountings verbuchen Menschen thematisch zusammenhängende Geldbewegungen auf mentalen Konten und investieren nicht, sofern hierdurch eine gesetzte Budgetobergrenze überschritten würde oder negative Salden entstünden (vgl. Heath, 1995).
} 
somit einen gewissen Expertenstatus innehaben. Dies unterstreicht wiederum die praktische Relevanz des Sunk-Cost-Effekts. ${ }^{6}$

Unglücklicherweise kommt es in der Literatur zu Reallokationsentscheidungen oft zu einer Vermischung mit den themenverwandten Konstrukten Entrapment (Brockner \& Rubin, 1985) und Escalation of Commitment (Staw, 1976, 1981, 1997). Gemeinsam mit dem Sunk-CostEffekt (Arkes \& Blumer, 1985) bilden sie drei Zweige zur Erforschung psychologisch motivierter Verlusteskalation, die sich mit der Frage beschäftigen, „,warum und unter welchen Bedingungen Menschen irrational an fehlgehenden Handlungen festhalten oder diese sogar intensivieren“ (Schulz-Hardt \& Frey, 1999, S. 487). Forschungsbereichsübergreifend wird dieses Verhalten als „Eskalation“ bezeichnet. Da versunkene Kosten in allen drei Forschungszweigen grundlegend sind, sprechen Greitemeyer et al. (2005) hinsichtlich Entrapment und Escalation of Commitment sogar von „Spezialfällen des allgemeinen SunkCost-Effekts“ (Greitemeyer et al., 2005, S. 36).

Der entscheidende Unterschied zwischen den Konstrukten ergibt sich jedoch aus ihrer Messung. Für Entrapment wird die Verweildauer innerhalb entsprechender Szenarien gemessen. Das Ausmaß von Escalation of Commitment bemisst sich anhand der Summe der Reallokationsmittel, die in die ursprünglich gewählte Alternative reinvestiert wird, während die Finanzmittel frei auf verschiedene Alternativen verteilbar sind. Auf Grundlage der Definitionen von Thaler (1980) und Arkes und Blumer (1985) sowie den grundlegenden Experimenten letzterer, ist das Verständnis der vorliegenden Arbeit, dass für die Messung des reinen Sunk-Cost-Effekts lediglich entscheidend ist, ob Personen überhaupt einen

\footnotetext{
${ }^{6}$ Es sei an dieser Stelle angemerkt, dass eine Reihe von Arbeiten sich außerdem mit der Frage beschäftigt, ob der Sunk-Cost-Effekt auch für Tiere nachweisbar ist. In den entsprechenden Untersuchungen bleibt häufig jedoch unklar, welche Mechanismen dem Verhalten der Versuchstiere zugrunde liegen und ob es sich bei den entsprechenden Befunden tatsächlich um Nachweise für Sunk-Cost-Effekte handelt (siehe z.B. Pattison, Zentall \& Watanabe, 2012). Arkes und Ayton (1999) positionieren sich klar gegen die Annahme, Tiere könnten einen Sunk-Cost-Effekt aufweisen, da ihrem Verhalten keine Verallgemeinerung abstrakter Regeln wie der Verschwendungs-Vermeidungs-Heuristik zugrunde liegt. Im Rahmen der vorliegenden Arbeit, die den Einfluss menschlicher Persönlichkeitsvariablen auf den Sunk-Cost-Effekt untersucht, ist eine weitere Behandlung dieser Fragestellung nicht relevant.
} 
eingeschlagenen Kurs aufgrund versunkener Kosten fortsetzen oder nicht. Diese Entscheidung bemisst sich unabhängig von der investierten Verweildauer oder Reallokationssumme.

\subsubsection{Operationalisierung in Sunk-Cost-Untersuchungen}

In Sunk-Cost-Untersuchungen werden den Probanden in der Regel hypothetische, in sich abgeschlossene Fall-Vignetten vorgelegt, in denen ein Szenario beschrieben wird, an das sich die Frage nach einer Fortführungs- bzw. Nutzungsentscheidung anschließt (vgl. Schulz-Hardt \& Frey, 1999). Essentiell ist dabei die Nennung, ob (bzw. für welche der aufgeführten Alternativen) bereits versunkene Kosten in Form von Zeit, Geld oder sonstigen Anstrengungen entstanden sind. Eine Incentivierung findet im Rahmen der Versuchspersonenvergütung typischerweise nicht statt. Wie erwähnt ist für die Bestimmung des Sunk-Cost-Effekts entscheidend, ob sich die Probanden für oder gegen die mit versunkenen Kosten behafteten Alternative entscheiden. Grundsätzlich lässt sich der SunkCost-Effekt auf diesem Wege durch zwei verschiedene Strategien nachweisen (vgl. Arkes \& Blumer, 1985):

Die erste Strategie ist die Messung des Sunk-Cost-Effekts auf Individualebene, wie z.B. in Arkes und Blumers Ski-Trip- oder Dinner-Szenario (1985, Experiment 1 \& 6). In diesem Fall werden der Versuchsperson gleichzeitig verschiedene Wahlalternativen vorgelegt, von denen eine höhere versunkene Kosten aufweist als die anderen Alternativen. Sind die individuellen Präferenzen wie im Ski-Trip-Szenario mit angegeben, sollte sich ein rationaler Entscheider unabhängig von den versunkenen Kosten - für die Alternative mit dem besten zukünftigen Kosten-Nutzen-Verhältnis entscheiden. Für den Fall, dass die zukünftigen Kosten und der zukünftige Nutzen der Alternativen wie im Dinner-Szenario identisch sind, sollte sich der Entscheider indifferent verhalten. Der Sunk-Cost-Effekt zeigt sich bei dieser Strategie für jede 
einzelne Person, sofern sie ungeachtet des Kosten-Nutzen-Verhältnisses die Alternative mit (hohen) versunkenen Kosten wählt.

Die zweite Strategie ist ein Vergleich von experimentellen Bedingungen, indem die versunkenen Kosten inter-individuell variiert werden. Hierfür werden bspw. zwei Faktorstufen für die versunkenen Kosten in das Versuchsdesign integriert, wobei auf einer Faktorstufe versunkene Kosten vorliegen $\left(\mathrm{SC}^{(+)}\right)$, während auf der anderen Stufe, zum Zwecke einer Baseline-Messung, keine oder geringere versunkene Kosten vorliegen $\left(\mathrm{SC}^{(-)}\right)$. Die Probanden werden zu Beginn randomisiert auf die Versuchsbedingungen beider Faktorstufen aufgeteilt. Anschließend wird ihnen z.B. ein Investitionsszenario zur Bearbeitung vorgelegt, dass sich zwischen den Bedingungen inhaltlich nur in Bezug auf die versunkenen Kosten unterscheidet. Der Sunk-Cost-Effekt kann in diesem Fall durch einen aggregierten Gruppenvergleich nachgewiesen werden, sofern sich zwischen den Versuchsbedingungen Investitionsunterschiede feststellen lassen, obwohl unter Abwägung des zukünftigen Kosten-Nutzen-Verhältnisses normativ kein Unterschied bestehen sollte (vgl. z.B. Arkes \& Blumer, 1985, Experiment 3 \& 7).

In der Sunk-Cost-Forschung wird gelegentlich auf die Integration einer $\mathrm{SC}^{(-)}$-Faktorstufe in das Versuchsdesign verzichtet und in den eingesetzten Szenarien stattdessen allein mit vorhandenen versunkenen Kosten operiert. Dieses erfolgt vor dem Hintergrund, dass bei der Versuchsplanung der Standpunkt vertreten wird, dass in den Szenarien hinreichend deutlich werde, dass eine Fortführung unter den gegebenen Umständen nicht sinnvoll sei. In diesen Fällen bleibt jedoch unklar, ob z.B. eine mehrheitliche Investitionsneigung spezifisch auf den Sunk-Cost-Effekt oder auf eine unspezifisch erhöhte Investitionsfreudigkeit zurückzuführen ist. Die Variation der versunkenen Kosten durch die Integration einer SC ${ }^{(-)}$-Faktorstufe in das Versuchsdesign ist daher ein wesentlicher Bestandteil zur Bestimmung des Sunk-Cost-Effekts durch einen Gruppenvergleich. 
In der Arbeit von Arkes und Blumer (1985) findet sich eine Reihe von Sunk-CostSzenarien, die seither regelmäßig in der Sunk-Cost-Forschung verwendet werden und sich empirisch gut bewährt haben. Zwei dieser Szenarien werden auch in den späteren Untersuchungen der vorliegenden Arbeit eingesetzt und sollen daher in den folgenden zwei Abschnitten kurz vorgestellt werden. Bei dem ersten Szenario handelt es sich um das „Airplane-Szenario“ von Arkes und Blumer (1985, Experiment 3), welches auch in drei der vier Originalarbeiten über Persönlichkeitseffekte zum Einsatz kam, die im Rahmen der vorliegenden Arbeit überprüft werden sollen. Bei dem zweiten Szenario handelt es sich um das „Druckerpressen-Szenario“ von Arkes und Blumer (1985, Experiment 7). Für beide Investitionsszenarien existiert je eine $\mathrm{SC}^{(+)}$- und eine $\mathrm{SC}^{(-)}$-Version, um den Sunk-Cost-Effekt durch einen Vergleich von experimentellen Bedingungen bestimmen zu können. Die beiden Szenarien finden sich in deutscher Übersetzung in Anhang A.

\subsubsection{Airplane-Szenario}

Im Airplane-Szenario von Arkes und Blumer (1985, Experiment 3) werden die Teilnehmer gebeten sich vorzustellen, sie seien der Präsident einer Fluggesellschaft und müssten eine Entscheidung über die Investition von einer Million Dollar in ein Forschungsprojekt treffen. In dem Projekt wird ein Flugzeug entwickelt, welches von einem herkömmlichen Radar nicht erfasst werden kann. Zusätzlich wird darauf hingewiesen, dass eine andere Firma bereits begonnen habe, ein Konkurrenzprodukt zu vermarkten, welches wesentlich schneller und sehr viel ökonomischer sei als das eigene Flugzeug.

Der zentrale Unterschied zwischen den Szenario-Versionen ist, dass das Projekt in der $\mathrm{SC}^{(+)}$-Version zu 90\% fertiggestellt ist und bereits neun von zehn Millionen Dollar investiert wurden. In der $\mathrm{SC}^{(-)}$-Version handelt es sich um eine noch nicht gestartete Projektidee ohne versunkene Kosten. Mit der Investition in Höhe von einer Millionen Dollar könnte das Projekt 
in beiden Versionen jeweils finalisiert werden, eine Nicht-Investition führt zu einem Abbruch des Projekts.

In Arkes und Blumers (1985) Studie zeigte sich ein signifikanter Sunk-Cost-Effekt in der Form, dass sich $85 \%$ (41 von 48) der Probanden in der $\mathrm{SC}^{(+)}$-Bedingung für eine Investition entschieden, während es in der SC ${ }^{(-)}$-Bedingung nur 17\% (10 von 60) waren.

\subsubsection{Druckerpressen-Szenario}

Im Druckerpressen-Szenario von Arkes und Blumer (1985, Experiment 7) werden die Teilnehmer gebeten sich vorzustellen, sie seien Eigentümer einer Druckerei, welche sie in der Vergangenheit gerne im Wert von 200.000 Dollar modernisieren wollten. Aus diesem Grund haben sie bereits entweder eine neue Druckerpresse in der $\mathrm{SC}^{(+)}$-Version oder neue Transporter in der $\mathrm{SC}^{(-)}$-Version gekauft. Anschließend wird ihnen eine gebrauchte Druckerpresse von einem bankrotten Wettbewerber für 10.000 Dollar kurzfristig zum Kauf angeboten. Diese produziere deutlich schneller und kostengünstiger als die eigene Druckerpresse, d.h., je nach Version, entweder die gerade gekaufte oder die bisher im Betrieb befindliche. Der Kauf der gebrauchten Druckerpresse könnte aus den Rücklagen des Unternehmens finanziert werden.

Auch in dieser Studie zeigte sich ein signifikanter Sunk-Cost-Effekt bei Arkes und Blumer (1985), jedoch in Form einer Nicht-Investition. In der SC ${ }^{(+)}$-Bedingung entschieden sich $47 \%$ (38 von 81) der Probanden gegen den Kauf der gebrauchten Druckerpresse, während es in der $\mathrm{SC}^{(-)}$-Bedingung nur 23\% (15 aus 64) waren. 


\subsection{Moderatorvariablen des Sunk-Cost-Effekts}

Das folgende Unterkapitel gibt einen Überblick über in der Literatur berichtete Moderatorvariablen des Sunk-Cost-Effekts. Diese sind allein zwar nicht ursächlich für den Sunk-Cost-Effekt, können seine Auftretenswahrscheinlichkeit aber entscheidend mit beeinflussen. Inhaltlich lassen sich dabei zwei größere Gruppen von Moderatorvariablen unterscheiden, nämlich erstens situative Variablen des jeweiligen Entscheidungskontextes und zweitens Persönlichkeitsvariablen des Entscheidungsträgers. Da der Schwerpunkt der vorliegenden Arbeit auf den Persönlichkeitsvariablen liegt, sollen im folgenden Abschnitt nur einige Beispiele häufig zitierter situativer Moderatorvariablen des Sunk-Cost-Effekts genannt werden. Anschließend erfolgt eine ausführliche Darstellung aller Persönlichkeitsvariablen, die im Rahmen der vorliegenden Arbeit überprüft werden sollen.

\subsubsection{Situative Moderatorvariablen}

In Sunk-Cost-Situationen spielen die finanziellen Rahmenbedingungen bei der Entscheidungsfindung eine gewichtige Rolle. Garland (1990) variierte zum Beispiel die Höhe der versunkenen Kosten und berichtete, dass die Bereitschaft seiner Probanden, weitere Ressourcen zu investieren, positiv und linear mit der Höhe der versunkenen Kosten anstieg. Wie sich Entscheidungsträger verhalten, deren Investitionsbudget überraschend vergrößert wird, untersuchten Soman und Cheema (2001). Den Autoren folgend kann ein unerwarteter Gewinn im Zeitpunkt der Entscheidungsfindung die Sunk-Cost-Neigung reduzieren. Soman und Gourville (2001) berichteten eine Abschwächung des Sunk-Cost-Effekts durch Preisbündelung. Personen, die für ihren viertägigen Skiurlaub einen gebündelten Skipass gekauft hatten, waren eher bereit auf das Skifahren am letzten Urlaubstag zu verzichten, als Personen, die vier separate Tagestickets zum gleichen Gesamtpreis wie im Bündel gekauft hatten. Den gleichen Effekt fanden die Autoren auch auf Basis realer Verkaufsdaten von 
Tickets eines Theaterfestivals, die ebenfalls entweder gebündelt für mehrere Vorstellungen oder als Einzeltickets verkauft wurden.

Neben den finanziellen Rahmenbedingungen lassen sich weitere situative Variablen in der Literatur identifizieren. Zum Beispiel berichteten Hafenbrack et al. (2014), dass eine fünfzehnminütige Meditationsphase, durch die der Aufmerksamkeitsfokus des Entscheidungsträgers auf den derzeitigen Moment gelenkt wird, zu einer Abschwächung der Sunk-Cost-Neigung führen kann. Gourville und Soman (1998) berichteten, dass versunkene Kosten nach einer gewissen zeitlichen Dauer diskontiert werden, wodurch die Sunk-CostNeigung im Zeitverlauf abnimmt. Tan und Yates (1995) folgend, reduziert auch die Kenntnis ökonomischer Prinzipien die Sunk-Cost-Neigung, sofern dieses Wissen in enger Verbindung zum Entscheidungsproblem steht (siehe auch Greitemeyer et al., 2005).

\subsubsection{Persönlichkeitsvariablen}

Den Schwerpunkt der vorliegenden Arbeit bildet eine Überprüfung von Persönlichkeitsvariablen, für die eine Reihe empirischer Arbeiten nahelegt, dass diese darüber mitentscheiden können, ob und in welchem Umfang Entscheidungsträger anfällig für den Sunk-Cost-Effekt sind. Hierbei handelt es sich um insgesamt vier Variablen, nämlich Handlungs- und Lageorientierung (van Putten et al., 2010), Optimismus und Pessimismus (Juliusson, 2006), den regulatorischen Fokus: Promotion und Prevention (Molden \& Hui, 2011) sowie den Rational Thinking Style (Wong et al., 2008). Vor dem Hintergrund, dass Autoren gelegentlich den Sunk-Cost-Effekt mit Escalation of Commitment gleichsetzen (vgl. Abschnitt 2.1.2), wurde bei der Auswahl dieser Variablen primär darauf geachtet, ob in den zugrundeliegenden Originalstudien typisches Sunk-Cost-Material verwendet wurde, wie in Abschnitt 2.1.3 beschrieben.

Da der Sunk-Cost-Effekt vor allem in ökonomischer Hinsicht mit zum Teil erheblichen negativen Konsequenzen verbunden sein kann, könnte sich eine Berücksichtigung der in den 
Originalarbeiten berichteten Effekte dieser Persönlichkeitsvariablen in der Praxis positiv auswirken. Schließlich hängt der wirtschaftliche Erfolg eines Unternehmens stark von der Qualität seiner Investitionsentscheidungen ab, da diese eine längerfristige Bindung und damit längerfristige Erfolgswirkungen für das Unternehmen schaffen (vgl. Dietrich, 1993). Um den Unternehmenserfolg nicht durch irrationale Investitionsentscheidungen, die auf Sunk-CostEffekte zurückzuführen sind, zu gefährden, könnte ein Unternehmen beispielsweise durch eine geeignete Personalauswahl und Personaldiagnostik wichtige Entscheidungspositionen vorrangig mit Entscheidungsträgern besetzen, die aufgrund ihrer Persönlichkeit nicht oder nur wenig anfällig für den Sunk-Cost-Effekt sind. Denkbar wäre auch, Entscheidungsträger z.B. durch Trainings in bestimmte temporäre Persönlichkeitszustände zu versetzen, die ihre SunkCost-Neigung reduzieren.

Es ist eine grundlegende psychologische Annahme aus der Eigenschafts- und Verhaltensdiagnostik, dass Persönlichkeitseigenschaften zur Beschreibung menschlichen Verhaltens herangezogen werden können (vgl. Schmidt-Atzert \& Amelang 2012). ${ }^{7}$ Hinsichtlich der psychologischen Persönlichkeitsvariablen wird dabei zwischen chronischen Persönlichkeitseigenschaften (Traits) und situativen Zuständen (States) differenziert. Traits sind relativ breite und zeitlich stabile Dispositionen des Verhaltens. Sie treten zustands- und situationsunabhängig auf und stellen somit ein globales Abbild eines Teils der Persönlichkeit dar. States hingegen sind fluktuierende, temporäre Zustände, die von der jeweiligen Situation abhängen (vgl. Moosbrugger \& Kelava, 2012; Schmidt-Atzert \& Amelang 2012). Sowohl Traits als auch States beeinflussen gleichsam das menschliche Verhalten und werden daher im Rahmen dieser Arbeit als gleichwertig relevant betrachtet und untersucht.

\footnotetext{
${ }^{7}$ Nicht-psychologische Personenmerkmale, wie das Alter, wurden in der vorliegenden Arbeit nicht untersucht. Im Falle des Alters handelt es sich um eine demographische Variable, bei der noch weitgehend ungeklärt ist, welche psychologischen Prozesse den jeweiligen Effekt vermitteln (vgl. Arkes \& Ayton, 1999; Bruine de Bruin, Parker \& Fischhoff, 2007; Klaczynski \& Cottrell, 2004; Strough, Schlosnagle, Karns, Lemaster \& Pichayayothin, 2014; Strough, Mehta, McFall \& Schuller, 2008).
} 
In den vier folgenden Abschnitten werden die Originalarbeiten der genannten Persönlichkeitsvariablen nacheinander vorgestellt. Bei den Studien zu Handlungs- und Lageorientierung sowie dem Rational Thinking Style handelte es sich jeweils um Untersuchungen auf Trait-Ebene; Optimismus und Pessimismus sowie der regulatorische Fokus wurden jeweils auf State-Ebene experimentell induziert.

\subsubsection{Handlungsorientierung und Lageorientierung}

Van Putten et al. (2010) berichteten einen moderierenden Einfluss von Trait-Handlungsund Lageorientierung (siehe Kuhl \& Beckmann, 1994a) auf den Sunk-Cost-Effekt und zwar in dem Sinne, dass handlungsorientierte Personen weniger anfällig für den Sunk-Cost-Effekt seien als lageorientierte Personen.

Das Persönlichkeitskonstrukt der Handlungs- und Lageorientierung steht in einer Reihe mit artverwandten Konstrukten wie Gewissenhaftigkeit, Neurotizismus, Angst oder Intro- vs. Extraversion (vgl. Kuhl \& Beckmann, 1994b). Es kann als Brückenschlag zwischen einer Handlungsauswahl und dem konkreten Handlungsvollzug verstanden werden, in dem psychologische Faktoren wie Überzeugungen und Wünsche einen verhaltensrelevanten Einfluss gewinnen können. Ihre Verarbeitung erfolgt in Form selbstregulierter psychologischer Mechanismen, die den Anstoß und Beschluss einer angestrebten Handlung herbeiführen. Im Fokus des Konstrukts der Handlungs- und Lageorientierung steht also die psychologische Kompetenz eines Individuums, seinen eigenen Willen umzusetzen. Hiermit lässt sich erklären, warum objektiv rationale Handlungen, wie z.B. absolut vorteilhafte Investments, unter Umständen nicht durchgeführt werden (vgl. Kuhl, 1994).

Die Dimension Lageorientierung zeichnet sich in der Theorie durch ihre hemmenden Effekte auf die Willensfähigkeit eines Individuums aus, angestrebte Maßnahmen zu planen, initiieren und fertigzustellen. Zurückzuführen ist dies auf ein Zögern oder eine Voreingenommenheit des Betroffenen, weil sich wiederholende Kognitionen über ein 
negatives Erlebnis und seinen Einfluss auf die derzeitige Lage fortwährend aufdrängen. Dadurch werden wichtige handlungsleitende Funktionen blockiert. Nach Interpretation von van Putten et al. (2010) kann auch eine mangelnde Kompensation versunkener Kosten als eine solche negative Lage empfunden werden. Ihre Argumentation lautet: je lageorientierter Personen sind, desto mehr betrachten sie gegenwärtige Entscheidungen vor dem Hintergrund vorheriger Investitionen und setzen die beiden Situationen stärker miteinander in Verbindung.

Die Dimension Handlungsorientierung wird in der Theorie hingegen als Fähigkeit beschrieben, kontextabhängige Vorsätze leichter zu beschließen. Handlungsorientierte Personen kommen im Vergleich zu lageorientierten Personen schneller über negative Ereignisse hinweg und konzentrieren sich auf entsprechende Lösungsansätze. Die Argumentation von van Putten et al. (2010) lautet daher: je handlungsorientierter Personen sind, desto mehr legen sie ihren Fokus auf die Zukunft sowie erreichbare Ziele. Dadurch setzen sie aktuelle Entscheidungen und bereits getätigte Investitionen zunehmend weniger miteinander in Verbindung. Indirekte Unterstützung hierfür leiten die Autoren aus einer eigenen vorangegangenen Studie ab, in der stärker handlungsorientierte Personen verpasste Gelegenheiten der Vergangenheit von zugehörigen aktuellen Entscheidungen entkoppelten (van Putten, Zeelenberg \& van Dijk, 2009).

Basierend auf diesen Annahmen stellten van Putten et al. (2010) in Bezug auf den SunkCost-Effekt die Hypothese auf, dass lageorientierte Personen mehr von vorherigen Investitionen beeinflusst werden als handlungsorientierten Personen, wenn sie eine aktuelle Investitionsentscheidung treffen sollen, und daher mit höherer Wahrscheinlichkeit den SunkCost-Effekt aufweisen. Um dieses zu überprüfen, ließen sie ihre Probanden zunächst eine niederländische Übersetzung der Action Control Scale (ACS-90) mit 24 Items bearbeiten (vgl. Kuhl \& Beckmann, 1994a) und maßen damit Handlungs- und Lageorientierung auf Trait-Ebene. Daraus resultierte eine numerische Skala, bei der positive Werte eine stärkere Handlungsorientierung und negative Werte eine stärkere Lageorientierung repräsentierten. 
Eine Woche nach dieser ersten Erhebung kehrten die Probanden ins Labor zurück, um das Airplane-Szenario (Arkes \& Blumer, 1985; vgl. Abschnitt 2.1.3.1) zu bearbeiten. Als Kriteriumsvariable wurde dabei die Investitionsentscheidung (investieren: ja vs. nein) erhoben.

Die Ergebnisse bestätigten die zugrundeliegende Hypothese. Neben einem signifikanten Haupteffekt für die versunkenen Kosten zeigte sich auch ihre Interaktion mit dem Persönlichkeitskonstrukt signifikant. Separate Simple-Slope-Analysen ergaben, dass ein Sunk-Cost-Effekt nur für Lageorientierung signifikant nachweisbar war, jedoch nicht für Handlungsorientierung. Eine weitere Analyse erfolgte nach Teilung des Datensatzes durch einen Mediansplit. Danach entschieden sich auf Stufe vorhandener versunkener Kosten signifikant mehr lageorientierte Probanden für eine Investition als auf der kontrastierenden Stufe ohne versunkene Kosten (80\% vs. 20\%). Für handlungsorientierte Personen zeigte sich dieser Unterschied nur deskriptiv und in einem schwächeren Umfang (Entscheidung für die Investition: $53 \%\left[\mathrm{SC}^{(+)}\right]$vs. $\left.33 \%\left[\mathrm{SC}^{(-)}\right]\right)$. Im Vergleich waren handlungsorientierte Personen auf Stufe vorhandener versunkener Kosten zwar weniger investitionsfreudig als lageorientierte Personen, jedoch zeigte sich dieser Unterschied nur marginal. Auf der Stufe ohne versunkene Kosten gab es keinen Unterschied.

Van Puttens et al. (2010) Studie offenbarte Unterschiede hinsichtlich der Anfälligkeit für den Sunk-Cost-Effekt, abhängig von dem Grad der Handlungs- und Lageorientierung auf Trait-Ebene. Handlungsorientierte Personen trafen ihre Investitionsentscheidung im Vergleich zu lageorientierten Personen weitgehend unabhängig von den versunkenen Kosten, woraus die Autoren folgerten, dass Handlungsorientierung zwar kein ,unzerstörbares Schild“ gegen den Sunk-Cost-Effekt forme, jedoch die Wahrscheinlichkeit, in fehlgehende Projekte zu investieren, klar reduziert (van Putten et al., 2010, S. 35).

Einschränkend sei an dieser Stelle erwähnt, dass der Haupteffekt für die versunkenen Kosten sowie die separaten Simple-Slope-Analysen jeweils negative $B$ - 
Regressionskoeffizienten aufwiesen. Im Gegensatz zu den oben dargestellten Ausführungen von van Putten et al. (2010), die aus ihren Ergebnissen eigentlich eine gesteigerte Investitionsneigung im Zuge steigender versunkener Kosten ableiteten, würde ein negativer $B$-Regressionskoeffizient bedeuten, dass sich die Probanden bei steigenden versunkenen Kosten zunehmend weniger investitionsfreudig zeigten. Dieses stünde außerdem im direkten Gegensatz zu dem grundlegenden Befund von Arkes und Blumer (1985). Sowohl die verwendete Abbildung als auch die Tabelle des Artikels stützen hingegen die inhaltlichen Ausführungen der Autoren, wonach die Investitionsneigung bei vorhandenen versunkenen Kosten höher ausgeprägt war als in Abwesenheit versunkener Kosten. Es liegt daher die Vermutung nahe, dass es sich daher um Vorzeichenfehler der genannten Regressionskoeffizienten handeln könnte. Dieses konnte allerdings nicht abschließend geklärt werden, da eine entsprechende Nachfrage an die Erstautorin leider unbeantwortet blieb.

\subsubsection{Optimismus und Pessimismus}

Juliusson (2006) berichtete einen moderierenden Einfluss von zustandsbezogenem Optimismus und Pessimismus auf den Sunk-Cost-Effekt. In seiner Studie führte Optimismus $\mathrm{zu}$ einem gesteigerten Investitionsverhalten und Pessimismus $\mathrm{zu}$ einem gesteigerten Abbruchverhalten. Hohe versunkene Kosten verstärkten diese Verhaltensweisen jeweils in die entsprechende Richtung. ${ }^{8}$

Grundlegend für Juliussons (2006) Untersuchung waren Befunde vorangegangener Studien von Juliusson, Karlsson und Gärling (2005) sowie Karlsson, Juliusson, Grankvist und Gärling (2002), in denen Typ und Transparenz eines vorgegebenen Entscheidungsziels einen Einfluss auf das Investitionsverhalten zeigten. Probanden, denen Gewinnmaximierung als Ziel vorgegeben wurde, setzten ihre Investitionen häufiger fort als Probanden mit dem Ziel der

\footnotetext{
${ }^{8}$ Wie einleitend erwähnt, spricht Juliusson (2006) nomenklatorisch zwar von Escalation of Commitment, adressiert inhaltlich aber den Sunk-Cost-Effekt (vgl. Abschnitt 2.1.2). Bei einer Betrachtung des eingesetzten Materials wird klar, dass es sich unabhängig davon um eine reine Sunk-Cost-Studie handelte (vgl. Abschnitt 2.1.3).
} 
Verlustminimierung. Ein Zusammenhang zu Optimismus und Pessimismus ergibt sich laut Juliusson (2006) aus den in den Studien verwendeten Instruktionen. Darin wurde den Probanden ein Investitionskontext mit Angaben über zukünftige Erfolgsaussichten vorgelegt. Juliusson (2006) beschreibt, dass aufgrund der Darstellung auf der Faktorstufe mit erfolgversprechenden Zukunftsaussichten der Eindruck habe entstehen können, dass auf gegenwärtige Gewinnerwartungen weitere zukünftige Gewinne folgen würden. Auf der Stufe mit schlechten Zukunftsaussichten könne hingegen der Eindruck fortwährender zukünftiger Verluste entstanden sein. Er vertritt daher die Auffassung, dass hierdurch ein optimistischer bzw. pessimistischer Zustand bei den Probanden induziert wurde, wodurch sich die gefundenen Eskalations- bzw. Deeskalations-Effekte der angesprochenen Vorläuferstudien alternativ erklären ließen. Unter Verweis auf Arkes und Hutzel (2000) erklärt der Autor weiter, dass bereits in vorherigen Studien Optimismus in Form einer erhöhten Gewinnwahrscheinlichkeit nach einer initialen Investition operationalisiert wurde. In den von Juliusson (2006) beispielhaft angeführten Studien von Cooper, Woo und Dunkelberg (1988) sowie Schmidt und Calantone (1998) führte Optimismus außerdem zu einem gesteigerten Eskalationsverhalten. $^{9}$

In seiner eigenen Studie induzierte Juliusson (2006) Optimismus und Pessimismus wie folgt: Die Probanden bearbeiteten Investitionsszenarien und wurden informiert, dass die Gewinnwahrscheinlichkeit des entsprechenden Investitionsprojekts in der Phase der initial

\footnotetext{
${ }^{9}$ Cooper et al. (1988) befragten 2994 Unternehmer, die gerade erst ihre neue Firma gegründet hatten, zur Einschätzung über die Erfolgswahrscheinlichkeit des Unternehmens. Es zeigte sich eine durchweg hohe optimistische Sichtweise der Befragten, von denen $81 \%$ ihre Gewinnchancen mit 7 von 10 Punkten oder besser bewerteten, ein Drittel vergab sogar 10 Punkte $(M=8.1)$. Außerdem erwiesen sich die schlecht vorbereiteten Unternehmer als genauso optimistisch wie die gut vorbereiteten.

Schmidt und Calantone (1998) ließen Manager Investitionsentscheidungen über Airbag-Sensoren für PKW treffen, die entweder ein innovatives Produkt waren oder eine stufenweise Entwicklung herkömmlicher Sensoren. Im Ergebnis wurde eine Fortführungsentscheidung signifikant öfter für die innovativen Sensoren getroffen, selbst bei negativen Finanzinformationen. Gleichzeitig schätzten die Probanden die Erfolgswahrscheinlichkeit für innovative Sensoren höher ein als für die herkömmlichen. Dieser Umstand habe die Probanden enger an das Projekt gebunden und sie mit höherer Wahrscheinlichkeit eine Reinvestition treffen lassen, so die Autoren. Dieses war selbst dann der Fall, als in einer frühen Projektphase negative Zukunftsinformationen einen Projektabbruch eindeutig als rationale Alternative kennzeichneten und die versunkenen Kosten noch nicht so hoch gestiegen waren.
} 
getätigten Investition, je nach Faktorstufe, entweder $80 \%$ (Pessimismus) oder 20\% (Optimismus) betrug. In der zweiten Phase, in der die Probanden gebeten werden das Projekt mit einer Reinvestition fortzusetzen oder abzubrechen, ändert sich für alle Probanden die Gewinnwahrscheinlichkeit auf einen Erwartungswert von 50\%. ${ }^{10}$ Durch einen Vergleich der aktuellen mit der ursprünglichen Gewinnwahrscheinlichkeit werden den Probanden Trends suggeriert und in der Folge Optimismus durch einen positiven Trend und Pessimismus durch einen negativen Trend induziert. Eine separat durchgeführte Studie zur Manipulationskontrolle von Juliusson (2006) bestätigte die Wirksamkeit dieser Manipulation. Juliusson (2006) stellte die Hypothese auf, dass optimistische Probanden die Wahrscheinlichkeit für zukünftige Gewinne inflationieren und darum stärker gewillt seien, ein Investment fortzusetzen als pessimistische Probanden, die im Gegensatz dazu die Wahrscheinlichkeit für zukünftige Gewinne deflationieren. Hinsichtlich des Sunk-CostEffekts erwartete Juliusson (2006) unter Verweis auf Kukla (1974) darüber hinaus, dass der Wunsch optimistischer Probanden, versunkene Kosten wieder auszugleichen, mit höheren versunkenen Kosten stärker zunimmt und somit zu einem Eskalationsverhalten führt. Unter Verweis auf Kahneman und Tversky (1984) ging Juliusson (2006) bei pessimistischen Probanden stattdessen von einer vorherrschenden Verlustaversion aus. Diese würde bei höheren versunkenen Kosten eine geringere Bereitschaft zur Reallokation diktieren, je mehr die Probanden verlieren können, und somit zu einem Deeskalationsverhalten führen.

Seine Hypothesen testete Juliusson (2006) in einem 2 (hohe vs. niedrige versunkene Kosten) $\times 2$ (exakte vs. ungefähre Gewinnwahrscheinlichkeit) $\times 2$ (Optimismus vs. Pessimismus) - Design mithilfe von vier strukturgleichen, jedoch unabhängigen Sunk-Cost-

\footnotetext{
10 Juliusson (2006) variierte zusätzlich die Gewinnwahrscheinlichkeit als zweistufigen Faktor (exakte vs. ungefähre Gewinnwahrscheinlichkeit). In den Bedingungen der ungefähren Gewinnwahrscheinlichkeit betrug diese ,zwischen 30\% und 70\%“ anstatt exakten 50\%, was jedoch ebenso einem Erwartungswert von 50\% entspricht. Die Variation der Gewinnwahrscheinlichkeit wird im Folgenden nur der Vollständigkeit halber behandelt und besitzt keine Relevanz hinsichtlich des Zusammenhangs zwischen Optimismus vs. Pessimismus und dem Sunk-Cost-Effekt.
} 
Szenarien. Darin wurden die beiden erstgenannten Faktoren intra-individuell variiert, während die entscheidende Manipulation von Optimismus und Pessimismus inter-individuell erfolgte. Die erste abhängige Variable, die Investitionsentscheidung, wurde dichotom erhoben (investieren vs. abbrechen). Zusätzlich sollten die Probanden auf einer Skala von 0 bis 10 angeben, wie sehr sie zu der vorherigen Entscheidung stehen. Aus den beiden erhobenen Werten wurde im Anschluss ein Präferenzindex gebildet, der als zweite abhängige Variable in einer alternativen Analyse von Juliusson (2006) diente. ${ }^{11}$

Im Ergebnis zeigte sich nur für die dichotome Investitionsentscheidung ein signifikanter Interaktionseffekt zwischen den versunkenen Kosten und Optimismus vs. Pessimismus. Dabei wiesen die optimistischen Probanden, denen ein steigender Trend der Gewinnwahrscheinlichkeit suggeriert wurde, eine generelle Investitionsneigung auf, die noch stärker ausgeprägt war, wenn hohe versunkene Kosten im Vergleich zu niedrigen versunkenen Kosten vorlagen. Im Gegensatz dazu zeigten die pessimistischen Probanden, denen ein fallender Trend suggeriert wurde, eine generelle Abbruchneigung, die noch stärker ausfiel, wenn hohe gegenüber niedrigen versunkenen Kosten vorlagen. Ein genereller SunkCost-Effekt war nur auf der Stufe von Optimismus nachweisbar.

\subsubsection{Regulatorischer Fokus: Promotion und Prevention}

Molden und Hui (2011) berichteten, dass ein Promotion Focus, im Sinne der Regulatory Focus Theory von Higgins (1997), Personen in die Lage versetze, versunkene Kosten besser ignorieren zu können. ${ }^{12}$

\footnotetext{
${ }^{11}$ Da sich der zentrale Interaktionseffekt zwischen den versunkenen Kosten und Optimismus vs. Pessimismus nur in der Analyse der dichotomen Investitionsentscheidung zeigte, wird an dieser Stelle auf eine detailliertere Beschreibung des Präferenzindex verzichtet und, für nähere Informationen hierüber, auf den später folgenden Abschnitt 4.2.2.2 verwiesen.

${ }^{12}$ Auch Molden und Hui (2011) gebrauchen, wie einleitend beschrieben, den Terminus des eskalierenden Commitments. In ihrer zweiten Studie verwendeten sie hingegen das Airplane-Szenario von Arkes und Blumer (1985), mit dem klassischerweise der Sunk-Cost-Effekt gemessen wird (vgl. Abschnitt 2.1.2 \& 2.1.3) und nicht Escalation of Commitment, wie nach der Meinung der Autoren (vgl. Molden und Hui, 2011, S. 10). Die Schlussfolgerung von Molden und Hui (2011), dass ein Promotion Focus hilfreich sei, um versunkene Kosten besser zu ignorieren, unterstreicht darüber hinaus, dass es sich hier um eine Sunk-Cost-Studie handelte.
} 
Der regulatorische Fokus beschreibt die psychologische Selbstregulierung, die auftritt, wenn ein bestimmter Endzustand (z.B. Freude) angestrebt oder (z.B. Schmerz) vermieden werden soll. Dabei wird zwischen zwei motivationalen Orientierungen unterschieden, nämlich einerseits einer enthusiastischen Orientierung (Promotion Focus) und andererseits einer defensiven Orientierung (Prevention Focus) (vgl. Higgins, 1997, 1998).

Der Promotion Focus impliziert eine Sensitivität für positive Ergebnisse, d.h. die Präsenz von positiven Ergebnissen (z.B. Gewinnen) wird als erfreulich und ihre Abwesenheit als schmerzlich empfunden. Charakteristisch für einen Promotion Focus sind das Bedürfnis nach persönlichem Wachstum und Vollendung, das Streben nach dem Erreichen von Hoffnungen und Wünschen sowie der Antrieb, Gewinne zu erzielen. Im Gegensatz dazu impliziert der Prevention Focus eine Sensitivität für negative Ergebnisse, d.h. die Abwesenheit von negativen Ergebnissen (z.B. Verlusten) wird als erfreulich und ihre Anwesenheit als schmerzlich empfunden. Ein Prevention Focus ist gekennzeichnet durch das Bedürfnis nach Schutz und Sicherheit, dem Wunsch, die eigenen Pflichten und Verantwortlichkeiten zu erfüllen sowie Verluste zu vermeiden (vgl. Idson, Liberman \& Higgins, 2004; Higgins, 1997, 1998; Higgins et al., 2001).

Molden und Hui (2011) beleuchteten die Theorie im Kontext menschlicher Entscheidungsfindung und betonten, dass ein wachstumsorientierter Promotion Focus neben einer Gewinnfokussierung auch mit einer breiteren Berücksichtigung von Alternativen und dem Verzicht auf frühere Auswahlen zugunsten neuer Möglichkeiten einhergehe. Dagegen sei der Promotion Focus neben einer Verlustfokussierung mit einer eingeengten Berücksichtigung von Alternativen und einer Bindung an vorherige Auswahlen verknüpft (vgl. Liberman, Idson, Camacho \& Higgins, 1999). Molden und Hui (2011) stellten daher die Hypothese auf, dass ein Prevention Focus Personen anfällig gegenüber Eskalationstendenzen bleiben lässt, während ein Promotion Focus diese abschwächen kann. 
Zwei Studien wurden zur Hypothesentestung durchgeführt, wobei nur in der zweiten Untersuchung ein Sunk-Cost-Szenario verwendet wurde. Hierbei handelte es sich um die $\mathrm{SC}^{(+)}$-Version des Airplane-Szenarios von Arkes und Blumer (1985, vgl. Abschnitt 2.1.3.1), in dem die Investitionsentscheidung als abhängige Variable dichotom erhoben wurde (investieren: ja vs. nein). Der regulatorische Fokus wurde in dieser Studie unmittelbar vor der Bearbeitung des Sunk-Cost-Szenarios als dreistufiger Faktor (Promotion vs. Prevention vs. Kontrollgruppe) experimentell induziert. Hierfür absolvierten die Probanden eine fünfminütige Schreibaufgabe, in der sie in der Promotion-Bedingung über ihre „Hoffnungen und Ziele“ und in der Prevention-Bedingung über ihre „Aufgaben und Verpflichtungen“ berichten sollten. Die Probanden der Kontrollgruppe, in der kein regulatorischer Fokus aktiviert wurde, schrieben über ihre „typischen täglichen Aktivitäten“. Bei der eingesetzten Schreibaufgabe handelt es sich um eine Standardmethode zur Manipulation des situativen regulatorischen Fokus, die auch in früheren Studien bereits erfolgreich angewendet wurde (vgl. Freitas \& Higgins, 2002; Freitas, Liberman \& Higgins, 2002; Higgins, Roney, Crowe \& Hymes, 1994; Idson et al., 2004; Liberman et al., 1999; Liberman, Molden, Idson \& Higgins, 2001). Unter Verwendung dieser Methode ließen sich in anderen Forschungsbereichen zudem strukturell ähnliche Effekte für den induzierten regulatorischen Fokus nachweisen, wie für den chronischen regulatorischen Fokus (siehe exemplarisch: Liberman et al., 1999 oder Liberman et al., 2001).

In der Studie von Molden und Hui (2011) investierten die Probanden, bei denen ein Promotion Focus experimentell induziert wurde, signifikant seltener als die Probanden mit einem Promotion Focus oder die Probanden in der Kontrollgruppe. Das Investitionsverhalten unterschied sich zwischen den beiden letztgenannten Faktorstufen nicht voneinander. Außerdem gab es im Gegensatz zu den anderen Versuchsbedingungen nur bei einem Promotion Focus kein signifikantes Überwiegen von Entscheidungen für oder gegen eine 
Investition. Molden und Hui (2011) leiteten hieraus ab, dass ein Promotion Focus hilfreich sei, um versunkene Kosten besser zu ignorieren.

\subsubsection{Rational Thinking Style}

Wong et al. (2008) berichteten eine stärkere Investitionsbereitschaft für Personen mit hohen Ausprägungen auf der Rationalitätsskala aus Pacinis und Epsteins (1999) RationalExperimental Inventory als für Personen mit niedrigen Ausprägungen hierauf. Die Investitionsbereitschaft wurde dabei mithilfe einer modifizierten Version von Arkes und Blumers (1985) Airplane-Szenario erhoben. ${ }^{13}$

Die von den Autoren verwendete Fragebogenskala basiert auf der Cognitive-Experiental Self-Theory von Epstein (1994), die von zwei parallelen, interagierenden Formen der Informationsverarbeitung ausgeht, nämlich einem rationalen und einem erfahrungsbasierten Informationsverarbeitungssystem (vgl. Epstein, 1994; Pacini \& Epstein, 1999; Wong et al., 2008). Von Relevanz für Wong et al. (2008) war allerdings nur der Rational Thinking Style (RTS), also das rationale Informationsverarbeitungssystem. Hierbei handelt es sich um ein inferentielles System, das von dem Verständnis einer Person über die ihr kulturell vermittelten Argumentationsregeln beeinflusst wird und als „,bewusst, relativ langsam, analytisch, primär verbal und relativ affektfrei“ beschrieben wird (Pacini \& Epstein, 1999, S. 972).

Bei der Messung des RTS lässt sich zwischen einer fähigkeitsbasierten und einer motivationalen Komponente unterscheiden: Rational Ability (RA) und Rational Engagement (RE). Personen mit hohen Ausprägungen auf der RA-Skala besitzen im Vergleich zu Personen mit niedrigen Ausprägungen einen stärker analytisch und logisch geprägten Verstand. Sie sind mit höherer Wahrscheinlichkeit in der Lage, Gründe und Erklärungen zu

\footnotetext{
13 Nach Meinung der Autoren hätten sie mit dem Airplane-Szenario Escalation of Commitment (EoC) gemessen und begründen die Verwendung des Szenarios damit, dass dieses bereits in früherer Forschung vielfach zur Untersuchung von EoC eingesetzt worden sei (Wong et al., 2008, S. 253). Diese Einschätzung wird in der vorliegenden Arbeit nicht geteilt, da es sich bei dem Airplane-Szenario wie erläutert um ein klassisches Sunk-Cost-Szenario handelt (vgl. Abschnitt 2.1.2 \& 2.1.3), innerhalb dessen auch bei Wong et al. (2008) die Bereitschaft für die Investition einer festgelegten, nicht aufteilbaren Summe zur Fortsetzung des Projekts gemessen wurde.
} 
verstehen, die ihren Entscheidungen zugrunde liegen. Personen mit hohen Ausprägungen auf der RE-Skala haben im Vergleich zu Personen mit niedrigen Ausprägungen einen stärkeren motivationalen Antrieb analytisch und logisch zu denken (vgl. Wong et al., 2008, S. 250).

Wong et al. (2008) prognostizierten eine höhere Eskalationstendenz für Personen mit hohen Rationalitätsausprägungen im Vergleich zu Personen mit niedrigen Ausprägungen, da erstere die relevanten Informationen tiefgehender verarbeiten würden, auf diese Weise stärkere Überzeugungen für ihre vorherigen Entscheidungen entwickeln und darum eine stärkere kognitive Dissonanz (siehe Festinger, 1957) bei negativem Feedback empfinden als Personen mit niedrigen Rationalitätsausprägungen. Diesen Zusammenhang erwarteten die Autoren sowohl für den RTS, als auch für die beiden Subskalen RA und RE.

Für die vorliegende Arbeit ist im Hinblick auf den Sunk-Cost-Effekt nur die erste Studie von Wong et al. (2008) relevant. Hierin ließen sie ihre Probanden eine modifizierte Version von Arkes und Blumers (1985) Airplane-Szenario bearbeiten (siehe Wong et al., 2008, S. 271). Die Modifikationen beschränkten sich im Wesentlichen darauf, dass in dem Szenario anstelle eines radarunsichtbaren Flugzeugs ein radarunsichtbares Schiff entwickelt wird. Die Höhe der versunkenen Kosten beträgt 5 Millionen Dollar aus einem Budget von 10 Millionen Dollar (anstelle der 9 Millionen Dollar wie bei Arkes \& Blumer, 1985). Über das ursprüngliche Airplane-Szenario hinausgehend, wurde die Information eingefügt, dass mit dem Projekt 600 Millionen Dollar verdient werden können. In diesem Zusammenhang erfolgte eine inter-individuelle Variation positiven vs. negativen Framings, indem entweder die potenziellen Gewinne (positives Framing) oder die potenziellen Verluste (negatives Framing) in den Vordergrund gestellt wurden. Der Faktor Framing sollte eigentlich einer anderen Forschungsfrage dienen und war laut Wong et al. (2008) somit irrelevant für ihre zentrale Untersuchung. Daher wurde das Framing in der Originalstudie als Kontrollvariable behandelt, ebenso wie das Alter, das Geschlecht und das persönliche Verantwortlichkeitsempfinden, welches durch zwei zusätzliche Items erhoben wurde. 
Am Ende des Sunk-Cost-Szenarios sollten die Probanden ihre prozentuale Bereitschaft zur Fortsetzung dieses Projekts (in einer Spanne zwischen 0\% und 100\%) angeben, welche als Kriteriumsvariable diente. Die Messung des RTS und seiner Subskalen RA und RE erfolgte im Anschluss an das Sunk-Cost-Szenario, mithilfe der Rationalitätsskala aus Pacinis und Epsteins (1999) Rational-Experimental Inventory. Darin beziehen sich je zehn Items auf die Subskalen RA und RE (siehe Pacini \& Epstein, 1999, S. 976).

Im Ergebnis zeigten sich signifikante Einflüsse von RTS und RA auf die Investitionsbereitschaft, deren Zusammenhänge jeweils positiv korreliert waren. Ein Zusammenhang zwischen RE und der Investitionsbereitschaft konnte nicht nachgewiesen werden. Die gesteigerte Investitionsbereitschaft durch den Rational Thinking Style war den Autoren zufolge also vor allem auf die Fähigkeitskomponente zum rationalen Denken zurückzuführen (RA), nicht aber auf die motivationale Komponente (RE). 


\subsection{Replizierbarkeit empirischer Befunde}

Die vorliegende Arbeit setzt sich mit grundlegenden Befunden bereits publizierter Studien über die Wirkung von Persönlichkeitsvariablen auf den Sunk-Cost-Effekt auseinander. Das Ziel ist, jene Befunde kritisch zu überprüfen und gegebenenfalls zu erweitern. Die Notwendigkeit einer kritischen Überprüfung ergibt sich insbesondere vor dem Hintergrund der gegenwärtigen Methodendiskussion im Fachbereich der Psychologie, die sich stark mit der Replizierbarkeit empirischer Befunde auseinandersetzt (für einen Überblick siehe Asendorpf et al., 2013; Francis, 2013). In den letzten Jahren stieg nämlich kontinuierlich die Zahl von Nachweisen darüber, dass ein großer Teil der in der Literatur berichteten Befunde gar nicht replizierbar zu sein scheint, was eine regelrechte Replikationskrise in der psychologischen Forschungsgemeinschaft ausgelöst hat. Ein Beispiel ist ein im Jahre 2015 publizierter Bericht der Open Science Collaboration, die Befunde aus 100 psychologischen Studien, veröffentlicht in den besten Fachzeitschriften der Psychologie, auf ihre Replizierbarkeit überprüfte. Von den Studien wiesen 97\% signifikante Resultate mit $p$-Werten $<.05$ aus, aber nur in 36\% aller Fälle ließen sich die Befunde auch replizieren. Bei den restlichen Studien steht hingegen die Möglichkeit falsch positiver Befunde im Raum, also der fälschliche Nachweis von Effekten, die es in Wirklichkeit gar nicht gibt (siehe dazu: Ioannidis, 2005; Simmons, Nelson \& Simonsohn, 2011).

In diesem Unterkapitel werden zunächst verschiedene Ursachen für die Entstehung nicht replizierbarer Befunde vorgestellt, bevor anschließend - im Hinblick auf den empirischen Teil der vorliegenden Arbeit - die Notwendigkeit zur Durchführung von Replikationsstudien erläutert wird.

\subsubsection{Ursachen nicht replizierbarer Befunde}

Der Ausgangspunkt für die gegenwärtige Methodendiskussion waren Aufdeckungen verschiedener Missstände im Jahr 2011 in vorwiegend sozialpsychologischen Studien, 
weswegen Wagenmakers (2012) es plakativ als „Year of Horrors“ für alle Sozialpsychologen bezeichnet. In diesem Jahr erschien unter anderem ein Artikel von Daryl Bem (2011) im Journal of Personality and Social Psychology (JPSP), einer der angesehensten Fachzeitschriften der Sozialpsychologie. Die Aussage des Artikels war, dass Menschen in der Lage seien in die Zukunft zu schauen. Dieser Effekt zeigte sich z.B. in Bems (2011) erstem Experiment am stärksten bei extrovertierten Frauen, die die Position von erotischen Bildern auf der rechten oder linken Seite eines Bildschirms vorhersagen mussten (vgl. Lee \& Wagenmakers, 2014, S. 176). Die Tatsache, dass eine hoch angesehene Fachzeitschrift der Psychologie, mit ansonsten sehr hohen Ablehnungsraten, einen solch fragwürdigen Artikel überhaupt publiziert, war dem Ansehen des gesamten Fachbereichs wenig zuträglich. Ebenso kritisch war die Tatsache, dass das JPSP zunächst alle in der Folge eingereichten Replikationsstudien, die Bems (2011) Originalbefunde nicht replizieren konnten, aufgrund ihrer internen Unternehmenspolitik ablehnte (vgl. Wagenmakers 2012).

Ein nicht replizierbarer Befund kann verschiedene Ursachen haben. Einige von ihnen sollen in den folgenden Abschnitten vorgestellt werden. Im unverfänglichsten Fall liegt ein statistischer Fehler vor. Ungünstiger, da prinzipiell vermeidbar, ist hingegen, wenn der Befund auf unsauberes wissenschaftliches Arbeiten zurückzuführen ist. Im schlimmsten anzunehmenden Fall ist sogar vorsätzlicher Betrug die Ursache.

\subsubsection{Betrugsfälle}

Ebenfalls im Jahr 2011 wurde der bis dato hoch angesehene Wissenschaftler Diederik Stapel überführt, in seinen Studien vorsätzlich Datenbetrug begangen zu haben. Sein Handeln, das er an drei verschiedenen Universitäten praktizierte, blieb über 15 Jahre hinweg unentdeckt (vgl. Schuh, 2014). Drei Untersuchungskommissionen der betroffenen Universitäten aus Amsterdam, Groningen und Tilburg analysierten daraufhin diesen Fall. Dabei konnte in 137 überprüften Publikationen Stapels in 25 Fällen eine Manipulation von Daten nachgewiesen 
werden. In 30 Publikationen waren die Daten sogar frei erfunden! Weiter enthielten auch 10 von 18 seiner betreuten Doktorarbeiten fiktive Daten (vgl. Rauner, 2014). Besonders der Fall von Diederik Stapel hat das Ansehen der Psychologie stark in Mitleidenschaft gezogen, die ansonsten als streng empirisch orientierte Wissenschaft galt. Dass es sich hierbei keineswegs um einen Einzelfall handelt, zeigen weitere nachgewiesene Betrugsfälle, z.B. von den Wissenschaftlern Dirk Smeeters oder Lawrence Sanna.

\subsubsection{Questionable Research Practices und Confirmation Bias}

Datenbetrug ist selbstverständlich die schlimmste anzunehmende Form wissenschaftlichen Fehlverhaltens, da hierbei mutwillig nicht replizierbare Befunde produziert werden. Allerdings müssen diese nicht immer das Produkt vorsätzlichen Fehlverhaltens sein. Auch Fahrlässigkeit, bzw. methodisch unsauberes Arbeiten im Rahmen des Forschungsprozesses, kann nicht replizierbare Befunde generieren. Simmons et al. (2011) beschäftigten sich ausführlich mit dem großen Gestaltungsspielraum, der sich im Rahmen der Datenerhebung, Analyse und Publikation bietet. Vor diesem Hintergrund sprechen die Autoren von „Questionable Research Practices“ (QRPs), von denen einige im Folgenden vorgestellt werden sollen:

Üblicherweise sind den Forschungsprozess begleitend verschiedene Entscheidungen zu treffen, die dem Forscher gewisse Freiheitsgrade bieten. Zum Beispiel könnte im Rahmen einer laufenden Datenerhebung die Entscheidung gefällt werden, mehr Daten zu erheben als geplant, bis ein $p$-Wert $<.05$ erreicht ist, um danach die Erhebung optional zu stoppen. Eine andere denkbare QRP wäre die Erhebung einer Vielzahl abhängiger Variablen, in der Hoffnung, dass sich bei irgendeiner hiervon ein signifikantes Ergebnis findet. Schließlich steigt mit zunehmender Anzahl erhobener Variablen auch die Wahrscheinlichkeit, auf dem in der Forschung allgemein üblichen Signifikanzniveau von $5 \%$ zufällig ein signifikantes Ergebnis zu erzielen (vgl. Simmons et al., 2011). In diesem Fall könnte sich eine 
nachträgliche Hypothesenformulierung als weitere QRP anschließen. Daryl Bem, Autor des bereits angesprochenen fragwürdigen „Wahrsageartikels“, hat an einem im Jahr 1987 erschienenen Leitfaden für junge Sozialforscher mitgewirkt. In dem von ihm verfassten Kapitel gibt er Handlungsempfehlungen für eine Artikelplanung. Gleich zu Beginn des Kapitels empfiehlt er:

„There are two possible articles you can write: (a) the article you planned to write when you designed your study or (b) the article that makes the most sense now that you have seen the results. They are rarely the same, and the correct answer is (b)." (Bem, 1987, S.171-172).

Dass die Anwendung von QRPs, wie eine nachträglichen Hypothesenformulierung, vermeintliche Vorteile mit sich bringe, wurde angehenden Forschern augenscheinlich schon vor 30 Jahren im Studium vermittelt.

Wurde im Verlauf einer Untersuchung wie erwähnt bereits eine Reihe von Variablen erhoben, schließen sich im Rahmen der Datenanalyse vielfältige statistische Möglichkeiten an, den Datensatz auszuwerten. Gelman und Loken (2013) beschreiben dieses als „garden of forking paths“, in dem zumindest einer der vielfältige Auswertungspfade auch zu einem signifikanten Ergebnis führen kann. Zum Beispiel könnte der Erreichung einer Signifikanz durch nachträgliche Kombination oder Transformation der erhobenen Variablen und Bedingungen oder durch Einschaltung beliebiger Kontrollvariablen nachgeholfen werden. Darüber hinaus könnten auch verschiedene Ausschlusskriterien ausprobiert werden, damit einzelne Probanden aus der Analyse herausgenommen werden können, um auf diese Weise signifikante Ergebnisse zu erzielen (vgl. Simmons et al., 2011).

Je mehr Freiheitsgrade es im Forschungsprozess gibt, desto größer ist die Versuchung QRPs anzuwenden. Die Gefahren, die hierdurch entstehen, zeigten Simmons et al. (2011) eindrucksvoll auf. Sie berichteten, dass sich durch die Kombination einiger typischer QRPs mit einer Wahrscheinlichkeit von $61 \%$ ein falsch positiver Befund auf einem Niveau von $p<$ 
.05 erzielen ließe. Jedoch muss hinter dem Ausnutzen von Freiheitsgraden keine böse Absicht stehen; vielmehr liege gemäß der Autoren das Streben nach Hypothesenkonformität und signifikanten Ergebnissen im Naturell eines Forschers. Den Autoren folgend, finden sich in der Literatur diverse Hinweise, dass ein Forscher bei uneindeutigen analytischen Entscheidungen geneigt sein wird, jene Alternative als angemessen zu bewerten, die zu einem signifikanten Ergebnis führt (vgl. z.B. Kunda, 1990).

An dieser Stelle würden die meisten hiermit konfrontierten Wissenschaftler die Anwendung von QRPs vermutlich weit von sich weisen. Dass das Gegenteil in der Forschungspraxis aber in erschreckend hohem Ausmaße der Fall ist, und es sich bei Simmons et al. (2011) Arbeit nicht nur um eine praxisferne Simulation handelt, zeigten John, Loewenstein und Prelec (2012), die die Prävalenz von QRPs gemessen haben. Dazu führten sie eine anonyme Befragung unter 2155 Psychologen durch, in der die persönlichen Erfahrungen mit QRPs erhoben wurden. Abgefragt wurden die Selbsteingeständnisrate der Befragten über die eigene Anwendung von QRPs und die geschätzte Prävalenz für die Anwendung von QRPs im Allgemeinen. In diesem Rahmen zeigte sich, dass QRPs scheinbar durchaus verbreitet sind, mit Selbsteingeständnisraten von teilweise über $50 \%$ für selektives Berichten von abhängigen Variablen oder optionales Stoppen von Datenerhebungen. Deutlich höher lagen die geschätzten Prävalenzraten für diese QRPs mit über 70\%. Selbst das Abrunden von $p$-Werten auf $p=.05$, also ein vorsätzlicher Betrug, wurde noch mit $22 \%$ (Selbsteingeständnisrate) respektive 39\% (Prävalenzrate) bewertet. Eine vollständige Liste der fragwürdigen Forschungspraktiken findet sich bei John et al. (2012), S. 525, Tabelle 1.

\subsubsection{Statistische und menschliche Fehler}

In den letzten beiden Abschnitten wurde die Entstehung nicht replizierbarer Befunde auf vorsätzliches oder fahrlässiges Handeln von Forschern zurückgeführt. Allerdings kann ein solcher Befund auch bei einem korrekten Verhalten im Rahmen der statistischen 
Hypothesenprüfung rein zufällig auftreten. Die Inferenzstatistik unterscheidet dabei zwischen zwei möglichen Fehlerarten: Wird auf dem ausgewählten Signifikanzniveau die Nullhypothese verworfen, obwohl sie in Wirklichkeit gilt und der entsprechende Effekt daher gar nicht existiert, liegt ein „Fehler 1. Art“ (oder Alpha-Fehler) vor, der zu einem falsch positiven Befund führt. Ein „Fehler 2. Art“ (oder Beta-Fehler) liegt hingegen vor, wenn die Nullhypothese beibehalten wird, obwohl sie eigentlich falsch ist, woraus ein falsch negativer Befund resultiert (vgl. Bortz \& Schuster, 2010).

Auch menschliche Fehler im Rahmen der statistischen Hypothesenüberprüfung können eine Quelle für nicht replizierbare Befunde sein. Eines der berühmtesten Beispiele der letzten Jahre hierfür ist der Excel-Gate-Fall der beiden Harvard-Wirtschaftswissenschaftler Reinhart und Rogoff (2010). Diese prüften den Zusammenhang zwischen Wirtschaftswachstum und Staatsverschuldung anhand historischer Daten verschiedener Staaten. Sie kamen zu dem Schluss, dass ab einem Schuldenstand oberhalb von 90\% das Wirtschaftswachstum eines Staates drastisch reduziert werde. Ihr Artikel erschien kurz nach Beginn der Griechenlandkrise 2010 und fand weltweite Beachtung, vor allem als Rechtfertigungsgrund von Regierungen für die Durchsetzung von Sparpolitiken. In der darauf folgenden Zeit entstand jedoch das Problem, dass Reinharts und Rogoffs (2010) zentraler Befund anhand vergleichbarer Daten nicht repliziert werden konnte, weswegen sie Mitarbeitern der Universität aus Massachusetts einen Blick in ihre Originaldaten gestatteten. In diesem Zuge wurde aufgedeckt, dass Reinhart und Rogoff teilweise Daten übersehen hatten, die somit nicht in ihre Berechnungen eingeflossen sind. Außerdem enthielten die Berechnungen selbst fragwürdige statistische Verfahren. Darüber hinaus waren ihnen auch noch Codierungsfehler in Excel unterlaufen (vgl. Krugman, 2013). In diesem Fall lagen also schlichte menschliche Fehler vor, deren Folgen eine enorme Tragweite für Staaten in aller Welt hatten. 


\subsubsection{Publication Bias}

Wagenmakers (2012) berichtete wie bereits erwähnt, dass vom JPSP alle Manuskripte anderer Forscher abgelehnt wurden, die über gescheiterte Replikationsversuche von Bems (2011) „Wahrsageartikel“ berichten wollten. Der Grund hierfür war, dass das JPSP den Grundsatz verfolgte, keine bloßen Replikationsstudien zu publizieren. Tatsächlich ist diese Unternehmenspolitik exemplarisch für die meisten Fachzeitschriften (vgl. Nosek, Spies \& Motyl, 2012). Makel, Plucker und Hegarty (2012) kamen zu dem Ergebnis, dass nur 1,07\% aller Artikel, die in den 100 wichtigsten psychologischen Fachzeitschriften (bemessen nach ihrem 5-Jahres Impact-Faktor) zwischen 1900 und 2010 veröffentlicht wurden, überhaupt Replikationsstudien waren. Diese Selektion setzt jedoch falsche Anreize für Forscher, für deren Karriereverlauf erfolgreiche Publikationen essenziell sind. Wegen der schlechten Publizierbarkeit werden die meisten Forscher ohnehin geneigt sein, von der Durchführung einer Replikationsstudie abzusehen. Sofern sie diese doch durchführen, aber negative Befunde erzielen, wird oftmals gar nicht erst der Versuch unternommen, die Studie zu publizieren. In Anlehnung an den „File-Drawer Effekt“ von Rosenthal (1979), lassen sie die Studien metaphorisch lieber in ihren Schubladen verschwinden, vor allem, wenn es sich um gescheiterte Replikationsstudien eigener Befunde handelt (vgl. Makel et al., 2012).

Die Problematik ist bekannt unter dem Namen „Publication Bias“ (z.B. Francis, 2012), der allerdings nicht alleine auf Replikationsstudien bezogen ist. Im vorangegangenen Abschnitt wurde bereits die allgemein akzeptierte statistische Irrtumswahrscheinlichkeit von 5\% erwähnt. Im Zusammenhang mit dem Publication Bias sei nun ergänzt, dass mit hinreichend hoher Anzahl ähnlich durchgeführter Studien auch die Wahrscheinlichkeit steigt, in irgendeiner davon zufällig einen Alpha-Fehler zu generieren. Wird in der Folge genau diese eine Studie mit dem falsch positiven Befund publiziert, während die konträren Befunde aller übrigen Studien im Verborgenen bleiben, sei es durch das Verschwinden der Studienergebnisse in Schubladen oder durch die Ablehnung durch Fachzeitschriften, verzerrt 
dieses den Gesamteindruck von der Realität (vgl. Bakker, von Dijk \& Wicherts, 2012; Francis, 2012, 2013; Ioannidis \& Trikalinos, 2007).

Dass in Fachzeitschriften tatsächlich fast ausschließlich positive Befunde berichtet werden, zeigt eine Studie von Fanelli (2010), in der 2434 Publikationen aus 20 empirischen Forschungsfeldern miteinander verglichen wurden. Der Prozentsatz für bestätigte Hypothesen im Bereich Psychologie / Psychiatrie lag darin bei 92\%. Dieses war der höchste Wert unter allen Forschungsfeldern $(M=84 \%)$. Zudem sei die Bestätigungsrate zwischen den Jahren 1990 und 2007 stark angestiegen berichtet Fanelli (2012). Eine derartig hohe Bestätigungsrate liege laut Asendorpf et al. (2013) allerdings weit über jenem Wert, den man in Anbetracht der typischen Effektgrößen und Teststärken von psychologischen Studien erwarten würde.

\subsubsection{Notwendigkeit zur Durchführung von Replikationsstudien}

In der Wissenschaft herrscht die Auffassung vor, dass Publikationszahlen und Journal Prestige der entscheidende Schlüssel zum Erfolg eines Forschers sind, z.B. bei der Besetzung von Universitätsprofessuren. Außerdem sieht die Unternehmenspolitik vieler hochrangiger Fachzeitschriften vor, nur neuartige und positive Befunde $\mathrm{zu}$ publizieren (vgl. Hicks, Wouters, Waltman, de Rijcke, \& Rafols, 2015; Nosek et al., 2012). Folglich vergleichen Bakker et al. (2012) Wissenschaft sogar mit einem Spiel, dessen dominante Regel es ist, so viele signifikante Befunde wie möglich zu sammeln. In diesem Spiel müssen die Akteure allerdings befürchten, Wettbewerbsnachteile zu erlangen, sofern sie fair agieren. Die genannten Rahmenbedingungen hinsichtlich der Reputation bilden somit einen Nährboden für die Produktion von nicht replizierbaren Befunden durch Ursachen, wie in den vorherigen Abschnitten erläutert. Im unverfänglichsten Fall ist die Entstehung nicht replizierbarer Befunde rein statistisch begründet; viel entscheidender ist jedoch, dass ihre Quelle auch der Forscher selbst sein kann. Menschliche Fehler, unsauberes Arbeiten im Forschungsprozess oder sogar vorsätzlicher Datenbetrug wurden hier als mögliche Ursachen genannt, flankiert 
durch den Publication Bias. Die Gefahr ist groß, dass alles zusammen zu einer Abnahme des Wahrheitsgehalts unseres geteilten Wissens führt (vgl. Nelson, Simmons \& Simonsohn, 2012).

Unwahres geteiltes Wissen, das auf nicht replizierbaren Befunden beruht, kann weitreichende Konsequenzen für Wissenschaft und Praxis mit sich bringen. In der Wissenschaft werden Forscher möglicherweise über Jahre hinweg damit beschäftigt sein, Folgestudien für bereits publizierte Befunde zu konzipieren und bei ihren eigenen Erhebungen schon an der Replikation der Grundbefunde scheitern - in Unkenntnis darüber, dass es sich bei den Ergebnissen der Originalstudien in Wirklichkeit um falsch positive Befunde handelt. Werden diese gescheiterten Replikationsberichte daraufhin nicht publiziert, z.B. aufgrund der Tatsache, dass nicht geglückte Replikationsversuche schwer in angesehenen Fachzeitschriften $\mathrm{zu}$ veröffentlichen sind, wird es in der Folge immer weitere Forscher geben, die in Unwissenheit über die tatsächliche Anzahl bereits durchgeführter, erfolgloser Replikationsstudien eigene Replikationsversuche starten. Daraus resultieren auf Dauer eine Verschwendung finanzieller und zeitlicher Ressourcen sowie eine Benachteiligung individueller Karriereverläufe. Aus der Metaperspektive wird klar, dass sich falsch positive Befunde, sofern einmal publiziert, über viele Jahre hinweg hartnäckig in der Fachliteratur halten können (vgl. Asendorpf et al., 2013; Greitemeyer, 2014; Simmons et al., 2011).

In der Praxis kann sogar Gefahr für Leib und Leben entstehen, wenn z.B. die Wirksamkeit medizinischer Präparate vermeintlich durch Studien belegt wurde, diese Präparate tatsächlich aber unwirksam oder sogar schädlich sind (vgl. Prinz, Schlange \& Asadullah, 2011). Selbst dann, wenn alle wissenschaftlichen Untersuchungen eines Effekts bereits eingestellt wurden, können falsch positive Befunde in der Praxis weiter Einfluss nehmen. Als Beispiel nennen Bakker et al. (2013) den Mozart-Effekt, wonach das Hören klassischer Musik zu einer Steigerung des IQs führen soll. Obwohl der Effekt längst widerlegt wurde, habe er weiterhin 
Bestand im kollektiven Gedächtnis und sorge nach wie vor für ein reichhaltiges Angebot entsprechender Musik-CDs bei Händlern.

Zum aktuellen Zeitpunkt ist unklar, wie viele bekannte Effekte aus der Literatur derzeit auf nicht replizierbaren Befunden beruhen. Aufgrund ihrer verhängnisvollen Konsequenzen sollte der Fokus der psychologischen Forschung nun vor allem auf der Aufdeckung dieser Befunde liegen. Dies gelingt nur durch gewissenhaft durchgeführte Replikationsstudien. Nach Meinung von Asendorpf et al. (2013) bilden Replikationsstudien sogar das „Herzstück“ jeder empirischen Wissenschaft. Den Autoren folgend, sei es eine wichtige Voraussetzung für die Generalisierbarkeit eines Effekts, dass sein Auftreten auch in anderen zufälligen Samples nachgewiesen werden kann, unter Einschluss anderer Versuchspersonen, Situationen, Operationalisierungen oder Zeitpunkte. Sofern die Replikationsstudien ansonsten in bestmöglicher Übereinstimmung mit der Originalstudie durchgeführt werden, und daraufhin keine bedeutenden Unterschiede zwischen den Befunden aus der Original- und Replikationsstudie nachweisbar sind, die höchstens auf unsystematischen Fehlern, Stichprobenausreißern oder einzelnen Werteunterschieden im Design beruhen, könne man eine Replikation als geglückt betrachten (vgl. Asendorpf et al., 2013). Replikationsstudien liefern also wichtige Indizien hinsichtlich der Generalisierbarkeit bzw. über die tatsächliche Existenz oder Nichtexistenz eines Effekts und dürfen nicht in Schubladen verschwinden, sofern sie den Originalbefund bei gewissenhafter Versuchsdurchführung nicht replizieren können. Daher kommt der kritischen Überprüfung der Persönlichkeitsvariablen im Rahmen der vorliegenden Arbeit, deren Einflüsse auf den Sunk-Cost-Effekt bisher einzig durch ihre jeweiligen Originalstudien belegt sind, eine große Bedeutung zu. 


\section{Zielsetzungen der Arbeit}

Der Sunk-Cost-Effekt besitzt eine hohe Relevanz im täglichen Leben und kann zum Teil weitreichende Konsequenzen nach sich ziehen. In Abschnitt 2.2.2 wurden daher empirische Arbeiten vorgestellt, die nahelegen, dass Handlungs- und Lageorientierung (van Putten et al., 2010), Optimismus und Pessimismus (Juliusson, 2006), der regulative Fokus: Promotion und Prevention (Molden \& Hui, 2011) sowie der Rational Thinking Style (Wong et al., 2008) darüber mitbestimmen, ob und wie anfällig Entscheidungsträger für den Sunk-Cost-Effekt sind. Hierdurch könnten sich in der Praxis Möglichkeiten ergeben, die Wahrscheinlichkeit für Sunk-Cost-Effekte zu reduzieren, z.B. durch eine entsprechende Personalauswahl.

Die Voraussetzung für eine solche Generalisierbarkeit ist allerdings, dass die Originalbefunde auch replizierbar sind. Im Fall der genannten Persönlichkeitsvariablen ist die Replizierbarkeit aber noch nicht geklärt, da die Befunde bisher einzig durch ihre jeweiligen Originalstudien belegt wurden. Aus diesem Grund besteht, insbesondere vor dem Hintergrund der bestehenden Replikationskrise, die Notwendigkeit zur Durchführung von Replikationsstudien, um die Belastbarkeit der publizierten Effekte sicherzustellen (vgl. Abschnitt 2.3). Eine zentrale Zielsetzung der vorliegenden Arbeit ist es daher, die Originalbefunde der vorgestellten Persönlichkeitseffekte durch direkte Replikationsstudien kritisch zu überprüfen. Im Sinne einer exakten Replikation werden diese Untersuchungen so nahe wie möglich an den jeweiligen Originalstudien ausgerichtet.

Im Fall einer erfolgreichen Sicherstellung der Replizierbarkeit der Originalbefunde, sollen diese erweitert werden. Da in den Originalstudien nicht zwischen dem Sunk-Cost-Effekt infolge einer irrationalen Reinvestition und infolge eines irrationalen Abbruchs differenziert wurde, ist derzeit noch unklar, ob die Persönlichkeitsvariablen je nach Situation spezifisch die Neigung zum Sunk-Cost-Effekt reduzieren können oder in den Studien stattdessen pauschal die Investitionsneigung veränderten. In letzterem Fall bestünde in der Praxis die Gefahr, dass 
profitable Projekte frühzeitig abgebrochen werden, obwohl eine Fortführung eigentlich empfehlenswert wäre. Details zur Umsetzung der entsprechenden Untersuchungen werden allerdings erst dargestellt, wenn die Replizierbarkeit der jeweiligen Originalbefunde sichergestellt werden konnte.

Eine weitere Zielsetzung ist, in Form einer integrativen Replikationsstudie zu überprüfen, ob die berichteten Effekte der Variablen auch dann noch feststellbar sind, wenn die jeweils anderen Persönlichkeitsvariablen simultan mit erfasst werden. Dieses trägt der Tatsache Rechnung, dass das Entscheidungsverhalten von mehreren Persönlichkeitsvariablen gleichzeitig beeinflusst werden kann. Es wäre nämlich denkbar, dass aufgrund von Interkorrelationen bestimmte Persönlichkeitsvariablen ihren prädiktiven Wert einbüßen, sobald andere Variablen ihrerseits einen Einfluss auf den Sunk-Cost-Effekt ausüben. Im Gegensatz zur vorgenannten Erweiterung der Originalbefunde, hinsichtlich eines differenziellen Entscheidungsverhaltens, ist diese Untersuchung auch dann noch relevant, wenn die direkten Replikationsversuche scheitern sollten. In dem Fall stellt die integrative Replikationsstudie nämlich vordergründig einen weiteren, methodisch veränderten Replikationsversuch der Originalbefunde im Rahmen der vorliegenden Arbeit dar. Um die simultane Erhebung aller Persönlichkeitsvariablen adäquat umsetzen zu können, werden diese gemeinsam auf Trait-Ebene gemessen.

Am Ende dieser Arbeit sollen also mindestens zwei Replikationsversuche zu jeder Persönlichkeitsvariable auf Trait- bzw. State-Ebene vorliegen, um möglichst genaue Aussagen über die Belastbarkeit der Originalbefunde treffen zu können. Für die Variablen Handlungs- und Lageorientierung sowie den Rational Thinking Style wurde daher jeweils nur eine direkte Replikationsstudie durchgeführt, da die integrative Replikationsstudie den zweiten Replikationsversuch auf Trait-Ebene darstellt. Für die Variablen Optimismus vs. Pessimismus sowie den regulatorischen Fokus ist letzteres nicht der Fall, sodass hierfür jeweils zwei direkte Replikationsstudien durchgeführt wurden. 


\section{Direkte Replikationsstudien}

In diesem Kapitel werden die direkten Replikationsstudien berichtet, mit denen die Persönlichkeitseffekte der in Abschnitt 2.2.2 vorgestellten Originalarbeiten auf ihre Reliabilität überprüft werden sollten. Diese Überprüfung war wie erwähnt insbesondere vor dem Hintergrund der gegenwärtigen Replikationskrise notwendig, da die publizierten Effekte bisher einzig durch ihre jeweiligen Originalstudien belegt sind.

Um exakte Replikationen $\mathrm{zu}$ ermöglichen wurde versucht, alle Aspekte der Originalstudien so genau wie möglich nachzustellen. Das heißt, es wurden die gleichen Materialien, Operationalisierungen und vergleichbare Stichprobenzusammensetzungen verwendet. Außerdem wurde durchweg ohne Täuschung der Probanden und ohne erfolgsabhängige Versuchspersonenvergütung gearbeitet, da ein solches Vorgehen auch in den jeweiligen Originalarbeiten nicht berichtet wurde. Für die Replikationsstudien wurden mindestens gleich große Stichprobenumfänge wie in den Originalstudien gewählt, in den meisten Fällen sogar deutlich größere. Da die Untersuchungsbedingungen ansonsten unverändert blieben, kann so näherungsweise von einer höheren Teststärke der Replikationsstudien gegenüber den Originalstudien ausgegangen werden. Hierdurch erhöht sich die Wahrscheinlichkeit, tatsächlich existierende Effekte statistisch nachweisen zu können. Gleichzeitig vermindert sich die Wahrscheinlichkeit, potenzielle Nullbefunde auf mangelnde Teststärke zurückführen zu müssen (vgl. Asendorpf et al., 2013; Bortz \& Schuster, 2010; Cohen, 1962). Ein nomineller Vergleich der exakten Teststärken wurde in den folgenden Abschnitten nicht vorgenommen, da die hierfür notwendigen Effektgrößen in den Originalarbeiten nicht berichtet wurden.

Die direkten Replikationsstudien werden in den folgenden Unterkapiteln konstruktweise vorgestellt, bevor im Anschluss ein gemeinsames Zwischenfazit gezogen wird. 


\subsection{Handlungs- und Lageorientierung}

Wie in Abschnitt 2.2.2.1 dargestellt, berichteten van Putten et al. (2010) einen moderierenden Einfluss von Trait-Handlungs- und Lageorientierung (Kuhl \& Beckmann, 1994a) auf den Sunk-Cost-Effekt. In ihrer Studie zeigten eher lageorientierte Probanden eine stärkere Sunk-Cost-Neigung im Airplane-Szenario von Arkes und Blumer (1985) als eher handlungsorientierte Probanden. Handlungsorientierung eliminierte zwar nicht in allen Fällen den Sunk-Cost-Effekt, reduziere laut der Autoren jedoch generell die Wahrscheinlichkeit in fehlgehende Projekte zu investieren.

\subsubsection{Methode}

\subsubsection{Stichprobe und Design}

An der vorliegenden Replikationsstudie nahmen 75 Studierende der Georg-AugustUniversität Göttingen teil. Das entspricht exakt der Stichprobengröße von van Putten et al. (2010). Hiervon waren 54 Probanden weiblich (72\%) und 21 männlich (28\%). Das Durchschnittsalter betrug 22,16 Jahre $(S D=3,09)$, in einer Spanne zwischen 18 und 30 Jahren.

Das Versuchsdesign entsprach ebenfalls genau dem der Originalstudie. Die versunkenen Kosten wurden als zweistufiger Zwischensubjektfaktor variiert (vorhanden vs. nicht vorhanden) und die chronischen Ausprägungen von Handlungs- und Lageorientierung auf Trait-Ebene erfasst. Als Kriteriumsvariable diente die dichotome Investitionsentscheidung (ja vs. nein) innerhalb des Airplane-Szenarios (Arkes \& Blumer, 1985).

\subsubsection{Durchführung und Material}

Wie bei van Putten et al. (2010) wurden die Erhebungen der vorliegenden Replikationsstudie im Abstand von einer Woche und im Rahmen einer größeren Untersuchung durchgeführt. Die Probanden wurden jeweils direkt im Anschluss an die 
größere Untersuchung gefragt, ob sie noch an einer weiteren Studie zum Thema „Persönlichkeitsmessung und Entscheidungsverhalten“ teilnehmen möchten. Dabei informierte die Versuchsleitung auch über die zwei Messzeitpunkte im Abstand von einer Woche und die festgelegte Versuchspersonenvergütung in Höhe von fünf Euro. In Abhängigkeit der Teilnehmerzahl der vorangegangenen Untersuchung, fanden sich pro Session bis zu drei Freiwillige für die vorliegende Sunk-Cost-Studie, die ihre Fragebögen anschließend in einem gemeinsamen Laborraum bearbeiteten.

Im ersten Schritt wurde den Probanden von der Versuchsleitung ein individueller Versuchspersonencode zugewiesen und auf einem separaten Zettel ausgehändigt. Der Code diente der Identifikation der Person bei der zweiten Messung in der Folgewoche sowie der korrekten Zuordnung aller Versuchsmaterialien im Rahmen der Auswertung. Daher wurde auch ein entsprechendes Feld auf den Fragebögen von der Versuchsleitung mit dem jeweiligen Code versehen.

Im zweiten Schritt wurde das Versuchsmaterial ausgehändigt. Dieses bestand aus einem dreiseitigen Fragebogen mit allgemeinen Instruktionen auf der ersten Seite, die noch einmal über Ablauf, Bezahlung und Anonymität informierten. Die gleiche Seite enthielt auch demografische Abfragen über das Alter, das Geschlecht, das Studienfach und die Semesteranzahl. Im direkten Anschluss folgten die Fragen zur Erfassung der Handlungs- und Lageorientierung auf Trait-Ebene. Hierbei kamen die gleichen 24 Items des ACS-90 Fragebogens zum Einsatz wie bei van Putten et al. (2010), jedoch in deutscher Originalfassung (HAKEMP 90, vgl. Kuhl \& Beckmann, 1994a; siehe Anhang A). Pro Item mussten sich die Probanden für eine von zwei Antwortmöglichkeiten entscheiden, von denen sich jeweils eine auf Handlungsorientierung und die andere auf Lageorientierung bezog (z.B. „Wenn meine Arbeit als völlig unzureichend bezeichnet wird, dann: a) lasse ich mich davon nicht lange beirren; b) bin ich zuerst wie gelähmt"“). Nach der Bearbeitung des Fragebogens 
dankte die Versuchsleitung den Probanden und vereinbarte mit ihnen einen Termin für die zweite Erhebung, eine Woche später.

Dieser zweite Termin diente der Messung der Sunk-Cost-Neigung. Als die Probanden hierzu in das Labor zurückkehrten, mussten sie sich zunächst mit ihrem Versuchspersonencode ausweisen. Die daraufhin ausgehändigten Fragebögen enthielten eine deutsche Übersetzung des Airplane-Szenarios von Arkes und Blumer (1985, Experiment 3; siehe Anhang A), entweder in der Version mit oder ohne versunkene Kosten. Die Reihenfolge der Fragebögen wurde zuvor zufällig gemischt, sodass die Aufteilung der Probanden auf beide Faktorstufen im Rahmen der Fragebogenverteilung randomisiert erfolgte.

Jeder Fragebogen umfasste jeweils zwei Seiten. Die erste Seite enthielt wieder schriftliche Instruktionen mit Informationen über den Ablauf, die Bezahlung und die Anonymität. Auf der gleichen Seite bearbeiteten die Probanden auch das Airplane-Szenario. Entsprechend der Versuchsdarstellung des Originalartikels, waren hiermit alle Daten wie in der Originalstudie erhoben. Ergänzend folgte auf der zweiten Seite noch ein Abschlussfragebogen. Dieser enthielt, um sicher zu gehen, dass die Teilnehmer über keinerlei Hintergrundwissen bzgl. des Sunk-Cost-Effekts verfügten, drei Abfragen: Erstens, ob in der Vergangenheit schon einmal an einer ähnlichen Studie teilgenommen wurde und zweitens, sofern zutreffend, worum es darin inhaltlich ging und wie diese ablief. Bei der dritten Frage sollten die Teilnehmer im Sinne einer Verdachtskontrolle niederschreiben, worum es ihrer Meinung nach in der vorliegenden Untersuchung ging. Als letztes wurden die Probanden gebeten ihre E-MailAdresse anzugeben, um später nähere Informationen über die Hintergründe der Untersuchung zu erhalten.

Nach Abgabe der Fragebögen erhielten die Probanden die festgelegte Vergütung in Höhe von fünf Euro und wurden von der Versuchsleitung mit Dank verabschiedet. Die Informationen über die Hintergründe der Untersuchung wurden nach Abschluss aller Erhebungen schriftlich an die angegebenen E-Mail-Adressen in Blindkopie versendet. 


\subsubsection{Ergebnisse}

Die Auswertung wurde genauso durchgeführt, wie bei van Putten et al. (2010) berichtet. Die abhängige Variable „Investitionsentscheidung“ wurde mit „0“ (nicht investieren) und „1“ (investieren) codiert, die unabhängige Variable „,versunkene Kosten“ ebenfalls mit „,“ $\left(\mathrm{SC}^{(-)}\right)$ und „1“ $\left(\mathrm{SC}^{(+)}\right)$. Bei der Auswertung des HAKEMP-90 wurden lageorientierte Antworten mit „0“ und handlungsorientierte Antworten mit „,“ codiert und anschließend aufsummiert. Höhere Ausprägungen dieser Summenwerte spiegeln daher einen höheren Grad der Handlungsorientierung wider. Die Summenwerte, mit möglichen Ausprägungen zwischen 0 und 24, wurden wie in der Originalstudie anschließend auf ihren Mittelwert $(M=9.64, S D=$ 3.96) zentriert. Daraus ergab sich das Ausmaß der Handlungs- und Lageorientierung in Form eines Kontinuums. Probanden mit negativen Werten repräsentieren dabei eine stärkere Lageorientierung und Probanden mit positiven Werten eine stärkere Handlungsorientierung. Der Wert 0 entspricht einem mittleren Grad der Handlungs- und Lageorientierung.

Analog zur Originalstudie wurde eine binär-logistische Regressionsanalyse durchgeführt. Um einen direkten Vergleich zu den Ergebnissen der Originalstudie zu ermöglichen, werden die berichteten Effekte von van Putten et al. (2010) in Tabelle 1 zusammengefasst.

Results of a logistic regression analysis

\begin{tabular}{l|l}
$\begin{array}{l}\text { Sunk Cost } \\
\text { Sunk Cost } * \text { Action orientation }\end{array}$ & $B=-1.90, S E=.54 ; \chi^{2}(1, N=75)=12.34, p<.001$ \\
\hline & $B=1.16, S E=.57 ; \chi^{2}(1, N=75)=4.18, p=.04$ \\
\hline $\begin{array}{l}\text { Results of simple slope analyses } \\
\text { State (1 SD below the mean) }\end{array}$ & $B=-3.06, S E=.87 ; \chi^{2}(1, N=75)=12.40, p<.001$ \\
\hline Action (1 SD above the mean) & $B=-.746, S E=.67 ; \chi^{2}(1, N=75)=1.14, p<.29$ \\
\hline
\end{tabular}

Tabelle 1: Befunde der Originalstudie von van Putten et al. (2010). Die dichotome Investitionsentscheidung stellte hier die Kriteriumsvariable dar.

Wie in der Originalstudie konnte auch in der Replikationsstudie ein signifikanter Haupteffekt für die versunkenen Kosten nachgewiesen werden $(B=3.62, S E=.71$; $\left.\chi^{2}[1, N=75]=26.24, p<.001, \mathrm{OR}=37.31\right)$. Demzufolge investierten die Probanden auf der Stufe vorhandener versunkener Kosten häufiger als auf der Stufe ohne versunkene Kosten 
$\left(M_{(S C+)}=83,8 \%\right.$ vs. $\left.M_{(S C-)}=13,2 \%\right)$. In Abschnitt 2.2.2.1 wurde bereits dargelegt, dass von van Putten et al. (2010) ein negativer Regressionskoeffizient $B$ berichtet wurde, was inhaltlich genau das Gegenteil bedeuten würde, nämlich dass ihre Probanden bei vorhandenen versunkenen Kosten weniger investierten als ohne versunkene Kosten. Dieses Verhalten widerspräche sowohl den übrigen Ausführungen der Autoren als auch dem grundlegenden Befund von Arkes und Blumer (1985). Darum ist an dieser Stelle festzuhalten, dass der Effekt der vorliegenden Replikationsstudie in die richtige Richtung wies.

Die zentrale Interaktion zwischen den versunkenen Kosten und Handlungs- und Lageorientierung, wie sie in der Originalstudie auftrat, zeigte sich in der Replikationsstudie allerdings nicht $\left(B=.23, S E=.18 ; \chi^{2}[1, N=75]=1.60, p=.206, \quad \mathrm{OR}=1.26\right)$. Um die Behauptung zu untermauern, dass es sich bei dem Persönlichkeitskonstrukt tatsächlich um eine Moderatorvariable für den Sunk-Cost-Effekt handelt, wäre der Nachweis eines solchen Interaktionseffekts jedoch essentiell gewesen. Wie in der Originalstudie sollten separate Simple-Slope-Analysen auch in der vorliegenden Replikationsstudie näheren Aufschluss über die jeweilige Sunk-Cost-Neigung für beide Persönlichkeitsausprägungen liefern. Die Resultate der Analysen aus der Originalstudie und der Replikationsstudie werden in Abbildung 1 und 2 grafisch gegenübergestellt.
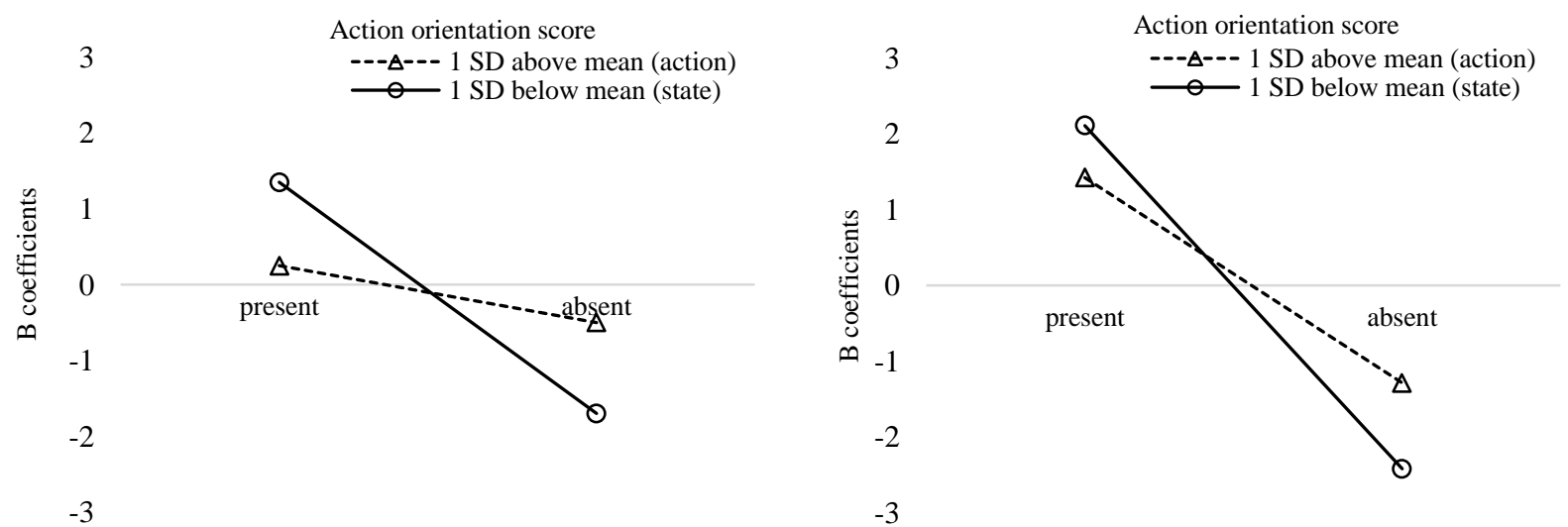

Abbildung 1: Simple-Slope-Analysen der Originalstudie: Vergleich der B-Koeffizienten (logarithmierte Odds Ratios) der versunkenen Kosten für Personen mit hohen und niedrigen Ausprägungen der Handlungs- und Lageorientierung.

Abbildung 2: Simple-Slope-Analysen der Replikationsstudie: Vergleich der B-Koeffizienten (logarithmierte Odds Ratios) der versunkenen Kosten für Personen mit hohen und niedrigen Ausprägungen der Handlungs- und Lageorientierung. 
Das grafische Muster der Simple-Slope-Analysen aus der Originalstudie konnte in der vorliegenden Replikationsstudie näherungsweise reproduziert werden. ${ }^{14}$ In der Tendenz waren auch hier lageorientierte Probanden etwas anfälliger für den Sunk-Cost-Effekt als die Handlungsorientierten. Im Gegensatz zur Originalstudie zeigten sich aber beide Sunk-CostEffekte signifikant, also sowohl für Lageorientierung (eine SD unter dem Mittelwert; $B=4.53, S E=1.15 ; \chi^{2}[1, N=75]=15.57, \quad p<.001, \quad$ OR $\left.=92.55\right)$ als auch für Handlungsorientierung (eine SD über dem Mittelwert; $B=2.71, S E=.84 ; \chi^{2}[1, N=75]=$ 10.31, $p=.001, \mathrm{OR}=15.04)$. Auf Basis der vorliegenden Ergebnisse kann daher nicht festgestellt werden, dass Handlungsorientierung wie in der Originalstudie mit einer gewissen Robustheit gegenüber dem Sunk-Cost-Effekt einherginge. An dieser Stelle irritieren wiederum die negativen Regressionskoeffizienten $B$ der Simple Slopes aus der Originalstudie, die in der vorliegenden Replikationsstudie hingegen positiv ausfielen und somit im Einklang mit Arkes und Blumer (1985) stehen.

Zur weiteren Veranschaulichung ergänzten van Putten et al. (2010) die folgende Tabelle 2. Darin ist, nach Durchführung eines Mediansplits, der Anteil jener Probanden aufgeführt, die sich in den jeweiligen Bedingungen für eine Folgeinvestition entschieden haben. Für einen direkten Vergleich zeigt Tabelle 3 die entsprechenden Resultate der Replikationsstudie.

\footnotetext{
${ }^{14}$ Für einen besseren Vergleich der Grafiken wurde die Skalierung in Abbildung 1, die im Originalartikel von 2 bis 2 reichte, auf -3 bis 3 erweitert. Da bei van Putten et al. (2010) leider keine exakten numerischen Werte angegeben waren, wurden die Datenpunkte aus der Originalgrafik bestmöglich übernommen.
} 
Tabelle 2: Anzahl der Probanden in der Originalstudie von van Putten et al. (2010), die sich für eine Folgeinvestition entschieden.

\begin{tabular}{lrc}
\hline & \multicolumn{2}{c}{ Sunk Cost } \\
\cline { 2 - 3 } & Present & Absent \\
\hline State (Below median) & $16(80 \%)$ & $4(20 \%)$ \\
Action (Above median) & $9(53 \%)$ & $6(33 \%)$ \\
\hline
\end{tabular}

Originalstudie $($ Median = 11); Der Einfluss der versunkenen Kosten auf die Investitionsneigung war bei Lageorientierung $\left(\chi^{2}[1, N=40]=14.40\right.$, $p<.001)$, aber nicht bei Handlungsorientierung $\left(\chi^{2}[1, N=35]=1.73, p=.24\right)$ signifikant.
Tabelle 3: Anzahl der Probanden in der vorliegenden Replikationsstudie, die sich für eine Folgeinvestition entschieden.

\begin{tabular}{lcc}
\hline & \multicolumn{2}{c}{ Sunk Cost } \\
\cline { 2 - 3 } & Present & Absent \\
\hline State (Below median) & $22(85 \%)$ & $3(21 \%)$ \\
Action (Above median) & $9(82 \%)$ & $2(8 \%)$ \\
\hline
\end{tabular}

Replikationsstudie (Median = 9); Der Einfluss der versunkenen Kosten auf die Investitionsneigung war bei Lageorientierung $\left(\chi^{2}[1, N=40)=15.50\right.$, $p<.001, \phi=.62]$ und Handlungsorientierung $\left(\chi^{2}[1\right.$, $N=35]=18.90, p<.001, \phi=.74)$ signifikant

Die Tabellen verdeutlichen noch einmal, warum der entscheidende Interaktionseffekt in der vorliegenden Replikationsstudie ausgeblieben ist. Rein deskriptiv ist in beiden Studien jeweils eine stärkere Investitionsneigung in den $\mathrm{SC}^{(+)}$-Bedingungen ablesbar als in den $\mathrm{SC}^{(-)}$Bedingungen. Jedoch wurde dieser Unterschied für Handlungsorientierung in der Originalstudie nicht signifikant. In der Replikationsstudie zeigte sich hingegen sowohl für Handlungs- als auch für Lageorientierung ein signifikanter Sunk-Cost-Effekt in dieser ergänzenden Analyse. ${ }^{15}$

Van Putten et al. (2010) stellten in Bezug auf Tabelle 2 ebenfalls heraus, dass auf Stufe hoher versunkener Kosten die handlungsorientierten Probanden weniger investitionsfreudig waren als die Lageorientierten (53\% vs. 80\%). Für diesen Unterschied berichteten die Autoren einen marginalen Effekt $\left(\chi^{2}[1, N=37]=3.07, p=.08\right)$. In der Replikationsstudie war der Unterschied mit $82 \%$ gegenüber $85 \%$ nicht signifikant $\left(\chi^{2}[1, N=37]=.05, p=.833, \phi=-\right.$ .04). Auf der Stufe ohne versunkene Kosten war weder in der Originalstudie $\left(\chi^{2}[1, N=38]=\right.$

15 Nach dem Mediansplit ergab sich eine Aufteilung von 35 handlungsorientierten und 40 lageorientierten Probanden, genau wie in der Originalstudie. Allerdings führte der Mediansplit zu einem größeren Ungleichgewicht zwischen den Zellen hinsichtlich der versunkenen Kosten als in der Originalstudie, mit 26 lageorientierten und nur 11 handlungsorientierten Probanden in den entsprechenden $\mathrm{SC}^{(+)}$-Bedingungen. Da der Median (=9) in der Replikationsstudie etwas niedriger ausfiel als in der Originalstudie, wurde zum Vergleich auch eine Berechnung mit dem Originalmedian $(=11)$ durchgeführt. Auch hierbei zeigten sich zwei signifikante Sunk-Cost-Effekte für beide Persönlichkeitsausprägungen (Lageorientierung: $\mathrm{SC}^{(+)}=23$ [82\%] vs. $\mathrm{SC}^{(-)}=3$ $[13 \%] ; \chi^{2}[1, N=51]=24.13, p<.001, \phi=.69$; Handlungsorientierung: $\mathrm{SC}^{(+)}=8$ [89\%] vs. $\mathrm{SC}^{(-)}=2[13 \%]$; $\left.\chi^{2}[1, N=24]=13.21, p<.001, \phi=.74\right)$. 
$0.87, n s)$ noch in der vorliegenden Replikationsstudie $\left(\chi^{2}[1, N=38]=1.33, p=.249, \phi=-.19\right)$ ein Unterschied zwischen Handlungsorientierung und Lageorientierung nachweisbar. ${ }^{16}$

\subsubsection{Diskussion}

Die vorliegende Untersuchung stellte einen direkten Replikationsversuch zu van Putten et al. (2010) dar. Die Autoren berichteten, dass lageorientierte Personen mit höherer Wahrscheinlichkeit dem Sunk-Cost-Effekt unterliegen als handlungsorientierte Personen. Obwohl versucht wurde, die Originalstudie so genau wie möglich zu rekonstruieren, schlug die Replikation des zentralen Interaktionseffekts zwischen Handlungs- und Lageorientierung und den versunkenen Kosten fehl. Gleichzeitig konnten in der Replikationsstudie signifikante Sunk-Cost-Effekte auf beiden Persönlichkeitsstufen nachgewiesen werden, was in der Originalstudie nur für Lageorientierung der Fall war. Die Feststellung von van Putten et al. (2010), dass handlungsorientierte Personen ihre Investitionsentscheidung weitgehend unabhängig von versunkenen Kosten treffen, wurde also nicht bestätigt.

Unklar bleibt, in wie fern die negativen $B$-Regressionskoeffizienten, die in der Originalarbeit für die Simple-Slope-Analysen sowie den Sunk-Cost-Haupteffekt ausgewiesen wurden, zutreffend sind (vgl. Abschnitt 2.2.2.1). Der von den Autoren inhaltlich diskutierte Sunk-Cost-Effekt in Form einer gesteigerten Investitionsneigung würde eigentlich positive Regressionskoeffizienten voraussetzen. Letzteres war aber nur in der vorliegenden Replikationsstudie der Fall, deren Befunde also zweifelsfrei im Einklang mit Arkes und Blumers (1985) grundlegenden Befund stehen und auch in die von van Putten et al. (2010) selbst diskutierte Richtung weisen. Sollte es sich in den Berichten der Originalstudie nicht um

\footnotetext{
${ }^{16}$ Auch bei einer Berechnung mit dem Originalmedian (= 11) änderten sich die Ergebnismuster nicht. Über die Ausführungen des Originalartikels hinausgehend sei an dieser Stelle einerseits erwähnt, dass die Variablen Alter, Geschlecht, Studiengang und Semesterzahl keinen Einfluss auf die zentrale Befundlage hatten und anderseits keinerlei Ausschlüsse im Rahmen der berichteten Analysen vorgenommen werden mussten.
} 
einen Vorzeichenfehler handeln, so hätte die vorliegende Replikationsstudie diesbezüglich genau gegenteilige Ergebnisse erbracht.

Auf Basis der vorliegenden Untersuchungsergebnisse erscheint zunächst fraglich, ob es sich bei Handlungs- und Lageorientierung tatsächlich um eine Moderatorvariable für den Sunk-Cost-Effekt handelt. Es wären weitere Replikationsstudien notwendig, um die Replizierbarkeit der Originalbefunde von van Putten et al. (2010) entweder mit höherer Aussagekraft sicherzustellen oder zu falsifizieren. Darum wird an dieser Stelle auf ein abschließendes Urteil verzichtet und stattdessen auf die Kapitel 5 berichtete integrative Replikationsstudie verwiesen, welche einen zweiten Replikationsversuch auf Trait-Ebene darstellt. 


\subsection{Optimismus und Pessimismus}

In Abschnitt 2.2.2.2 wurde die Arbeit von Juliusson (2006) vorgestellt. Dieser berichtete einen moderierenden Einfluss von zustandsbezogenem Optimismus und Pessimismus auf den Sunk-Cost-Effekt. In der entsprechenden Untersuchung führte Optimismus zu einer gesteigerten Investitionsneigung, während Pessimismus zu einer gesteigerten Abbruchneigung in den verwendeten Sunk-Cost-Szenarien führte. Hohe versunkene Kosten verstärkten beide Neigungen jeweils im Vergleich zu niedrigen versunkenen Kosten.

Wie in Kapitel 3 erläutert, sollen im Rahmen der vorliegenden Arbeit für alle Trait- und State-Persönlichkeitsvariablen mindestens zwei Replikationsversuche durchgeführt werden. Hierdurch lassen sich genauere Aussagen über die Belastbarkeit der Effekte treffen. Daher werden in diesem Unterkapitel zwei direkte Replikationsstudien für Optimismus und Pessimismus auf State-Ebene in separaten Abschnitten berichtet - im Gegensatz zu der Handlungs- und Lageorientierung und dem Rational Thinking Style, bei denen die integrative Replikationsstudie den zweiten Replikationsversuch auf Trait-Ebene darstellt (vgl. Kapitel 5).

Bei der ersten direkten Replikationsstudie zu Optimismus und Pessimismus handelte es sich um eine Fragebogenstudie im Labor, bei der zweiten um eine Online-Untersuchung. Analog zu Juliusson (2006) wurde eine von den Hauptstudien abgekoppelte Studie zur Manipulationskontrolle durchgeführt, die im nun Folgenden als erstes vorgestellt wird.

\subsubsection{Studie zur Manipulationskontrolle}

\subsubsection{Methode}

\subsection{Stichprobe und Design}

Um die Wirksamkeit seiner probabilistischen Manipulation zu testen, führte Juliusson (2006) eine separate Untersuchung mit 48 Probanden durch. Im Rahmen der Replikationsstudie wurde eine vergleichbare Stichprobe gewählt, die insgesamt 49 
Studierende im Bachelorstudium an der Georg-August-Universität Göttingen umfasste. Davon waren 41 Probanden weiblich (84\%) und 8 männlich (16\%). Das Durchschnittsalter betrug 21,10 Jahre $(S D=1,65)$, in einer Spanne zwischen 18 und 26 Jahren. Wie in der Originalstudie wurde dabei ein 2 (hohe vs. niedrige versunkene Kosten) $\times 2$ (Optimismus vs. Pessimismus) - Zwischensubjekt-Design realisiert.

\subsection{Durchführung und Material}

Die Akquise erfolgte in drei verschiedenen Bachelor-Seminaren, deren Teilnehmer im Anschluss an die jeweilige Lehreinheit gefragt wurden, ob sie noch für eine kurze Fragebogenstudie bleiben möchten. Von der Versuchsleitung erhielten die Interessenten mündliche Informationen über den Ablauf der Befragung und die Entlohnung in Form eines Schokoladenriegels. Für alle Freiwilligen fand die Erhebung daraufhin im selben Raum wie das Seminar zuvor statt. Beim Verteilen der Fragebögen, die zuvor in zufälliger Reihenfolge durchmischt worden waren, wurden die Probanden randomisiert auf die vier Experimentalbedingungen aufgeteilt.

Wie in der Originalstudie wurde ein aus der Hauptuntersuchung entnommenes Szenario vorgelegt, in dem eine Fortsetzungsentscheidung über die Entwicklung eines neuen Produkts getroffen werden soll. Zur Manipulation der versunkenen Kosten sind dabei entweder hohe oder niedrige versunkene Kosten für einen bereits zurückliegenden Investitionszeitpunkt ausgewiesen. Für die Manipulation von Optimismus und Pessimismus wird die Gewinnwahrscheinlichkeit im initialen Investitionszeitpunkt variiert und beträgt entweder $20 \%$ auf Stufe des Optimismus oder 80\% auf Stufe des Pessimismus. Den Probanden wird mitgeteilt, dass sich die Gewinnwahrscheinlichkeit im Projektverlauf geändert habe und im Zeitpunkt der gegenwärtigen Investitionsentscheidung exakt 50\% beträgt. Durch Suggestion eines steigenden oder fallenden Trends, der sich ergibt, wenn man die Gewinnwahrscheinlichkeiten beider Zeitpunkte miteinander vergleicht, sollte der optimistische bzw. pessimistische Zustand erzeugt werden. 
Anstatt eine Investitionsentscheidung zu treffen, wurden die Probanden im Rahmen der Manipulationskontrolle gebeten, das beschriebene Investment auf drei neunstufigen Antwortskalen zu bewerten. Die drei Skalen waren definiert durch ihre Endpunkte: „schlechte Gelegenheit vs. gute Gelegenheit“, „pessimistisch vs. optimistisch“ und „nicht erfolgversprechend vs. erfolgversprechend“. Abschließend wurden auch das Geschlecht, das Alter und das Studienfach abgefragt. Nach der Bearbeitung wurden die Probanden von der Versuchsleitung über die Hintergründe dieser Untersuchung aufgeklärt, alle Fragebögen eingesammelt und jedem Teilnehmer im Gegenzug ein Schokoladenriegel ausgehändigt. Mit Dank und der Bitte um Verschwiegenheit wurden die Teilnehmer schließlich verabschiedet.

\subsubsection{Ergebnisse}

Wie in der Originalstudie fanden sich auch in der vorliegenden Replikationsstudie vergleichbar hohe Korrelationen unter den Skalen (gute Gelegenheit - optimistisch: $r_{\mathrm{s}}=.714$ [Original $r_{\mathrm{s}}=.664$ ]; gute Gelegenheit - erfolgversprechend: $r_{\mathrm{s}}=.721$ [Original $r_{\mathrm{s}}=.692$ ]; optimistisch - erfolgversprechend: $r_{\mathrm{s}}=.754$ [Original $r_{\mathrm{s}}=.818$ ]). Die höchste Reliabilität fand sich wie in der Originalstudie für eine Mittelung der Skalen „optimistisch vs. pessimistisch“ und „erfolgversprechend vs. nicht erfolgversprechend“, mit einem Cronbach’s Alpha Wert von .875 (Original $=.895){ }^{17}$

Wie in der Originalstudie wurden zwei 2 (ansteigender vs. fallender Trend) $\times 2$ (hohe vs. niedrige versunkene Kosten) - Varianzanalysen durchgeführt. In der ersten Analyse stellte die gemittelte Variable der beiden hoch korrelierten Items „optimistisch vs. pessimistisch“ und „erfolgversprechend vs. nicht erfolgversprechend“ die abhängige Variable dar. Hierbei zeigte sich ein signifikanter Haupteffekt für den ansteigenden vs. fallenden Trend $(M=6.6$ vs. 5.6, $F[1,45]=5.92, p=.019, \eta_{\mathrm{p}}^{2}=.12$; Original: $M=5.4$ vs. $\left.4.0, F[1,44]=4.35, p=.043\right)$.

\footnotetext{
${ }^{17}$ Die Cronbach's Alpha Werte der anderen Skalen wurden in der Originalstudie nicht berichtet. Die Werte in der vorliegenden Replikationsstudie lauteten wie folgt: „optimistisch vs. pessimistisch“ und „schlechte Gelegenheit vs. gute Gelegenheit“ $=.852$; ,schlechte Gelegenheit vs. gute Gelegenheit“ und ,erfolgversprechend vs. nicht erfolgversprechend“ $=.870$.
} 
In der zweiten Varianzanalyse, in der alleine „optimistisch vs. pessimistisch“ als abhängige Variable verwendet wurde, zeigte sich ebenfalls ein signifikanter Haupteffekt für den Trend $\left(M=6.8\right.$ vs. $5.7, F[1,45]=6.75, p=.013, \eta_{\mathrm{p}}^{2}=.13$; Original: $M=5.4$ vs. $4.0, F[1,44]=$ $5.16, p=.028)$.

\subsubsection{Diskussion}

Die Befunde der durchgeführten Manipulationskontrolle konnten jene aus der Originalarbeit durchgehend replizieren. Auch in der vorliegenden Untersuchung nahmen die Probanden bei einem ansteigenden Trend (induzierter Optimismus) ein potenzielles Investment optimistischer und erfolgversprechender wahr als bei einem fallenden Trend (induzierter Pessimismus). Infolge dessen kann davon ausgegangen werden, dass die Induktion von zustandsbezogenem Optimismus und Pessimismus, durch die probabilistische Manipulation des Gewinnwahrscheinlichkeitstrends in der beschriebenen Art und Weise, zuverlässig funktioniert und das Verfahren für die Anwendung in den folgenden direkten Replikationsstudien geeignet ist.

\subsubsection{Erste Replikationsstudie}

Die erste von zwei direkten Replikationsstudien auf State-Ebene wurde wie bei Juliusson (2006) als Fragebogenstudie, im Anschluss an eine Bachelor-Vorlesung an der Georg-AugustUniversität Göttingen, durchgeführt.

\subsubsection{Methode}

\subsection{Stichprobe und Design}

Um die Belastbarkeit der Befunde der Replikationsstudie zu erhöhen, wurde der Stichprobenumfang im Vergleich zur Originalstudie deutlich vergrößert (97 Probanden statt 52). Wie eingangs beschrieben, ist hierdurch näherungsweise von einer höheren Teststärke der Replikationsstudie auszugehen. Das Durchschnittsalter der 64 weiblichen (66\%) und 33 
männlichen (34\%) Probanden betrug 21,93 Jahre $(S D=3,27)$, in einer Spanne zwischen 19 und 41 Jahren.

Wie in der Originalstudie wurde ein 2 (hohe vs. niedrige versunkene Kosten) $\times 2$ (exakte vs. ungefähre Gewinnwahrscheinlichkeit) $\times 2$ (induzierter Optimismus vs. Pessimismus) Design realisiert. Die versunkenen Kosten und die Gewinnwahrscheinlichkeit wurden dabei jeweils als Innersubjektfaktoren operationalisiert und Optimismus vs. Pessimismus als Zwischensubjektfaktor. Entsprechend der Arbeit von Juliusson (2006) dienten zum einen die dichotome Investitionsentscheidung und zum anderen ein metrischer Präferenzindex als abhängige Variablen, für deren Messung die gleichen Sunk-Cost-Szenarien zum Einsatz kamen wie in der Originalstudie.

\subsection{Durchführung und Material}

Analog zur Originalstudie wurden die potenziellen Teilnehmer im Anschluss an eine Vorlesung gefragt, ob sie für eine kurze Fragebogenstudie zum Thema „Entscheidungsverhalten“ im Hörsaal bleiben würden. Die Versuchsleitung informierte dabei auch über den Ablauf der Erhebung und die Vergütung. Bei letzterem handelte es sich wahlweise um 3 Euro oder eine halbe Versuchspersonenstunde zur Anrechnung im Psychologie-Bachelorstudium. Zusätzlich erhielt jeder Teilnehmer einen Schokoladenriegel. Die Erhebung fand mit allen Probanden gleichzeitig im Hörsaal der vorherigen Vorlesung statt. Die Probanden bekamen von der Versuchsleitung einen Fragebogen ausgehändigt, mit dessen Bearbeitung sie direkt nach dem Austeilen begannen.

Die Fragebögen orientierten sich inhaltlich so nahe wie möglich an den Ausführungen von Juliusson (2006), dessen Originalmaterial leider nicht mehr zugänglich war. Die verwendeten Fragebögen umfassten ebenfalls fünf Seiten: Auf der ersten Seite wurden die Probanden gebeten, ihr Geschlecht, Alter und Studienfach anzugeben. Außerdem erhielten sie allgemeine schriftliche Instruktionen über die Bearbeitung, Vergütung und Anonymität. Dabei wurde wie in der Originalstudie darauf hingewiesen, dass die vier auf den nächsten Seiten folgenden 
Szenarien trotz ihrer Strukturgleichheit sorgfältig durchgelesen und unabhängig voneinander bearbeitet werden sollen. Es waren deshalb vier Szenarien, da hierin die beiden zweistufigen Innersubjektfaktoren (versunkene Kosten \& Gewinnwahrscheinlichkeit) durch eine Variation der zugehörigen quantitativen Parameter manipuliert wurden (siehe Anhang A). Um Reihenfolgeeffekte zu vermeiden, wurden die vier Szenarien bereits im Zuge der Fragebogenerstellung in unterschiedlichen Reihenfolgen angeordnet. Die Manipulation von Optimismus und Pessimismus erfolgte inter-individuell durch zwei verschiedene Fragebogenversionen. Die Aufteilung der Probanden auf beide Faktorstufen erfolgte randomisiert im Zuge der Fragebogenverteilung.

In den von Juliusson (2006) übernommen Szenarien wird der Proband gebeten sich vorzustellen, er habe in die Entwicklung eines neuen Produkts investiert. Von diesem Produkt wird erwartet, dass es wettbewerbsfähig am Markt sein würde und dementsprechend Gewinne generieren kann. Die Wahrscheinlichkeit für Gewinne betrug zum Zeitpunkt der initialen Investition entweder 20\% (Optimismus) oder 80\% (Pessimismus). Um das Projekt zu verwirklichen, stand ein begrenztes Budget in Höhe von 10.000 Euro zur Verfügung, wovon bereits entweder 5000 Euro $\left(\mathrm{SC}^{(+)}\right)$oder 1700 Euro $\left(\mathrm{SC}^{(-)}\right)$investiert wurden. Im gegenwärtigen Zeitpunkt erfährt der Proband, dass die Entwicklung des Produkts zwar erwartungsgemäß verlaufen sei, jedoch die Kosten unterschätzt wurden. Es sind nun weitere 5000 Euro notwendigerweise zu investieren, um die Entwicklung abschließen zu können. ${ }^{18}$ Der Proband muss sich an dieser Stelle entscheiden, ob er den Betrag investieren oder durch eine Nicht-Investition das Projekt abbrechen möchte. Es wird der Hinweis gegeben, dass die erste Investition im Falle eines Abbruchs verloren geht. Ferner wird darauf hingewiesen, dass mit dem Produkt später Gewinne erzielbar sind, die die gesamten Investitionskosten

\footnotetext{
${ }^{18}$ In der schwedischen Originalstudie wurden 60.000 SEK für das Budget, 30.000 SEK als versunkene Kosten in der $\mathrm{SC}^{(+)}$-Version und 10.000 $\mathrm{SEK}$ als versunkene Kosten in der $\mathrm{SC}^{(-)}$-Version verwendet. Die Reinvestitionshöhe betrug 30.000 SEK. Um den Probanden in der vorliegenden Replikationsstudie die Bearbeitung zu erleichtern, wurden stattdessen vergleichbare Eurobeträge verwendet.
} 
übersteigen. Im gleichen Zuge erfolgt der für die Manipulation von Optimismus und Pessimismus entscheidende Hinweis, dass die Gewinnwahrscheinlichkeit inzwischen revidiert wurde und nun bei 50\% (exakte Gewinnwahrscheinlichkeit) oder zwischen $30 \%$ und $70 \%$ (ungefähre Gewinnwahrscheinlichkeit) liegt. Die Manipulation von Optimismus und Pessimismus ergibt sich also durch Suggestion eines ansteigenden bzw. abnehmenden Trends der Gewinnwahrscheinlichkeit, wie bereits im Rahmen der separaten Studie zur Manipulationskontrolle beschrieben (vgl. Abschnitt 4.2.1). Um den Probanden die Bearbeitung zu erleichtern, waren die variierten Zahlenwerte der verwendeten Faktoren in den ansonsten inhaltlich übereinstimmenden Szenarien jeweils fett formatiert. Ohne diese Formatierung wurde im Vorfeld die Gefahr als zu hoch eingeschätzt, dass die relevanten Informationen zu leicht übersehen werden könnten.

Nach der vollständigen Bearbeitung, gaben die Probanden ihre Fragebögen selbständig bei der Versuchsleitung wieder ab. ${ }^{19}$ Im Gegenzug erhielten sie ihre Probandenvergütung und einen Handzettel mit einer schriftlichen Aufklärung über die Hintergründe der Untersuchung. Mit Dank wurden die Probanden von der Versuchsleitung nach und nach verabschiedet.

\subsubsection{Ergebnisse}

Die Investitionsentscheidung wurde durch Ankreuzen der gewünschten dichotomen Antwortoptionen ,investieren“ oder „abbrechen“ erhoben. Außerdem sollten die Probanden auf einer Skala zwischen 0 (neutral) und 10 (voll und ganz) ankreuzen, wie sehr sie zu dieser Entscheidung stehen. Wie bei Juliusson (2006) wurden zwei mehrfaktorielle Varianzanalysen (2 [induzierter Optimismus vs. Pessimismus] x 2 [niedrige vs. hohe versunkene Kosten] x 2 [exakte vs. ungefähre Gewinnwahrscheinlichkeit] - ANOVA) mit Messwiederholung über die letzten zwei Faktoren durchgeführt. Hierbei wurde für die Analyse der kategorialen

\footnotetext{
19 Juliussion (2006) berichtete eine ungefähre Bearbeitungsdauer des Fragebogens von 10 Minuten, ohne Angabe, ob es sich hierbei um eine Schätzung oder eine systematische Messung handelte. In der Replikationsstudie wurde aufgrund der beschriebenen Durchführungsbedingungen keine systematische Erhebung der Bearbeitungszeit durchgeführt.
} 
abhängigen Variable die Codierung 0 für „,abbrechen“ und 1 für „,investieren“ gewählt. Die Basis für die Berechnung des „Präferenzindex“ als zweite abhängige Variable bildeten die Angaben zwischen 0 und 10, denen entweder ein positives oder negatives Vorzeichen zugewiesen wurde, je nachdem ob die Probanden zuvor die Entscheidung ,investieren“ (positiv) oder „abbrechen“ (negativ) gewählt hatten. Es konnten sich also Präferenzindizes in einer Spanne zwischen -10 und +10 ergeben, wobei der Wert 0 eine Indifferenz zwischen beiden Handlungsoptionen repräsentiert.

Für eine bestmögliche Vergleichbarkeit der Analysebefunde werden die Ergebnisse aus Juliussons (2006) Originalstudie in Tabelle 4 aufgelistet:

Effects of a 2 by 2 by 2 ANOVA on percentage of choices to continue

\begin{tabular}{ll}
\hline Induced Optimism vs. Pessimism & $F(1,44)=29.32, p<.001$ \\
Sunk Cost $*$ Optimism vs. Pessimism & $F(1,44)=5.43, p=.024$ \\
\hline $\begin{array}{l}\text { Sunk Cost (for Optimism in a paired samples } t \text {-test) } \\
\text { Effects of a } 2 \text { by } 2 \text { by } 2 \text { ANOVA on preference ratings }\end{array}$ \\
\hline $\begin{array}{l}\text { Induced Optimism vs. Pessimism } \\
\text { Sunk Cost } * \text { Optimism vs. Pessimism }\end{array}$ & $F(1,44)=27.44, p<.001$ \\
\hline Sunk Cost (for Optimism in a paired samples t-test) & $F(1,44)=3.37, p=.073$ \\
\hline
\end{tabular}

Tabelle 4: Ergebnisse der Varianzanalysen von Juliusson (2006) mit der dichotomen Investitionsentscheidung und dem Präferenzindex als abhängige Variablen.

In der Analyse der dichotomen Investitionsentscheidung zeigte sich bei Juliusson (2006) die zentrale Interaktion zwischen den versunkenen Kosten und Optimismus vs. Pessimismus. Dabei war sowohl die Fortführungstendenz der Optimisten, als auch die Abbruchtendenz der Pessimisten umso stärker ausgeprägt, wenn hohe gegenüber niedrigen versunkenen Kosten vorlagen. In der entsprechenden Analyse der Replikationsstudie konnte diese Interaktion jedoch nicht repliziert werden $\left(F[1,95]=.55, p=.460, \eta_{\mathrm{p}}^{2}=.01\right)$. Gleiches gilt für die parallele Analyse mit dem Präferenzindex als abhängige Variable $(F[1,95]=.65, p=.421$, $\left.\eta_{\mathrm{p}}{ }^{2}=.01\right)$. Die Ergebnisse der Replikationsstudie können eine moderierende Wirkung des zustandsbezogenen Optimismus und Pessimismus auf den Sunk-Cost-Effekt somit nicht bestätigen. Darüber hinaus fand sich auch deskriptiv nicht das beschriebene 
Interaktionsmuster aus der Originalstudie. Tendenziell kehrte sich die Interaktion sogar um, da Pessimisten eine erhöhte und Optimisten eine verminderte Fortführungstendenz aufwiesen, wenn hohe gegenüber niedrigen versunkenen Kosten vorlagen (vgl. Tabelle 6).

Wie in der Originalstudie wurden separate $t$-Tests bei gepaarten Stichproben für beide Persönlichkeitsstufen berechnet, um Sunk-Cost-Effekte nachzuweisen. In der Originalstudie wurde dabei auf der Stufe von Optimismus ein signifikanter Sunk-Cost-Haupteffekt für beide abhängigen Variablen berichtet. In der Replikationsstudie konnte hingegen auf keiner der beiden Stufen ein Haupteffekt für die versunkenen Kosten nachgewiesen werden, weder in der Analyse der dichotomen abhängigen Variablen (Pessimismus: $\left[M_{(S C+)}=66,3 \%\right.$ vs. $M_{\left(S C_{-}\right)}=$ 61,2\%], $t[48]=.73, p=.471$; Optimismus: $\left[M_{(S C+)}=81,3 \%\right.$ vs. $\left.M_{(S C-)}=83,3 \%\right], \mathrm{t}[47]=-.31$, $p=.755)$, noch in der Analyse des Präferenzindex (Pessimismus: $\left[M_{(S C+)}=2,0\right.$ vs. $M_{(S C-)}=$ $1,7], t[48]=.38, p=.703$; Optimismus: $\left[M_{(S C+)}=4,5\right.$ vs. $\left.\left.M_{(S C-)}=5.1\right], t[47]=-.80, p=.429\right)$.

Abseits der zentralen Analysen konnte nur der signifikante Haupteffekt für Optimismus vs. Pessimismus von Juliusson (2006) repliziert werden. Demnach zeigten sich die Optimisten insgesamt investitionsfreudiger als die Pessimisten, sowohl in der Analyse der dichotomen Investitionsentscheidung $\left(F[1,95]=9.87, p=.002, \eta_{\mathrm{p}}{ }^{2}=.09\right)$ als auch in der Analyse des Präferenzindex $\left(F[1,95]=11.48, p=.001, \eta_{\mathrm{p}}{ }^{2}=.11\right)$. Dieses Ergebnis stellt allerdings mehr oder weniger eine erweiterte Konstruktvalidierung dar und steht in keinem Zusammenhang zur eigentlichen Fragestellung. ${ }^{20}$

\footnotetext{
${ }^{20}$ Eine Interaktion zwischen Optimismus vs. Pessimismus und der Gewinnwahrscheinlichkeit zeigte sich wie in der Originalstudie nicht (Investitionsentscheidung: $F[1,95]=1.58, p=.212, \eta \mathrm{p}^{2}=.02$; Präferenzindex: $\left.F[1,95]=1.88, p=.173, \eta p^{2}=.02\right)$. Einen marginalen Interaktionseffekt im Rahmen der Analyse der Investitionsentscheidung führte Juliusson (2006) auf die Pessimismusbedingungen zurück ( $M=31.5 \%$ vs. $21.1 \% ; t[25]=1.49, p=.147$ ), zwischen denen sich, wie in der Originalstudie, auch in der Replikationsstudie kein signifikanter Unterschied hinsichtlich der Gewinnwahrscheinlichkeit fand $(M=61.2 \%$ vs. $66.3 \% ; t[48]=-$ $.90, p=.375)$. Hinsichtlich der zentralen Fragestellung sind diese Nebenbefunde allerdings nicht relevant. Bei einem Ausschluss aller wirtschaftswissenschaftlichen Studenten, die in der Originalstudie nicht befragt wurden, änderte sich nichts an der zentralen Befundlage. Eine über die Originalstudie hinausgehende Analyse mit Alter, Geschlecht und Studiengang als Kontrollvariablen, erbrachte zwar signifikante Sunk-Cost-Haupteffekte für beide abhängigen Variablen $\left(F \mathrm{~s}[1,92]>4.60, p \mathrm{~s}<.035, \eta p^{2} \mathrm{~s}>.048\right)$, jedoch nicht die entscheidende Interaktion.
} 
Zur Veranschaulichung werden in den Tabellen 5 und 6 die deskriptiven Ergebnismuster beider Studien direkt gegenübergestellt. Hierin sind jeweils die mittleren Präferenzindizes $(M)$, Standardabweichungen $(S D)$ und prozentualen Häufigkeiten der dichotomen Entscheidung in den Spalten aufgelistet. Die Unterteilung in den Zeilen erfolgt nach den Versuchsbedingungen. Höhere Werte als 50\% stehen in den Tabellen für eine überwiegende Entscheidung zur Reinvestition, geringere Werte für eine überwiegende Entscheidung zum Abbruch. $^{21}$

Tabelle 5: Deskriptive Ergebnisse von Juliusson (2006): Prozentuale Investitionshäufigkeiten und Präferenzindizes in den Versuchsbedingungen, aufgeteilt nach den Stufen des induzierten Optimismus vs. Pessimismus, der versunkenen Kosten und der Gewinnwahrscheinlichkeit.

\begin{tabular}{|c|c|c|c|c|c|c|}
\hline & \multicolumn{3}{|c|}{ Induced pessimism } & \multicolumn{3}{|c|}{ Induced optimism } \\
\hline & $M$ & $(S D)$ & $\%$ & $M$ & $(S D)$ & $\%$ \\
\hline \multicolumn{7}{|l|}{ Low sunk cost } \\
\hline Exact probability & -2.4 & (5.5) & 39.9 & 1.6 & $(6.2)$ & 62.9 \\
\hline Ambiguous probability & -4.0 & (4.9) & 19.6 & 2.1 & $(6.0)$ & 65.0 \\
\hline \multicolumn{7}{|l|}{ High sunk cost } \\
\hline Exact probability & -3.2 & $(5.2)$ & 23.2 & 4.0 & (5.7) & 77.1 \\
\hline Ambiguous probability & -3.5 & $(5.0)$ & 22.6 & 3.9 & $(4.8)$ & 85.7 \\
\hline
\end{tabular}

Tabelle 6: Deskriptive Ergebnisse der ersten Replikationsstudie zu Juliusson (2006): Prozentuale Investitionshäufigkeiten und Präferenzindizes in den Versuchsbedingungen, aufgeteilt nach den Stufen des induzierten Optimismus vs. Pessimismus, der versunkenen Kosten und der Gewinnwahrscheinlichkeit.

\begin{tabular}{|c|c|c|c|c|c|c|}
\hline & \multicolumn{3}{|c|}{ Induced pessimism } & \multicolumn{3}{|c|}{ Induced optimism } \\
\hline & $M$ & $(S D)$ & $\%$ & $M$ & $(S D)$ & $\%$ \\
\hline \multicolumn{7}{|l|}{ Low sunk cost } \\
\hline Exact probability & 1.6 & $(7.5)$ & 59.2 & 5.7 & $(4.8)$ & 85.4 \\
\hline Ambiguous probability & 1.7 & $(7.1)$ & 63.3 & 4.5 & $(5.4)$ & 81.3 \\
\hline \multicolumn{7}{|l|}{ High sunk cost } \\
\hline Exact probability & 1.9 & $(6.6)$ & 63.3 & 5.0 & $(4.3)$ & 83.3 \\
\hline Ambiguous probability & 2.1 & $(6.0)$ & 69.4 & 3.9 & $(5.2)$ & 79.2 \\
\hline
\end{tabular}

${ }^{21}$ Die deskriptiven Ergebnisse zeigen, dass die Pessimisten nicht wie bei Juliusson (2006) überwiegend den Abbruch favorisierten, sondern überwiegend zur Projektfortsetzung tendierten, wenn auch im schwächeren Umfang als die Optimisten. Ebenfalls konträr zur Originalstudie tendierten Optimisten bei einer exakten Gewinnwahrscheinlichkeit eher zur Projektfortsetzung als bei einer ungefähren Gewinnwahrscheinlichkeit. Das Gegenteil war für Pessimisten der Fall. Diese Ergebnisse können zwar ebenfalls das Muster der Originalstudie nicht replizieren, sind im Hinblick auf die zentrale Fragestellung jedoch nicht weiter relevant. 


\subsubsection{Diskussion}

Bei der vorliegenden Untersuchung handelte es sich um die erste von zwei direkten Replikationsstudien zu Juliusson (2006). Dieser berichtete einen moderierenden Einfluss von zustandsbezogenem Optimismus und Pessimismus auf den Sunk-Cost-Effekt. Optimismus führte in seiner Untersuchung zu einer gesteigerten Investitionsneigung und Pessimismus zu einer gesteigerten Abbruchneigung, während hohe gegenüber niedrigen versunkenen Kosten diese Neigungen jeweils noch weiter verstärkten.

Im Sinne einer exakten Replikation wurde versucht, alle Aspekte der Originalstudie so genau wie möglich nachzustellen. Außerdem wurde der Stichprobenumfang nahezu verdoppelt, um eine höhere Teststärke als die Originalstudie zu erreichen. Dennoch war eine Interaktion im obigen Sinne nicht nachweisbar. Stattdessen ging die Investitionstendenz der Probanden im Vergleich zur Originalstudie deskriptiv sogar in die gegenteilige Richtung, denn die Investitionsneigung der Optimisten nahm bei hohen versunkenen Kosten tendenziell ab, bei Pessimisten hingegen zu. Auf Basis der vorliegenden Ergebnisse wird daher nicht nur die grundsätzliche moderierende Wirkung von zustandsbezogenem Optimismus und Pessimismus auf den Sunk-Cost-Effekt infrage gestellt, sondern auch in welche Richtung eine mögliche Interaktion überhaupt wirkt.

Auch der Sunk-Cost-Effekt auf Stufe von Optimismus, wie er bei Juliusson (2006) berichtet wurde, konnte nicht repliziert werden. Abseits der zentralen Analysen bestätigte sich lediglich der generelle Haupteffekt für Optimismus vs. Pessimismus aus der Originalstudie, wonach Optimisten eine höhere Investitionsneigung zeigten als Pessimisten. Dieser Befund ist hinsichtlich der zentralen Fragestellung allerdings nicht relevant.

Im folgenden Abschnitt wird wie geplant noch die zweite direkte Replikationsstudie berichtet, weshalb an dieser Stelle zunächst kein abschließendes Urteil gefällt werden soll. 


\subsubsection{Zweite Replikationsstudie}

Auch die zweite direkte Replikationsstudie orientierte sich so nahe wie möglich an der Originalstudie von Juliusson (2006). Daher wurden alle Inhalte der ersten Replikationsstudie wiederholt, abgesehen von der Tatsache, dass diese Untersuchung nun als Onlinestudie realisiert wurde.

\subsubsection{Methode}

\subsection{Stichprobe und Design}

Bei den Teilnehmern handelte es sich auch in dieser Untersuchung um Studierende der Georg-August-Universität Göttingen. Wie in der ersten Replikationsstudie wurde der Stichprobenumfang im Vergleich zur Originalstudie größer gewählt (102 Probanden statt 52), um eine höhere Teststärke zu erreichen. Davon waren 64 Teilnehmer weiblich (63\%) und 38 männlich (37\%). Das Durchschnittsalter betrug 21,78 Jahre $(S D=4,30)$, in einer Spanne zwischen 17 und 43 Jahren. Da es im Rahmen von Onlineerhebungen zu selbständigen Abbrüchen der Teilnehmer kommen kann, wurden nur Datensätze verwendet, in denen alle Sunk-Cost-Szenarien vollständig bearbeitet wurden. Aufgrund von Mehrfachteilnahmen wurden drei Datensätze von den Analysen ausgeschlossen und nur die jeweils erste Teilnahme gewertet.

Es wurde das gleiche $2 \times 2 \times 2$ Design realisiert wie zuvor, mit den versunkenen Kosten und der Gewinnwahrscheinlichkeit als Innersubjektfaktoren sowie Optimismus vs. Pessimismus als Zwischensubjektfaktor. Die dichotome Investitionsentscheidung sowie der metrische Präferenzindex stellten wiederum die abhängigen Variablen dar und wurden mithilfe der bereits vorgestellten Sunk-Cost-Szenarien von Juliusson (2006) erhoben.

\subsection{Durchführung und Material}

Im Vergleich zur ersten Replikationsstudie erfolgten Anpassungen an die nun vollzogene Form der Onlineerhebung. So nahmen die Teilnehmer hinsichtlich ihrer Probandenvergütung 
entweder an einer Verlosung von insgesamt zehn mal 20 Euro teil oder erhielten eine halbe Versuchspersonenstunde für die Anrechnung in ihrem Psychologie-Bachelorstudium. Die Akquise erfolgte über studentische Onlineplattformen, in denen Interessenten eine Verlinkung zu der vorliegenden Befragung mit dem Thema „Produktentwicklung“ fanden. Als Erhebungsplattform wurde „LimeSurvey“ verwendet (http://www.limesurvey.org). Diese bot zum Erhebungszeitpunkt allerdings nicht die Möglichkeit, verschiedene Versuchsbedingungen unter derselben Fragebogenadresse zu definieren. Darum wurden die Bedingungen in Form von Einzelfragebögen mit eigenen Adressen realisiert. Für Optimismus sowie Pessimismus existierten verschiedene Fragebogenversionen, innerhalb derer die Reihenfolge der Sunk-Cost-Szenarien wieder variiert wurde, um Reihenfolgeeffekten vorzubeugen. Insgesamt ergaben sich hierdurch acht Fragebogenversionen, auf die die Teilnehmer zufällig verteilt wurden, indem beim Öffnen des kommunizierten Hauptlinks zunächst ein Skript zwischengeschaltet wurde, das die Probanden randomisiert zu einem der Fragebögen weiterleitete.

Auf der ersten Fragebogenseite erfolgte eine schriftliche Begrüßung der Probanden. In diesem Textabschnitt erhielten sie, wie in der Untersuchung zuvor, allgemeine Informationen über die Bearbeitung, Vergütung und Anonymität. Zur nächsten Seite gelangten die Probanden indem sie auf „Weiter“ klickten.

Auf der zweiten Seite wurden die Probanden gebeten, Angaben zu ihrer Person zu machen. Bei der dichotomen Auswahlmöglichkeit für Geschlecht sowie den offenen Feldern für Alter und Studienfach handelte es sich jeweils um Pflichtangaben. Durch einen Klick auf „Weiter“ gelangten die Probanden zur nächsten Seite. Auf dieser wurde eine schriftliche Erklärung der Aufgabenstellung angezeigt, in der wieder darauf hingewiesen wurde, dass die vier folgenden Szenarien trotz ihrer Strukturgleichheit sorgfältig durchgelesen und unabhängig voneinander bearbeitet werden sollten. Wie in der ersten Replikationsstudie, waren die variierten Zahlenwerte in den ansonsten identischen Szenarien wieder fett 
formatiert, um den Probanden die Bearbeitung zu erleichtern. Durch einen Klick auf die Erklärung „Ich bin bereit““ startete die Bearbeitung der vier Sunk-Cost-Szenarien auf separaten Seiten, wie in der ersten Replikationsstudie beschrieben. Dabei trafen die Probanden ihre Entscheidung jeweils durch das Anklicken der vorgegebenen Antwortmöglichkeiten. Bei der Frage „Möchten Sie weitere $5000 €$ in das Projekt investieren?“ hießen die Auswahlmöglichkeiten ,ja“ und „nein“. Bei der Frage „Wie sehr stehen Sie zu dieser Entscheidung?“ lag dagegen eine elfstufige Auswahlmöglichkeit mit Werten zwischen 0 und 10 vor. In beiden Fällen handelte sich um Pflichtangaben.

Nachdem alle relevanten Daten wie in der Originalstudie abgefragt waren, folgte ergänzend ein Abschlussfragebogen. Darin wurde zuerst in einem offenen Textfeld abgefragt, ob schon einmal an einer ähnlichen Studie teilgenommen wurde. Falls dies zutraf, sollte beschrieben werden, wann diese ähnliche Studie stattfand und worum es darin ging. In einem zweiten offenen Textfeld sollte im Sinne einer Verdachtskontrolle niedergeschrieben werden, worum es nach Meinung der Probanden in der vorliegenden Untersuchung ging. Danach folgte eine Auflistung zur Abfrage der gewünschten Probandenvergütung. Hier musste einer der folgenden drei Punkte ausgewählt werden: a) „Verlosung“, b) „Halbe Versuchspersonenstunde“ oder c) „Keines von beidem“. Anschließend wurden die Probanden noch gebeten, ihre E-Mail-Adresse in einem offenen Textfeld angeben, wobei es sich um eine freiwillige Angabe handelte. Es wurde darauf hingewiesen, dass die Adressen nur der Kommunikation bezüglich der Versuchspersonenvergütung und der Aufklärung über die Hintergründe der Untersuchung dienten. Auf der letzten Seite des Onlinefragebogens wurde den Teilnehmern gedankt und noch einmal darauf hingewiesen, dass sie nach Abschluss der 
Erhebungen zu den beiden vorgenannten Punkten wieder kontaktiert werden. Der spätere EMail-Versand erfolgte per Blindkopie über separate Verteiler. ${ }^{22}$

\subsubsection{Ergebnisse}

Die folgenden Varianzanalysen und ihre Codierungen entsprechen denen der ersten Replikationsstudie. Für einen optimalen Vergleich zu den Ergebnissen der Originalstudie und denen der ersten Replikationsstudie, wird ein Rückblick auf die Tabellen aus dem vorherigen Abschnitt 4.2.2.2 empfohlen.

Im Fokus dieser zweiten Replikationsstudie stand wiederum die Replikation des Interaktionseffekts zwischen Optimismus vs. Pessimismus und den versunkenen Kosten, den Juliusson (2006) im Rahmen seiner Analyse der dichotomen Investitionsentscheidung berichtete. Allerdings konnte der Effekt auch in dieser zweiten Replikationsstudie nicht repliziert werden, weder für die dichotome Investitionsentscheidung $(F[1,100]=.60$, $\left.p=.442, \eta_{\mathrm{p}}^{2}=.01\right)$ noch für den Präferenzindex, $\left(F[1,100]=.07, p=.795, \eta_{\mathrm{p}}^{2}=.00\right)$. Eine moderierende Wirkung von zustandsbezogenem Optimismus und Pessimismus auf den SunkCost-Effekt erscheint daher weiterhin zweifelhaft.

Erneut fand sich auch in dieser Untersuchung das deskriptive Interaktionsmuster aus der Originalstudie nicht wieder (vgl. Tabelle 7). Zwar nahm die Investitionsneigung der Optimisten tendenziell bei hohen versunkenen Kosten in Übereinstimmung mit der Originalstudie zu, gleiches galt aber ebenso für die Pessimisten, die gemäß Juliusson (2006) eigentlich weniger hätten investieren müssen. Auf Basis der Ergebnisse aus der vorliegenden

\footnotetext{
${ }^{22}$ Wie erwähnt berichtete Juliussion (2006) eine ungefähre Bearbeitungsdauer des Fragebogens von 10 Minuten, wobei es sich möglicherweise nur um eine Schätzung handelte. In dieser onlinebasierten Replikationsstudie war eine systematische Messung hingegen zweifelsfrei möglich. Ein Vergleich der Startzeit mit der Beendigungszeit erbrachte eine durchschnittliche Bearbeitungsdauer von 6 Minuten. Der Grund für die kürzere Bearbeitungszeit könnte in der beschriebenen fetten Formatierung der variierten Zahlenwerte liegen, die den Probanden die Bearbeitung erleichtern sollte (siehe Anhang A). Sofern dieses bei Juliussion (2006) weniger übersichtlich gehandhabt wurde, was nicht nachprüfbar ist, da das Originalmaterial wie erwähnt nicht mehr zugänglich war, ließe sich die Zeitersparnis in der Replikationsstudie unter Umständen hierauf zurückführen.
} 
Replikationsstudie handelte es sich in der Tendenz also eher um einen generellen Effekt und nicht um die vorhergesagte Interaktion.

Sunk-Cost-Effekte waren in separaten $t$-Tests für beide Persönlichkeitsstufen auch in dieser Replikationsstudie nicht nachweisbar, weder für die dichotome Investitionsentscheidung (Pessimismus: $\left[M_{(S C+)}=65,1 \%\right.$ vs. $\left.M_{(S C-)}=62,3 \%\right], t[52]=.42$, $p=.679$; Optimismus: $\left[M_{(S C+)}=78,6 \%\right.$ vs. $\left.\left.M_{(S C-)}=68.4 \%\right], t[48]=1.53, p=.133\right)$ noch für den Präferenzindex (Pessimismus: $\left[M_{(S C+)}=1.7\right.$ vs. $\left.M_{\left(S C_{-}\right)}=1.5\right], t[52]=.25, p=.802$; Optimismus: $\left[M_{(S C+)}=3.8\right.$ vs. $\left.\left.M_{(S C-)}=3.2\right], t[48]=.67, p=.509\right)$. Bei Juliusson (2006) wurde zumindest für Optimismus ein signifikanter Sunk-Cost-Effekt berichtet. ${ }^{23}$

Abseits der zentralen Analysen zeigte sich in Übereinstimmung mit der Originalstudie wieder ein Haupteffekt für Optimismus vs. Pessimismus als Nebenbefund, jedoch nur für den Präferenzindex als abhängige Variable $\left(F[1,100]=4.27, p=.041, \eta_{\mathrm{p}}{ }^{2}=.04\right)$. Demnach war die Präferenz zur Fortführung des Projekts bei den Optimisten signifikant stärker ausgeprägt als bei den Pessimisten. Im Rahmen der Analyse der dichotomen Investitionsentscheidung blieb dieser Effekt aus $\left(F[1,100]=2.50, p=.117, \eta_{\mathrm{p}}{ }^{2}=.02\right) .{ }^{24}$

Entsprechend der Darstellung aus dem vorherigen Abschnitt, gibt Tabelle 7 einen Überblick über die deskriptiven Ergebnisse dieser zweiten Replikationsstudie.

\footnotetext{
${ }^{23}$ Aufgrund der Fragen im Abschlussfragebogen musste niemand von den Analysen ausgeschlossen werden. Drei Personen beschrieben im Suspicion Check inhaltlich zwar einen Escalation-Kontext im weiteren Sinne, jedoch ohne konkret den Sunk-Cost-Effekt zu benennen. Bei ihrem Ausschluss änderte sich in einer Kontrollrechnung nichts an den zentralen Befunden, lediglich der Nebenbefund eines Haupteffekts für Optimismus vs. Pessimismus zeigt sich dabei für die dichotome AV signifikant $\left(F[1,100]=4.37, p=.039, \eta p^{2}\right.$ $=.043)$. Darüber hinaus änderte sich weder bei einem Ausschluss aller wirtschaftswissenschaftlichen Studierenden noch unter Einbezug von Alter, Geschlecht und Studiengang als Kontrollvariablen etwas an der zentralen Befundlage. Abseits der zentralen Fragestellung zeigten sich in den Hauptanalysen noch signifikante Haupteffekte der Gewinnwahrscheinlichkeit für beide abhängigen Variablen $(F \mathrm{~s}>9.56, p \mathrm{~s}<.003)$.

${ }^{24}$ Bei einer Betrachtung der deskriptiven Ergebnisse fällt auf, dass auch in dieser Studie (und konträr zur Originalstudie) eine generell erhöhte Investitionsneigung vorlag, da neben den Optimisten auch die Pessimisten mehrheitlich zur Projektfortsetzung tendierten, wenn auch in schwächerem Umfang als die Optimisten.
} 
Tabelle 7: Deskriptive Ergebnisse der zweiten Replikationsstudie zu Juliusson (2006): Prozentuale Investitionshäufigkeiten und Präferenzindizes in den Versuchsbedingungen, aufgeteilt nach den Stufen des induzierten Optimismus vs. Pessimismus, der versunkenen Kosten und der Gewinnwahrscheinlichkeit.

\begin{tabular}{|c|c|c|c|c|c|c|}
\hline & \multicolumn{3}{|c|}{ Induced pessimism } & \multicolumn{3}{|c|}{ Induced optimism } \\
\hline & $M$ & $(S D)$ & $\%$ & $M$ & $(S D)$ & $\%$ \\
\hline \multicolumn{7}{|l|}{ Low sunk cost } \\
\hline Exact probability & 2.1 & $(7.2)$ & 66.0 & 4.3 & $(6.3)$ & 75.5 \\
\hline Ambiguous probability & 0.8 & (7.1) & 58.5 & 2.1 & $(7.3)$ & 61.2 \\
\hline \multicolumn{7}{|l|}{ High sunk cost } \\
\hline Exact probability & 2.9 & $(6.1)$ & 73.6 & 4.3 & $(6.2)$ & 81.6 \\
\hline Ambiguous probability & 0.5 & $(6.3)$ & 56.6 & 3.3 & $(6.6)$ & 75.5 \\
\hline
\end{tabular}

\subsubsection{Diskussion}

Die vorliegende Untersuchung stellte einen zweiten direkten Replikationsversuch zu Juliusson (2006) dar, der einen moderierenden Einfluss von zustandsbezogenem Optimismus und Pessimismus auf den Sunk-Cost-Effekt berichtete.

Allerdings konnte die zentrale Interaktion zwischen Optimismus vs. Pessimismus und den versunkenen Kosten wie schon in der ersten Replikationsstudie nicht repliziert werden. Auf Basis der Befundlage beider Replikationsstudien, die durch ihre vergrößerten Stichprobenumfänge jeweils eine höhere Teststärke als die Originalstudie aufweisen dürften, erscheint zweifelhaft, dass von zustandsbezogenem Optimismus und Pessimismus tatsächlich eine moderierende Wirkung auf den Sunk-Cost-Effekt ausgehen könnte.

In beiden Replikationsstudien ließ sich lediglich der generelle Haupteffekt der Originalstudie replizieren, wonach Optimisten eine höhere Investitionsneigung aufwiesen als Pessimisten. Dieser Befund kann wie schon erwähnt mehr oder weniger als erweiterte Konstruktvalidierung aufgefasst werden und tangiert nicht die eigentliche Fragestellung. Darüber hinaus ließ sich auch der von Juliusson (2006) berichtete Sunk-Cost-Haupteffekt auf der Stufe von Optimismus in beiden durchgeführten Studien nicht replizieren. Eine potenzielle Erklärung hierfür wäre, dass sich der Sunk-Cost-Effekt unter Umständen doch nicht so stabil nachweisen lässt wie allgemein angenommen (vgl. Friedman, Pommerenke, Lukose, Milam \& Huberman, 2007; Sleesman et al. 2012). 


\subsection{Regulatorischer Fokus: Promotion und Prevention}

Wie in Abschnitt 2.2.2.3 dargestellt, berichteten Molden und Hui (2011), dass ein Promotion Focus, im Sinne der regulatorischen Fokus Theorie von Higgins (1997), Eskalationstendenzen senken kann. Probanden, bei denen ein Promotion Focus auf StateEbene induziert wurde, investierten signifikant seltener in Arkes und Blumers (1985) Airplane-Szenario als Probanden mit einem Prevention Focus oder Probanden in der Kontrollgruppe. Die Autoren folgerten daraus, dass der Promotion Focus Personen dazu motiviere, weniger über anhaltende Verpflichtungen oder die Vermeidung von Verlusten nachzudenken, sondern vielmehr über erreichbare Möglichkeiten und Gewinne. Auf diese Weise könnten sie ihre Bindung zu Fehlern aus der Vergangenheit reduzieren, versunkene Kosten besser ignorieren und die Chance erhöhen, die besten Alternativen für die Zukunft auszuwählen.

Bei der Untersuchung von Molden und Hui (2011, Studie 2) ergibt sich allerdings die Problematik, dass die getroffene Schlussfolgerung hinsichtlich der versunkenen Kosten strenggenommen gar nicht durch das Studiendesign gestützt werden kann. Zwar wurde das gut etablierte Airplane-Szenario von Arkes und Blumer (1985) verwendet, jedoch nur in seiner $\mathrm{SC}^{(+)}$-Version, das heißt, es wurde keine vergleichende Faktorstufe ohne versunkene Kosten in das Design integriert. Wie in Abschnitt 2.1.3 dargelegt, handelt es sich hierbei um ein Vorgehen, das gelegentlich im Rahmen von Sunk-Cost-Untersuchungen vollzogen wird. Allerdings bleibt aufgrund der fehlenden Variation der versunkenen Kosten im Fall von Molden und Hui (2011) ungeklärt, ob die Probanden mit einem Promotion Focus versunkene Kosten tatsächlich besser ignorieren konnten, und damit robuster gegenüber dem Sunk-CostEffekt waren, als die übrigen Probanden. Stattdessen könnte der Promotion Focus auch unspezifisch die Investitionsfreudigkeit herabgesenkt haben. In diesem Fall würde es sich jedoch nicht um einen Moderator für den Sunk-Cost-Effekt handeln. 
Da bei Molden und Hui (2011) der regulatorische Fokus experimentell auf State-Ebene induziert wurde, wie bereits Optimismus vs. Pessimismus aus dem letzten Unterkapitel, wurden auch in diesem Fall zwei direkte Replikationsstudien durchgeführt, um genauere Aussagen über die Belastbarkeit der Originalbefunde auf State-Ebene treffen zu können (vgl. Kapitel 3). Beide Replikationsstudien wurden wie die Originalstudie als OnlineUntersuchungen realisiert. Um potenzielle Interaktionseffekte zwischen dem regulatorischen Fokus und den versunkenen Kosten aufdecken zu können, die wirklich auf eine moderierende Wirkung des regulatorischen Fokus auf den Sunk-Cost-Effekt hindeuten würden, wurde das originale Versuchsdesign von Molden und Hui (2011) um eine zusätzliche SC ${ }^{(-)}$-Faktorstufe erweitert. Die direkte Replikation ist dabei auf der Stufe vorhandener versunkener Kosten $\left(\mathrm{SC}^{(+)}\right)$vollständig gewährleistet.

\subsubsection{Erste Replikationsstudie}

\subsubsection{Methode}

\subsection{Stichprobe und Design}

Bei den Teilnehmern dieser Untersuchung handelte es sich überwiegend um Studierende verschiedener Studiengänge der Georg-August-Universität Göttingen. Wegen der Einführung einer ergänzenden $\mathrm{SC}^{(-)}$-Faktorstufe und für eine teststärkere Analyse, wurde der Stichprobenumfang im Vergleich zur Originalstudie vergrößert (287 Probanden gegenüber 114; davon 139 Probanden in den $\mathrm{SC}^{(+)}$-Replikationsbedingungen). Unter allen Teilnehmern befanden sich 216 weibliche (75\%) und 71 männliche (25\%) Versuchspersonen. Das Durchschnittsalter betrug 22,84 Jahre $(S D=4,35)$, in einer Spanne zwischen 16 und 58 Jahren. Wie in der Originalstudie wurde die Nationalität erhoben, demnach waren 95,5\% aller Teilnehmer Deutsche, 2,1\% stammten aus dem europäischen Ausland und 2,4\% aus Asien. 
Als höchsten Bildungsabschluss gaben 1,4\% die mittlere Reife, 76,3\% das Abitur und 22,3\% einen Bachelorabschluss oder höher an. ${ }^{25}$

Wie erwähnt wurde das originale Versuchsdesign von Molden und Hui (2011, Studie 2) übernommen und um eine $\mathrm{SC}^{(-)}$-Faktorstufe ergänzt. Im Vergleich zur Originalstudie ergab sich hierdurch ein differenzierteres 3 (regulatorischer Fokus: Promotion vs. Prevention vs. Neutral $) \times 2$ (versunkene Kosten: vorhanden vs. nicht vorhanden) - Zwischensubjektdesign. Die dichotome Investitionsentscheidung (ja vs. nein) wurde als Kriteriumsvariable mithilfe des vollständigen Airplane-Szenarios von Arkes und Blumer (1985) gemessen.

\subsection{Durchführung und Material}

Die Probandenakquise erfolgte persönlich in verschiedenen Vorlesungen, am schwarzen Brett der Universität sowie über Onlineplattformen. Den potenziellen Teilnehmern wurde dabei mitgeteilt, dass es sich um einen Fragebogen zum „Entscheidungsverhalten“ handele. Außerdem erhielten sie Hinweise zur Anonymität und der Versuchspersonenvergütung. Für ihre Teilnahme erhielten die Probanden entweder eine halbe Versuchspersonenstunde im Rahmen des Bachelor-Psychologiestudiums oder nahmen an einer Verlosung von zehn mal 20 Euro teil.

Für die onlinebasierte Darstellung des Fragebogens wurde wieder die Open-SourcePlattform „LimeSurvey“ genutzt (http://www.limesurvey.org). Da zum Erhebungszeitpunkt keine Möglichkeit bestand, hierin verschiedene Versuchsbedingungen unter derselben Fragebogenadresse $\mathrm{zu}$ definieren, wurde das $3 \times 2$ Design in Form von sechs

\footnotetext{
${ }^{25}$ Die SC ${ }^{(+)}$-Replikationsbedingungen enthielten 139 Datensätze mit 109 weiblichen (78\%) und 30 männlichen (22\%) Probanden. Das durchschnittliche Alter betrug darin 22,13 Jahre $(S D=2,90)$, in einer Spanne zwischen 16 und 36 Jahren. 95\% dieser Teilnehmer waren deutsch, 2,9\% stammten aus dem europäischen Ausland und 2,2\% aus Asien. Als höchsten Bildungsabschluss gaben 0,7\% die mittlere Reife, 77\% Abitur und 22,3\% einen Bachelorabschluss oder höher an. In den Analysen konnten nur vollständig ausgefüllte Fragebögen mit einbezogen werden, was im Rahmen der Onlineerhebung durch selbständige Abbrüche der Teilnehmer nicht in allen Fällen gegeben war. Neun Datensätze mussten unabhängig davon aus den Analysen ausgeschlossen werden: In zwei Fällen ließen Probanden das Feld im Rahmen der Schreibaufgabe unausgefüllt, die der Manipulation des regulatorischen Fokus dienen sollte. Eine Person hat das Experiment zweimal bearbeitet und kannte so bereits die Inhalte bei der zweiten Bearbeitung, weshalb nur die Daten der Erstteilnahme berücksichtig wurden. Sechs weitere Versuchspersonen beschrieben im Rahmen des Suspicion Checks relativ konkret, dass es sich um ein Sunk-Cost-Experiment handeln könnte und wurden daher ebenfalls ausgeschlossen.
} 
Einzelfragebögen realisiert. Beim Öffnen des kommunizierten Hauptlinks wurden die Probanden mithilfe eines zwischengeschalteten Skripts zufällig zu einem dieser sechs Fragebögen weitergeleitet.

Die ersten drei Seiten des Fragebogens waren in allen Versuchsbedingungen identisch: Auf der ersten Fragebogenseite informierten schriftliche Instruktionen über die Struktur des Fragebogens, die Versuchspersonenvergütung und die Anonymität. Durch einen Kick auf „Weiter“ gelangten die Teilnehmer zur zweiten Fragebogenseite. Hierauf wurden sie gebeten, einige Pflichtangaben zu ihrer Personen zu machen. Abgefragt wurden das Geschlecht, das Alter, die Nationalität, die Muttersprache und der höchste Bildungsabschluss mit den vorgegebenen Antwortmöglichkeiten: „Realschulabschluss“, „Abitur“, „Bachelor-Abschluss“, „Master-Abschluss“, „Doktortitel“ oder „Sonstiges“ (verbunden mit einem offenen Textfeld). Eine weitere offene Frage, nach dem aktuellen bzw. abgeschlossenen Studium, war nur von Studierenden bzw. Absolventen zu beantworten. Auf der dritten Fragebogenseite folgte eine kurze Erklärung der folgenden fünfminütigen Aufgabe. Die Probanden wurden gebeten, sicherzustellen, diese ohne Unterbrechung bearbeiten zu können. Mit einem Klick auf „Ich bin bereit" wurde die Aufgabenbearbeitung gestartet.

Es öffnete sich die vierte Fragebogenseite mit einem offenen Textfeld, in das die Probanden einen kurzen Aufsatz schreiben sollten. Inhaltlich handelte es sich um die gleiche Schreibaufgabe zur experimentellen Manipulation des regulatorischen Fokus, wie sie von Molden und Hui (2011) verwendet wurde. Probanden der Promotion-Bedingungen wurden gebeten, über ihre „Ziele und Hoffnungen“ zu berichten, Probanden der PreventionBedingungen schrieben hingegen über ihre „Aufgaben und Verpflichtungen“ und Probanden in den Kontrollbedingungen über ihre „typischen täglichen Aktivitäten“.

Neben der Information, dass man nach Ablauf der zur Verfügung stehenden fünf Minuten automatisch zur nächsten Seite navigiert würde, zeigte ein Counter parallel die noch 
verbleibende Schreibzeit an. Nach Ablauf von vier Minuten erschien ein zusätzliches Informationsfenster oberhalb des Textfeldes, das auf die letzte verbleibende Minute hinwies.

Nach Beendigung der fünfminütigen Schreibaufgabe wurden die Probanden auf die fünfte Fragebogenseite weitergeleitet. Hierauf bearbeiteten sie Arkes und Blumers (1985) AirplaneSzenario, je nach Versuchsbedingung entweder in der $\mathrm{SC}^{(+)}$- oder $\mathrm{SC}^{(-)}$-Version. Die Frage, ob eine Million Euro in das Projekt investiert werden solle, beantworteten die Probanden durch das Anklicken einer der beiden Antwortmöglichkeiten ,ja“" oder „nein“.

Nach der Bearbeitung des Sunk-Cost-Szenarios waren alle Daten wie in der Originalstudie erhoben. Darüber hinausgehend folgte im Anschluss noch eine sechste Fragebogenseite, die wiederum für alle Probanden identisch war. Hierauf sollten sie angeben, ob die Fragen verständlich waren, ob die Befragung reibungslos abgelaufen ist und ob ihnen beim Ausfüllen etwas aufgefallen sei. Diese Abfragen dienten einer besseren Einschätzung über die Verwertbarkeit der Daten, welche auf Basis der Antworten aber in allen Fällen gegeben war. Außerdem wurden die Probanden im Sinne einer Verdachtskontrolle gebeten, in einem offenen Textfeld niederzuschreiben, worum es ihrer Meinung nach inhaltlich in der Studie ging. Anschließend folgte eine Auflistung zur Abfrage der gewünschten Probandenvergütung. Hier musste einer der folgenden drei Punkte ausgewählt werden: „Halbe Versuchspersonenstunde“, „Verlosung“" oder „Keines von beidem“.

Den Abschluss der Befragung bildete die optionale Angabe einer E-Mail-Adresse. Die Adressenerhebung diente der Kontaktaufnahme über verschiedene Verteiler per Blindkopie: Zum einen wurden die Gewinner aus der Verlosung von zehn mal 20 Euro kontaktiert sowie die Psychologiestudierenden, die alternativ eine halbe Versuchspersonenstunde als Vergütung wünschten. Zum anderen wurden alle Probanden nach Abschluss der Erhebungen beider Replikationsstudien per E-Mail über die Hintergründe der Untersuchung aufgeklärt. Durch einen Klick auf „Absenden“ gelangten die Probanden zur letzten Seite. Hierauf wurde ihnen 
für die Teilnahme gedankt und eine Kontaktadresse der Versuchsleitung für eventuelle Rückfragen kommuniziert.

\subsubsection{Ergebnisse}

Die dichotome Investitionsentscheidung wurde als Kriteriumsvariable mit „0“ (nicht investieren) und „1“ (investieren) codiert. Die Codierung der induzierten Motivation als erstem Faktor lautete „1“ (Promotion), „2“ (Prevention) und „,““ (Kontrollgruppe), die der versunkenen Kosten als zweitem Faktor „0“ $\left(\mathrm{SC}^{(-)}\right)$und „1“ $\left(\mathrm{SC}^{(+)}\right)$.

Die Berichte der Analysen erfolgen in zwei Schritten. Um eine bestmögliche Vergleichbarkeit zu den Befunden von Molden und Hui (2011) zu gewährleisten, wird zunächst eine Analyse der reinen $\mathrm{SC}^{(+)}$-Replikationsbedingungen berichtet, was dem Originaldesign entspricht. Im Anschluss folgt eine Analyse des erweiterten Designs der vorliegenden Replikationsstudie, welches beide Faktorstufen der versunkenen Kosten umfasste, um potenzielle Interaktionseffekte nachweisen zu können.

\subsection{Analyse der reinen Replikationsbedingungen}

Wie in der Originalstudie wurden Kontingenztabellen-Analysen auf Stufe vorhandener versunkener Kosten $\left(\mathrm{SC}^{(+)}\right)$durchgeführt, um die Originalbefunde $\mathrm{zu}$ replizieren. Die berichteten Effekte von Molden und Hui (2011) sind in Tabelle 8 zusammengefasst, um einen direkten Vergleich zu ermöglichen.

Effects of contingency table analyses

\begin{tabular}{ll}
\hline Motivation & $\chi^{2}(2, N=114)=6.76, p=.03$ \\
\hline Difference Promotion vs. Prevention & $\chi^{2}(1, N=78)=3.85, p=.05$ \\
\hline Difference Promotion vs. Control Group & $\chi^{2}(1, N=75)=5.36, p=.02$ \\
\hline Difference Prevention vs. Control Group & $\chi^{2}(1, N=75)=0.18, p=.67$ \\
\hline Probability of Investment (Promotion) & $\chi^{2}(1, N=39)=1.25, p=.26$ \\
\hline Probability of Investment (Prevention) & $\chi^{2}(1, N=35)=13.56, p<.001$ \\
\hline Probability of Investment (Control Group) & $\chi^{2}(1, N=39)=16.00, p<.001$ \\
\hline
\end{tabular}

Tabelle 8: Befunde aus den Analysen von Molden und Hui (2011) mit der Investitionsentscheidung als abhängige Variable. 
In der Originalstudie wurde ein Haupteffekt für die induzierte Motivation (regulatorischer Fokus) berichtet, der darin bestand, dass die Probanden mit einem Promotion Focus jeweils signifikant seltener investierten als die Probanden mit einem Prevention Focus oder Probanden in der Kontrollgruppe. Hierfür fanden sich in der Replikationsstudie allerdings keine Anzeichen. Es konnte auf der $\mathrm{SC}^{(+)}$-Faktorstufe kein Haupteffekt für den regulatorischen Fokus nachgewiesen werden $\left(\chi^{2}[2, N=139]=1.82, p=.402\right)$. Rein deskriptiv war sogar das Gegenteil der Fall, da die Probanden mit einem Promotion Focus hier häufiger investierten als die Probanden mit einem Prevention Focus oder die Probanden der Kontrollgruppe. In paarweisen Vergleichen zwischen den drei Faktorstufen zeigten sich diese Unterschiede, im Gegensatz zur Originalstudie, nicht signifikant (Promotion vs. Prevention: $\quad \chi^{2}[1, N=90]=1.81, p=.178 ;$ Promotion vs. Control: $\chi^{2}[1, N=94]=.83$, $p=.362$ ). Einen Unterschied zwischen den Probanden mit einem Prevention Focus und den Probanden in der Kontrollgruppe gab es weder in der Originalstudie noch in der Replikationsstudie $\left(\chi^{2}[1, N=94]=.22, p=.639\right)$.

Abbildung 3 stellt die Investitionsunterschiede zwischen den Faktorstufen anhand der mittleren prozentualen Investitionsrate für beide Studien grafisch gegenüber.
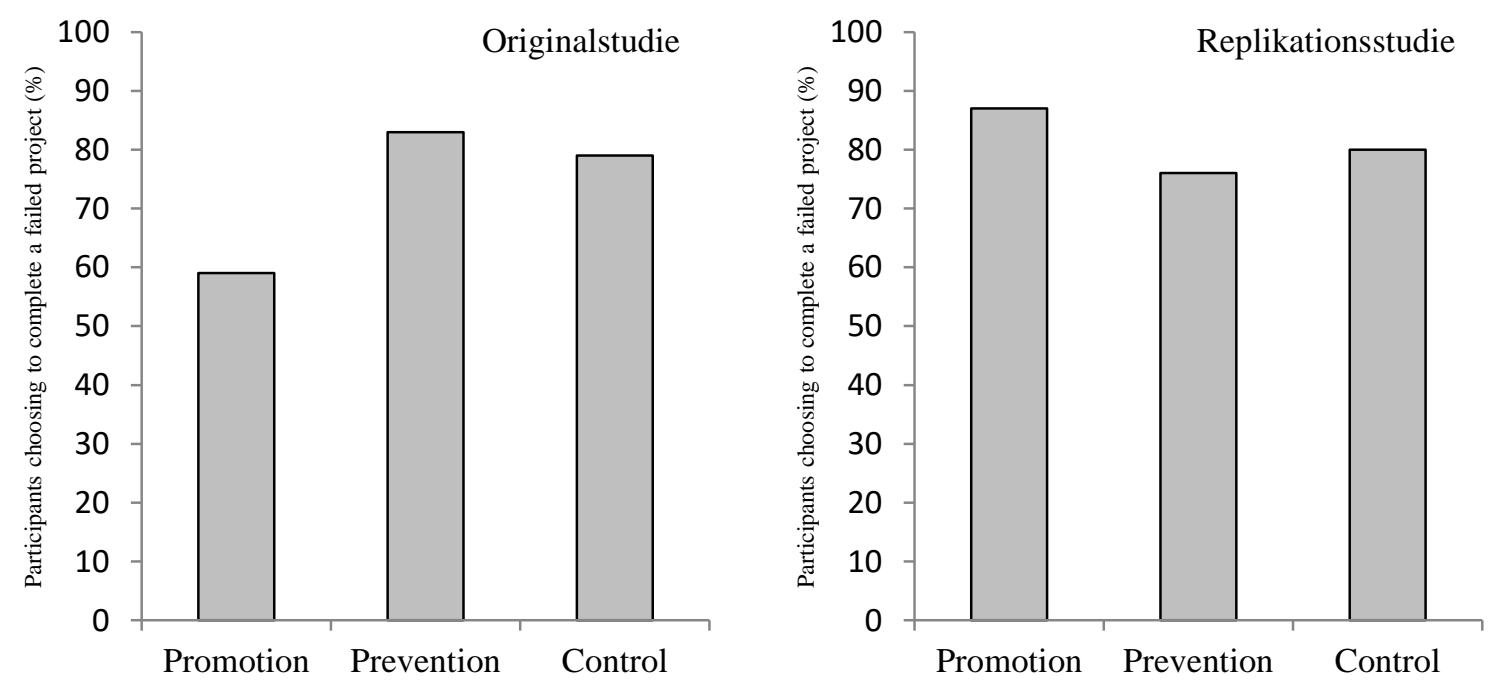

Abbildung 3: Vergleich der Ergebnisse aus Moldens und Huis (2011) Originalstudie mit denen aus der ersten Replikationsstudie. Auf der Ordinate ist jeweils die durchschnittliche Investitionsrate in Prozent abgetragen, die Abszisse ist nach den drei Motivationsstufen unterteilt. 
Im Originalartikel wurde außerdem angemerkt, dass es nur bei einem Promotion Focus kein signifikantes Überwiegen von Entscheidungen für oder gegen eine Investition gab. Bei einem Prevention Focus und in der Kontrollgruppe tätigten die Probanden die Investition hingegen signifikant häufiger als das Projekt abzubrechen. In der vorliegenden Replikationsstudie fand sich jedoch auf allen drei Faktorstufen ein signifikantes Überwiegen von Entscheidungen für eine Investition (vgl. Abbildung 3; Prevention: $\chi^{2}[1, N=45]=11.76$, $p=.001$, Control: $\left.\chi^{2}[1, N=49]=17.16, p<.001\right)$, wobei der Unterschied für den Promotion Focus sogar am deutlichsten ausfiel $\left(\chi^{2}[1, N=45]=24.20, p<.001\right){ }^{26}$

\subsection{Analyse des erweiterten Versuchsdesigns}

Um den Sunk-Cost-Effekt sowie eine mögliche Interaktion zwischen den versunkenen Kosten und dem regulatorischen Fokus nachweisen zu können, wurde das in der Replikationsstudie eingeführte erweiterte Versuchsdesign (inklusive der $\mathrm{SC}^{(-)}$-Faktorstufe) mithilfe einer logistischen Regression analysiert.

Dabei zeigte sich ein Sunk-Cost-Haupteffekt $\left(B=3.69, S E=.38 ;\right.$ Wald $\chi^{2}[1, N=287]=$ 93.76, $p<.001$, OR $=40.08)$, wonach auf der Stufe mit versunkenen Kosten signifikant häufiger investiert wurde als auf der Stufe ohne versunkene Kosten $\left(M_{(S C+)}=80,6 \%\right.$ vs. $M_{(S C-)}$ = 11,5\%; vgl. Abbildung 4). Der grundlegende Befund von Arkes und Blumer (1985) konnte daher repliziert werden. Eine Interaktion zwischen den versunkenen Kosten und dem regulatorischen Fokus blieb hingegen aus $\left(\right.$ Wald $\left.\chi^{2}[2, N=287]=1.85, p=.397\right)$. An dieser Stelle deutet also nichts darauf hin, dass von dem regulatorischen Fokus ein moderierender Einfluss auf den Sunk-Cost-Effekt ausgehen könnte.

In dieser erweiterten Analyse war nun auch ein Haupteffekt für die Motivation nachweisbar $\left(\right.$ Wald $\left.\chi^{2}[2, N=287]=6.95, p=.031\right)$. Probanden mit einem Promotion Focus

\footnotetext{
${ }^{26}$ Wie in der Originalstudie hatten die Variablen Geschlecht, Alter, Nationalität und Bildungsniveau keinen Einfluss auf die zentrale Befundlage dieser Analysen, ebenso wenig wie der Studiengang als ergänzende Kontrollvariable.
} 
investierten im Durchschnitt sowohl signifikant häufiger als Probanden mit einem Prevention

Focus $\left(M_{(\text {Prevention })}=40,2 \%\right.$ vs. $M_{(\text {Promotion })}=52,7 \% ; B=-.83, S E=.41 ;$ Wald $\chi^{2}[1, N=190]=$ 4.12, $p=.042$, OR = .44) als auch signifikant häufiger als die Probanden in der Kontrollgruppe $\left(M_{(\text {Control })}=42,3 \%\right.$ vs. $M_{(\text {Promotion })}=52,7 \% ; B=-1.16, S E=.49 ;$ Wald $\chi^{2}[1$, $N=190]=5.53, p=.019$, OR $=.32$ ). Diese Ergebnisse stehen allerdings im direkten Gegensatz zur Originalstudie, in der die Probanden mit einem Promotion Focus eigentlich eine signifikant niedrigere Investitionsneigung aufwiesen als die Probanden der Vergleichsbedingungen.

Für einen besseren Überblick veranschaulicht Abbildung 4 die mittleren prozentualen Investitionsraten aller sechs Versuchsbedingungen aus der Replikationsstudie grafisch. ${ }^{27}$

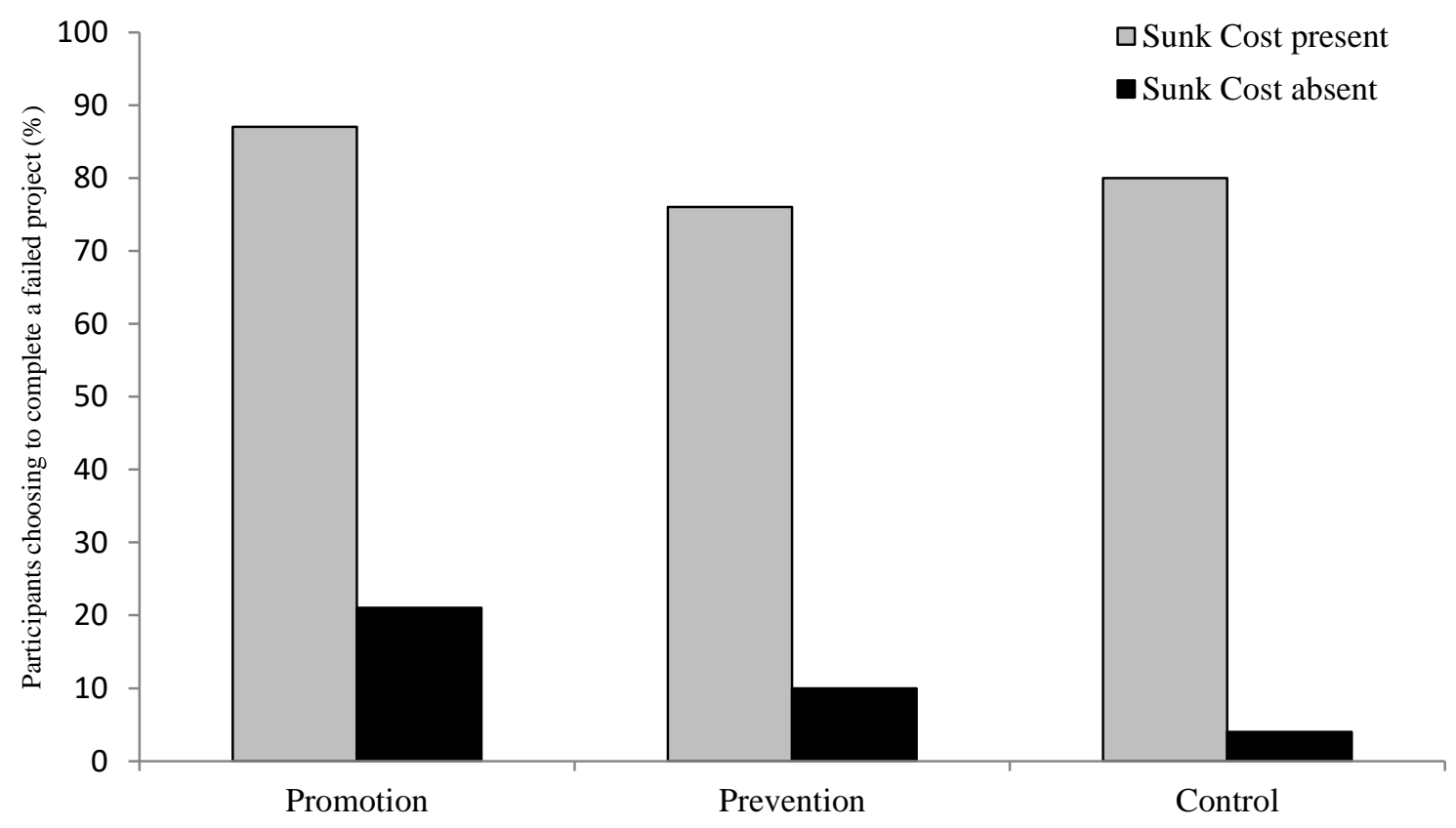

Abbildung 4: Vergleich der durchschnittlichen prozentualen Investitionsraten aller Versuchsbedingungen aus der ersten Replikationsstudie zu Molden und Hui (2011), unterteilt nach den drei Stufen des regulatorischen Fokus sowie beiden Stufen der versunkenen Kosten $\left(\right.$ grau $=S C^{(+)}$, schwarz $\left.=S C^{(-)}\right)$.

\footnotetext{
${ }^{27}$ Hinsichtlich des Überwiegens von Entscheidungen für oder gegen eine Investition, fand sich bei Betrachtung beider SC-Faktorstufen erwartungsgemäß kein Unterschied mehr auf den Motivationsstufen. Unter Einschluss der Kontrollvariablen Geschlecht, Alter, Nationalität, Bildungsniveau und Studiengang zeigte sich der Investitionsunterschied zwischen den Stufen Promotion und Prevention nur noch marginal $(B=-.77, S E=.41$; Wald $\chi^{2}[1, N=190]=3.58, p=.059$, OR = .46). Das gleiche Ergebnis fand sich bei einer Hinzunahme jener sechs Probanden, die in der Verdachtskontrolle den Sunk-Cost-Effekt vermuteten und daher von den Hauptanalysen ausgeschlossen wurden $\left(B=-.72, S E=.39 ;\right.$ Wald $\chi^{2}[1, N=193]=3.43, p=.064$, OR $\left.=.49\right)$. Dieses war allerdings die einzige Abweichung aus den Kontrollanalysen zur zentralen Befundlage. Unabhängig davon bleibt festzuhalten, dass die Originalbefunde nicht repliziert werden konnten.
} 


\subsubsection{Diskussion}

Molden und Hui (2011) berichteten, dass ein induzierter Promotion Focus hilfreich sei, um versunkene Kosten besser zu ignorieren. Diese Aussage kann jedoch strenggenommen schon durch die Originalstudie nicht gestützt werden, da hierin die versunkenen Kosten innerhalb des Airplane-Szenarios nicht variiert wurden. Um die Frage nach der Wirkung des regulatorischen Fokus auf den Sunk-Cost-Effekt besser beantworten zu können, kam in der vorliegenden Replikationsstudie daher ein verbessertes Design zum Einsatz, welches das $\mathrm{SC}^{(+)}$-Originaldesign um eine $\mathrm{SC}^{(-)}$-Faktorstufe erweiterte.

Innerhalb dieses erweiterten Designs ließ sich ein signifikanter Sunk-Cost-Haupteffekt nachweisen, welcher Arkes und Blumers (1985) grundlegendem Befund für das AirplaneSzenario entsprach. Es zeigte sich jedoch keine Interaktion zwischen den versunkenen Kosten und dem regulatorischen Fokus aus der hervorginge, dass ein Promotion Focus die SunkCost-Neigung reduziert.

Auch der grundlegende Befund von Molden und Hui (2011), wonach Probanden mit einem Promotion Focus eine signifikant niedrigere Investitionsneigung aufwiesen als Probanden mit einem Prevention Focus oder Probanden der Kontrollgruppe, konnte nicht repliziert werden. In der Replikationsstudie, die aufgrund der größeren Stichprobe eine tendenziell höhere Teststärke besaß als die Originalstudie, trat genau das Gegenteil ein: Hierin wiesen die Probanden mit einem Promotion Focus sogar eine höhere Investitionsneigung als die Probanden mit einem Prevention Focus oder die Probanden der Kontrollgruppe auf. Diese Unterschiede zeigten sich allerdings erst in der Analyse des erweiterten Designs und nicht in der Analyse der reinen $\mathrm{SC}^{(+)}$-Replikationsbedingungen.

In Anbetracht der gegenteiligen Ergebnisse beider Studien, kann an dieser Stelle keine verlässliche Aussage darüber getroffen werden, ob ein Promotion Focus die Investitionsneigung entweder erhöht oder absenkt. Hinsichtlich der zentralen Fragestellung lässt sich jedoch festhalten, dass sich in der Replikationsstudie keine Anhaltspunkte dafür 
fanden, dass es sich bei dem regulatorischen Fokus um eine Moderatorvariable für den Sunk-

Cost-Effekt handeln könnte. Weiteren Aufschluss hierüber kann die zweite geplante Replikationsstudie geben, über die im nun folgenden Abschnitt berichtet wird.

\subsubsection{Zweite Replikationsstudie}

In dieser zweiten Online-Replikationsstudie wurden alle Aspekte der ersten wiederholt. Anstelle des Airplane-Szenarios kam hier jedoch das Druckerpressen-Szenario von Arkes und Blumer (1985) zum Einsatz, um zu überprüfen, ob die Originalbefunde in einem anderen, unabhängigen Sunk-Cost-Szenario repliziert werden können.

\subsubsection{Methode}

\subsection{Stichprobe und Design}

Auch bei den Teilnehmern dieser Untersuchung handelte es sich überwiegend um Studierende verschiedener Fachrichtungen der Georg-August-Universität Göttingen. Insgesamt nahmen hieran 242 Personen teil, davon 121 in den $\mathrm{SC}^{(+)}$Replikationsbedingungen. 181 Probanden waren weiblich (75\%) und 61 männlich (25\%). Das Durchschnittsalter betrug 22,89 Jahre $(S D=2,83$ ), in einer Spanne zwischen 16 und 33 Jahren. 97,1\% der Teilnehmer waren Deutsche, 1,7\% stammten aus dem europäischen Ausland, 0,4\% aus Asien und 0,8\% aus Südamerika. Das Bildungsniveau reichte von der Fachhochschulreife bis zum Doktorgrad (71,5\% gaben die Fachhochschulreife oder Abitur als höchsten Bildungsabschluss an, 28,5\% besaßen bereits einen Bachelorabschluss oder höher). ${ }^{28}$

\footnotetext{
${ }^{28}$ Die SC ${ }^{(+)}$-Replikationsbedingungen enthielten 121 Datensätze mit 89 weiblichen $(74 \%)$ und 32 männlichen (26\%) Probanden. Das Durchschnittsalter betrug in dieser Untergruppe 22,83 Jahre $(S D=2,77)$, in einer Spanne zwischen 17 und 32 Jahren. Der Anteil deutscher Teilnehmer betrug 95,9\%, aus dem europäischen Ausland stammten 2,5\% und jeweils 0,8\% aus Asien und Südamerika. 74.4\% besaßen die Fachhochschulreife oder Abitur und 25,6\% einen Bachelorabschluss oder höher. Auch in dieser Studie konnten nur vollständig ausgefüllte Fragebögen in den Analysen berücksichtigt werden. Neun Datensätze mussten wegen Mehrfachteilnahmen ausgeschlossen werden, wobei jeweils nur die zuerst bearbeiteten Fragebögen berücksichtigt wurden. Eine weitere Versuchsperson wurde ausgeschlossen, da sie keinen Text in der Schreibaufgabe eingegeben hatte, die eigentlich der Manipulation des regulativen Fokus dienen sollte.
} 
Das erweiterte $3 \times 2$ Versuchsdesign war identisch $\mathrm{zu}$ jenem aus der ersten Replikationsstudie. Allerdings wurde die dichotome Investitionsentscheidung (ja vs. nein) als Kriteriumsvariable diesmal mit Arkes und Blumers (1985) Druckerpressen-Szenario erhoben.

\subsection{Durchführung und Material}

Sowohl die Akquise als auch die Entlohnung entsprachen denen aus der ersten Replikationsstudie. Auch die Durchführung gelang wieder mit sechs Einzelfragebögen über die Online-Plattform „LimeSurvey“ (http://www.limesurvey.org), auf die die Probanden durch ein zwischengeschaltetes Skript zufällig weitergeleitet wurden.

Der Inhalt der Fragebögen entsprach ebenfalls dem aus der ersten Replikationsstudie mit folgenden Unterschieden: Erstens wurde, wie erwähnt, das Airplane-Szenario gegen das Druckerpressen-Szenario von Arkes und Blumer (1985) getauscht. Zweitens wurde direkt im Anschluss an die experimentelle Manipulation des regulativen Fokus auch seine chronische Ausprägung auf Trait-Ebene mithilfe einer von Lockwood, Jordan und Kunda (2002) entwickelte Skala gemessen. Die in dieser Studie verwendete deutsche Version der Skala stammt von Keller und Bless (2006) und umfasst je neun Items zu Promotion und Prevention auf Trait-Ebene. ${ }^{29}$ Die dritte Veränderung betraf den Abschlussfragebogen, in dem die Frage nach etwaigen Auffälligkeiten gestrichen wurde. Neu hinzu kam stattdessen die Abfrage, ob schon einmal an einer ähnlichen Studie teilgenommen wurde und falls ja, worum es darin ging. Aufgrund der Fragen im Abschlussfragebogen musste allerdings kein Proband aus den Analysen ausgeschlossen werden.

\footnotetext{
29 Über die Originalstudie hinausgehend, sollte auf diesem Wege eine Manipulationskontrolle durchgeführt werden. Dieses erfolgte explorativ und in Kenntnis darüber, dass ein Trait-Fragebogen kein sensitives Maß für eine State-Untersuchung darstellen muss. Bei dieser Untersuchung konnten jedoch keine signifikanten Zusammenhänge zwischen den jeweiligen Trait- und State-Maßen festgestellt werden, weder für Promotion noch für Prevention. Auf eine Diskussion der Befunde wird verzichtet, da in der Originalstudie selbst keine Anstrengungen hinsichtlich einer Manipulationskontrolle berichtet wurden. Es ist daher unklar, ob die Manipulation bei Molden und Hui (2011) überhaupt erfolgreich war, bzw. anhand des hier angewendeten Verfahrens bestätigt worden wäre. Eine detailliertere Beschreibung dieser Analyse findet sich im Anhang B.
} 


\subsubsection{Ergebnisse}

Die durchgeführten Analysen sowie die Codierung der Variablen entsprechen denen der ersten Replikationsstudie (vgl. Abschnitt 4.3.1.2), daher werden auch in diesem Abschnitt zunächst die Analysen der reinen $\mathrm{SC}^{(+)}$-Replikationsbedingungen berichtet und im Anschluss die Analyse des erweiterten Designs, welches um eine $\mathrm{SC}^{(-)}$-Faktorstufe ergänzt wurde.

\subsection{Analyse der reinen Replikationsbedingungen}

Der zentrale Haupteffekt für die Motivation aus Moldens und Huis (2011) Originalstudie, wonach die Probanden mit einem Promotion Focus signifikant seltener investierten als die Probanden in den jeweiligen Vergleichsbedingungen, war auch in dieser Untersuchung nicht replizierbar $\quad\left(\chi^{2}[2, N=121]=.40, p=.819\right)$. In paarweisen Vergleichen $\quad$ der drei Motivationsstufen untereinander zeigten sich keine Unterschiede hinsichtlich der Investitionsneigung (Promotion vs. Control: $\chi^{2}[1, N=81]=.30, p=.585$; Prevention vs. Control: $\left.\chi^{2}[1, N=81]=.30, p=.585\right)$, die Probanden mit einem Promotion Focus und die Probanden mit einem Prevention Focus verhielten sich sogar absolut identisch $\left(\chi^{2}[1, N=80]<.001, p=1.000\right)$. Gleichzeitig fand sich in keiner der drei Motivationsbedingungen ein signifikantes Überwiegen von Entscheidungen für oder gegen eine Investition (Promotion: $\left(\chi^{2}[1, N=40]=.10, p=.752\right.$; Prevention: $\chi^{2}[1, N=40]=.10$, $p=.752 ;$ Control: $\left.\chi^{2}[1, N=41]=1.20, p=.274\right)$.

\subsection{Analyse des erweiterten Versuchsdesigns}

In der Analyse des erweiterten Designs zeigte sich ein signifikanter Sunk-CostHaupteffekt $\left(B=-2.11, S E=.38 ;\right.$ Wald $\chi^{2}[1, N=242]=31.11, p<.001$, OR $\left.=.12\right)$ in der Form, dass die Probanden auf Stufe vorhandener versunkener Kosten seltener investierten als auf der Stufe ohne versunkene Kosten $\left(M_{(S C+)}=58,5 \%\right.$ vs. $M_{\left(S C_{-}\right)}=90,1 \%$; vgl. Abbildung 5). Das bedeutet, dass Arkes und Blumers (1985) grundlegender Befund für das DruckerpressenSzenario repliziert werden konnte. Allerdings fanden sich auch im Rahmen dieser zweiten Replikationsstudie keine Indizien für einen moderierenden Einfluss des regulatorischen Fokus 
auf den Sunk-Cost-Effekt, da eine entsprechende Interaktion ausblieb (Wald $\chi^{2}[2, N=242]=$ $1.62, p=.445)$.

Darüber hinaus konnte auch in dieser erweiterten Analyse der Haupteffekt für die Motivation aus der Originalstudie nicht repliziert werden (Wald $\chi^{2}[2, N=242]=$ 1.05, $p=.591)$. Die durchschnittlichen Investitionsraten waren in allen drei Motivationsbedingungen ähnlich hoch, weshalb auch die von Molden und Hui (2011) berichteten Investitionsunterschiede zwischen den Probanden mit einem Promotion Focus einerseits und den Probanden mit einem Prevention Focus oder denen in der Kontrollgruppe andererseits nicht repliziert werden konnten $\left(M_{(\text {Prevention })}=73,8 \%\right.$ vs. $M_{(\text {Promotion })}=70,0 \%$; $B=.50, S E=.49 ;$ Wald $\chi^{2}[1, N=160]=1.04, p=.307$, OR $=1.65 ; M_{(\text {Control })}=73,2 \%$ vs. $M_{(\text {Promotion })}=70,0 \% ; B=.14, S E=.41 ;$ Wald $\left.\chi^{2}[1, N=162]=.11, p=.736, \mathrm{OR}=1.15\right)$.

Für einen besseren Überblick veranschaulicht Abbildung 5 die mittleren prozentualen Investitionsraten aller sechs Versuchsbedingungen dieser zweiten Replikationsstudie grafisch. ${ }^{30}$

${ }^{30}$ Die Variablen Geschlecht, Alter, Nationalität und Bildungsniveau hatten wie in der Originalstudie keinen Einfluss auf die zentrale Befundlage der Analysen beider Designs, ebenso wenig wie der Studiengang als ergänzende Kontrollvariable. 


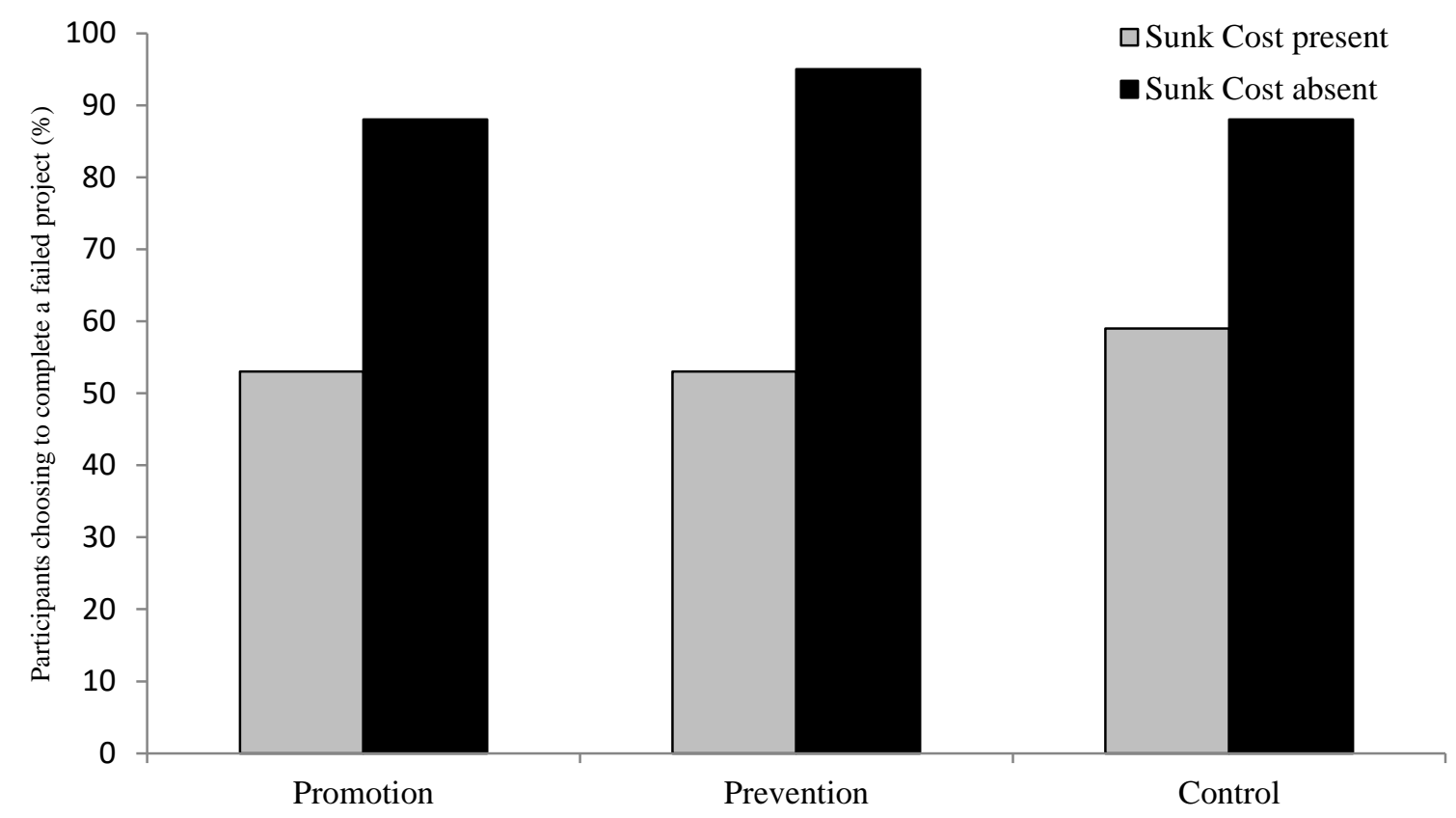

Abbildung 5: Vergleich der durchschnittlichen prozentualen Investitionsraten aller Versuchsbedingungen aus der zweiten Replikationsstudie zu Molden und Hui (2011), unterteilt nach den drei Stufen des regulatorischen Fokus sowie beiden Stufen der versunkenen Kosten $\left(\right.$ grau $=S C^{(+)}$, schwarz $\left.=S C^{(-)}\right)$.

\subsubsection{Diskussion}

Auch die Ergebnisse dieser zweiten Replikationsstudie können die Aussage von Molden und Hui (2011) nicht stützen, dass ein Promotion Focus hilfreich sei, um versunkene Kosten besser zu ignorieren. Mithilfe des um eine $\mathrm{SC}^{(-)}$-Faktorstufe erweiterten Versuchsdesigns konnte zwar der grundlegende Sunk-Cost-Effekt für das Druckerpressen-Szenario von Arkes und Blumer (1985) repliziert werden, jedoch blieb die zentrale Interaktion zwischen den versunkenen Kosten und dem regulatorischen Fokus aus, wie schon in der ersten Replikationsstudie. Auf Basis der Ergebnisse beider Replikationsstudien fanden sich also keinerlei Indizien, dass der zustandsbezogene regulative Fokus tatsächlich eine moderierende Wirkung auf den Sunk-Cost-Effekt haben könnte.

Ferner konnten auch die generellen Investitionseffekte aus der Originalstudie von Molden und Hui (2011) in beiden Replikationsstudien nicht repliziert werden, bei denen die Probanden mit einem Promotion Focus eine geringere Investitionsneigung aufwiesen als Probanden mit einem Prevention Focus oder Probanden in der Kontrollgruppe. In der ersten Replikationsstudie fanden sich, trotz Verwendung des gleichen Szenarios wie in der 
Originalstudie, genau gegenteilige Ergebnisse. In der zweiten Replikationsstudie blieben signifikante Unterschiede innerhalb des Druckerpressen-Szenarios ganz aus. In dem Zusammenhang sei noch einmal betont, dass beide Replikationsstudien durch ihre größeren Stichprobenumfänge näherungsweise eine höhere Teststärke aufweisen als die Originalstudie und die vorliegenden Ergebnisse daher durchaus verlässlich erscheinen. 


\subsection{Rational Thinking Style}

In Abschnitt 2.2.2.4 wurde die Untersuchung von Wong et al. (2008, Studie 1) vorgestellt. Die Autoren berichteten eine stärkere Investitionsbereitschaft für Personen mit hohen TraitAusprägungen des Rational Thinking Styles (vgl. Pacini und Epsteins, 1999) als für Personen mit niedrigen Ausprägungen. Dieser Befund war vor allem auf die Fähigkeitskomponente zum rationalen Denken (Rational Ability) zurückzuführen und nicht auf die motivationale Komponente (Rational Engagement). Für ihre Erhebung verwendeten die Autoren eine modifizierte Version des Airplane-Szenarios von Arkes und Blumer (1985).

Im Rahmen der Studie wurde allerdings, wie bereits in der eben diskutierten Studie zum regulatorischen Fokus, nur eine $\mathrm{SC}^{(+)}$-Version des Szenarios eingesetzt (vgl. Abschnitt 2.1.3). Auf Basis des Originaldesigns ist daher auch in diesem Fall nicht feststellbar, ob der Rational Thinking Style bei Wong et al. (2008) pauschal die Investitionsfreudigkeit erhöhte oder als spezifischer Moderator für den Sunk-Cost-Effekt angesehen werden kann. Daher wurde im Zuge der Replikationsstudie, die in diesem Unterkapitel vorgestellt wird, eine kontrastierende $\mathrm{SC}^{(-)}$-Faktorstufe in das Versuchsdesign mit aufgenommen, um potenzielle Interaktionseffekte zwischen dem Rational Thinking Style und den versunkenen Kosten aufdecken zu können. Die direkte Replikation ist dabei wieder auf der Stufe vorhandener versunkener Kosten $\left(\mathrm{SC}^{(+)}\right)$vollständig gewährleistet.

\subsubsection{Methode}

\subsubsection{Stichprobe und Design}

Bei den Teilnehmern dieser Replikationsstudie handelte es sich um Studierende verschiedener Fachrichtungen an der Georg-August-Universität Göttingen. Der Stichprobenumfang wurde im Vergleich zur Originalstudie vergrößert (358 Probanden gegenüber 166; davon 178 Probanden in den $\mathrm{SC}^{(+)}$-Replikationsbedingungen), um eine höhere 
Teststärke zu erreichen und der Einführung einer ergänzenden $\mathrm{SC}^{(-)}$-Faktorstufe Rechnung zu tragen. Unter den Teilnehmern der Gesamtstichprobe waren 244 (68\%) weiblich und 114 (32\%) männlich. Das Durchschnittsalter betrug 22,19 Jahre $(S D=3.03)$, in einer Spanne zwischen 18 und 42 Jahren. $^{31}$

Das Versuchsdesign von Wong et al. (2008) wurde in dieser Replikationsstudie vollständig übernommen und um eine $\mathrm{SC}^{(-)}$-Faktorstufe erweitert. Hierdurch ergab sich also ein 2 (versunkene Kosten: vorhanden vs. nicht vorhanden) $\times 2$ (Framing: positiv vs. negativ) Zwischensubjektdesign, wobei das Framing wie in der Originalstudie lediglich als Kontrollvariable behandelt wurde. Der Rational Thinking Style als Prädiktor und die prozentuale Investitionsbereitschaft als Kriteriumsvariable wurden ebenfalls mit den gleichen Materialien wie in der Originalstudie erhoben.

\subsubsection{Durchführung und Material}

Die Untersuchung wurde, wie bei Wong et al. (2008), in Form einer papierbasierten Fragebogenstudie im Labor durchgeführt. Die Akquise der Probanden erfolgte über Aushänge im Universitätsgebäude sowie Direktansprachen durch studentische Hilfskräfte, welche auf dem Universitätsgelände auf die parallel laufende Erhebung hinwiesen. Es wurde mit einer begrenzten Anzahl von Schokoladentafeln als Probandenvergütung geworben. Alle Interessenten mussten für die Teilnahme an der Befragung selbständig einen bestimmten Hörsaal aufsuchen. Da es sich hierbei um den größten Hörsaal der Universität mit ungefähr 900 Plätzen handelte, war es nicht notwendig, eine Obergrenze gleichzeitiger Teilnehmer festzulegen. Jeder Proband konnte direkt nach seinem Eintreffen im Hörsaal an dieser fortlaufenden Erhebung teilnehmen.

\footnotetext{
${ }^{31}$ Unter den 178 Teilnehmern der reinen $\mathrm{SC}^{(+)}$-Replikationsbedingungen befanden sich 123 weibliche (69\%) und 55 männliche (31\%) Probanden. Das Durchschnittsalter betrug in dieser Gruppe 22,22 Jahre $(S D=3.22)$, in einer Spanne zwischen 18 und 42 Jahren. Es mussten keine Ausschlüsse vorgenommen werden.
} 
An den Eingängen des Hörsaals wies je ein Plakat auf den genauen Ablauf der Befragung hin. Auf diesem Wege wurden die Teilnehmer zunächst gebeten, direkt nach dem Eintreten einen Fragebogen bei der Versuchsleitung abzuholen, sich anschließend einen Platz im Hörsaal auszusuchen und ihren Fragebogen auszufüllen. Es wurde auch darauf hingewiesen, dass Ablenkungsmittel und Unterhaltungen nicht gestattet seien. Am Ende der Befragung sollten die Teilnehmer ihre Fragebögen selbständig wieder bei der Versuchsleitung abgeben um im Gegenzug ihre Versuchspersonenvergütung zu erhalten.

Die Reihenfolge der ausgegebenen Fragebögen wurde bereits im Vorfeld zufällig gemischt, sodass die Zuteilung der Probanden auf die Versuchsbedingungen beim Austeilen randomisiert erfolgte. Insgesamt umfasste ein Fragebogen vier Seiten. Auf der ersten Seite wurden das Geschlecht, das Alter, das Studienfach und die Semesteranzahl erhoben. Schriftliche Instruktionen erläuterten auf derselben Seite, dass es sich um Fragebögen zu den Themen „Persönlichkeitsmessung und Entscheidungsverhalten“ handele. Dabei wurde auch nochmal auf die klausurähnlichen Bedingungen bei der Bearbeitung zur Gewährleistung eines ruhigen Ablaufs sowie auf die Anonymität aller erhobenen Daten hingewiesen. Außerdem wurde die dreiteilige Struktur der Fragebögen vorgestellt, mit der Messung der Persönlichkeit im ersten Teil, der Erhebung des Entscheidungsverhaltens im zweiten Teil und einem Abschlussfragebogen im dritten Teil.

Die Persönlichkeitsmessung erfolgte auf der zweiten Seite. ${ }^{32}$ Hierbei wurde der Rational Thinking Style auf Trait-Ebene durch die gleiche Rationalitätsskala erhoben, die auch bei Wong et al. (2008) verwendet wurde (vgl. Pacini \& Epstein, 1999). Die Skala umfasst

\footnotetext{
32 Anders als in der Originalstudie, wurde es im Rahmen der vorliegenden Arbeit als methodisch besser betrachtet, zuerst die Trait-Ausprägung des Rational Thinking Styles und erst danach die Investitionsbereitschaft als abhängige Variable zu erheben. Um die Vergleichbarkeit zur Originalstudie nicht in Frage zu stellen, wurde eine Vorstudie mit 80 Teilnehmern durchgeführt, in der jeweils zur Hälfte zuerst der Rationalitätsfragebogen oder das Sunk-Cost-Szenario präsentiert wurde. Dabei zeigten sich durchweg keine Haupt- oder Interaktionseffekte für die Reihenfolge, sodass es gerechtfertigt erschien, die vorliegende Replikationsstudie in der oben beschriebenen Reihenfolge durchzuführen. Eine detailliertere Beschreibung sowie Analysen hierzu finden sich in Anhang B.
} 
insgesamt 20 Items, welche zuvor ins Deutsche übersetzt wurden. Von allen Items beziehen sich jeweils zehn auf die Subskalen „Rational Ability (RA)“ und „Rational Engagement (RE)“ (vgl. Abschnitt 2.2.2.4, siehe Anhang A). Die Fragen waren entweder positiv oder negativ formuliert (Beispiele: $\mathrm{RA}^{(+)}$: „Ich habe einen logischen Verstand“, $\mathrm{RA}^{(-)}$: „Ich bin kein besonders analytischer Denker“; $\mathrm{RE}^{(+)}$: „Ich genieße intellektuelle Herausforderungen“, $\mathrm{RE}^{(-)}$: „Die Antwort zu kennen, ohne den Grund dahinter verstehen zu müssen, ist ausreichend für mich"). Ihre Zustimmung zu den jeweiligen Aussagen gaben die Probanden auf einer fünfstufigen Skala mit den Endpunkten „,trifft überhaupt nicht zu“ (1) und „trifft voll und ganz $\mathrm{zu}^{\prime \prime}(5)$ an.

Auf der dritten Seite erfolgte die Messung der prozentualen Investitionsbereitschaft mithilfe des von Wong et al. (2008) verwendeten Sunk-Cost-Szenarios. Das Szenario wurde für die vorliegenden Zwecke ins Deutsche übersetzt und um eine Version ohne versunkene Kosten ergänzt (siehe Anhang A). Inhaltlich handelte es sich wie erwähnt um eine Variante des Airplane-Szenarios. Darin wird anstelle eines Tarnflugzeugs ein Tarnschiff entwickelt. Die versunkenen Kosten betragen 5 Millionen Euro, aus einem Budget von insgesamt 10 Millionen Euro. Die im Rahmen der Replikationsstudie neu geschaffene Version ohne versunkene Kosten wurde so nahe wie möglich an Arkes und Blumers (1985) AirplaneSzenario angelehnt, also erhielten die Probanden hierin einen Vorschlag eines Angestellten, eine Million Euro in ein entsprechendes Forschungsprojekt zu investieren.

Über das ursprüngliche Airplane-Szenario hinausgehend, wurden analog zu Wong et al. (2008) auch zukünftige Gewinnmöglichkeiten angegeben. Demnach könnten mit dem Projekt 600 Millionen Euro verdient werden. Diese Angabe variierte im Zuge der inter-individuellen Manipulation des positiven vs. negativen Framings, indem entweder der potenzielle Gewinn (positives Framing) oder der potenzielle Verlust (negatives Framing) in den Vordergrund gestellt wurde. Wie bereits erwähnt wurde das Framing in der Originalstudie als Kontrollvariable behandelt, da es laut Wong et al. (2008) eigentlich einem anderen 
Forschungsvorhaben dienen sollte und irrelevant für ihre berichtete Studie war. Im Rahmen der vorliegenden Arbeit wurde Framing also nur der Vollständigkeit halber in das Design mit aufgenommen, weswegen auf eine ausführliche Behandlung dieses Faktors im Folgenden verzichtet wird. Durch die Kombination der zweistufigen Faktoren „versunkene Kosten“ und „Framing“ ergaben sich in der Replikationsstudie insgesamt vier verschiedene Fragebogenversionen. Wie beschrieben wurde die Reihenfolge der Fragebögen zuvor gemischt, wodurch eine randomisierte Aufteilung der Probanden auf eine der vier Versuchsbedingungen im Rahmen der Austeilung gewährleistet wurde.

Auf der letzten Fragebogenseite folgte ein Abschlussfragebogen. Hierauf wurde wie in der Originalstudie das subjektive Verantwortlichkeitsempfinden als Kontrollvariable durch zwei Items erhoben, die Wong et al. (2008) von Conlon und Parks (1987) adaptiert hatten. Die beiden Fragen zielten auf das Verantwortungsempfinden für die initiale Investition sowie den Projektstart ab. Während beide Items auf Stufe vorhandener versunkener Kosten ohne Probleme erhoben werden konnten, war dieses auf der Stufe ohne versunkene Kosten inhaltlich nicht möglich. Schließlich gab es in der $\mathrm{SC}^{(-)}$-Version des Szenarios weder eine initiale Investition noch ein bereits gestartetes ein Projekt. Aus diesem Grunde wurden diese Items ausschließlich in den $\mathrm{SC}^{(+)}$-Replikationsbedingungen abgefragt. Als vergleichbares Verantwortlichkeits-Item wurde eine dritte Frage neu entwickelt, die auf beiden Sunk-CostStufen verwendet werden konnte. Sie lautete: „Wie verantwortlich fühlst Du Dich für den Erfolg der getroffenen Entscheidung?““. Alle drei Verantwortlichkeits-Items wurden auf einer siebenstufigen Skala mit den Endpunkten „sehr wenig“ (1) und „sehr stark“ (7) erhoben.

Anschließend sollten die Probanden angeben, ob sie bereits in der Vergangenheit an einer ähnlichen Studie teilgenommen haben, worum es darin inhaltlich ging und wie diese ablief. Im Sinne einer Verdachtskontrolle sollten die Probanden außerdem schildern, wonach es ihrer Meinung nach in der vorliegenden Untersuchung ging. Um nach Abschluss aller Erhebungen über die Hintergründe der Replikationsstudie aufgeklärt zu werden, wurden die Probanden 
gebeten, ihre E-Mail-Adresse anzugeben. An dieser Stelle war die Befragung abgeschlossen und den Probanden wurde mit Bitte um Verschwiegenheit noch einmal schriftlich für die Teilnahme gedankt.

Entsprechend der Instruktionen des Plakats, welches die Teilnehmer zu Beginn der Befragung an den Eingängen des Hörsaals empfing, gaben sie ihre ausgefüllten Fragebögen selbständig bei der Versuchsleitung ab und erhielten im Gegenzug die versprochene Tafel Schokolade als Teilnahmevergütung. Der Erhalt der Schokolade wurde auf einer TeilnehmerQuittungsliste protokolliert, um unter anderem Mehrfachteilnahmen entgegenzuwirken. Aus demselben Grund wurde auch eine konstante Versuchsleitung eingesetzt, die jeden Probanden mit Dank persönlich verabschiedete. Die Versuchsleitung achtete darauf, dass die gesamte Phase nach der Fragebogenabgabe so leise wie möglich ablief, um die parallelen Erhebungen nicht zu stören. Eine Aufklärung über die Hintergründe dieser Untersuchung erfolgte nach Abschluss aller Erhebungen per E-Mail.

\subsubsection{Ergebnisse}

Wie in der Originalstudie wurde als Kriteriumsvariable die „prozentuale Investitionsbereitschaft" erhoben, welche die Probanden in einer Spanne zwischen 0 (absolut nicht bereit) und 100 Prozent (vollkommen bereit) in ein separates Feld eintrugen. Die versunkenen Kosten wurden mit „,“ $\left(\mathrm{SC}^{(-)}\right)$und „1“ $\left(\mathrm{SC}^{(+)}\right)$codiert, ebenso das Framing (,,0“ = negativ, „1“ = positiv). Vor der Berechnung der Rationalitätsskalen wurden zunächst alle negativ formulierten Items invertiert (vgl. Pacini \& Epstein, 1999, S. 976). Anschließend wurden die Skalen „Rational Thinking Style“, „Rational Ability“ und „Rational Engagement“ wie bei Wong et al. (2008) durch den Mittelwert der zugehörigen Items gebildet. Die Chronbach's Alpha Werte der drei Skalen betrugen in der Replikationsstudie für RTS $=.83$, für $\mathrm{RA}=.75$ und für $\mathrm{RE}=.74$ und waren somit sehr gut vergleichbar zu den Werten aus der Originalstudie $(\mathrm{RTS}=.84, \mathrm{RA}=.78, \mathrm{RE}=.64)$. 
Die Berichterstattung der Ergebnisse erfolgt auch für die vorliegende Replikationsstudie in zwei Schritten, wie bereits in den Replikationsstudien zum regulatorischen Fokus. Zunächst wird eine an die Originalstudie angelehnte Analyse der reinen $\mathrm{SC}^{(+)}$-Replikationsbedingungen berichtet, um eine bestmögliche Vergleichbarkeit zu den Befunden von Wong et al. (2008) zu gewährleisten. Im Anschluss folgt eine umfassendere Analyse des um eine SC ${ }^{(-)}$-Faktorstufe erweiterten Designs der vorliegenden Replikationsstudie, um potenzielle Interaktionseffekte aufdecken zu können.

\subsubsection{Analyse der reinen Replikationsbedingungen}

Tabelle 9 enthält zunächst die Mittelwerte, Standardabweichungen und Korrelationskoeffizienten der Variablen aus der Originalstudie. Für einen direkten Vergleich sind die entsprechenden Ergebnisse auf Stufe vorhandener versunkener Kosten der Replikationsstudie in Tabelle 10 aufgelistet.

Tabelle 9: Mittelwerte, Standardabweichungen und Korrelationen der Variablen bei Wong et al. (2008).

\begin{tabular}{|c|c|c|c|c|c|c|c|c|c|}
\hline & M & SD & 1 & 2 & 3 & 4 & 5 & 6 & 7 \\
\hline 1. Probability of Investment & 61.10 & 26.80 & --- & & & & & & \\
\hline 2. Rational Thinking Style & 4.64 & .76 & $.14^{\dagger}$ & --- & & & & & \\
\hline 3. Rational Ability & 4.58 & .93 & $.17^{*}$ & $.92^{* *}$ & --- & & & & \\
\hline 4. Rational Engagement & 4.70 & .76 & .08 & $.88^{* *}$ & $.63^{* *}$ & --- & & & \\
\hline 5. Personal Responsibility & 5.03 & .89 & $.27^{* *}$ & -.02 & -.04 & .00 & --- & & \\
\hline 6. Age & 20.49 & 1.30 & $.17^{*}$ & $.16^{*}$ & $.13^{\dagger}$ & $.16^{*}$ & .03 & --- & \\
\hline $\begin{array}{l}\text { 7. Gender } \\
(0=\text { female, } 1=\text { male })\end{array}$ & .63 & .49 & .08 & $.25^{* *}$ & $.25^{* *}$ & $.20^{*}$ & .02 & .05 & --- \\
\hline $\begin{array}{l}\text { 8. Framing condition } \\
(0=\text { negative, } 1=\text { positive })\end{array}$ & .51 & .50 & $-.28^{* * *}$ & $.16^{*}$ & $.16^{*}$ & .12 & $-.22^{* *}$ & $-.22^{* *}$ & -.07 \\
\hline
\end{tabular}

Anmerkung: $\dagger p<.05$, einseitig; $* p<.05$, zweiseitig; $* * p<.01$, zweiseitig; $* * * p<.001$, zweiseitig.

In der Replikationsstudie fanden sich ähnlich hohe signifikante Korrelationen der drei Rationalitätsskalen untereinander wie in der Originalstudie. Im Gegensatz zur Originalstudie blieben signifikante Korrelationen der Skalen RTS und RA mit der Investitionsbereitschaft hingegen aus. Die entsprechenden Korrelationskoeffizienten der Replikationsstudie waren extrem niedrig und nicht signifikant (RTS: $r=-.03, p=.653$; RA: $r=-.04, p=.595$ ). Eine 
signifikante Korrelation zwischen RE und der Investitionsbereitschaft zeigte sich in keiner der beiden Studien.

Tabelle 10: Mittelwerte, Standardabweichungen und Korrelationen der Variablen in der Replikationsstudie.

\begin{tabular}{|c|c|c|c|c|c|c|c|c|c|}
\hline & M & SD & 1 & 2 & 3 & 4 & 5 & 6 & 7 \\
\hline 1. Probability of Investment & 51.07 & 28.45 & --- & & & & & & \\
\hline 2. Rational Thinking Style & 3.55 & .46 & -.03 & --- & & & & & \\
\hline 3. Rational Ability & 3.56 & .55 & -.04 & $.89^{* * *}$ & --- & & & & \\
\hline 4. Rational Engagement & 3.53 & .50 & -.02 & $.86^{* * *}$ & $.54^{* * *}$ & --- & & & \\
\hline 5. Personal Responsibility & 4.97 & 1.35 & $.15^{*}$ & -.07 & $-.13^{\dagger}$ & .01 & --- & & \\
\hline 6. Age & 22.22 & 3.22 & -.10 & .11 & $.15^{*}$ & .04 & -.09 & --- & \\
\hline $\begin{array}{l}\text { 7. Gender } \\
(0=\text { female }, 1=\text { male })\end{array}$ & .31 & .46 & -.10 & $.13^{\dagger}$ & $.16^{*}$ & .05 & $-.20^{* *}$ & .12 & --- \\
\hline $\begin{array}{l}\text { 8. Framing condition } \\
(0=\text { negative, } 1=\text { positive })\end{array}$ & .50 & .50 & $-.22^{* *}$ & -.04 & -.03 & -.03 & $-.13^{\dagger}$ & .06 & -.06 \\
\hline
\end{tabular}

Anmerkung: $\dagger p<.05$, einseitig; $* p<.05$, zweiseitig; $* * p<.01$, zweiseitig; $* * * p<.001$, zweiseitig.

Die Ergebnisse der hierarchischen Regressionsanalysen aus der Originalstudie und der Replikationsstudie werden in Tabelle 11 gegenübergestellt. Modell 1a analysiert den Einfluss der RTS Gesamtskala auf die Investitionsbereitschaft und Modell 1b den Einfluss der beiden Subskalen RA und RE, jeweils unter Berücksichtigung aller Kontrollvariablen.

Tabelle 11: Resultate der hierarchischen Regressionsanalysen von Wong et al. (2008) und der Replikationsstudie im Vergleich. Dargestellt werden die standardisierten Beta-Koeffizienten, die Kriteriumsvariable ist jeweils die prozentuale Investitionsbereitschaft.

\begin{tabular}{|c|c|c|c|c|c|c|}
\hline \multirow[b]{2}{*}{ Variables } & \multicolumn{3}{|l|}{ Original } & \multicolumn{3}{|c|}{ Replication } \\
\hline & $\begin{array}{c}\text { Model } \\
1\end{array}$ & $\begin{array}{c}\text { Model } \\
\text { la }\end{array}$ & $\begin{array}{c}\text { Model } \\
1 b\end{array}$ & $\begin{array}{c}\text { Model } \\
1\end{array}$ & $\begin{array}{c}\text { Model } \\
\text { la }\end{array}$ & $\begin{array}{c}\text { Model } \\
1 b\end{array}$ \\
\hline \multicolumn{7}{|l|}{ Control Variables } \\
\hline Personal Responsibility & $.24^{* *}$ & $.24^{* *}$ & $.24^{* * *}$ & .10 & .10 & .10 \\
\hline Age & $.13^{\dagger}$ & .09 & .10 & -.07 & -.07 & -.07 \\
\hline Gender & .05 & .01 & .01 & -.08 & -.08 & -.08 \\
\hline Framing & $-.19^{*}$ & $-.22^{* *}$ & $-.23^{* *}$ & $-.22^{* *}$ & $-.22^{* *}$ & $-.22^{* *}$ \\
\hline \multicolumn{7}{|l|}{ Study Variables } \\
\hline Rational Thinking Style & & $.17^{*}$ & & & -.02 & \\
\hline Rational Ability & & & $.24^{* *}$ & & & .01 \\
\hline Rational Engagement & & & -.06 & & & -.03 \\
\hline$\Delta R^{2}$ & & .02 & .04 & & .00 & .00 \\
\hline$F$ change & & $4.58^{*}$ & $3.67^{*}$ & & .06 & .05 \\
\hline Overall model $R^{2}$ & .15 & .17 & .18 & .08 & .08 & .08 \\
\hline Adjusted $R^{2}$ & .12 & .14 & .15 & .06 & .06 & .05 \\
\hline Overall model $F$ & $6.78^{* * *}$ & $6.46^{* * * *}$ & $5.89^{* * *}$ & $3.81^{* *}$ & $3.05^{*}$ & $2.53^{*}$ \\
\hline
\end{tabular}

Anmerkung: $\dagger p<.05$, einseitig; $* p<.05$, zweiseitig; $* * p<.01$, zweiseitig; $* * * p<.001$, zweiseitig 
Die zentralen Befunde der Originalstudie waren, dass sich sowohl für den RTS als auch für RA ein positiver, signifikanter Einfluss auf die Investitionsbereitschaft zeigte. Personen mit hohen Rationalitätsausprägungen auf diesen beiden Skalen wiesen demnach eine höhere Investitionsbereitschaft auf als Personen mit niedrigen Ausprägungen. In der vorliegenden Replikationsstudie war hingegen kein Einfluss der beiden Rationalitätsskalen auf die Investitionsbereitschaft nachweisbar (RTS: $\beta=-.02, t[171]=-.24, p=.811 ; \mathrm{RA}: \beta=.01$, $t[172]=.06, p=.956)$. Für RE fand sich in Übereinstimmung mit der Originalstudie ebenfalls kein Effekt $(\beta=-.03, t[172]=-.29 p=.772)$.

Davon abgesehen konnten auch die in der Originalstudie berichteten Effekte für die Kontrollvariable „Verantwortlichkeit“ nicht repliziert werden, welche in der vorliegenden Analyse aus den beiden Originalitems gebildet wurde. Anders verhielt es sich mit „Framing“. Für diesen Faktor fanden sich in der Originalstudie zwar signifikante Effekte, die in vergleichbarer Form auch in der Replikationsstudie auftraten, jedoch wurden die Originalbefunde aufgrund der erläuterten Irrelevanz des Faktors nicht von den Autoren diskutiert. Aus diesem Grund enthält sich auch die vorliegende Arbeit einer Diskussion dieser Befunde. Unabhängig davon bleibt festzuhalten, dass sich für die zentralen Befunde der Originalstudie in den reinen $\mathrm{SC}^{(+)}$-Replikationsbedingungen der Replikationsstudie keine Anzeichen fanden.

\subsubsection{Analyse des erweiterten Versuchsdesigns}

Weitere Analysen, unter Einschluss der ergänzten $\mathrm{SC}^{(-)}$-Bedingungen, sollten Aufschluss darüber geben, inwiefern der RTS und seine Subskalen RA und RE als Moderator für den Sunk-Cost-Effekt betrachtet werden können.

Zunächst werden in Tabelle 12 die Mittelwerte, Standardabweichungen und Korrelationskoeffizienten der Variablen aus der Replikationsstudie für das erweiterte Design aufgelistet. 
Tabelle 12: Mittelwerte, Standardabweichungen und Korrelationen der Variablen im erweiterten Design der Replikationsstudie.

\begin{tabular}{|c|c|c|c|c|c|c|c|c|c|}
\hline & M & SD & 1 & 2 & 3 & 4 & 5 & 6 & 7 \\
\hline 1. Probability of Investment & 46.13 & 28.95 & --- & & & & & & \\
\hline 2. Rational Thinking Style & 3.56 & .48 & -.02 & --- & & & & & \\
\hline 3. Rational Ability & 3.57 & .55 & -.04 & $.88^{* * * *}$ & --- & & & & \\
\hline 4. Rational Engagement & 3.56 & .54 & .01 & $.87^{* * * *}$ & $.53^{* * *}$ & --- & & & \\
\hline $\begin{array}{l}\text { 5. Sunk Cost } \\
(0=S C-, 1=S C+)\end{array}$ & .05 & .05 & $.17^{* *}$ & -.03 & -.01 & -.05 & --- & & \\
\hline 6. Age & 22.19 & 3.03 & -.08 & $.13^{*}$ & $.17^{* *}$ & .06 & .01 & --- & \\
\hline $\begin{array}{l}\text { 7. Gender } \\
(0=\text { female, } 1=\text { male })\end{array}$ & .32 & .47 & .00 & $.12^{*}$ & $.16^{* *}$ & .06 & -.02 & $.09^{\dagger}$ & --- \\
\hline $\begin{array}{l}\text { 8. Framing condition } \\
(0=\text { negative, } 1=\text { positive })\end{array}$ & .50 & .50 & -28 & -.08 & -.05 & -.08 & -.01 & .01 & $-.09^{\dagger}$ \\
\hline
\end{tabular}

Anmerkung: $\dagger p<.05$, einseitig; $* p<.05$, zweiseitig; $* * p<.01$, zweiseitig; $* * * p<.001$, zweiseitig.

Es bestätigten sich die signifikanten Korrelationen der drei Rationalitätsskalen untereinander in vergleichbarer Höhe, wie in der Originalstudie und bei der Betrachtung der reinen $\mathrm{SC}^{(+)}$-Replikationsbedingungen zuvor. Signifikante Korrelationen dieser Skalen mit der Investitionsbereitschaft waren allerdings auch hier nicht feststellbar (RTS: $r=-.02, p=.709$, RA: $r=-.04, p=.413$, RE: $r=.01, p=.884)$. In dieser erweiterten Betrachtung zeigte sich darüber hinaus eine signifikante, positive Korrelation zwischen den versunkenen Kosten und der Investitionsbereitschaft.

Tabelle 13 veranschaulicht die Ergebnisse der hierarchischen Regressionsanalysen innerhalb des erweiterten Designs der vorliegenden Replikationsstudie. Analog zum vorherigen Abschnitt, bezieht sich Modell 2a wieder auf den Einfluss der RTS Gesamtskala auf die Investitionsbereitschaft und Modell 2b den Einfluss der beiden Subskalen RA und RE, jeweils unter Berücksichtigung der Kontrollvariablen. ${ }^{33}$

33 Eine Bestimmung des persönlichen Verantwortlichkeitsempfindens anhand der Originalitems war, wie erwähnt, in den $\mathrm{SC}^{(-)}$-Bedingungen nicht möglich. Das neu entwickelte Item, zur Abfrage des Verantwortlichkeitsempfindens für den Erfolg, wurde allerdings von vielen Versuchspersonen versehentlich missachtet und aus diesem Grunde nicht in die vorliegende Analyse mit einbezogen. Ein Einbezug des Studienfaches und der Semesterzahl, als zusätzliche Kontrollvariablen, änderte übrigens nichts an der zentralen Befundlage aller in diesem Unterkapitel berichteten Analysen. 
Tabelle 13: Resultate der hierarchischen Regressionsanalysen des erweiterten Designs der Replikationsstudie. Dargestellt werden die standardisierten Beta-Koeffizienten, die Kriteriumsvariable ist die prozentuale Investitionsbereitschaft.

\begin{tabular}{|c|c|c|c|}
\hline \multirow[b]{2}{*}{ Variables } & \multicolumn{3}{|c|}{ Replication (advanced design) } \\
\hline & Model 2 & Model $2 a$ & Model $2 b$ \\
\hline \multicolumn{4}{|l|}{ Control Variables } \\
\hline Age & -.08 & -.08 & -.07 \\
\hline Gender & -.01 & -.01 & -.01 \\
\hline Framing & $-.28^{* * *}$ & $-.28^{* * *}$ & $-.28^{* * *}$ \\
\hline \multicolumn{4}{|l|}{ Study Variables } \\
\hline Rational Thinking Style & & -.01 & \\
\hline Rational Ability & & & -.08 \\
\hline Rational Engagement & & & .06 \\
\hline Sunk Cost & $.17^{* *}$ & .25 & .22 \\
\hline Interaction Sunk Cost $*$ RTS & & -.08 & \\
\hline Interaction Sunk Cost $*$ RA & & & .16 \\
\hline Interaction Sunk Cost $* \mathrm{RE}$ & & & -.21 \\
\hline$\Delta R^{2}$ & & .00 & .00 \\
\hline$F$ change & & .13 & .30 \\
\hline Overall model $R^{2}$ & .12 & .12 & .12 \\
\hline Adjusted $R^{2}$ & .11 & .10 & .10 \\
\hline Overall model $F$ & $11.47^{* * *}$ & $7.65^{* * *}$ & $5.84^{* * * *}$ \\
\hline
\end{tabular}

Anmerkung: $\dagger p<.05$, einseitig; $* p<.05$, zweiseitig; $* * p<.01$, zweiseitig; $* * * p<.001$, zweiseitig

In den Regressionsanalysen dieses erweiterten Designs fanden sich hinsichtlich der zentralen Fragestellung keine Hinweise auf eine Interaktion zwischen den versunkenen Kosten und den drei Rationalitätsskalen (RTS*SC: $\beta=-.08, t[351]=-.21, p=.835, r=.16$; $\mathrm{RA} * \mathrm{SC}: \beta=.16, t[349]=.40, p=.691, r=.16 ; \mathrm{RE}^{*} \mathrm{SC}: \beta=-.21, t[349]=-.52, p=.604, r=$ .16). Von einem moderierenden Einfluss des Rational Thinking Styles auf den Sunk-CostEffekt kann auf Basis der vorliegenden Ergebnisse somit nicht ausgegangen werden.

In der Berechnung des Modells 2, also ohne Einbezug der Rationalitätsskalen und möglichen Interaktionen, zeigte sich ein signifikanter Sunk-Cost-Effekt $(\beta=.17, t[353]=.17$, $p=.001)$ in der Form, dass die Probanden bei hohen versunkenen Kosten eine signifikant höhere Investitionsbereitschaft aufwiesen als in Abwesenheit versunkener Kosten $\left(M_{(S C+)}=\right.$ $51,1 \%$, vs. $\left.M_{(S C-)}=41,3 \%\right)$. Dieser Effekt verschwand jedoch in den weiteren Analysen 2a und $2 b$, in denen eine größere Anzahl von Variablen in das Modell mit aufgenommen wurde. 
Darüber hinaus konnten die Haupteffekte der Originalstudie hinsichtlich der Rationalitätsskalen, wonach höhere Rationalitätsausprägungen für RTS und RA mit einer höheren Investitionsbereitschaft einhergingen, auch in dieser erweiterten Analyse nicht repliziert werden $(\mathrm{RTS}: \beta=-.01, t[351]=-.21, p=.836 ; \mathrm{RA}: \beta=-.08, t[349]=-.94, p=.348$; RE: $\beta=.06, t[349]=.71, p=.480)$.

\subsubsection{Diskussion}

Die vorliegende Untersuchung stellte einen direkten Replikationsversuch zu Wong et al. (2008, Studie 1) dar. Die Autoren berichteten eine stärkere Investitionsbereitschaft für Personen mit hohen Trait-Rationalitätswerten als für Personen mit niedrigen Werten. Dieses Ergebnis war vor allem auf die Fähigkeitskomponente zum rationalen Denken zurückzuführen, statt auf die motivationale Komponente. Zur Messung der Investitionsbereitschaft wurde in der Originalstudie eine modifizierte Version von Arkes und Blumers (1985) Airplane-Szenarios eingesetzt, jedoch ohne dabei die versunkenen Kosten zu variieren.

In der Replikationsstudie wurde ein erweitertes Design verwendet, welches zwei Stufen der versunkenen Kosten umfasste. Der hierin geglückte Nachweis eines signifikanten SunkCost-Effekts kann als Indiz dafür angesehen werden, dass die Integration der zweiten Faktorstufe - durch die neu geschaffene Szenario-Version ohne versunkene Kosten - in der Replikationsstudie erfolgreich war. Leider zeigten sich darauf aufbauend aber keinerlei signifikante Interaktionseffekte zwischen den versunkenen Kosten und dem RTS bzw. seinen Subskalen RA und RE. Diese Befundlage spricht daher gegen die Annahme, dass es sich bei dem Rational Thinking Style um eine Moderatorvariable für den Sunk-Cost-Effekt handeln könnte.

Ferner konnten auch die grundlegenden Effekte von Wong et al. (2008) nicht repliziert werden, bei denen höhere Ausprägungen des Rational Thinking Styles und der Rational 
Ability mit einer höheren Investitionsbereitschaft einhergingen als niedrige Ausprägungen.

Die Korrelationskoeffizienten der jeweiligen Rationalitätsskalen mit der Investitionsbereitschaft tendierten in der näherungsweise teststärkeren Replikationsstudie sogar gegen Null.

Insgesamt lassen die Ergebnisse der Replikationsstudie stark daran zweifeln, dass tatsächlich ein Zusammenhang zwischen dem Rational Thinking Style und der Investitionsbereitschaft bestehen könnte. Ebenso zweifelhaft erscheint die Annahme, dass es sich bei dem Rational Thinking Style um eine Moderatorvariable für den Sunk-Cost-Effekt handeln könnte. Dennoch wird an dieser Stelle auf ein abschließendes Urteil verzichtet und stattdessen auf die in Kapitel 5 berichtete integrative Replikationsstudie verwiesen, die einen weiteren Replikationsversuch der Effekte des Rational Thinking Styles auf Trait-Ebene darstellt. 


\subsection{Fazit der direkten Replikationsstudien}

In diesem Kapitel wurden alle direkten Replikationsstudien vorgestellt, die im Rahmen der vorliegenden Arbeit durchgeführt worden sind. Im Sinne einer exakten Replikation wurde jeweils versucht, alle Aspekte der entsprechenden Originalstudien bestmöglich nachzustellen. Zusätzlich wurden mindestens gleich große Stichprobenumfänge wie in den Originalstudien gewählt, in den meisten Fällen sogar größere. Wie eingangs erläutert, kann so von näherungsweise höheren Teststärken der Replikationsstudien gegenüber den Originalstudien ausgegangen werden. Größere Teststärken erhöhen in der Regel die Sensitivität, tatsächlich existierende Effekte in den entsprechenden Tests statistisch nachweisen zu können (vgl. Asendorpf et al., 2013; Bortz \& Schuster, 2010; Cohen, 1962). Trotzdem konnten, anders als erwartet, in keiner der direkten Replikationsstudien die zentralen Befunde der jeweiligen Originalstudien repliziert werden:

Bei der Handlungs- und Lageorientierung (van Putten et al., 2010) auf Trait-Ebene blieb der Nachweis einer signifikanten Interaktion zwischen dem Persönlichkeitskonstrukt und den versunkenen Kosten aus. Während sich die handlungsorientierten Probanden in der Originalstudie vergleichsweise indifferent hinsichtlich der versunkenen Kosten verhielten und damit eine gewisse Robustheit gegenüber dem Sunk-Cost-Effekt aufwiesen, waren im Gegensatz dazu signifikante Sunk-Cost-Effekte sowohl für Lageorientierung als auch für Handlungsorientierung in der Replikationsstudie nachweisbar. In Übereinstimmung mit Arkes und Blumers (1985) grundlegenden Befund wiesen die Sunk-Cost-Effekte jeweils positive Vorzeichen auf, während die Befunde der Originalstudie mit negativen Vorzeichen in die genau gegensätzliche Richtung wiesen. Leider ist aufgrund einer nicht beantworteten Anfrage an die Erstautorin der Originalarbeit zum derzeitigen Zeitpunkt ungeklärt, ob es sich in diesem Fall um eine deutliche Nichtreplikation handelt oder ob bei van Putten et al. (2010) ein Vorzeichenfehler vorgelegen haben könnte. Unabhängig davon gilt aber, dass der in der 
Originalstudie berichtete Einfluss der Handlungs- und Lageorientierung nicht repliziert werden konnte.

Im Falle von zustandsbezogenem Optimismus und Pessimismus (Juliusson, 2006) wurden zwei direkte Replikationsstudien auf State-Ebene durchgeführt, deren Stichprobenumfänge im Vergleich zur Originalstudie nahezu verdoppelt wurden. Der Autor berichtete eine gesteigerte Investitionsneigung bei Optimisten und eine gesteigerte Abbruchneigung bei Pessimisten, wobei beide Neigungen durch hohe gegenüber niedrigen versunkenen Kosten jeweils noch verstärkt wurden. Dieses konnte in beiden Replikationsstudien allerdings nicht bestätigt werden, da der entsprechende Interaktionseffekt zwischen dem Persönlichkeitskonstrukt und den versunkenen Kosten nicht replizierbar war. Rein deskriptiv kehrte sich das beschriebene Verhaltensmuster aus der Originalstudie in der ersten Replikationsstudie sogar um und ging in der zweiten Replikationsstudie eher in Richtung eines generellen Effekts.

In Bezug auf den regulatorischen Fokus: Promotion und Prevention (Molden \& Hui, 2011) auf State-Ebene, konnten zwei direkte Replikationsstudien die zentrale Aussage der Autoren, dass ein Promotion Focus hilfreich sei, um versunkene Kosten besser zu ignorieren, ebenfalls nicht stützen. In beiden Replikationsstudien, die jeweils einen höheren Stichprobenumfang als die Originalstudie besaßen, waren keine Interaktionseffekte zwischen dem regulatorischen Fokus und den versunkenen Kosten nachweisbar. Darüber hinaus war auch der grundlegende Befund aus der Originalstudie nicht replizierbar, in der Probanden mit einem Promotion Focus die niedrigste Investitionsrate aller Vergleichsgruppen aufwiesen. In der ersten Replikationsstudie war sogar genau das Gegenteil der Fall, während sich in der zweiten Replikationsstudie kein Unterschied zwischen den Faktorstufen fand.

Auch hinsichtlich des Rational Thinking Styles auf Trait Ebene war der direkte Replikationsversuch nicht erfolgreich. Im Gegensatz zur Originalstudie, in der hohe Werte der Skalen RTS und RA mit einer hohen Investitionsbereitschaft einhergingen, zeigten sich in der Replikationsstudie trotz größeren Stichprobenumfangs keinerlei Zusammenhänge. In dem 
über die Originalstudie hinausgehenden Design, mit zwei Faktorstufen der versunkenen Kosten, blieben die zentralen Interaktionseffekte zwischen den Rationalitätsskalen und den versunkenen Kosten ebenfalls aus. Daher kann auch in Bezug auf den Rational Thinking Style nicht von einer Moderatorvariable für den Sunk-Cost-Effekt ausgegangen werden.

Im Rahmen dieser Arbeit sollte nach Sicherstellung der Replizierbarkeit der vorgestellten Originalbefunde ursprünglich überprüft werden, ob die vier Persönlichkeitskonstrukte zu einer differenziellen Entscheidungsfindung befähigen, die sich durch die Abwesenheit von Sunk-Cost-Effekten auszeichnet, oder ob in den Originalstudien stattdessen nur die Investitionsneigung pauschal verändert wurde. Da aber alle direkten Replikationsversuche der Originalbefunde durchgehend gescheitert sind, stellt sich diese Frage aktuell nicht mehr. Stattdessen liegt die Vermutung nahe, dass es die in den Originalstudien berichteten Effekte unter Umständen gar nicht gibt. Aus diesem Grund wird im folgenden Kapitel direkt zu den Berichten der integrativen Replikationsstudie übergegangen, die einen weiteren Replikationsversuch der Originalbefunde im Rahmen der vorliegenden Arbeit darstellte (vgl. Kapitel 3). 


\section{Integrative Replikationsstudie}

Die im Folgenden berichtete integrative Replikationsstudie stellte einen weiteren, konzeptuellen Versuch dar, die Originalbefunde von van Putten et al. (2010), Juliusson (2006), Molden und Hui (2011) sowie Wong et al. (2008) im Rahmen der vorliegenden Arbeit zu replizieren. Im Gegensatz zu den direkten Replikationsstudien aus Kapitel 4, in denen die entsprechenden Persönlichkeitseffekte isoliert untersucht wurden, erfolgte in der integrativen Replikationsstudie eine simultane Erhebung der vier Persönlichkeitskonstrukte. Diese Vorgehensweise trägt der Tatsache Rechnung, dass das Entscheidungsverhalten von mehreren Persönlichkeitsvariablen gleichzeitig beeinflusst werden kann. Es wäre nämlich denkbar, dass die Prädiktoren miteinander interkorrelieren und bestimmte Persönlichkeitsvariablen ihren prädiktiven Wert einbüßen, sobald die jeweils anderen Variablen ebenfalls einen Einfluss ausüben. Unter Umständen ließen sich hierdurch sogar einige der berichteten Originaleffekte stattdessen auf die anderen Variablen zurückführen.

Um die simultane Erhebung aller Persönlichkeitsvariablen adäquat umsetzen zu können, erfolgten die entsprechenden Messungen ausschließlich auf Trait-Ebene. Die dichotome Investitionsentscheidung und die prozentuale Investitionsbereitschaft, die einzeln auch in den jeweiligen Originalarbeiten verwendet wurden, dienten beide als Kriteriumsvariablen. Die Sunk-Cost-Neigung wurde sowohl im Airplane-Szenario gemessen, welches in drei der vier Originalarbeiten zum Einsatz kam, als auch im Druckerpressen-Szenario von Arkes und Blumer (1985). Es wurden daher gleich zwei empirisch gut bewährte Sunk-Cost-Szenarien eingesetzt, um die Originalbefunde zu replizieren (vgl. Abschnitt 2.1.3). ${ }^{34}$

\footnotetext{
${ }^{34}$ Sofern sich Effekte aufdecken lassen, könnten durch den Einsatz der beiden verschiedenen Sunk-CostSzenarien auch näherungsweise Aussagen hinsichtlich einer differenziellen Entscheidungsfähigkeit getroffen werden.
} 


\subsection{Methode}

\subsubsection{Stichprobe und Design}

Die Teilnehmer der vorliegenden Untersuchung waren Studierende verschiedener Fachrichtungen der Georg-August-Universität Göttingen. Von den insgesamt 120 Probanden bearbeitete einer die Sunk-Cost-Szenarien gar nicht; ein weiterer investierte im AiplaneSzenario nicht, mit der Begründung, dass es sich seiner Meinung nach um die Finanzierung von Kriegsgerät handele. Diese beiden Personen wurden aus allen Analysen ausgeschlossen, die im vorliegenden Kapitel berichtet werden. Von den 118 verbliebenen Personen waren 80 weiblich (68\%) und 38 männlich (32\%). Ihr Durchschnittsalter betrug 22,61 Jahre (SD = 3,48), in einer Spanne zwischen 18 und 43 Jahren. Unter diesen 118 Personen befanden sich 15 Probanden, die fehlende Werte in mindestens einem der Persönlichkeitsfragebögen aufwiesen und daher weitere potenzielle Ausschlüsse darstellen. Hinsichtlich der verschiedenen Analysen dieses Kapitels ergeben sich dadurch unterschiedliche Ausschlusskriterien. Die daraus resultierenden Zusammensetzungen der Stichproben werden im Rahmen der jeweiligen Analysen berichtet.

Handlungs- und Lageorientierung, Optimismus und Pessimismus, der regulatorische Fokus sowie der Rational Thinking Style wurden als natürliche Prädiktoren auf Trait-Ebene gemessen. Die versunkenen Kosten wurden als zweistufiger Zwischensubjektfaktor (vorhanden vs. nicht vorhanden) operationalisiert. Die experimentelle Variation erfolgte jeweils in zwei separaten Sunk-Cost-Szenarien (Airplane-Szenario und DruckerpressenSzenario; beide von Arkes \& Blumer, 1985), wobei jedem Probanden gleichzeitig eine SC ${ }^{(+)}$Version des einen und eine $\mathrm{SC}^{(-)}$-Version des anderen Szenarios vorgelegt wurde. Als Kriteriumsvariablen wurden einerseits die dichotome Investitionsentscheidung und andererseits die prozentuale Investitionsbereitschaft innerhalb beider Sunk-Cost-Szenarien erhoben. 


\subsubsection{Durchführung}

Die vorliegende Untersuchung wurde als Fragebogenstudie im Labor durchgeführt. Die Akquise der Probanden erfolgte zeitgleich zu der Erhebung über Aushänge im Universitätsgebäude. Auf den Aushängen wurde mit einer Tafel Schokolade als Teilnahmevergütung geworben. Alle Interessenten mussten für die Teilnahme an der Befragung selbständig einen bestimmten Hörsaal aufsuchen. Da es sich um den größten Hörsaal der Georg-August-Universität Göttingen mit etwa 900 Sitzplätzen handelte, war es nicht notwendig, eine Obergrenze gleichzeitiger Teilnehmer festzulegen. Jeder Proband konnte direkt nach seinem Eintreffen an der fortlaufenden Erhebung teilnehmen, bis die zuvor abgezählten Fragebögen aufgebraucht waren.

An den Eingängen des Hörsaals wies je ein Plakat auf den Ablauf der Befragung hin. Diese standardisierten Informationen sollten den Teilnehmern etwaige Fragen an die Versuchsleitung ersparen, um so einen ruhigen Verlauf der stetig fortlaufenden Erhebungen zu gewährleisten. Entsprechend der Beschreibung auf dem Plakat, holten sich die Probanden beim Eintreten zuerst einen Fragebogen bei der Versuchsleitung ab. Die Reihenfolge der Fragebögen wurde zuvor von der Versuchsleitung zufällig gemischt, um potenzielle Reihenfolgeeffekte zu vermeiden. Anschließend nahmen die Probanden selbständig einen Platz im Hörsaal ein, um den Fragebogen auszufüllen. Es wurde darauf hingewiesen, dass keine Ablenkungsmittel oder Unterhaltungen gestattet seien. Nach der vollständigen Bearbeitung des Fragebogens gaben die Probanden diesen eigenständig bei der Versuchsleitung wieder ab und erhielten im Gegenzug eine Tafel Schokolade, wie zuvor als festgelegte Versuchspersonenvergütung kommuniziert. Durch die Quittierung des Erhalts auf einer Teilnehmer-Quittungsliste, wie auch durch den Einsatz eines konstanten Versuchsleiters, sollte unter anderem Mehrfachteilnahmen entgegengewirkt werden. Anschließend wurden die Probanden mit Dank von der Versuchsleitung verabschiedet, die ihnen nach der Erhebung für weiterführende Fragen über die Hintergründe der Untersuchung 
zur Verfügung stand. Die Versuchsleitung achtete darauf, dass die gesamte Phase nach der Fragebogenabgabe so leise wie möglich ablief, um die parallelen Erhebungen der anderen Probanden nicht zu stören. Eine durchschnittliche Bearbeitungsdauer der Fragebögen kann aufgrund der geschilderten Durchführungsbedingungen nicht verlässlich angegeben werden.

\subsubsection{Material}

Die ausgeteilten Fragebögen enthielten auf der ersten Seite allgemeine Instruktionen. Hierin wurde informiert, dass der Fragebogen in zwei Abschnitte zu den Themen „Persönlichkeitsmessung“ und „Entscheidungsverhalten“ aufgeteilt sei, auf die am Ende noch ein Abschlussfragebogen folge. Ferner wurde darauf hingewiesen, dass sich die Probanden bei Fragen an die Versuchsleitung wenden können und all ihre im Laufe der Studie erhobenen Daten selbstverständlich anonym seien und nicht namentlich zugeordnet würden. Darüber hinaus erhielten die Probanden noch konkrete Informationen über die Bearbeitung der Fragen im ersten Abschnitt, der zur Messung der Persönlichkeitsvariablen diente. Außerdem wurden auf der ersten Fragebogenseite das Geschlecht, das Alter, das Studienfach und die Semesteranzahl erhoben.

\subsubsection{Erster Teil: Messung der Persönlichkeitsvariablen}

Die Messung der Persönlichkeitsvariablen erfolgte auf den folgenden sechs Seiten. Hierbei wurde pro Seite je ein Persönlichkeitskonstrukt erhoben, abgesehen von der Handlungs- und Lageorientierung, deren Items sich auf drei zusammenhängende Seiten verteilten. Um mögliche Reihenfolgeeffekte zu vermeiden, wurde die Reihenfolge, in der die entsprechenden Persönlichkeitsskalen abgefragt wurden, im Rahmen der Fragebogenerstellung variiert. Die verwendeten Skalen werden im Folgenden beschrieben und finden sich zugleich in Anhang A. 


\subsection{Handlungs- und Lageorientierung}

Die Messung der Handlungs- und Lageorientierung auf Trait-Ebene (van Putten et al., 2010) erfolgte mit den gleichen 24 Items wie in der Originalstudie, allerdings in ihrer deutschen Originalfassung des „HAKEMP 90“ von Kuhl und Beckmann (1994a). Hierbei mussten sich die Probanden pro Frage für eine aus zwei vorgegebenen Antwortmöglichkeiten durch Ankreuzen entscheiden, die entweder handlungsorientiert oder lageorientiert formuliert sind (z.B. „Wenn meine Arbeit als völlig unzureichend bezeichnet wird, dann: a) lasse ich mich davon nicht lange beirren; b) bin ich zuerst wie gelähmt“").

\subsection{Optimismus und Pessimismus}

Da es sich bei der Studie von Juliusson (2006) um eine Untersuchung auf State-Ebene handelte, musste das Konstrukt des zustandsbezogenen Optimismus und Pessimismus im Rahmen dieser Untersuchung auf Trait-Ebene übertragen werden. Hierzu wurde der LifeOrientation-Test (LOT-R) von Scheier, Carver und Bridges (1994) in deutscher Übersetzung von Glaesmer, Hoyer, Klotsche und Herzberg (2008) eingesetzt, bei dem es sich den Autoren zufolge um das „mit Abstand am häufigsten eingesetzte Messinstrument zur Erfassung des dispositionellen Optimismus“ handelt (Glaesmer et al., 2008, S. 26).

Der LOT-R enthält insgesamt zehn Items. Davon beziehen sich je drei Items auf Optimismus (z.B. „Auch in ungewissen Zeiten erwarte ich normalerweise das Beste“) und drei auf Pessimismus (z.B. „Wenn bei mir etwas schief laufen kann, dann tut es das auch“); die vier übrigen sind Füllitems (z.B. „In meinem Freundeskreis fühle ich mich wohl“). Die jeweilige Zustimmungstendenz wurde auf einer fünfstufigen Antwortskala mit den Endpunkten „trifft überhaupt nicht zu“ (1) und „trifft voll und ganz zu“ (5) angegeben.

\subsection{Regulatorischer Fokus: Promotion und Prevention}

Auch der regulatorische Fokus wurde in der Originalstudie von Molden und Hui (2011) auf State-Ebene induziert und musste daher für die vorliegenden Zwecke in seiner chronischen Ausprägung auf Trait-Ebene gemessen werden. Hierfür kam die bereits erwähnte 
Skala von Lockwood et al. (2002), in der deutschsprachigen Version von Keller und Bless (2006), zum Einsatz.

Von den insgesamt 18 Items beziehen sich jeweils neun auf den Promotion Focus (z.B. „Ich stelle mir oft vor, wie ich meine Hoffnungen und Ziele erreichen werde“) und neun auf den Prevention Focus (z.B. „Im Allgemeinen konzentriere ich mich darauf, negative Ereignisse in meinem Leben zu vermeiden“). Die jeweilige Zustimmungstendenz wurde, wie bei Keller und Bless (2006), auf einer siebenstufigen Antwortskala angegeben. Die Endpunkte hießen „trifft überhaupt nicht zu“(1) und ,trifft voll und ganz zu“ (7).

\subsection{Rational Thinking Style}

Da es sich bei der Untersuchung von Wong et al. (2008) um eine Trait-Studie handelte, kamen zur Messung des Rational Thinking Styles die gleichen 20 Items der Rationalitätsskala aus Pacinis und Epsteins (1999) Rational-Experimental Inventory zum Einsatz wie in der Originalstudie, jedoch in deutscher Übersetzung.

Die Skala lässt sich seinerseits in zehn Items zur Rational Ability (RA) und zehn zum Rational Engagement (RE) unterteilen, die entweder positiv oder negativ formuliert sind (Beispiele: $\mathrm{RA}^{(+)}$: „Ich habe einen logischen Verstand“, $\mathrm{RA}^{(-)}$: „Ich bin kein besonders analytischer Denker“, $\mathrm{RE}^{(+)}$: „Ich genieße intellektuelle Herausforderungen“, $\mathrm{RE}^{(-)}$: „Die Antwort zu kennen, ohne den Grund dahinter verstehen zu müssen, ist ausreichend für mich“). Ihre Zustimmungstendenz zu den jeweiligen Aussagen gaben die Probanden auf einer fünfstufigen Antwortskala an, mit den Endpunkten „trifft überhaupt nicht zu“ (1) und „trifft voll und ganz zu“(5). 


\subsubsection{Zweiter Teil: Messung der Sunk-Cost-Neigung}

Die Erhebung der Sunk-Cost-Neigung erfolgte im Anschluss an die der Persönlichkeitsvariablen. Hierbei wurden die beiden klassischen Sunk-Cost-Szenarien von Arkes und Blumer (1985) eingesetzt, die bereits in Abschnitt 2.1.3 vorgestellt wurden; nämlich zum einen das „Airplane-Szenario“, in dem über die Forschungsfinanzierung eines Tarnflugzeugs entschieden werden soll, und zum anderen das „Druckerpressen-Szenario“, in dem über den Kauf einer gebrauchten Druckerpresse zur Modernisierung des eigenen Betriebs entschieden werden soll (siehe Anhang A). Die Reihenfolge, in der diese beiden Szenarien zu bearbeiten waren, wurde schon im Zuge der Fragebogenerstellung variiert, um auch in dieser Hinsicht Reihenfolgeeffekte zu vermeiden. Jeder Fragebogen enthielt je eine $\mathrm{SC}^{(+)}$-Version des einen Szenarios und eine $\mathrm{SC}^{(-)}$-Version des anderen Szenarios, woraus sich vier mögliche Fragebogenkombinationen ergaben.

Eingeleitet wurde die Bearbeitung der Szenarien durch den Hinweis, dass die folgenden „Szenarien zum Entscheidungsverhalten“ unabhängig voneinander sind und die Entscheidung durch Beantwortung zweier Fragen zu treffen ist. Pro Szenario wurde wie erwähnt erstens die dichotome Investitionsentscheidung als kategoriales $\mathrm{Ma}$ und zweitens die prozentuale Investitionsbereitschaft als kontinuierliches Maß erhoben. Hierdurch sollte eine bestmögliche Vergleichbarkeit der Ergebnisse dieser Untersuchung $\mathrm{zu}$ jenen der Originalstudien gewährleistet werden, in denen je eines der beiden Maße ebenfalls verwendet wurde. Die Investitionsentscheidung trafen die Probanden im Anschluss an die Frage, ob sie investieren möchten, durch ankreuzen der dichotomen Antwortmöglichkeit (ja vs. nein); ihre prozentuale Investitionsbereitschaft trugen die Probanden in einer Spanne zwischen 0\% (nicht bereit) und $100 \%$ (vollkommen bereit) in ein separates Feld ein. ${ }^{35}$

\footnotetext{
${ }^{35}$ Im Anschluss an die Bearbeitung des beschriebenen Materials folgte noch ein weiterer Fragebogen zum Zwecke einer anderen Untersuchung. Dieser war jedoch thematisch unabhängig von der vorliegenden Arbeit und wird daher nicht berichtet.
} 


\subsubsection{Dritter Teil: Abschlussfragebogen}

Nachdem alle relevanten Daten zur Persönlichkeit und zum Entscheidungsverhalten erhoben waren, folgte auf der letzten Seite ergänzend ein Abschlussfragebogen. Darin wurde zuerst gefragt, ob schon einmal an einer ähnlichen Studie teilgenommen wurde. Falls zutreffend, sollte deren Inhalt und Ablauf beschrieben werden. Als zweites sollte im Sinne einer Verdachtskontrolle niedergeschrieben werden, worum es nach Meinung des Probanden in der vorliegenden Untersuchung ging. Am Ende der letzten Seite wurde den Teilnehmern auch schriftlich gedankt und um Verschwiegenheit hinsichtlich der Inhalte dieser Untersuchung gebeten. 


\subsection{Ergebnisse}

Die dichotome Kriteriumsvariable „Investitionsentscheidung“ wurde in allen Analysen der folgenden Abschnitte mit „0“ (nicht investieren) und „1“ (investieren) codiert und in Form von logistischen Regressionsanalysen ausgewertet. Die metrische Kriteriumsvariable „Investitionsbereitschaft“ enthielt die prozentualen Angaben der Probanden mit möglichen Werten zwischen $0 \%$ und $100 \%$ und wurde stets mithilfe von linearen Regressionsanalysen ausgewertet. Die unabhängige Variable „versunkene Kosten“ wurde in beiden Szenarien jeweils mit „,0“ $\left(\mathrm{SC}^{(-)}\right)$und „1“ $\left(\mathrm{SC}^{(+)}\right)$codiert. Im Fokus aller folgenden Analysen steht die Überprüfung möglicher Interaktionseffekte zwischen den Persönlichkeitsvariablen und den versunkenen Kosten. Die Berichte erfolgen in zwei verschiedenen Abschnitten:

Im Abschnitt 5.2.1 werden separierte Analysen zu den einzelnen Persönlichkeitsvariablen berichtet, in die die jeweils anderen Variablen zunächst nicht als Prädiktoren mit einbezogen wurden. Wie erwähnt wiesen 15 der 118 verwertbaren Datensätze an unterschiedlichen Stellen des Fragebogens fehlende Werte auf. Der Vorteil dieser Einzelanalysen ist daher, dass darin alle Personen mit einbezogen werden können, die zumindest in den spezifischen Skalen der zu betrachtenden Persönlichkeitsvariablen valide Werte geliefert haben - deren fehlende Werte sich also auf die anderen, nicht betrachteten Persönlichkeitsskalen beziehen. Die daraus resultierenden, unterschiedlichen Stichproben werden vor jeder Einzelanalyse vorgestellt. Dabei wird auch kurz auf die Unterschiede eingegangen, die sich im Rahmen der integrativen Replikationsstudie gegenüber den entsprechenden Originalstudien ergeben haben.

Im Abschnitt 5.2.2 werden die integrativen Analysen berichtet, in die alle Persönlichkeitsvariablen simultan als Prädiktoren mit einbezogen wurden. Auf diese Weise lassen sich Interkorrelationen zwischen den Variablen aufdecken und feststellen, ob die Originaleffekte replizierbar sind, wenn jeweils andere Persönlichkeitsvariablen ihrerseits einen Einfluss ausüben. Aus dem hierfür verwendeten Datensatz wurden alle Personen 
ausgeschlossen, die an mindestens einer Stelle der Persönlichkeitsskalen einen fehlenden Wert aufwiesen.

\subsubsection{Einzelanalysen der Persönlichkeitsvariablen}

\subsubsection{Handlungs- und Lageorientierung}

Van Putten et al. (2010) berichteten einen signifikanten Interaktionseffekt zwischen Handlungs- und Lageorientierung und den versunkenen Kosten in der Form, dass handlungsorientierte Probanden, im Vergleich $\mathrm{zu}$ lageorientierten, ihre Investitionsentscheidung im Airplane-Szenario von Arkes und Blumer (1985) weitgehend unabhängig von den versunkenen Kosten trafen (vgl. Abschnitt 2.2.2.1).

Die Vorgehensweise der integrativen Replikationsstudie, neben der dichotomen Investitionsentscheidung auch die prozentuale Investitionsbereitschaft - sowohl im AirplaneSzenario als auch im Druckerpressen-Szenario - zu messen, stellt eine methodische Erweiterung gegenüber der Originalstudie dar. Im Gegensatz zur Originalstudie wurden die Messungen der Persönlichkeitsvariablen und der Sunk-Cost-Neigung nicht im Abstand von einer Woche durchgeführt, sondern wie beschrieben in einer Erhebungsrunde.

Wie in Abschnitt 5.1.3 erläutert, wurden die gleichen 24 Items des HAKEMP-90 zur Messung der Handlungs- und Lageorientierung eingesetzt, wie in der Originalstudie (vgl. Kuhl \& Beckmann, 1994a). Lageorientierte Antworten wurden entsprechend mit „0“ und handlungsorientierte Antworten mit „1“ codiert und anschließend summiert. Die Summenwerte, mit möglichen Ausprägungen zwischen 0 und 24, wurden auf ihren gemeinsamen Mittelwert $(M=9.60, S D=4.70)$ zentriert. Fünf der insgesamt 118 Probanden wiesen mindestens einen fehlenden Wert im HAKEMP-90 auf und wurden daher aus den folgenden Analysen ausgeschlossen. Der Stichprobenumfang umfasste somit 113 Probanden (77 weiblich [68\%] und 36 männlich [32\%]), im Gegensatz zu 75 Probanden in der Originalstudie. Das Durchschnittsalter betrug 22,50 Jahre $(S D=2,96)$, in einer Spanne 
zwischen 18 und 35 Jahren. Die Ergebnisse der durchgeführten Regressionsanalysen sind in den folgenden Tabellen aufgeführt:

\begin{tabular}{lrrrrrr} 
& B & S.E. & Wald & df & Sig. & $\operatorname{Exp}(\mathrm{B})$ \\
\hline (Constant) & -1.84 & .39 & 22.08 & 1 & .000 & .16 \\
Sunk Cost Airplane & 3.63 & .55 & 44.04 & 1 & .000 & 37.69 \\
Action Orientation $(A O)$ & .07 & .08 & .73 & 1 & .393 & 1.07 \\
AO by Sunk Cost Airplane & -.07 & .12 & .33 & 1 & .567 & .94 \\
\hline
\end{tabular}

Tabelle 14: Ergebnisse der logistischen Regressionsanalyse des Prädiktors Handlungs- und

Lageorientierung; mit der dichotomen Investitionsentscheidung als Kriteriumsvariable im Airplane-Szenario.

\begin{tabular}{lrrrrrr} 
& B & S.E. & $\beta$ & t & Sig. & r \\
\hline (Constant) & 24.45 & 3.24 & & 7.55 & .000 & \\
Sunk Cost Airplane & 45.33 & 4.60 & .69 & 9.85 & .000 & .69 \\
Action Orientation $(A O)$ & .43 & .68 & .06 & .63 & .530 & .04 \\
AO by Sunk Cost Airplane & -.75 & .98 & -.07 & -.76 & .449 & -.01 \\
\hline
\end{tabular}

Tabelle 15: Ergebnisse der linearen Regressionsanalyse des Prädiktors Handlungs- und Lageorientierung; mit der prozentualen Investitionsbereitschaft als Kriteriumsvariable im Airplane-Szenario.

\begin{tabular}{lrrrrrr} 
& B & S.E. & Wald & df & Sig. & $\operatorname{Exp}(\mathrm{B})$ \\
\hline (Constant) & 1.54 & .36 & 18.84 & 1 & .000 & 4.68 \\
Sunk Cost Printer & -.70 & .47 & 2.28 & 1 & .131 & .50 \\
Action Orientation $(A O)$ & .07 & .08 & .69 & 1 & .405 & 1.07 \\
AO by Sunk Cost Printer & .04 & .10 & .14 & 1 & .714 & 1.04 \\
\hline
\end{tabular}

Tabelle 16: Ergebnisse der logistischen Regressionsanalyse des Prädiktors Handlungs- und

Lageorientierung; mit der dichotomen Investitionsentscheidung als Kriteriumsvariable im DruckerpressenSzenario.

\begin{tabular}{lrrrrrr} 
& B & S.E. & $\beta$ & t & Sig. & $\mathrm{r}$ \\
\hline (Constant) & 70.68 & 3.50 & & 20.18 & .000 & \\
Sunk Cost Printer & -12.62 & 4.93 & -.23 & -2.56 & .012 & -.24 \\
Action Orientation $(A O)$ & .15 & .76 & .03 & .202 & .840 & .16 \\
AO by Sunk Cost Printer & 1.30 & 1.05 & .16 & 1.24 & .219 & .19 \\
\hline
\end{tabular}

Tabelle 17: Ergebnisse der linearen Regressionsanalyse des Prädiktors Handlungs- und Lageorientierung, mit der prozentualen Investitionsbereitschaft als Kriteriumsvariable im Druckerpressen-Szenario.

Im Einklang mit der Originalstudie fand sich im Airplane-Szenario für beide Kriteriumsvariablen ein signifikanter Sunk-Cost-Haupteffekt, wonach die Probanden auf Stufe vorhandener versunkener Kosten einerseits häufiger investierten $\left(M_{(S C+)}=85,7 \%\right.$ vs. $\left.M_{(S C-)}=14,0 \%\right)$ und andererseits auch eine höhere prozentuale Investitionsbereitschaft aufwiesen $\left(M_{(S C+)}=69,7 \%\right.$ vs. $\left.M_{(S C-)}=24,4 \%\right)$ als Probanden auf der Stufe ohne versunkene Kosten. Damit konnte der grundlegende Befund von Arkes und Blumer (1985) repliziert werden. Im Druckerpressen-Szenario konnte nur in der Analyse der prozentualen 
Investitionsbereitschaft ein signifikanter Sunk-Cost-Effekt nachgewiesen werden $\left(M_{(S C+)}=\right.$ $57,8 \%$ vs. $\left.M_{(S C-)}: M=70,7 \%\right)$. In der Analyse der dichotomen Investitionsentscheidung wurde der Effekt nicht signifikant $\left(M_{(S C+)}=68,4 \%\right.$ vs. $\left.M_{\left(S C_{-}\right)}=82,1 \%\right)$. In beiden Fällen wiesen die Probanden aber bei vorhandenen versunkenen Kosten eine geringere Investitionsneigung auf als in Abwesenheit versunkener Kosten, was inhaltlich der Richtung des grundlegenden Effekts von Arkes und Blumer (1985) entspricht.

Die zentrale Interaktion zwischen Handlungs- und Lageorientierung und den versunkenen Kosten konnte hingegen für keine der beiden Kriteriumsvariablen nachgewiesen werden, weder im Airplane-Szenario noch im Druckerpressen-Szenario. Die in der Originalstudie berichtete moderierende Wirkung von Handlungs- und Lageorientierung, in der sich handlungsorientierte Personen im Gegensatz zu lageorientierten Personen relativ robust gegenüber dem Sunk-Cost-Effekt zeigten, erscheint daher auch auf Grundlage der vorliegenden Ergebnisse fragwürdig. ${ }^{36}$

\subsubsection{Optimismus und Pessimismus}

Juliusson (2006) berichtete einen moderierenden Einfluss von zustandsbezogenem Optimismus und Pessimismus auf den Sunk-Cost-Effekt, indem hohe versunkene Kosten das generell erhöhte Investitionsverhalten der Optimisten sowie das generell erhöhte Abbruchverhalten der Pessimisten jeweils verstärkten, verglichen mit niedrigen versunkenen Kosten (vgl. Abschnitt 2.2.2.2).

In der Originalstudie wurden einerseits die dichotome Investitionsentscheidung und andererseits die Überzeugung der Probanden von dieser Entscheidung erhoben. Der zentrale Interaktionseffekt fand sich allerdings nur in der Analyse der dichotomen

\footnotetext{
${ }^{36}$ Es wurden zwei zusätzliche Kontrollanalysen durchgeführt: In der ersten Analyse wurden Alter, Geschlecht, Studiengang und Semesteranzahl als Kontrollvariablen berücksichtigt. In der zweiten wurden keine Ausschlüsse von Personen vorgenommen, die in den Persönlichkeitsfragebögen fehlende Werte aufwiesen $(N=118)$. Anstelle der fehlenden Werte wurden die jeweiligen Mittelwerte der entsprechenden Items für Handlungs- und Lageorientierung eingesetzt, um die Summenvariable wie beschrieben berechnen zu können. In beiden Kontrollanalysen änderte sich jedoch nichts an der zentralen Befundlage.
} 
Investitionsentscheidung, weswegen im Rahmen der integrativen Replikationsstudie auf eine zusätzliche Erhebung der Überzeugung verzichtet wurde. Als zweite Kriteriumsvariable diente, wie bereits erläutert, die prozentuale Investitionsbereitschaft. Die von Juliusson (2006) eigens kreierten Sunk-Cost-Szenarien wurden im Rahmen der integrativen Replikationsstudie durch die zwei vorgestellten, empirisch gut bewährten Szenarien von Arkes und Blumer (1985) ersetzt. Schließlich wurden Optimismus und Pessimismus nicht wie in der Originalstudie experimentell induziert, sondern ihre chronischen Ausprägungen auf TraitEbene erhoben. Die Akquise der Probanden erfolgte über Aushänge im Universitätsgebäude und nicht wie bei Juliusson (2006) im Anschluss an eine Vorlesung.

Die Messung des chronischen Optimismus und Pessimismus wurde wie beschrieben mithilfe der ins Deutsche übersetzten LOT-R-Skala von Scheier et al. (1994) realisiert. Entsprechend der von den Autoren aufgestellten Konvention, wurde die Skala als eindimensionales Messinstrument ausgewertet. Diese Form der Auswertung kommt der aus der Originalstudie, in der Optimismus und Pessimismus als zweistufiger Zwischensubjektfaktor operationalisiert wurden, am nächsten. Hierfür wurden zunächst die Werte der drei Pessimismus-Items invertiert und anschließend zusammen mit den Werten der drei Optimismus-Items summiert, ohne Einbezug der vier Füllitems. Daraus ergab sich ein übergreifender Optimismus-Summenscore mit möglichen Werten zwischen 6 und 30, die anschließend auf ihren gemeinsamen Mittelwert $(M=21.36, S D=3.87)$ zentriert wurden. Analog zur Analyse der Handlungs- und Lageorientierung spiegeln positive Werte so eine stärkere Optimismusneigung und negative Werte eine stärkere Pessimismusneigung wider.

Vier der 118 Probanden wiesen mindestens einen fehlenden Wert im LOT-R auf und wurden daher aus den folgenden Analysen ausgeschlossen. Der Stichprobenumfang umfasste somit 114 Probanden (78 weiblich [68\%] und 36 männlich [32\%]), im Gegensatz zu 52 Probanden in der Originalstudie. Das Durchschnittsalter betrug 22,61 Jahre $(S D=3,52)$, in 
einer Spanne zwischen 18 und 43 Jahren. Die Ergebnisse der durchgeführten

Regressionsanalysen sind in den folgenden Tabellen aufgeführt:

\begin{tabular}{lrrrrrr} 
& B & S.E. & Wald & df & Sig. & $\operatorname{Exp}(\mathrm{B})$ \\
\hline (Constant) & -2.07 & .46 & 20.27 & 1 & .000 & .13 \\
Sunk Cost Airplane & 4.02 & .61 & 42.96 & 1 & .000 & 55.90 \\
Optimism vs. Pessimism (OP) & .19 & .11 & 3.21 & 1 & .073 & 1.21 \\
OP by Sunk Cost Airplane & -.18 & .15 & 1.34 & 1 & .246 & .84 \\
\hline
\end{tabular}

Tabelle 18: Ergebnisse der logistischen Regressionsanalyse des Prädiktors Optimismus und Pessimismus; mit der dichotomen Investitionsentscheidung als Kriteriumsvariable im Airplane-Szenario.

\begin{tabular}{lrrrrrr} 
& B & S.E. & $\beta$ & t & Sig. & r \\
\hline (Constant) & 23.14 & 3.10 & & 7.47 & .000 & \\
Sunk Cost Airplane & 47.61 & 4.42 & .72 & 10.78 & .000 & .72 \\
Optimism vs. Pessimism (OP) & .71 & .77 & .08 & .92 & .358 & .00 \\
OP by Sunk Cost Airplane & -.92 & 1.16 & -.07 & -.79 & .429 & -.04 \\
\hline
\end{tabular}

Tabelle 19: Ergebnisse der linearen Regressionsanalyse des Prädiktors Optimismus und Pessimismus; mit der prozentualen Investitionsbereitschaft als Kriteriumsvariable im Airplane-Szenario.

\begin{tabular}{lrrrrrr} 
& B & S.E. & Wald & df & Sig. & Exp(B) \\
\hline (Constant) & 1.52 & .35 & 19.05 & 1 & .000 & 4.59 \\
Sunk Cost Printer & -.86 & .45 & 3.63 & 1 & .057 & .42 \\
Optimism vs. Pessimism $(O P)$ & -.03 & .10 & .09 & 1 & .759 & .97 \\
OP by Sunk Cost Printer & .17 & .12 & 1.84 & 1 & .175 & 1.18 \\
\hline
\end{tabular}

Tabelle 20: Ergebnisse der logistischen Regressionsanalyse des Prädiktors Optimismus und Pessimismus; mit der dichotomen Investitionsentscheidung als Kriteriumsvariable im Druckerpressen-Szenario.

\begin{tabular}{lrrrrrr} 
& B & S.E. & $\beta$ & $\mathrm{t}$ & Sig. & $\mathrm{r}$ \\
\hline (Constant) & 70.54 & 3.60 & & 19.61 & .000 & \\
Sunk Cost Printer & -14.48 & 5.04 & -.26 & -2.87 & .005 & -.26 \\
Optimism vs. Pessimism (OP) & -.97 & .99 & -.14 & -.99 & .326 & .02 \\
OP by Sunk Cost Printer & 2.14 & 1.32 & .22 & 1.63 & .107 & .11 \\
\hline
\end{tabular}

Tabelle 21: Ergebnisse der linearen Regressionsanalyse des Prädiktors Optimismus und Pessimismus; mit der prozentualen Investitionsbereitschaft als Kriteriumsvariable im Druckerpressen-Szenario.

Das Befundmuster hinsichtlich der Sunk-Cost-Haupteffekte entsprach dem aus der ersten Einzelanalyse der Handlungs- und Lageorientierung. Der Nachweis eines Interaktionseffekts zwischen Optimismus und Pessimismus und den versunkenen Kosten schlug allerdings in allen durchgeführten Analysen fehl. Von einer moderierenden Wirkung von TraitOptimismus und Pessimismus auf den Sunk-Cost-Effekt kann daher auf Basis der vorliegenden Ergebnisse nicht ausgegangen werden. Abseits der zentralen Fragestellung 
konnte auch der Haupteffekt aus der Originalstudie, in der Optimisten eine signifikant höhere Investitionsneigung als Pessimisten zeigten, in dieser Studie nicht repliziert werden. ${ }^{37}$

\subsubsection{Regulatorischer Fokus: Promotion und Prevention}

Molden und Hui (2011) berichteten, dass in ihrer Studie Probanden mit einem Promotion Focus eine signifikant geringere Investitionsneigung innerhalb des Airplane-Szenarios (Arkes \& Blumer, 1985) aufwiesen als Probanden mit einem Prevention Focus oder Probanden in der Kontrollgruppe. Daraus leiteten die Autoren ab, dass ein Promotion Focus hilfreich sei, um versunkene Kosten besser ignorieren zu können (vgl. Abschnitt 2.2.2.3). Diese Aussage kann jedoch strenggenommen durch das originale Versuchsdesign nicht gestützt werden, da hierin alleine die $\mathrm{SC}^{(+)}$-Version des Airplane-Szenarios eingesetzt wurde (vgl. Abschnitt 2.1.3).

Über das Design der Originalstudie hinausgehend wurde daher in der integrativen Replikationsstudie ein erweitertes Design eingesetzt, in das eine zusätzliche $\mathrm{SC}^{(-)}$-Faktorstufe integriert wurde, um eine mögliche Interaktion zwischen den versunkenen Kosten und dem regulatorischen Fokus aufdecken zu können. Der Hintergrund wurde bereits im Rahmen der Berichte der direkten Replikationsstudien, in denen ebenso verfahren wurde, erläutert (vgl. Abschnitt 4.3). Der gleichzeitige Einsatz der beiden beschriebenen Sunk-Cost-Szenarien sowie die Erhebung zweier Kriteriumsvariablen, stellen eine zusätzliche methodische Erweiterung gegenüber der Originalstudie dar. Allerdings wurden der Promotion Focus und der Prevention Focus nicht wie in der Originalstudie experimentell durch eine Schreibaufgabe induziert, sondern ihre chronischen Ausprägungen auf Trait-Ebene erhoben. Auf die Bildung einer Kontrollgruppe, in der die Probanden einen Aufsatz über ihre typischen täglichen

\footnotetext{
${ }^{37}$ Es wurden drei Kontrollanalysen durchgeführt: In der ersten wurden Alter, Geschlecht, Studiengang und Semesteranzahl als Kontrollvariablen berücksichtigt. In der zweiten wurden keine Ausschlüsse von Personen vorgenommen, die in den Persönlichkeitsfragebögen fehlende Werte aufwiesen $(N=118)$. Anstelle der fehlenden Werte wurden hier die jeweiligen Mittelwerte der entsprechenden Items für Optimismus und Pessimismus eingesetzt, um die Summenvariable wie beschrieben berechnen zu können. In der dritten Kontrollanalyse erfolgte ein Ausschluss aller wirtschaftswissenschaftlichen Studierenden, da diese in der Originalstudie nicht miterhoben wurden. Keine der drei angestellten Kontrollanalysen erbrachte Änderungen an der zentralen Befundlage.
} 
Aktivitäten verfassen sollten, wurde daher verzichtet. Während die Befragung bei Molden und Hui (2011) online realisiert wurde, fand die Erhebung im Rahmen der Replikationsstudie papierbasiert in einem Laborraum statt.

Die zur Messung des chronischen regulatorischen Fokus eingesetzte Lockwood-Skala wurde wie bei Keller und Bless (2006) als eindimensionales Messinstrument ausgewertet, was der Auswertung in der Originalstudie, in der Promotion und Prevention jeweils als zwei Stufen eines Zwischensubjektfaktors operationalisiert wurden, am nächsten kommt. Analog zu Keller und Bless (2006) wurden zunächst ein Promotion-Summenscore $(M=45.50 ; S D=$ 8.34) und ein Prevention-Summenscore $(M=37.14 ; S D=9.60)$, mit möglichen Werten zwischen 9 und 63, aus den jeweiligen Items gebildet. Das relative, eindimensionale Maß für den regulatorischen Fokus ergab sich durch Subtraktion des z-standardisierten PreventionScores von dem z-standardisierten Promotion-Score. Die Werte des regulatorischen Fokus, die in einer Spanne zwischen -2,19 und 3,40 um den Mittelwert 0 streuten $(S D=1.05)$, repräsentierten bei einem positiven Vorzeichen eine relative Promotion-Neigung und bei einem negativen Vorzeichen eine relative Prevention-Neigung der Probanden.

Fünf der 118 Probanden wiesen mindestens einen fehlenden Wert in der Lockwood-Skala auf und wurden aus den folgenden Analysen ausgeschlossen. Der Stichprobenumfang umfasste daher 113 Probanden (77 weiblich [68\%] und 36 männlich [32\%]), im Gegensatz zu 114 Probanden in der Originalstudie, in der allerdings die versunkenen Kosten wie erwähnt nicht variiert wurden. Das Durchschnittsalter betrug 22,40 Jahre $(S D=2,96)$, in einer Spanne zwischen 18 und 35 Jahren. ${ }^{38}$ Die Ergebnisse der durchgeführten Regressionsanalysen sind in den folgenden Tabellen aufgeführt:

\footnotetext{
${ }^{38}$ Die Nationalität und der höchste Bildungsabschluss wurden im Rahmen dieser Untersuchung nicht explizit abgefragt. Auf Basis der berichteten Variablen „Studienfach“ und „Semesteranzahl“ wird allerdings klar, dass es sich um eine studentische Stichprobe im durchschnittlich vierten Semester $(S D=2,76)$ handelte. Daraus ließe sich ableiten, dass die Teilnehmer überwiegend die Hochschulreife als höchsten Bildungsabschluss besaßen. Im Rahmen der Erhebung entstand zudem der Eindruck, dass es sich dem Augenschein nach - zu einem vergleichbar hohen Anteil wie in den Replikationsstudien - um deutsche Teilnehmer gehandelt haben dürfte.
} 


\begin{tabular}{lrrrrrr} 
& B & S.E. & Wald & df & Sig. & $\operatorname{Exp}(\mathrm{B})$ \\
\hline (Constant) & -2.05 & .46 & 20.20 & 1 & .000 & .13 \\
Sunk Cost Airplane & 3.87 & .60 & 42.17 & 1 & .000 & 47.86 \\
Regulatory Focus $(R F)$ & .79 & .35 & 5.17 & 1 & .023 & 2.19 \\
RF by Sunk Cost Airplane & -.71 & .52 & 1.85 & 1 & .174 & .49 \\
\hline
\end{tabular}

Tabelle 22: Ergebnisse der logistischen Regressionsanalyse des Prädiktors regulatorischer Fokus; mit der dichotomen Investitionsentscheidung als Kriteriumsvariable im Airplane-Szenario.

\begin{tabular}{lrrrrrr} 
& B & S.E. & $\beta$ & t & Sig. & r \\
\hline (Constant) & 24.02 & 3.24 & & 7.43 & .000 & \\
Sunk Cost Airplane & 45.52 & 4.55 & .69 & 10.00 & .000 & .69 \\
Regulatory Focus $(R F)$ & 4.62 & 2.98 & .15 & 1.55 & .123 & .08 \\
RF by Sunk Cost Airplane & -4.01 & 4.38 & -.09 & -.914 & .362 & .01 \\
\hline
\end{tabular}

Tabelle 23: Ergebnisse der linearen Regressionsanalyse des Prädiktors regulatorischer Fokus; mit der prozentualen Investitionsbereitschaft als Kriteriumsvariable im Airplane-Szenario.

\begin{tabular}{lrrrrrr} 
& B & S.E. & Wald & df & Sig. & Exp(B) \\
\hline (Constant) & 1.44 & .34 & 18.14 & 1 & .000 & 4.21 \\
Sunk Cost Printer & -.51 & .48 & 1.14 & 1 & .286 & .60 \\
Regulatory Focus $(R F)$ & .14 & .34 & .16 & 1 & .694 & 1.15 \\
RF by Sunk Cost Printer & .86 & .53 & 2.60 & 1 & .107 & 2.36 \\
\hline
\end{tabular}

Tabelle 24: Ergebnisse der logistischen Regressionsanalyse des Prädiktors regulatorischer Fokus; mit der dichotomen Investitionsentscheidung als Kriteriumsvariable im Druckerpressen-Szenario.

\begin{tabular}{lrrrrrr} 
& B & S.E. & $\beta$ & $\mathrm{t}$ & Sig. & $\mathrm{r}$ \\
\hline (Constant) & 70.97 & 3.50 & & 20.29 & .000 & \\
Sunk Cost Printer & -13.53 & 4.97 & -.25 & -2.72 & .008 & -.25 \\
Regulatory Focus $($ RF $)$ & -.00 & 3.51 & .00 & .00 & 1.000 & .15 \\
RF by Sunk Cost Printer & 7.32 & 4.78 & .21 & 1.53 & .128 & .20 \\
\hline
\end{tabular}

Tabelle 25: Ergebnisse der linearen Regressionsanalyse des Prädiktors regulatorischer Fokus; mit der prozentualen Investitionsbereitschaft als Kriteriumsvariable im Druckerpressen-Szenario.

Das Befundmuster der Sunk-Cost-Haupteffekte entsprach dem aus den vorangegangenen Analysen zu Handlungs- und Lageorientierung sowie Optimismus und Pessimismus. Der zentrale Nachweis einer Interaktion zwischen dem regulatorischen Fokus und den versunkenen Kosten schlug allerdings in den vier durchgeführten Analysen fehl. Die Aussage von Molden und Hui (2011), wonach ein Promotion Focus hilfreich sei, um versunkene Kosten besser ignorieren zu können, konnte also nicht bestätigt werden.

Abseits der zentralen Fragestellung fand sich ein signifikanter Haupteffekt für den regulatorischen Fokus, wonach Probanden mit steigenden Promotionwerten zunehmend im Airplane-Szenario investierten. Dieser Befund steht allerdings im direkten Gegensatz zur 
Originalstudie, in der die Probanden mit einem Promotion Focus eigentlich die niedrigste Investitionsneigung aller Vergleichsbedingungen aufwiesen. ${ }^{39}$

\subsubsection{Rational Thinking Style}

Wong et al. (2008) berichteten, dass Personen mit hohen Ausprägungen des Rational Thinking Styles eine höhere Investitionsbereitschaft in dem von ihnen modifizierten AirplaneSzenario zeigten als Personen mit niedrigen Ausprägungen (vgl. Abschnitt 2.2.2.4). Allerdings wurde auch im Rahmen dieser Studie nur eine $\mathrm{SC}^{(+)}$-Version des Szenarios eingesetzt.

Um zu testen, ob es sich bei dem Rational Thinking Style um einen spezifischen Moderator für den Sunk-Cost-Effekt handelt, wurde im Rahmen der integrativen Replikationsstudie eine kontrastierende $\mathrm{SC}^{(-)}$-Faktorstufe in das Versuchsdesign mit aufgenommen, wie zuvor erläutert. Die Verwendung des originalen Airplane-Szenarios stellte eine weitere Veränderung gegenüber der Originalstudie dar, ebenso wie der zusätzliche Einsatz des Druckerpressen-Szenarios und die ergänzende Erhebung der dichotomen Investitionsentscheidung. Im Gegensatz zur Originalstudie wurde zuerst der Rational Thinking Style und erst danach die Sunk-Cost-Neigung gemessen. Um die Vergleichbarkeit zur Originalstudie nicht infrage zu stellen, wurde, wie in Abschnitt 4.4.1.2 dargelegt, eine separate Vorstudie im Rahmen dieser Arbeit durchgeführt, in der sich keine Einflüsse der Erhebungsreihenfolge feststellen ließen (siehe Anhang B). Eine Operationalisierung von Framing, als zusätzlicher Zwischensubjektfaktor, fand im Rahmen der integrativen Replikationsstudie nicht statt, da das originale Airplane-Szenario von Arkes und Blumer

\footnotetext{
39 In einer an die Originalstudie angelehnten Analyse der $\mathrm{SC}^{(+)}$-Bedingungen fanden sich für beide Kriteriumsvariablen im Druckerpressen-Szenario signifikante Haupteffekte für den regulatorischen Fokus, wonach sich Probanden bei steigenden Promotionwerten zunehmend investitionsfreudiger zeigten (dichotome Investitionsentscheidung: $\quad B=.99, S E=.41 ; \quad \chi^{2}[1, N=56]=5.94, \quad p=.015, \quad$ OR $=2.70 ;$ prozentuale Investitionsbereitschaft: $\beta=.29, t[54]=2.20, p=.032, r=.29)$. Zwei weitere Kontrollanalysen, eine unter Einbezug von Alter, Geschlecht, Studiengang und Semesteranzahl als Kontrollvariablen und eine unter Berücksichtigung aller 118 Probanden (mit den Item-Mittelwerten anstelle der fehlenden Werte in der Lockwood-Skala), änderten nichts an der zentralen Befundlage.
} 
(1985) keine derartige Manipulation vorsieht und dieser Faktor schon in der Originalstudie keine Relevanz besaß (Wong et al., 2008, S. 253). Auf eine Abfrage des Verantwortlichkeitsempfindens hinsichtlich der initiale Investition und des gestarteten Projekts wurde ebenfalls verzichtet, da es innerhalb der neu eingeführten $\mathrm{SC}^{(-)}$-Bedingung weder eine initiale Investition noch ein bereits gestartetes Projekt gab.

Wie beschrieben, wurden zur Messung des Rational Thinking Styles die gleichen 20 Items der Rationalitätsskala aus Pacinis und Epsteins (1999) Rational-Experimental Inventory verwendet wie in der Originalstudie. Im Zuge der Auswertung wurden zunächst alle negativ formulierten Items invertiert (vgl. Pacini \& Epstein, 1999, S. 976) und hieraus die Subskalen Rational Ability (RA, $M=3,49, S D=.65$ ) und Rational Engagement (RE, $M=3,58, S D=$ .64) gebildet. Parallel erfolgte auch die Bildung der Rational Thinking Style Gesamtskala (RTS, $M=3,53, S D=.59$ ) aus dem Mittelwert aller Items. Sechs der 118 Probanden wiesen mindestens einen fehlenden Wert im Rationalitätsfragebogen auf und wurden daher aus den folgenden Analysen ausgeschlossen. Der Stichprobenumfang umfasste somit 112 Probanden (75 weiblich [67\%] und 37 männlich [33\%]), im Gegensatz zu 166 Probanden in der Originalstudie, in der die versunkenen Kosten wie bereits erwähnt nicht variiert wurden. Das Durchschnittsalter betrug 22,55 Jahre $(S D=3,51)$, in einer Spanne zwischen 18 und 43 Jahren.

Die in der Originalstudie berichteten Korrelationen der drei Rationalitätsskalen untereinander konnten in der vorliegenden Untersuchung bestätigt werden (RA \& RTS: $r=$ .91 , RE \& RTS: $r=.91$, RA \& RE: $r=.65 ; p s<.001)$. Es fanden sich ebenfalls signifikante Korrelationen zwischen den versunkenen Kosten und beiden Kriteriumsvariablen im Airplane-Szenario $(r s>.71 ; p s<.001)$. Im Druckerpressen-Szenario zeigte sich nur die Korrelation zwischen der Investitionsbereitschaft und den versunkenen Kosten signifikant ( $r$ $=-.25 ; p<.001)$. Die Ergebnisse der durchgeführten Regressionsanalysen sind in den nun folgenden Tabellen aufgeführt: 


\begin{tabular}{lrrrrrr} 
& B & S.E. & Wald & df & Sig. & $\operatorname{Exp}(\mathrm{B})$ \\
\hline (Constant) & -3.19 & 2.25 & 2.00 & 1 & .157 & .04 \\
Sunk Cost Airplane & 6.82 & 3.70 & 3.40 & 1 & .065 & 916.45 \\
Rational Thinking Style (RTS) & .40 & .62 & .41 & 1 & .523 & 1.49 \\
RTS by Sunk Cost Airplane & -.86 & 1.00 & .74 & 1 & .391 & .42 \\
\hline
\end{tabular}

Tabelle 26: Ergebnisse der logistischen Regressionsanalyse des Prädiktors Rational Thinking Style; mit der dichotomen Investitionsentscheidung als Kriteriumsvariable im Airplane-Szenario.

\begin{tabular}{lrrrrrr} 
& B & S.E. & $\beta$ & t & Sig. & r \\
\hline (Constant) & 26.60 & 17.70 & & 1.50 & .136 & \\
Sunk Cost Airplane & 61.71 & 28.37 & .92 & 2.18 & .032 & .71 \\
Rational Thinking Style (RTS) & -.88 & 5.02 & -.02 & -.18 & .861 & .04 \\
RTS by Sunk Cost Airplane & -3.90 & 7.90 & -.21 & -.49 & .623 & .69 \\
\hline
\end{tabular}

Tabelle 27: Ergebnisse der linearen Regressionsanalyse des Prädiktors Rational Thinking Style; mit der prozentualen Investitionsbereitschaft als Kriteriumsvariable im Airplane-Szenario.

\begin{tabular}{lrrrrrr} 
& B & S.E. & Wald & df & Sig. & $\operatorname{Exp}(\mathrm{B})$ \\
\hline (Constant) & .40 & 2.32 & .03 & 1 & .864 & 1.49 \\
Sunk Cost Printer & -.98 & 2.80 & .12 & 1 & .726 & .37 \\
Rational Thinking Style (RTS) & .28 & .65 & .19 & 1 & .662 & 1.33 \\
RTS by Sunk Cost Printer & .08 & .79 & .01 & 1 & .919 & 1.08 \\
\hline
\end{tabular}

Tabelle 28: Ergebnisse der logistischen Regressionsanalyse des Prädiktors Rational Thinking Style; mit der dichotomen Investitionsentscheidung als Kriteriumsvariable im Druckerpressen-Szenario.

\begin{tabular}{lrrrrrr} 
& B & S.E. & $\beta$ & $\mathrm{t}$ & Sig. & $\mathrm{r}$ \\
\hline (Constant) & 73.73 & 25.15 & & 2.93 & .004 & \\
Sunk Cost Printer & -24.83 & 32.18 & -.45 & -.77 & .442 & -.25 \\
Rational Thinking Style (RTS) & -.84 & 6.92 & -.02 & -.12 & .904 & .05 \\
RTS by Sunk Cost Printer & 3.20 & 8.96 & .21 & .36 & .721 & -.23 \\
\hline
\end{tabular}

Tabelle 29: Ergebnisse der linearen Regressionsanalyse des Prädiktors Rational Thinking Style; mit der prozentualen Investitionsbereitschaft als Kriteriumsvariable im Druckerpressen-Szenario.

Insgesamt konnte nur in der Analyse der prozentualen Investitionsbereitschaft im Airplane-Szenario ein signifikanter Sunk-Cost-Effekt nachgewiesen werden. Interaktionen zwischen dem Rational Thinking Style und den versunkenen Kosten blieben in den vier durchgeführten Analysen vollständig aus. Auf Basis dieser Befunde gibt es somit keine Hinweise darauf, dass von dem Rational Thinking Style eine moderierende Wirkung auf den Sunk-Cost-Effekt ausgehen könnte.

Abseits der zentralen Fragestellung konnte der von Wong et al. (2008) berichtete Haupteffekt des RTS auf die Investitionsbereitschaft nicht repliziert werden. Wie in der Originalstudie wurden auch in der integrativen Replikationsstudie alle bisherigen Analysen 
ebenfalls mit den Subskalen RA und RE, anstelle der RTS Gesamtskala, durchgeführt. Auch hierbei zeigten sich durchweg keine signifikanten Haupteffekte für die versunkenen Kosten, die Subskalen RA und RE oder Interaktionseffekte zwischen ihnen. ${ }^{40}$

\subsubsection{Integrative Analysen der Persönlichkeitsvariablen}

In diesem Abschnitt werden die integrativen Analysen der vorliegenden Untersuchung berichtet, in die alle Persönlichkeitsvariablen simultan als Prädiktoren mit aufgenommen wurden. Von den 118 Versuchspersonen wurden jene 15 ausgeschlossen, die mindestens einen fehlenden Wert in den Persönlichkeitsfragebögen aufwiesen (vgl. Abschnitt 5.1.1). Der Stichprobenumfang umfasste daher 103 Versuchspersonen (70 weiblich [68\%] und 33 männlich [32\%]). Ihr Durchschnittsalter betrug 22,35 Jahre $(S D=2,98)$, in einer Spanne zwischen 18 und 35 Jahren.

Im Folgenden werden zunächst die Analysen für beide Kriteriumsvariablen (dichotome Investitionsentscheidung vs. prozentuale Investitionsbereitschaft) im Airplane-Szenario vorgestellt und im Anschluss folgen die entsprechenden Analysen des DruckerpressenSzenarios. Da die verwendeten Persönlichkeitsskalen dieselben sind, deren Berechnungen bereits im Verlauf dieses Kapitels beschrieben wurden, wird diesbezüglich auf eine erneute Erläuterung verzichtet. Auch die Variablencodierungen wurden unverändert so

\footnotetext{
${ }^{40}$ In einer an die Originalstudie angelehnten Analyse der $\mathrm{SC}^{(+)}$-Bedingungen fand sich nur für RA ein signifikanter Haupteffekt $(\beta=-.40, p=.021)$, wonach Probanden mit steigenden RA-Ausprägungen im Gegensatz zur Originalstudie eine abnehmende Investitionsbereitschaft im Airplane-Szenario aufwiesen.

In Analysen unter Einschluss von Alter und Geschlecht als Kontrollvariablen, wie es auch in der Originalstudie vollzogen wurde, zeigten sich in keiner Berechnung signifikante Haupt- oder Interaktionseffekte. Bei einer zusätzlichen Hinzunahme des Studiengangs und der Semesterzahl, als weitere Kontrollvariablen, zeigten sich: a) ein SC-Effekt für die Investitionsbereitschaft (Airplane-Szenario, Analyse RA/RE $[B=57.78, p=.047]$ ); b) signifikante SC-Effekte für die Investitionsentscheidung (Airplane-Szenario, Analysen RTS sowie RA/RE [alle $B$ s positiv, $p \mathrm{~s}<.034]$ ); c) ein signifikanter Haupteffekt für RE (Investitionsbereitschaft, Druckerpresse $(B=-$ $18.30, p=.027)$.

In Kontrollanalysen unter Berücksichtigung aller 118 Probanden, fanden sich a) signifikante SC-Effekte für beide Kriteriumsvariablen (Airplane-Szenario, Analysen RTS sowie RA/RE [alle Bs positiv, $p$ s < .047]); b) ein signifikanter Haupteffekt für RE (Investitionsbereitschaft, Druckerpressen-Szenario $[\beta=-.45, p=.020]$ ).

Auch nach den Kontrollanalysen bleibt insgesamt festzuhalten, dass in keiner aller durchgeführten Berechnungen die Befunde von Wong et al. (2008) repliziert werden konnten und keinerlei Interaktionen nachweisbar waren.
} 
vorgenommen, wie bereits einleitend in Abschnitt 5.2 beschrieben. Im zentralen Interesse aller nun folgenden Analysen steht wieder der Nachweis von Interaktionen zwischen den Persönlichkeitsvariablen und den versunkenen Kosten.

\subsubsection{Analysen des Airplane-Szenarios}

Tabelle 30 liefert zunächst einen Überblick über die Mittelwerte, Standardabweichungen und Korrelationskoeffizienten aller Variablen aus den Analysen des Airplane-Szenarios in der integrativen Replikationsstudie. Erwartungsgemäß zeigten sich jeweils signifikante und positive Korrelationen der versunkenen Kosten mit beiden Kriteriumsvariablen (dichotome Investitionsentscheidung und prozentuale Investitionsbereitschaft). Die zwei Kriteriumsvariablen korrelierten ihrerseits ebenfalls positiv und signifikant miteinander. Außerdem fanden sich signifikante Interkorrelationen in mittlerer Höhe zwischen den vier Persönlichkeitsvariablen. Eine schwache positive Korrelation zwischen dem regulatorischen Fokus und der prozentualen Investitionsbereitschaft zeigte sich nur einseitig signifikant.

\begin{tabular}{llllllllll} 
& & $\mathrm{M}$ & $\mathrm{SD}$ & 1 & 2 & 3 & 4 & 5 & 6 \\
\hline 1. & Investition Airplane & .50 & .50 & --- & & & & \\
2. & Willingness Airplane & 47.02 & 32.91 & $.83^{* * *}$ & --- & & & \\
3. & Sunk Cost Airplane & .49 & .50 & $.73^{* * *}$ & $.72^{* * *}$ & --- & & \\
4. & Action Orientation & .00 & 4.52 & .14 & .11 & .10 & --- & & \\
5. & Optimism vs. Pessimism & .00 & 3.84 & .11 & .05 & -.01 & $.50^{* * *}$ & --- \\
\hline 6. & Regulatory Focus & .00 & 1.03 & .13 & $.10^{\dagger}$ & -.01 & $.46^{* * *}$ & $.51^{* * *}$ & --- \\
7. & Rational Thinking Style & 3.49 & .59 & .13 & .07 & .14 & $.40^{* * *}$ & $.31^{* *}$ & $.48^{* * *}$ \\
\hline
\end{tabular}

Tabelle 30:Mittelwerte, Standardabweichungen und Korrelationen der Variablen in der integrativen Replikationsstudie innerhalb des Airplane-Szenarios.

Anmerkung: $\dagger p<.05$, einseitig; $* p<.05$, zweiseitig; $* * p<.01$, zweiseitig; $* * * p<.001$, zweiseitig. 


\subsection{Analyse der dichotomen Investitionsentscheidung}

Die Ergebnisse der logistischen Regressionsanalyse für die dichotome Investitionsentscheidung im Airplane-Szenario sind in Tabelle 31 dargestellt:

\begin{tabular}{lrrrrrr} 
& B & S.E. & Wald & df & Sig. & $\operatorname{Exp}(\mathrm{B})$ \\
\hline Sunk Cost Airplane & 4.32 & 4.61 & .88 & 1 & .349 & 74.91 \\
Action Orientation $(A O)$ & -.04 & .12 & .09 & 1 & .770 & .97 \\
Optimism vs. Pessimism (OP) & .16 & .14 & 1.32 & 1 & .251 & 1.17 \\
Regulatory Focus $(R F)$ & .78 & .48 & 2.64 & 1 & .104 & 2.18 \\
Rational Thinking Style (RTS) & -.52 & .96 & .30 & 1 & .587 & .59 \\
AO by Sunk Cost Airplane & .06 & .16 & .12 & 1 & .731 & 1.06 \\
OP by Sunk Cost Airplane & -.13 & .19 & .49 & 1 & .483 & .88 \\
RF by Sunk Cost Airplane & -.67 & .76 & .78 & 1 & .378 & .51 \\
RTS by Sunk Cost Airplane & -.02 & 1.30 & .00 & 1 & .986 & .98 \\
(Constant) & -.34 & 3.26 & .01 & 1 & .917 & .71 \\
\hline
\end{tabular}

Tabelle 31: Ergebnisse der simultanen logistischen Regressionsanalyse aller Prädiktoren; mit der dichotomen Investitionsentscheidung als Kriteriumsvariable im Airplane-Szenario.

Im Gegensatz zu den berichteten Einzelanalysen, konnte in dieser Analyse kein signifikanter Sunk-Cost-Effekt nachgewiesen werden. Allerdings investierten die Probanden bei vorhandenen versunkenen Kosten deskriptiv häufiger als in Abwesenheit versunkener Kosten $\left(M_{(S C+)}=88,0 \%\right.$ vs. $\left.M_{(S C-)}=15,1 \%\right)$, was dem Originalbefund für das AirplaneSzenario von Arkes und Blumer (1985) grundsätzlich entspricht. Vor allem aber zeigten sich darüber hinaus keine Interaktionseffekte zwischen den Persönlichkeitsvariablen und den versunkenen Kosten, die darauf hindeuten würden, dass von einer dieser Variablen ein moderierender Einfluss auf den Sunk-Cost-Effekt ausgehen könnte.

Abseits der zentralen Fragestellung fanden sich keine Haupteffekte für die vier Persönlichkeitsvariablen in der Form, dass Probanden mit einer bestimmten Persönlichkeit entweder signifikant mehr oder weniger investitionsfreudig gewesen wären. ${ }^{41}$

\footnotetext{
${ }^{41}$ Eine Analyse unter Verwendung der Subskalen RA und RE, anstelle der RTS Gesamtskala, erbrachte keine abweichenden Ergebnisse. Auch ein Einbezug der Variablen Alter, Geschlecht, Studiengang und Semesteranzahl als Kontrollvariablen oder eine Analyse aller 118 Probanden (mit den Item-Mittelwerten anstelle der fehlenden Werte in den Persönlichkeitsskalen) änderte nichts an der zentralen Befundlage.
} 


\subsection{Analyse der prozentualen Investitionsbereitschaft}

Die Ergebnisse der linearen Regressionsanalyse für die prozentuale Investitionsbereitschaft im Airplane-Szenario sind in Tabelle 32 aufgelistet:

\begin{tabular}{lrrrrr} 
& B & S.E. & $\beta$ & t & Sig. \\
\hline (Constant) & 52.66 & 24.16 & & 2.18 & .032 \\
Sunk Cost Airplane & 39.35 & 33.68 & .60 & 1.17 & .246 \\
Action Orientation $(A O)$ & .28 & 1.00 & .04 & 0.28 & .782 \\
\hline Optimism vs. Pessimism (OP) & .31 & 1.10 & .04 & 0.28 & .782 \\
\hline Regulatory Focus $($ RF) & 6.62 & 3.88 & .21 & 1.71 & .092 \\
Rational Thinking Style (RTS) & -8.34 & 6.96 & -.15 & -1.20 & .234 \\
AO by Sunk Cost Airplane & -.45 & 1.32 & -.04 & -.34 & .735 \\
OP by Sunk Cost Airplane & -.48 & 1.52 & -.04 & -.32 & .753 \\
RF by Sunk Cost Airplane & -3.92 & 5.86 & -.08 & -.67 & .505 \\
RTS by Sunk Cost Airplane & 2.58 & 9.51 & .14 & .27 & .787 \\
\hline
\end{tabular}

Tabelle 32: Ergebnisse der simultanen linearen Regressionsanalyse aller Prädiktoren; mit der prozentualen Investitionsbereitschaft als Kriteriumsvariable im Airplane-Szenario.

Auch in dieser Analyse zeigten sich die Probanden im Airplane-Szenario bei vorhandenen versunkenen Kosten investitionsfreudiger als in Abwesenheit versunkener Kosten $\left(M_{(S C+)}=\right.$ $71,3 \%$ vs. $\left.M_{(S C-)}=24,1 \%\right)$, jedoch wurde der Effekt wiederum nicht signifikant. Außerdem fanden sich auch an dieser Stelle keine Interaktionseffekte zwischen den Persönlichkeitsvariablen und den versunkenen Kosten. Auf Grundlage der beiden Analysen des Airplane-Szenarios können daher insgesamt keine Rückschlüsse auf einen moderierenden Einfluss der Persönlichkeitsvariablen auf den Sunk-Cost-Effekt gezogen werden.

Generelle Haupteffekte für die vier Persönlichkeitsvariablen blieben darüber hinaus ebenfalls aus. ${ }^{42}$

\footnotetext{
${ }^{42}$ Eine Analyse unter Verwendung der Subskalen RA und RE, anstelle der RTS Gesamtskala, erbrachte keine abweichenden Ergebnisse. Auch ein Einbezug der Variablen Alter, Geschlecht, Studiengang und Semesteranzahl als Kontrollvariablen oder eine Analyse aller 118 Probanden (mit den Item-Mittelwerten anstelle der fehlenden Werte in den Persönlichkeitsskalen) änderte nichts an der zentralen Befundlage.
} 


\subsubsection{Analysen des Druckerpressen-Szenarios}

Tabelle 33 liefert zunächst einen Überblick über die Mittelwerte, Standardabweichungen und Korrelationskoeffizienten aller Variablen aus den Analysen des DruckerpressenSzenarios in der integrativen Replikationsstudie. Unterschiede zu Tabelle 30 ergeben sich nur hinsichtlich der Variablen, die im unmittelbaren Zusammenhang zum DruckerpressenSzenario stehen; also beide Kriteriumsvariablen (dichotome Investitionsentscheidung und prozentuale Investitionsbereitschaft) sowie der Faktor versunkene Kosten. Die bereits bekannten Interkorrelationen der Persönlichkeitsvariablen werden nur der Vollständigkeit halber noch einmal aufgeführt.

Es zeigte sich auch in diesem Szenario eine signifikante Korrelation zwischen den versunkenen Kosten und der prozentualen Investitionsbereitschaft, die erwartungsgemäß negativ ausfiel und damit in die von Arkes und Blumer (1985) beschriebene Richtung weist. Die beiden Kriteriumsvariablen waren, wie schon im Airplane-Szenario, signifikant und positiv miteinander korreliert. Weiter fanden sich positive und signifikante Korrelationen für alle Persönlichkeitsvariablen mit den Kriteriumsvariablen (zum Teil nur einseitig), bis auf den Rational Thinking Style.

\begin{tabular}{llllllllll} 
& & $\mathrm{M}$ & $\mathrm{SD}$ & 1 & 2 & 3 & 4 & 5 & 6 \\
\hline 1. & Investition Printer & .75 & .44 & --- & & & & \\
2. & Willingness Printer & 64.50 & 27.23 & $.86^{* *}$ & --- & & & \\
3. & Sunk Cost Printer & .51 & .50 & -.16 & $-.23^{*}$ & --- & & \\
4. & Action Orientation & .00 & 4.52 & $.20^{*}$ & $.17^{\dagger}$ & -.10 & --- & & \\
5. & Optimism vs. Pessimism & .00 & 3.84 & $.19^{\dagger}$ & .09 & .01 & $.50^{* * *}$ & --- \\
6. & Regulatory Focus & .00 & 1.03 & $.28^{* *}$ & $.22^{*}$ & .01 & $.46^{* * *}$ & $.51^{* * *}$ & --- \\
7. & Rational Thinking Style & 3.50 & .59 & .14 & .09 & -.14 & $.40^{* * *}$ & $.31^{* *}$ & $.48^{* * *}$ \\
\hline
\end{tabular}

Tabelle 33:Mittelwerte, Standardabweichungen und Korrelationen der Variablen in der integrativen Replikationsstudie innerhalb des Druckerpressen-Szenarios.

Anmerkung: $\dagger p<.05$, einseitig; $* p<.05$, zweiseitig; ** $p<.01$, zweiseitig; $* * * p<.001$, zweiseitig. 


\subsection{Analyse der dichotomen Investitionsentscheidung}

Die Ergebnisse der logistischen Regressionsanalyse für die dichotome Investitionsentscheidung im Druckerpressen-Szenario sind in Tabelle 34 dargestellt:

\begin{tabular}{lrrrrrr} 
& B & S.E. & Wald & df & Sig. & $\operatorname{Exp}(\mathrm{B})$ \\
\hline Sunk Cost Printer & 1.55 & 3.71 & .18 & 1 & .676 & 4.71 \\
Action Orientation $(A O)$ & .02 & .10 & .03 & 1 & .853 & 1.02 \\
Optimism vs. Pessimism $(O P)$ & -.05 & .12 & .21 & 1 & .648 & .95 \\
Regulatory Focus $(R F)$ & .43 & .51 & .71 & 1 & .401 & 1.53 \\
Rational Thinking Style (RTS) & -.01 & .74 & .00 & 1 & .993 & .99 \\
AO by Sunk Cost Printer & .05 & .15 & .14 & 1 & .714 & 1.06 \\
OP by Sunk Cost Printer & .11 & .17 & .38 & 1 & .537 & 1.11 \\
RF by Sunk Cost Printer & .69 & .74 & .86 & 1 & .354 & 1.99 \\
RTS by Sunk Cost Printer & -.60 & 1.04 & .34 & 1 & .563 & .55 \\
(Constant) & 1.58 & 2.67 & .35 & 1 & .553 & 4.86 \\
\hline
\end{tabular}

Tabelle 34: Ergebnisse der simultanen logistischen Regressionsanalyse aller Prädiktoren; mit der dichotomen Investitionsentscheidung als Kriteriumsvariable im Druckerpressen-Szenario.

Wie bereits in den berichteten Einzelanalysen, konnte der grundlegende Sunk-CostHaupteffekt von Arkes und Blumer (1985) im Druckerpressen-Szenario für die dichotome Investitionsentscheidung auch in dieser Analyse nicht repliziert werden. Allerdings investierten die Probanden bei hohen versunkenen Kosten deskriptiv weniger als in Abwesenheit versunkener Kosten, was dem grundlegenden Befund von Arkes und Blumer tendenziell entspricht $\left(M_{(S C+)}=67,9 \%\right.$ vs. $\left.M_{(S C-)}=82,0 \%\right)$. Da sich darüber hinaus keine Interaktionen zwischen den Persönlichkeitsvariablen und den versunkenen Kosten zeigten, liegen auch an dieser Stelle keine Hinweise vor, dass von einer der Persönlichkeitsvariablen ein moderierender Einfluss auf den Sunk-Cost-Effekt ausgehen könnte.

Des Weiteren fanden sich keine Haupteffekte für die vier Persönlichkeitsvariablen, wonach Probanden mit einer bestimmten Persönlichkeit entweder signifikant mehr oder weniger investitionsfreudig innerhalb des Druckerpressen-Szenarios gewesen wären. ${ }^{43}$

\footnotetext{
${ }^{43}$ Eine Analyse unter Verwendung der Subskalen RA und RE, anstelle der RTS Gesamtskala, erbrachte keine abweichenden Ergebnisse. Auch ein Einbezug der Variablen Alter, Geschlecht, Studiengang und Semesteranzahl als Kontrollvariablen oder eine Analyse aller 118 Probanden (mit den Item-Mittelwerten anstelle der fehlenden Werte in den Persönlichkeitsskalen) änderte nichts an der zentralen Befundlage.
} 


\subsection{Analyse der prozentualen Investitionsbereitschaft}

Die Ergebnisse der linearen Regressionsanalyse für die prozentuale

Investitionsbereitschaft im Druckerpressen-Szenario sind in Tabelle 35 aufgelistet:

\begin{tabular}{lrrrrr} 
& B & S.E. & $\beta$ & t & Sig. \\
\hline (Constant) & 84.94 & 26.33 & & 3.23 & .002 \\
Sunk Cost Printer & 3.67 & 37.79 & .07 & .10 & .923 \\
Action Orientation (AO) & -.48 & .96 & -.08 & -.50 & .618 \\
\hline Optimism vs. Pessimism (OP) & -1.33 & 1.17 & -.19 & -1.14 & .256 \\
Regulatory Focus (RF) & 7.25 & 4.92 & .28 & 1.47 & .144 \\
Rational Thinking Style (RTS) & -3.85 & 7.27 & -.08 & -.53 & .598 \\
AO by Sunk Cost Printer & 2.23 & 1.48 & .27 & 1.51 & .135 \\
OP by Sunk Cost Printer & 1.29 & 1.70 & .14 & .76 & .450 \\
RF by Sunk Cost Printer & .33 & 6.57 & .01 & .05 & .961 \\
RTS by Sunk Cost Printer & -4.77 & 10.67 & -.31 & -.45 & .656 \\
\hline
\end{tabular}

Tabelle 35: Ergebnisse der simultanen linearen Regressionsanalyse aller Prädiktoren; mit der prozentualen Investitionsbereitschaft als Kriteriumsvariable im Druckerpressen-Szenario.

Im Gegensatz zu den berichteten Einzelanalysen fand sich kein signifikanter Haupteffekt für die versunkenen Kosten. Rein deskriptiv wies das Verhalten der Probanden aber nach wie vor in die von Arkes und Blumer (1985) beschriebene Richtung $\left(M_{(S C+)}=58,5 \%\right.$ vs. $M_{\left(S C_{-}\right)}=$ 70,9\%). Auch in dieser letzten Analyse der integrativen Replikationsstudie waren darüber hinaus keine signifikanten Interaktionen zwischen den Persönlichkeitsvariablen und den versunkenen Kosten feststellbar. Wie schon in den Analysen des Airplane-Szenarios fanden sich somit auch in den beiden Analysen des Druckerpressen-Szenarios insgesamt keinerlei Indizien, dass eine der vier Persönlichkeitsvariablen eine Moderatorvariable für den SunkCost-Effekt darstellen könnte.

Abseits der zentralen Fragestellung fanden sich, wie schon in den vorherigen Analysen, keine Haupteffekte für die vier Persönlichkeitsvariablen. ${ }^{44}$

\footnotetext{
${ }^{44}$ Eine Analyse unter Verwendung der Subskalen RA und RE, anstelle der RTS Gesamtskala, erbrachte einen signifikanten Haupteffekt für RE, $\beta=-.47, t[91]=-2.30, p=.023, r=-.02$. Dieser Nebenbefund wurde allerdings in der Originalstudie selbst nicht berichtet und bewegt sich abseits der zentralen Fragestellung der vorliegenden Arbeit. Ein Einbezug der Variablen Alter, Geschlecht, Studiengang und Semesteranzahl als Kontrollvariablen oder eine Analyse aller 118 Probanden (mit den Item-Mittelwerten anstelle der fehlenden Werte in den Persönlichkeitsskalen) änderte nichts an der zentralen Befundlage.
} 


\subsection{Diskussion}

Die vorliegende integrative Replikationsstudie stellte einen weiteren Versuch dar, die Originalbefunde von van Putten et al. (2010), Juliusson (2006), Molden und Hui (2011) sowie Wong et al. (2008) im Rahmen dieser Arbeit zu replizieren. Anders als in den direkten Replikationsstudien aus Kapitel 4, in denen die entsprechenden Persönlichkeitseffekte isoliert untersucht wurden, erfolgte in der integrativen Studie eine simultane Erhebung aller vier Persönlichkeitsvariablen. Dieses Vorgehen brachte den Vorteil mit sich, mögliche Interkorrelationen zwischen den Variablen aufdecken zu können und festzustellen, ob gewisse Persönlichkeitsvariablen ihren prädiktiven Wert einbüßen sobald die jeweils anderen Variablen ihrerseits einen Einfluss auf den Sunk-Cost-Effekt ausüben.

Es wurden die Ergebnisse zweier verschiedener Analysemethoden berichtet, nämlich erstens Einzelanalysen, bei denen wie in den Originalstudien jeweils nur eine der vier Persönlichkeitsvariablen betrachtet wurde, und zweitens integrative Analysen, in die alle Variablen simultan mit einbezogen wurden. Zwar zeigten sich in diesem Rahmen signifikante Interkorrelationen zwischen den Persönlichkeitsvariablen, allerdings waren in keiner aller angestellten Analysen Interaktionen zwischen den Persönlichkeitsvariablen und den versunkenen Kosten nachweisbar; weder in den Analysen des Airplane-Szenarios noch in den Analysen des Druckerpressen-Szenarios und zwar für keine der beiden erhobenen Kriteriumsvariablen (dichotome Investitionsentscheidung und prozentuale Investitionsbereitschaft). Insgesamt erscheint also auch auf Grundlage der Ergebnisse aus der integrativen Replikationsstudie zweifelhaft, dass von einer der vorgestellten Persönlichkeitsvariablen ein moderierender Einfluss auf den Sunk-Cost-Effekt ausgehen könnte.

Abgesehen davon konnten die grundlegenden Sunk-Cost-Haupteffekte von Arkes und Blumer (1985) zumindest in den Einzelanalysen für beide Sunk-Cost-Szenarien repliziert 
werden. Erst im Rahmen der simultanen Analyse, mit einer größeren Anzahl an Prädiktorvariablen, verschwanden die Sunk-Cost-Effekte, wiesen jedoch deskriptiv stets in die erwartete Richtung. Daher ist im Hinblick auf die Arbeit von van Putten et al. (2010) auch auf Grundlage der vorliegenden Ergebnisse von einem Vorzeichenfehler in ihren Berichten auszugehen. Hierin wurde nämlich innerhalb des Airplane-Szenarios ein negativer $B$ Regressionskoeffizient auswiesen, welcher im Gegensatz zu Arkes und Blumers (1985) grundlegenden Befund auf eine verminderte Investitionsneigung im Zuge steigender versunkener Kosten schließen ließe (vgl. Abschnitt 2.2.2.1).

Abseits der zentralen Fragestellung ließen sich auch nicht die berichteten Haupteffekte der Persönlichkeitsvariablen aus den jeweiligen Originalstudien replizieren. In Bezug auf Juliusson (2006) war der generelle Haupteffekt, wonach Optimisten eine stärkere Investitionsneigung aufwiesen als Pessimisten, in keiner der durchgeführten Analysen nachweisbar. Ein Haupteffekt fand sich hingegen in den Einzelanalysen für den regulatorischen Fokus, wonach Probanden mit steigenden Promotionwerten eine zunehmende Investitionsneigung im Airplane-Szenario zeigten. Allerdings steht dieser Befund im direkten Gegensatz zu Molden und Hui (2011), bei denen Personen mit einem Promotion Focus eigentlich die niedrigste Investitionsneigung aller Vergleichsbedingungen aufwiesen. Hinsichtlich des Rational Thinking Styles ließen sich keinerlei signifikante Haupteffekte der Rationalitätsskalen nachweisen, die bei Wong et al. (2008) in Form einer gesteigerten Investitionsbereitschaft für die RTS Gesamtskala sowie die RA Subskala berichtet wurden.

Insgesamt lässt sich festhalten, dass im Rahmen der integrativen Replikationsstudie in keiner aller durchgeführten Analysen die Befunde der jeweiligen Originalarbeiten repliziert werden konnten und sich keinerlei Indizien fanden, die auf einen moderierenden Einfluss der Persönlichkeitsvariablen auf den Sunk-Cost-Effekt schließen ließen. 


\section{6. Übergreifende Diskussion}

Der Sunk-Cost-Effekt beschreibt die Tendenz, ein Unterfangen umso wahrscheinlicher fortzusetzen, je mehr versunkene Kosten hierfür bereits entstanden sind (Arkes \& Blumer, 1985). Dieses irrationale Verhalten kann in der Praxis kostspielige Konsequenzen mit zum Teil hoher Tragweite nach sich ziehen. Daher wurden im Rahmen der vorliegenden Arbeit die Befunde verschiedener empirischer Arbeiten vorgestellt, welche nahelegen, dass die Persönlichkeitsvariablen Handlungs- und Lageorientierung (van Putten et al., 2010), Optimismus und Pessimismus (Juliusson, 2006), der regulatorische Fokus: Promotion und Prevention (Molden \& Hui, 2011) sowie der Rational Thinking Style (Wong et al., 2008) darüber mitbestimmen können, ob und wie anfällig Entscheidungsträger für den Sunk-CostEffekt sind. Sofern die berichteten Effekte zuträfen, ließen sich hieraus wertvolle Implikationen für die Praxis ableiten, wie zum Beispiel der Einsatz einer hierauf abgestimmten Personalauswahl bei der Besetzung von Schlüsselpositionen, in denen rationale Entscheidungen anstelle von Sunk-Cost-Effekten gefragt sind (vgl. Abschnitt 2.2.2).

Um derartige Empfehlungen für die Praxis aussprechen zu können, musste zunächst die grundsätzliche Belastbarkeit der Persönlichkeitseffekte sichergestellt werden, da diese bisher einzig durch ihre jeweiligen Originalstudien belegt sind. Wie in Abschnitt 2.3 erläutert, häuften sich in den letzten Jahren nämlich die Berichte darüber, dass viele aus der Literatur bekannte Befunde gar nicht replizierbar sind. Daher wurden, insbesondere vor dem Hintergrund der gegenwärtigen Replikationskrise, verschiedene Anstrengungen im Rahmen der vorliegenden Arbeit unternommen, um die Originalbefunde der vorgestellten Persönlichkeitsvariablen $\mathrm{zu}$ replizieren. Zum einen wurde eine Reihe direkter Replikationsstudien durchgeführt, in denen versucht wurde, alle Aspekte der jeweiligen Originalstudien im Sinne einer exakten Replikation bestmöglich nachzustellen (vgl. Kapitel 4). Zum anderen wurde eine integrative Replikationsstudie durchgeführt, in der alle 
Prädiktoren simultan erhoben wurden, um zu untersuchen, ob die entsprechenden Persönlichkeitseffekte auch nachweisbar sind, wenn die jeweils anderen Persönlichkeitsvariablen ihrerseits einen Einfluss ausüben (vgl. Kapitel 5).

Allerdings fanden sich in keiner aller durchgeführten Studien Indizien, dass von den hier untersuchten Persönlichkeitsvariablen eine moderierende Wirkung auf den Sunk-Cost-Effekt ausgehen könnte. Die vorliegenden Ergebnisse stellen stattdessen die grundsätzliche Belastbarkeit der Originalbefunde in Frage und legen nahe, dass bis zum jetzigen Zeitpunkt noch keine Persönlichkeitsvariablen in der Forschung identifiziert wurden, die zweifelsfrei einen Einfluss auf den Sunk-Cost-Effekt ausüben. Dennoch können die Befunde der Originalstudien nicht abschließend als falsch positiv deklariert werden. Dieses ließe sich allein auf Grundlage der hier vorgestellten Studien nicht mit hinreichender Sicherheit belegen. Ebenso wenig kann eindeutig gesagt werden, ob eine oder mehrere der in Abschnitt 2.3.1 dargelegten Ursachen, wie z.B. der Publication Bias, als Gründe für die nicht replizierbaren Originalbefunde herangezogen werden können. Für mögliche Erklärungen, warum eine solche Diskrepanz zwischen den Originalbefunden und denen der vorliegenden Arbeit besteht, sollen zunächst die Aspekte der vorliegenden Untersuchungen kritisch hinterfragt werden, bevor eine Gesamtwürdigung vorgenommen wird.

Eine denkbare Erklärung wäre, dass in den Untersuchungen der vorliegenden Arbeit falsch negative Befunde aufgetreten sind (vgl. Abschnitt 2.3.1.3). Dieser Annahme kann jedoch mit der Tatsache begegnet werden, dass zu jedem Persönlichkeitskonstrukt mindestens zwei Replikationsversuche durchgeführt wurden. Hierbei wurden in den direkten Replikationsstudien mindestens gleich große Stichprobenumfänge gewählt wie in den Originalstudien, in den meisten Fällen sogar größere. Die integrative Replikationsstudie erhöhte zudem kumulativ die Gesamtzahl aller Probanden, die pro Persönlichkeitskonstrukt untersucht wurden. Daher ist im Vergleich zu den Originalstudien näherungsweise von größeren Teststärken der vorliegenden Studien auszugehen, wodurch sich tatsächlich 
existierende Effekte theoretisch sogar mit einer erhöhten Wahrscheinlichkeit aufdecken ließen (vgl. Asendorpf et al., 2013; Bortz \& Schuster, 2010; Cohen, 1962). Dass dieses trotzdem in keinem Fall geglückt ist, spricht also eher dafür, dass die Originalbefunde wenig belastbar sind.

Ebenso könnten die unterschiedlichen Befundlagen auch auf eine Kontextabhängigkeit der Originaleffekte zurückzuführen sein. Zwar wurde insbesondere im Rahmen der direkten Replikationsstudien versucht, alle Aspekte aus den Originalstudien so gut wie möglich nachzubilden. Allerdings war dieses nur bis zu jenem Detailgrad realisierbar, der in den jeweiligen Originalstudien offengelegt wurde. Abweichungen hinsichtlich der Stichprobenzusammensetzungen, Untersuchungssituationen, Operationalisierungen oder Messzeitpunkte (vgl. Asendorpf et al., 2013) wurden weitestgehend vermieden oder aber in den jeweiligen Replikationsstudien explizit benannt, inklusive einer Darlegung, dass sich die Ergebnisse gegenüber diesen Abweichungen robust zeigten.

Eine gemeinsame Abweichung aller im Rahmen dieser Arbeit durchgeführten Replikationsstudien zu den Originalstudien ist allerdings, dass die Untersuchungen in Deutschland durchgeführt wurden. Hierdurch sind kulturell bedingte Verhaltensunterschiede der Probanden nicht auszuschließen (vgl. Henrich, Hein \& Norenzayan, 2010). Auch durch die Übersetzung des verwendeten Materials ins Deutsche könnten leichte Abweichungen hinsichtlich der Verständlichkeit aufgetreten sein. Leider wurden vor allem die Instruktionen in den Originalarbeiten nur relativ global oder gar nicht beschrieben, weswegen die Instruktionen der vorliegenden Arbeit nur sehr allgemein gehalten werden konnten. Es sei an dieser Stelle jedoch betont, dass Untersuchungsergebnisse von einer Vielzahl an Rahmenbedingungen beeinflusst werden können, die auch den Originalforschern selbst oft gar nicht bewusst sind - sogar von kleinen Variationen innerhalb desselben Laboratoriums. Die Idealvorstellung einer direkten Replikation ist aus diesem Grunde in der Forschungspraxis gar nicht realisierbar, weshalb strenggenommen nur von konzeptuellen Replikationsstudien 
gesprochen werden kann, die entweder mehr oder weniger direkt sind als andere (vgl. Asendorpf et al., 2013, Ijzerman, Brandt \& van Wolferen, 2013, Spellman, 2013). Sollte die Diskrepanz zwischen den Originalbefunden und denen der vorliegenden Replikationsstudien also auf Unterschiede zwischen den jeweiligen Rahmenbedingungen der Untersuchungen zurückzuführen sein, ergeben sich hierdurch dennoch insgesamt wertvolle Hinweise darauf, dass die vorgestellten Persönlichkeitseffekte, sofern sie im exakten Kontext der jeweiligen Originalstudie wirklich existieren sollten, vermutlich nicht sehr robust sein können, daher auch nicht generalisierbar sind und für eine Berücksichtigung in der Praxis umso eindeutiger nicht in Frage kommen.

Aus der Notwendigkeit heraus, in den Replikationsstudien alle Aspekte der jeweiligen Originalstudie bestmöglich zu übernehmen, wurde keine Incentivierung im Rahmen der Probandenvergütung vorgenommen. Daher wäre prinzipiell vorstellbar, dass die Probanden in den vorliegenden Untersuchungen, mit Ausblick auf die bereits feststehende Vergütung, teils beliebige Entscheidungen getroffen haben könnten und die Persönlichkeitseffekte auch deshalb nicht nachweisbar waren. Dieses erscheint jedoch als Erklärung für die Diskrepanzen unwahrscheinlich, da die grundlegenden Sunk-Cost-Effekte in den Szenarien von Arkes und Blumer (1985) weitestgehend repliziert werden konnten (abgesehen von einzelnen Analysen in der integrativen Replikationsstudie, in denen eine größere Zahl von Variablen mit einbezogen wurden). Die Ergebnisse aus den vorliegenden Untersuchungen erscheinen daher durchaus verlässlich im Sinne einer Vergleichbarkeit mit früherer Forschung, was darauf hinweist, dass das Probandenverhalten nicht willkürlich ausgefallen ist.

Abschließend sei noch einmal betont, dass auf Grundlage der gescheiterten Replikationsversuche dieser Arbeit nicht automatisch davon ausgegangen werden kann, dass die überprüften Originaleffekte nicht existieren würden. Die Ergebnisse dieser Arbeit können lediglich als Indizien dafür angesehen werden, dass es sich bei den vorgestellten Persönlichkeitseffekten um nicht replizierbare Befunde handelt - wenngleich die Evidenz zu 
Ungunsten dieser Persönlichkeitseffekte momentan stärker erscheint als die Evidenz zu Gunsten der Effekte. Die Ergebnisse der vorliegenden Arbeit stellen nur ein Teilstück eines Gesamtbildes dar, das erst durch eine Vielzahl von Replikationsstudien vervollständigt werden kann. Diese Arbeit sollte daher auch als Appell verstanden werden, in Zukunft weitere Replikationsversuche $\mathrm{zu}$ den vorgestellten Persönlichkeitseffekten $\mathrm{zu}$ unternehmen. Gleichzeitig sollten aber auch weitere aus der Literatur bekannte Effekte aus allen Forschungsgebieten einer kritischen Überprüfung unterzogen werden um noch mehr nicht replizierbare Befunde, die sich generell hartnäckig in der Literatur halten können, systematisch aufzudecken (vgl. Asendorpf et al., 2013; Greitemeyer, 2014; Simmons et al., 2011).

Replikationsstudien liefern in jedem Fachbereich einen wichtigen Erkenntnisgewinn, der zu einer Steigerung des wissenschaftlichen Wahrheitsgehalts führt. Aus diesem Grunde muss auch eine Nichtreplikation keineswegs ein gescheitertes Forschungsvorhaben darstellen. Entscheidend ist jedoch, dass die durchgeführten Replikationsversuche wirklich publiziert werden und der daraus resultierende Erkenntnisgewinn zur Vermeidung eines Publication Bias für jedermann zugänglich gemacht wird - so wie im Falle der vorliegenden Arbeit, die nahelegt, dass bisher keine Persönlichkeitsvariablen in der Forschung identifiziert wurden, die den Sunk-Cost-Effekt zweifelsfrei moderieren könnten. Es bietet sich nach aktuellem Wissensstand also nicht an, die untersuchten Persönlichkeitseffekte in der Praxis zu berücksichtigen, wenn das Auftreten irrationaler Sunk-Cost-Effekte wirksam vermieden werden sollen. 


\section{Literaturverzeichnis}

Arkes, H. R. \& Ayton, P. (1999). The sunk cost and concorde effects: Are humans less rational than lower animals?. Psychological Bulletin, 125(5), 591-600.

Arkes, H. R. \& Blumer, C. (1985). The psychology of sunk cost. Organizational Behavior and Human Decision Processes, 35(1), 124-140.

Arkes, H. R. \& Hutzel, L. (2000). The role of probability of success estimates in the sunk cost effekt. Journal of Behavioral Decision Making, 13(3), 295-306.

Asendorpf, J. B., Conner, M., De Fruyt, F., De Houwer, J., Denissen, J. J. A., Fiedler, K., Fiedler, S., Funder, D. C., Kliegl, R., Nosek, B. A., Perugini, M., Roberts, B. W., Schmitt, M., Vanaken, M. A. G., Weber, H. \& Wiecherts, J. M. (2013). Recommendations for increasing replicability in psychology. European Journal of Personality, 27(2), 108-119.

Bakker, M., Cramer, A. O. J., Matzke, D., Kievit, R. A., van der Maas, H. L. J., Wagenmakers, E. J. \& Borsboom, D. (2013). Dwelling on the Past. European Journal of Personality, 27(2), 120-121.

Bakker, M., van Dijk, A. \& Wicherts, J. M. (2012). The rules of the game called psychological science. Perspectives on Psychological Science, 7(6), 543-554.

Bem, D. J. (1987). Writing the empirical journal article. In J. M. Darley \& M. P. Zanna (Hrsg.), The compleat academic: A practical guide for the beginning social scientist (S. 171-201). Mahwah: Lawrence Erlbaum Associates.

Bem, D. J. (2011). Feeling the future: Experimental evidence for anomalous retroactive influences on cognition and affect. Journal of Personality and Social Psychology, 100(3), 407-425.

Bortz, J. \& Schuster, C. (2010). Statistik für Human- und Sozialwissenschaftler (7. Aufl.). Berlin: Springer-Verlag. 
Brockner, J. \& Rubin, J. Z. (1985). Entrapment in escalating conflicts: A social psychological analysis. New York: Springer.

Bruine de Bruin, W., Parker, A. M. \& Fischhoff, B. (2007). Individual differences in adult decision-making competence. Journal of Personality and Social Psychology, 92(5), 938956.

Cohen, J. (1962). The statistical power of abnormal-social psychological research: A review. Journal of Abnormal and Social Psychology, 65(3), 145-153.

Coleman, M. D. (2010). Sunk cost and commitment to medical threatment. Current Psychology, 29(2), 121-134.

Conlon, E. J. \& Parks, J. M. (1987). Information requests in the context of escalation. Journal of Applied Psychology, 72(3), 344-350.

Cooper, A. C., Woo, C. Y. \& Dunkelberg, W. C. (1988). Entrepreneurs' perceived chances for success. Journal of Business Venturing, 3(2), 97-108.

Cunha, M. \& Caldieraro, F. (2009). Sunk-cost effects on purely behavioral investments. Cognitive Science, 33(1), 105-113.

Dietrich, A. (1993). Planung und Entscheidung: Modelle-Ziele-Methoden; Mit Fallstudien und Lösungen (3. Aufl.). Wiesbaden: Gabler.

Epstein, S. (1994). Integration of the cognitive and the psychodynamic unconscious. American Psychologist, 49(8), 709-724.

Fanelli, D. (2010). "Positive" results increase down the hierarchy of the sciences. PLoS ONE, 5(4). Verfügbar unter:

http://journals.plos.org/plosone/article/file?id=10.1371/journal.pone. $0010068 \&$ type=printa ble [07.01.2017]. 
Fanelli, D. (2012). Negative results are disappearing from most disciplines and countries. Scientometrics, 90(3), 891-904.

Festinger, L. (1957). A theory of cognitive dissonance (2. Aufl.). Stanford: Stanford University Press.

Francis, G. (2012). Too good to be true: Publication Bias in two prominent studies from experimental psychology. Psychonomic Bulletin \& Review, 19(2), 151-156.

Francis, G. (2013). Replication, statistical consistency, and publication bias. Journal of Mathematical Psychology, 57(5), 153-169.

Freitas, A. L. \& Higgins, E. T. (2002). Enjoying goal-directed action: The role of regulatory fit. Psychological Science, 13(1), 1-6.

Freitas, A. L., Liberman, N. \& Higgins, E. T. (2002). Regulatory fit and resisting temptation during goal pursuit. Journal of Experimental Social Psychology, 38(3), 291-298.

Friedman, D., Pommerenke, K., Lukose, R., Milam, G. \& Huberman, B. A. (2007). Searching for the sunk cost fallacy. Experimental Economics, 10(1), 79-104.

Garland, H. (1990). Throwing good money after bad: The effect of sunk costs on the decision to escalate commitment to an ongoing project. Journal of Applied Psychology, 75(6), 728731.

Garland, H. \& Newport, S. (1991). Effects of absolute and relative sunk costs on the decision to persist with a course of action. Organizational Behavior and Human Decision Processes, 48(1), 55-69.

Gelman, A. \& Loken, E. (2013). The garden of forking paths: Why multiple comparisons can be a problem, even when there is no "fishing expedition" or "p-hacking" and the research hypothesis was posited ahead of time. Technical report, Department of Statistics, 
Columbia University, New York. Verfügbar unter:

http://www.stat.columbia.edu/ gelman/research/unpublished/p_hacking.pdf [07.01.2017].

Glaesmer, H., Hoyer, J., Klotsche, J. \& Herzberg, P. Y. (2008). Die deutsche Version des Life-Orientation-Tests (LOT-R) zum dispositionellen Optimismus und Pessimismus. Zeitschrift für Gesundheitspsychologie, 16(1), 26-31.

Gourville, J. T. \& Soman, D. (1998). Payment depreciation: The behavioral effects of temporally separating payments from consumption. Journal of Consumer Research, 25(2), $160-174$.

Greitemeyer, T. (2014). Article retracted, but the message lives on. Psychonomic Bulletin \& Review, 21(2), 557-561.

Greitemeyer, T., Schulz-Hardt, S., Popien, G. \& Frey, D. (2005). Der Einfluss versunkener monetärer und zeitlicher Kosten auf Ressourcenallokationen. Zeitschrift für Arbeits-und Organisationspsychologie, 49(1), 35-43.

Groß-Schuler, A. (2002). Irreversibilität und Unternehmensstrategie: Das Konzept der Sunk Costs und seine Entscheidungsrelevanz. Wiesbaden: DUV.

Hafenbrack, A. C., Kinias, Z. \& Barsade, S. G. (2014). Debiasing the mind through meditation: Mindfulness and the sunk-cost bias. Psychological Science, 25(2), 369-376.

Heath, C. (1995). Escalation and de-escalation of commitment in response to sunk costs: The role of budgeting in mental accounting. Organizational Behavior and Human Decision Processes, 62(1), 38-54.

Henrich, J., Heine, S. J. \& Norenzayan, A. (2010). The weirdest people in the world? Behavioral and Brain Sciences, 33(2), 61-135.

Hicks, D., Wouters, P., Waltman, L., De Rijcke, S. \& Rafols, I. (2015). Bibliometrics: the Leiden Manifesto for research metrics. Nature, 520, 429-431. 
Higgins, E. T. (1997). Beyond pleasure and pain. American Psychologist, 52(12), 1280-1300.

Higgins, E. T. (1998). Promotion and prevention: Regulatory focus as a motivational principle. In M. P. Zanna (Hrsg.), Advances in experimental social psychology (30, S. 146). New York: Academic Press.

Higgins, E. T., Friedman, R. S., Harlow, R. E., Idson, L. C., Ayduk, O. N. \& Taylor, A. (2001). Achievement orientations from subjective histories of success: Promotion pride versus prevention pride. European Journal of Social Psychology, 31(1), 3-23.

Higgins, E. T., Roney, C. J. R., Crowe, E. \& Hymes, C. (1994). Ideal versus ought predilections for approach and avoidance distinct self-regulatory systems. Journal of Personality and Social Psychology, 66(2), 276-286.

Hummel, S. \& Männel, W. (1986). Kostenrechnung 1: Grundlagen, Aufbau und Anwendung (4. Aufl.). Wiesbaden: Gabler.

Idson, L. C., Liberman, N. \& Higgins, E. T. (2004). Imagining how you'd feel: The role of motivational experiences from regulatory fit. Personality and Social Psychology Bulletin, 30(7), 926-937.

Ijzerman, H., Brandt, M. J. \& van Wolferen, J. (2013). Rejoice! In Replication. European Journal of Personality, 27(2), 128-129.

Ioannidis, J. P. A. (2005). Why most published findings are false. PLoS Medicine, 2(8), e124. Verfügbar unter: http://journals.plos.org/plosmedicine/article/file?id=10.1371/journal.pmed.0020124\&type $=$ printable [08.01.2017].

Ioannidis, J. P. A. \& Trikalinos, T. A. (2007). An exploratory test for an excess of significant findings. Clinical Trials, 4(3), 245-253. 
John, L. K., Loewenstein, G. \& Prelec, D. (2012). Measuring the prevalence of questionable research practices with incentives for truth telling. Psychological Science, 23(5), 524-532.

Johnstone, D. (2002). Behavioral and prescriptive explanations of a reverse sunk cost effect. Theory and decision, 53(3), 209-242.

Juliusson, A. (2006). Optimism as modifier of escalation of commitment. Scandinavian Journal of Psychology, 47(5), 345-348.

Juliusson, A., Karlsson, N. \& Gärling, T. (2005). Weighing the past and the future in decision making. European Journal of Cognitive Psychology, 17(4), 561-575.

Just, D. R. \& Wansink, B. (2011). The flat-rate pricing paradox: Conflicting effects of "allyou-can-eat" buffet pricing. The Review of Economics and Statistics, 93(1), 193-200.

Kahneman, D. \& Tversky, A. (1979). Prospect theory: An analysis of decision under risk. Econometrica: Journal of the Econometric Society, 47(2), 263-291.

Kahneman, D. \& Tversky, A. (1984). Choices, values, and frames. American Psychologist, 39(4), 341-350.

Karlsson, N., Juliusson, A., Grankvist, G. \& Gärling, T. (2002). Impact of decision goal on escalation. Acta Psychologica, 111(3), 309-322.

Keller, J. \& Bless, H. (2006). Regulatory fit and cognitive performance: The interactive effect of chronic and situationally induced self-regulatory mechanisms on test performance. European Journal of Social Psychology, 36(3), 393-405.

Klaczynski, P. A. \& Cottrell, J. M. (2004). A dual-process approach to cognitive development: The case of children's understanding of sunk cost decisions. Thinking \& Reasoning, 10(2), 147-174.

Krahnen, J. P. (1991). Sunk Costs und Unternehmensfinanzierung. Wiesbaden: Gabler. 
Krugmann, P. (2013). The Excel Depression. New York Times, 19. April 2013, S. A31. Verfügbar unter: http://www.nytimes.com/2013/04/19/opinion/krugman-the-exceldepression.html [07.01.2017]

Kuhl, J. (1994). A theory of action and state orientations. In Kuhl, J. \& Beckmann, J. (Hrsg.), Volition and personality: Action versus state orientation (S. 9-46). Seattle: Hogrefe \& Huber.

Kuhl, J. \& Beckmann, J. (1994a). Volition and personality: Action versus state orientation. Seattle: Hogrefe \& Huber.

Kuhl, J. \& Beckmann, J. (1994b). Action versus state orientation in the context of personality and volition. In Kuhl, J. \& Beckmann, J. (Hrsg.), Volition and personality: Action versus state orientation (S. 1-5). Seattle: Hogrefe \& Huber.

Kukla, A. (1974). Performance as a function of resultant achievement motivation (perceived ability) and perceived difficulty. Journal of Research in Personality, 7(4), 374-383.

Kunda, Z. (1990). The case for motivated reasoning. Psychological Bulletin, 108(3), 480-498.

Larrick, R. P., Morgan, J. N. \& Nisbett, R. E. (1990). Teaching the use of cost-benefit reasoning in everyday life. Psychological Science, 1(6), 362-370.

Lee, M. D. \& Wagenmakers, E. J. (2014). Bayesian cognitive modeling: A practical course. Cambridge: Cambridge University Press.

Liberman, N., Idson, L. C., Camacho, C. J. \& Higgins, E. T. (1999). Promotion and prevention choices between stability and change. Journal of personality and social psychology, 77(6), 1135-1145.

Liberman, N., Molden, D. C., Idson, L. C. \& Higgins, E. T. (2001). Promotion and prevention focus on alternative hypotheses: Implications for attributional functions. Journal of Personality and Social Psychology, 80(1), 5-18. 
Lockwood, P., Jordan, C. H. \& Kunda, Z. (2002). Motivation by positive or negative role models: Regulatory focus determines who will best inspire us. Journal of Personality and Social Psychology, 83(4), 854-864.

Makel, M. C., Plucker, J. A. \& Hegarty, B. (2012). Replications in psychology research: How often do they really occur?. Perspectives on Psychological Science, 7(6), 537-542.

Molden, D. C. \& Hui, C. M. (2011). Promoting de-escalation of commitment a regulatoryfocus perspective on sunk costs. Psychological Science, 22(1), 8-12.

Moon, H. (2001). Looking forward and looking back: Integrating completion and sunk-cost effects within an escalation-of-commitment progress decision. Journal of Applied Psychology, 86(1), 104-113.

Moosbrugger, H. \& Kelava, A. (2012). Testtheorie und Fragebogenkonstruktion (2. Aufl.). Berlin: Springer.

Nelson, L. D., Simmons, J. P. \& Simonsohn, U. (2012). Let's publish fewer papers. Psychological Inquiry, 23(3), 291-293.

Nosek, B. A., Spies, J. R. \& Motyl, M. (2012). Scientific utopia: II. Restructuring incentives and practices to promote truth over publishability. Perspectives on Psychological Science, $7(6), 615-631$.

Open Science Collaboration (2015). Estimating the reproducibility of psychological science. Science, 349(6251). Verfügbar unter: http://dx.doi.org/10.1126/science.aac4716 [06.01.2017].

Otto, A. R. (2010). Three attempts to replicate the behavioral sunk-cost effect: A note on Cunha and Caldieraro (2009). Cognitive Science, 34(8), 1379-1383. 
Pacini, R. \& Epstein, S. (1999). The relation of rational and experiential information processing styles to personality, basic beliefs, and the ratio-bias phenomenon. Journal of Personality and Social Psychology, 76(6), 972-987.

Pattison, K. F., Zentall, T. R. \& Watanabe, S. (2012). Sunk cost: Pigeons (Columba livia), too, show bias to complete a task rather than shift to another. Journal of Comparative Psychology, 126(1), 1-9.

Pindyck, R. S. \& Rubinfeld, D. I. (2009). Mikroökonomie (7. Aufl.). München: Pearson.

Prinz, F., Schlange, T. \& Asadullah, K. (2011). Believe it or not: How much can we rely on published data on potential drug targets? Nature Reviews Drug Discovery, 10, 712. Verfügbar unter: http://www.nature.com/nrd/journal/v10/n9/pdf/nrd3439-c1.pdf.

Rauner, M. (2014). Dieser Mann hat der Wissenschaft die Smarties geklaut. ZEIT Wissen, 2014(4). Verfügbar unter: http://www.zeit.de/zeit-wissen/2014/04/hochstapler-betrugwissenschaft [03.02.2015].

Robbert, T., Roth, S. \& Straus, L. (2013). Sunk-Costs und die Nutzung von Dienstleistungen. In S. Roth (Hrsg.), Aktuelle Beiträge zur Dienstleistungsforschung (S. 29-47). Wiesbaden: Springer Fachmedien.

Reinhart, C. \& Rogoff, K. (2010). Growth in a time of dept. American Economic Review 100(2), 573-578.

Rosenthal, R. (1979). The file drawer problem and tolerance for null results. Psychological Bulletin, 86(3), 638-641.

Ross, J. \& Staw, B. M. (1993). Organizational escalation and exit: Lessons from the Shoreham nuclear power plant. Academy of Management Journal, 36(4), 701-732.

Schaub, H. (1997). Sunk Costs, Rationalität und ökonomische Theorie. Stuttgart: SchäfferPoeschel. 
Scheier, M. F., Carver, C. S. \& Bridges, M. W. (1994). Distinguishing optimism from neuroticism (and trait anxiety, self-mastery and self-esteem): A reevaluation of the Life Orientation Test. Journal of Personality and Social Psychology, 67(6), 1063-1078.

Schmidt, J. B. \& Calantone, R. J. (1998). Are really new product development projects harder to shut down?. Journal of Product Innovation Management, 15(2), 111-123.

Schmidt-Atzert, L. \& Amelang, M. (2012). Psychologische Diagnostik (5. Aufl.). Berlin: Springer.

Schuh, B. (2014). Fälschung in der Wissenschaft - Manuskript: Vertrauen naiv, Kontrolle fatal. Deutschlandfunk - Wissenschaft im Brennpunkt. Verfügbar unter: http://www.deutschlandfunk.de/faelschung-in-der-wissenschaft-manuskript-vertrauennaiv.740.de.html?dram:article_id=284241 [03.02.2015].

Schulz-Hardt, S. \& Frey, D. (1999). Sind wir unfähig, Verluste zu stoppen? Eine kritische Bestandsaufnahme der Entrapment-Forschung. In W. Hacker \& M. Rinck (Hrsg.), Zukunft gestalten - Bericht über der 41. Kongress der Deutschen Gesellschaft für Psychologie in Dresden, 1998 (S. 487-496). Lengerich: Pabst.

Schulz-Hardt, S., Vogelgesang, F. \& Mojzisch, A. (2007). Finanzpsychologie. In K. Moser (Hrsg.), Wirtschaftspsychologie (S.193-264). Springer: Heidelberg.

Simmons, J. P., Nelson, L. D. \& Simonsohn, U. (2011). False-positive psychology: Undisclosed flexibility in data collection and analysis allows presenting anything as significant. Psychological Science, 22(11), 1359-1366.

Sleesman, D. J., Conlon, D. E., McNamara, G. \& Miles, J. E. (2012). Cleaning up the big muddy: A meta-analytic review of the determinants of escalation of commitment. Academy of Management Journal, 55(3), 541-562. 
Smith, C. M., Tindale, R. S. \& Steiner, L. (1998). Investment decisions by individuals and groups in 'sunk cost' situations: The potential impact of shared representations. Group Processes and Intergroup Relations, 1(2), 175-189.

Soman, D. (2001). The mental accounting of sunk time costs: Why time is not like money. Journal of Behavioral Decision Making, 14(3), 169-185.

Soman, D. \& Cheema, A. (2001). The effect of windfall gains on the sunk-cost effect. Marketing Letters, 12(1), 51-62.

Soman, D. \& Gourville, J. T. (2001). Transaction decoupling: How price bundling affects the decision to consume. Journal of Marketing Research, 38(1), 30-44.

Spellman, B.A. (2013). There is no such thing as replication, but we should do it anyway. European Journal of Personality, 27(2), 136-137.

Staw, B. M. (1976). Knee-deep in the big muddy: A study of escalating commitment to a chosen course of action. Organizational Behavior and Human Performance, 16(1), 27-44.

Staw, B. M. (1981). The escalation of commitment to a course of action. Academy of Management Review, 6(4), 577-587.

Staw, B. M. (1997). The escalation of commitment: An update and appraisal. In Z. Shapira (Hrsg.), Organizational decision making (S. 191-215). Cambridge: Cambridge University Press.

Staw, B. M. \& Hoang, H. (1995). Sunk costs in the NBA: Why draft order affects playing time and survival in professional basketball. Administrative Science Quarterly, 40(3), 474494.

Strough, J., Mehta, C. M., McFall, J. P. \& Schuller, K. L. (2008). Are older adults less subject to the sunk-cost fallacy than younger adults?. Psychological Science, 19(7), 650-652. 
Strough, J., Schlosnagle, L., Karns, T., Lemaster, P. \& Pichayayothin, N. (2014). No time to waste: Restricting life-span temporal horizons decreases the sunk-cost fallacy. Journal of Behavioral Decision Making, 27(1), 78-94.

Tan, H. T. \& Yates, J. F. (1995). Sunk cost effects: The influence of instruction and future return estimates. Organizational Behavior and Human Decision Processes, 63(3), 311319.

Thaler, R. H. (1980). Toward a positive theory of consumer choice. Journal of Economic Behavior and Organization, 1(1), 39-60.

Thaler, R. H. (1985). Mental accounting and consumer choice. Marketing Science, 4(3), 199214.

Thaler, R. H. (1999). Mental accounting matters. Journal of Behavioral Decision Making, 12(3), 183-206.

Thames, E. A. (1996). The sunk-cost effect: The importance of context. Journal of Social Behavior and Personality, 11(4), 817-826.

Van Putten, M., Zeelenberg, M. \& van Dijk, E. (2009). Dealing with missed opportunities: Action vs. state orientation moderates inaction inertia. Journal of Experimental Social Psychology, 45(4), 808-815.

Van Putten, M., Zeelenberg, M. \& van Dijk, E. (2010). Who throws good money after bad? Action vs. state orientation moderates the sunk cost fallacy. Judgment and Decision Making, 5(1), 33-36.

Wagenmakers, E. J. (2012). A year of horrors. De Psychonoom, 27, 12-13.

Wong, K. F. E., Kwong, J. Y. Y. \& Ng, C. K. (2008). When thinking rationally increases biases: The role of rational thinking style in escalation of commitment. Applied Psychology, 57(2), 246-271. 
Zeelenberg, M. \& van Dijk, E. (1997). A reverse sunk cost effect in risky decision making:

Sometimes we have too much invested to gamble. Journal of Economic Psychology, 18(6), 677-691. 


\section{Tabellenverzeichnis}

Tabelle 1: Befunde der Originalstudie von van Putten et al. (2010). Die dichotome Investitionsentscheidung stellte hier die Kriteriumsvariable dar.

Tabelle 2: Anzahl der Probanden in der Originalstudie von van Putten et al. (2010), die sich für eine

Folgeinvestition entschieden

Tabelle 3: Anzahl der Probanden in der vorliegenden Replikationsstudie, die sich für eine Folgeinvestition entschieden.

Tabelle 4: Ergebnisse der Varianzanalysen von Juliusson (2006) mit der dichotomen Investitionsentscheidung und dem Präferenzindex als abhängige Variablen.

Tabelle 5: Deskriptive Ergebnisse von Juliusson (2006): Prozentuale Investitionshäufigkeiten und Präferenzindizes in den Versuchsbedingungen, aufgeteilt nach den Stufen des induzierten Optimismus vs. Pessimismus, der versunkenen Kosten und der Gewinnwahrscheinlichkeit.

Tabelle 6: Deskriptive Ergebnisse der ersten Replikationsstudie zu Juliusson (2006): Prozentuale Investitionshäufigkeiten und Präferenzindizes in den Versuchsbedingungen, aufgeteilt nach den Stufen des induzierten Optimismus vs. Pessimismus, der versunkenen Kosten und der Gewinnwahrscheinlichkeit.

Tabelle 7: Deskriptive Ergebnisse der zweiten Replikationsstudie zu Juliusson (2006): Prozentuale Investitionshäufigkeiten und Präferenzindizes in den Versuchsbedingungen, aufgeteilt nach den Stufen des induzierten Optimismus vs. Pessimismus, der versunkenen Kosten und der Gewinnwahrscheinlichkeit.

Tabelle 8: Befunde aus den Analysen von Molden und Hui (2011) mit der Investitionsentscheidung als abhängige Variable

Tabelle 9: Mittelwerte, Standardabweichungen und Korrelationen der Variablen bei Wong et al. (2008).

Tabelle 10: Mittelwerte, Standardabweichungen und Korrelationen der Variablen in der Replikationsstudie..... 90

Tabelle 11: Resultate der hierarchischen Regressionsanalysen von Wong et al. (2008) und der Replikationsstudie im Vergleich. Dargestellt werden die standardisierten Beta-Koeffizienten, die Kriteriumsvariable ist jeweils die prozentuale Investitionsbereitschaft.

Tabelle 12: Mittelwerte, Standardabweichungen und Korrelationen der Variablen im erweiterten Design der 
Tabelle 13: Resultate der hierarchischen Regressionsanalysen des erweiterten Designs der Replikationsstudie. Dargestellt werden die standardisierten Beta-Koeffizienten, die Kriteriumsvariable ist die prozentuale Investitionsbereitschaft. 93

Tabelle 14: Ergebnisse der logistischen Regressionsanalyse des Prädiktors Handlungs- und Lageorientierung; mit der dichotomen Investitionsentscheidung als Kriteriumsvariable im Airplane-Szenario.

Tabelle 15: Ergebnisse der linearen Regressionsanalyse des Prädiktors Handlungs- und Lageorientierung; mit der prozentualen Investitionsbereitschaft als Kriteriumsvariable im Airplane-Szenario.

Tabelle 16: Ergebnisse der logistischen Regressionsanalyse des Prädiktors Handlungs- und Lageorientierung; mit der dichotomen Investitionsentscheidung als Kriteriumsvariable im Druckerpressen-Szenario. 109

Tabelle 17: Ergebnisse der linearen Regressionsanalyse des Prädiktors Handlungs- und Lageorientierung; mit der prozentualen Investitionsbereitschaft als Kriteriumsvariable im Druckerpressen-Szenario.

Tabelle 18: Ergebnisse der logistischen Regressionsanalyse des Prädiktors Optimismus und Pessimismus; mit der dichotomen Investitionsentscheidung als Kriteriumsvariable im Airplane-Szenario.

Tabelle 19: Ergebnisse der linearen Regressionsanalyse des Prädiktors Optimismus und Pessimismus; mit der prozentualen Investitionsbereitschaft als Kriteriumsvariable im Airplane-Szenario.

Tabelle 20: Ergebnisse der logistischen Regressionsanalyse des Prädiktors Optimismus und Pessimismus; mit der dichotomen Investitionsentscheidung als Kriteriumsvariable im Druckerpressen-Szenario.....

Tabelle 21: Ergebnisse der linearen Regressionsanalyse des Prädiktors Optimismus und Pessimismus; mit der prozentualen Investitionsbereitschaft als Kriteriumsvariable im Druckerpressen-Szenario.

Tabelle 22: Ergebnisse der logistischen Regressionsanalyse des Prädiktors regulatorischer Fokus; mit der dichotomen Investitionsentscheidung als Kriteriumsvariable im Airplane-Szenario.

Tabelle 23: Ergebnisse der linearen Regressionsanalyse des Prädiktors regulatorischer Fokus; mit der prozentualen Investitionsbereitschaft als Kriteriumsvariable im Airplane-Szenario.

Tabelle 24: Ergebnisse der logistischen Regressionsanalyse des Prädiktors regulatorischer Fokus; mit der dichotomen Investitionsentscheidung als Kriteriumsvariable im Druckerpressen-Szenario.

Tabelle 25: Ergebnisse der linearen Regressionsanalyse des Prädiktors regulatorischer Fokus; mit der prozentualen Investitionsbereitschaft als Kriteriumsvariable im Druckerpressen-Szenario.

Tabelle 26: Ergebnisse der logistischen Regressionsanalyse des Prädiktors Rational Thinking Style; mit der dichotomen Investitionsentscheidung als Kriteriumsvariable im Airplane-Szenario.

Tabelle 27: Ergebnisse der linearen Regressionsanalyse des Prädiktors Rational Thinking Style; mit der prozentualen Investitionsbereitschaft als Kriteriumsvariable im Airplane-Szenario. 
Tabelle 28: Ergebnisse der logistischen Regressionsanalyse des Prädiktors Rational Thinking Style; mit der dichotomen Investitionsentscheidung als Kriteriumsvariable im Druckerpressen-Szenario.

Tabelle 29: Ergebnisse der linearen Regressionsanalyse des Prädiktors Rational Thinking Style; mit der prozentualen Investitionsbereitschaft als Kriteriumsvariable im Druckerpressen-Szenario. ....

Tabelle 30:Mittelwerte, Standardabweichungen und Korrelationen der Variablen in der integrativen

Replikationsstudie innerhalb des Airplane-Szenarios. Anmerkung: $\uparrow \mathrm{p}<.05$, einseitig; $* \mathrm{p}<.05$, zweiseitig; $* * \mathrm{p}<.01$, zweiseitig; $* * * \mathrm{p}<.001$, zweiseitig

Tabelle 31: Ergebnisse der simultanen logistischen Regressionsanalyse aller Prädiktoren; mit der dichotomen Investitionsentscheidung als Kriteriumsvariable im Airplane-Szenario.

Tabelle 32: Ergebnisse der simultanen linearen Regressionsanalyse aller Prädiktoren; mit der prozentualen

Investitionsbereitschaft als Kriteriumsvariable im Airplane-Szenario.

Tabelle 33:Mittelwerte, Standardabweichungen und Korrelationen der Variablen in der integrativen

Replikationsstudie innerhalb des Druckerpressen-Szenarios. Anmerkung: $\uparrow \mathrm{p}<.05$, einseitig; * $\mathrm{p}<$

.05 , zweiseitig; $* * \mathrm{p}<.01$, zweiseitig; $* * * \mathrm{p}<.001$, zweiseitig.

Tabelle 34: Ergebnisse der simultanen logistischen Regressionsanalyse aller Prädiktoren; mit der dichotomen Investitionsentscheidung als Kriteriumsvariable im Druckerpressen-Szenario.

Tabelle 35: Ergebnisse der simultanen linearen Regressionsanalyse aller Prädiktoren; mit der prozentualen Investitionsbereitschaft als Kriteriumsvariable im Druckerpressen-Szenario.

Tabelle 36: Regressionsanalyse der $\mathrm{SC}^{(+)}$-Bedingungen zur Prüfung möglicher Reihenfolgeeffekte; mit der Investitionsbereitschaft als Kriteriumsvariable (vgl. Abschnitt 4.4.2 - Model 1a).....

Tabelle 37: Regressionsanalyse der $\mathrm{SC}^{(+)}$-Bedingungen zur Prüfung möglicher Reihenfolgeeffekte; mit der Investitionsbereitschaft als Kriteriumsvariable (vgl. Abschnitt 4.4.2 - Model 1b).

Tabelle 38: Regressionsanalyse auf Basis des erweiterten Designs zur Prüfung möglicher Reihenfolgeeffekte; mit der Investitionsbereitschaft als Kriteriumsvariable (vgl. Abschnitt 4.4.2 - Model 2a). XLII

Tabelle 39: Regressionsanalyse auf Basis des erweiterten Designs zur Prüfung möglicher Reihenfolgeeffekte; mit der Investitionsbereitschaft als Kriteriumsvariable (vgl. Abschnitt 4.4.2 - Model 2b). XLII 


\section{Abbildungsverzeichnis}

Abbildung 1: Simple-Slope-Analysen der Originalstudie: Vergleich der B-Koeffizienten (logarithmierte Odds Ratios) der UV „,versunkene Kosten“ für Personen mit hohen und niedrigen Ausprägungen der Handlungs- und Lageorientierung.

Abbildung 2: Simple-Slope-Analysen der Replikationsstudie: Vergleich der B-Koeffizienten (logarithmierte

Odds Ratios) der UV „,versunkene Kosten“ für Personen mit hohen und niedrigen Ausprägungen der Handlungs- und Lageorientierung.

Abbildung 3: Vergleich der Ergebnisse aus Moldens und Huis (2011) Originalstudie (links) mit denen aus der vorliegenden Replikationsstudie (rechts). Auf der Ordinate ist die durchschnittliche Investitionsrate in Prozent abgetragen, die Abszisse ist nach den drei Motivationsstufen unterteilt.

Abbildung 4: Vergleich der durchschnittlichen prozentualen Investitionsraten aller Versuchsbedingungen aus der vorliegenden Replikationsstudie, unterteilt nach den drei Stufen des regulatorischen Fokus sowie beiden Stufen der versunkenen Kosten $\left(\mathrm{grau}=\mathrm{SC}^{(+)}\right.$, schwarz $\left.=\mathrm{SC}^{(-)}\right)$

Abbildung 5: Vergleich der durchschnittlichen prozentualen Investitionsraten aller Versuchsbedingungen aus der zweiten Replikationsstudie, unterteilt nach den drei Stufen des regulatorischen Fokus sowie beiden Stufen der versunkenen Kosten $\left(\mathrm{grau}=\mathrm{SC}^{(+)}\right.$, $\left.\operatorname{schwarz}=\mathrm{SC}^{(-)}\right)$. 81 
Anhang 
Anhang A: Versuchsmaterial 


\section{Aiplane-Szenario von Arkes und Blumer (1985)}

Das Airplane-Szenario von Arkes und Blumer (1985) wurde im Rahmen der vorliegenden

Arbeit in verschiedenen Studien eingesetzt. Dabei wurde in der Regel die Investitionsentscheidung mit einer dichotomen Antwortmöglichkeit (ja vs. nein) abgefragt, im Rahmen der integrativen Replikationsstudie erfolgte zusätzlich auch eine Abfrage der prozentualen Investitionsbereitschaft.

\section{* Die $\mathrm{SC}^{(+)}$-Version des Szenarios lautete wie folgt:}

„Als der Präsident einer Fluggesellschaft hast Du 9 Millionen Euro des Unternehmens in ein Entwicklungsprojekt investiert. Ziel war es, ein Flugzeug zu bauen, das vom herkömmlichen Radar nicht erfasst wird, sozusagen ein „,radarunsichtbares“ Flugzeug. Insgesamt waren Investitionen von 10 Millionen Euro für das Projekt eingeplant. Als das Projekt zu 90\% fertig gestellt ist, beginnt eine andere Firma, ein Flugzeug zu bewerben, welches nicht vom Radar erfasst werden kann. Außerdem ist es offensichtlich, dass deren Flugzeug wesentlich schneller und sehr viel ökonomischer ist als das Flugzeug, das Dein Unternehmen baut.

a) Investierst Du die 1 Mio. Euro, um das Projekt fertig zu stellen?

b) Wie groß ist Deine Bereitschaft (in \%) dieses Projekt fertig zu stellen?“

* Die $\mathrm{SC}^{(-)}-$Version des Szenarios lautete wie folgt:

„Als der Präsident einer Fluggesellschaft hast Du einen Vorschlag von einem Deiner Angestellten erhalten. Der Vorschlag beinhaltet, die letzten 1 Millionen Euro Deines Entwicklungsvermögens zu nutzen, um ein Flugzeug zu entwickeln, das vom herkömmlichen Radar nicht erfasst wird, sozusagen ein „radarunsichtbares“ Flugzeug. Jedoch hat gerade eine andere Firma begonnen, ein Flugzeug zu bewerben, welches nicht vom Radar erfasst werden kann. Außerdem ist es offensichtlich, dass deren Flugzeug wesentlich schneller und sehr viel ökonomischer ist als das Flugzeug, das Dein Unternehmen bauen könnte.

a) Investierst Du die 1 Mio. Euro, um das Projekt zu realisieren?

b) Wie groß ist Deine Bereitschaft (in \%) dieses Projekt zu realisieren?“ 


\section{Druckerpressen-Szenario von Arkes und Blumer (1985)}

Das Druckerpressen-Szenario von Arkes und Blumer (1985) wurde im Rahmen der vorliegenden Arbeit ebenfalls in verschiedenen Studien eingesetzt. Auch hierin wurde die Investitionsentscheidung mit einer dichotomen Antwortmöglichkeit (ja vs. nein) abgefragt, während im Rahmen der integrativen Replikationsstudie zusätzlich auch eine Abfrage der prozentualen Investitionsbereitschaft erfolgte.

\section{* Die $\mathrm{SC}^{(+)}$-Version des Szenarios lautete wie folgt:}

„Du bist Eigentümer einer Druckerei und willst Deinen Betrieb modernisieren.

Du musst Dich entscheiden, ob Du die dafür veranschlagten $200.000 €$ für eine neue Druckerpresse oder für neue Transporter ausgeben willst. Du entscheidest Dich, die Druckerpresse zu kaufen, die zu ungefähr denselben Kosten doppelt so schnell wie Deine alte Druckerpresse arbeitet.

Eine Woche nach dem Kauf der neuen Druckerpresse geht einer Deiner Konkurrenten bankrott. Um schnell zu Geld zu kommen bietet er Dir an, seine computergesteuerte Druckerpresse für $10.000 €$ an Dich zu verkaufen.

Diese Presse arbeitet 50\% schneller als die, die Du gerade neu erworben hast und das zu ungefähr der Hälfte der Kosten. Du weißt, dass Du Deine gerade erworbene Presse nicht verkaufen kannst um dieses Geld zu bekommen, weil sie speziell für Deine Ansprüche gebaut wurde und nicht modifiziert werden kann. Allerdings hast Du $10.000 €$ Ersparnisse zur Verfügung.

a) Kaufst Du die computergesteuerte Druckerpresse von Deinem bankrotten Konkurrenten?

b) Wie groß ist Deine Bereitschaft (in \%) die Druckerpresse zu kaufen?“

\section{* Die $\mathrm{SC}^{(-)}-$Version des Szenarios lautete wie folgt:}

„Du bist Eigentümer einer Druckerei und willst Deinen Betrieb modernisieren.

Du musst Dich entscheiden, ob Du die dafür veranschlagten $200.000 €$ für eine neue Druckerpresse oder für neue Transporter ausgeben willst. Du entscheidest Dich, die Transporter zu kaufen, die zu ungefähr denselben Kosten Deine Produkte doppelt so schnell ausliefern können wie Deine alten Transporter.

Eine Woche nach dem Kauf der neuen Transporter geht einer Deiner Konkurrenten bankrott. Um schnell zu Geld zu kommen bietet er Dir an, seine computergesteuerte Druckerpresse für $10.000 €$ an Dich zu verkaufen. Diese Presse arbeitet 50\% schneller als Deine bisherige Presse und das zu ungefähr der Hälfte der Kosten. Du weißt, dass Du Deine alte Presse nicht verkaufen kannst um dieses Geld zu bekommen, weil sie speziell für Deine Ansprüche gebaut wurde und nicht modifiziert werden kann. Allerdings hast Du $10.000 €$ Ersparnisse zur Verfügung.

a) Kaufst Du die computergesteuerte Druckerpresse von Deinem bankrotten Konkurrenten?

b) Wie groß ist Deine Bereitschaft (in \%) die Druckerpresse zu kaufen?“ 


\section{Sunk-Cost-Material von Juliusson (2006)}

Die vier Sunk-Cost-Szenarien in der Arbeit zu Optimismus und Pessimismus wurden von Juliusson (2006) selbst erstellt. Eine Beschaffung des Originalmaterials war leider nicht mehr möglich. Daher wurde versucht, die Szenarien auf Basis der Ausführungen des Artikels so gut wie möglich zu rekonstruieren. Die Manipulation von Optimismus und Pessimismus erfolgte inter-individuell, die der versunkenen Kosten (hoch vs. niedrig) und der Gewinnwahrscheinlichkeit (exakt vs. ungefähr) intra-individuell.

* Zur Manipulation von Optimismus lautete die $\mathrm{SC}^{(+)}$-Version des Szenarios mit einer exakten Gewinnwahrscheinlichkeitsangabe wie folgt:

„Bitte stellen Sie sich folgende Situation vor:

Sie haben sich vor einiger Zeit entschieden, in die Entwicklung eines neuen Produkts zu investieren. Von diesem neuen Produkt war zu erwarten, dass es wettbewerbsfähig am Markt sein würde und dementsprechend auch Gewinne erzielen kann. Die Wahrscheinlichkeit für Gewinne betrug zum Zeitpunkt Ihrer Investition $20 \%$.

Um das Projekt zu verwirklichen, stand Ihnen ein begrenztes Budget in Höhe von 10.000€ zur Verfügung, wovon Sie bereits $\mathbf{5 0 0 0 €}$ in die Entwicklung des Produkts investiert haben.

Heute erfahren Sie, dass bei der Entwicklung des Produkts alles wie erwartet verlaufen ist. Einziges Manko ist, dass die Kosten der Entwicklung unterschätzt wurden und nun weitere $5000 €$ notwendig wären, um die Entwicklung erfolgreich abschließen zu können.

Sie müssen sich nun entscheiden, ob Sie weitere 5000€ in das Projekt investieren möchten, oder nicht investieren und somit das Projekt abbrechen. Brechen Sie ab, ist das Geld Ihrer ersten Investition verloren.

Mit dem Produkt könnten Sie später Gewinne erzielen, die Ihre gesamten Investitionskosten übersteigen. Die tatsächliche Wahrscheinlichkeit für eine Gewinnerzielung wurde inzwischen revidiert und liegt jetzt bei $\mathbf{5 0 \%}$ (ursprünglich 20\%).“

* In der SC $\mathrm{SC}^{(-)}$-Version wurde der Betrag der versunkenen Kosten auf $\mathbf{1 7 0 0 €}$ geändert, in der Version mit einer ungefähren Gewinnwahrscheinlichkeit, lautete der letzte Satz „,(..) und liegt jetzt zwischen 30\% und 70\%“. Zur Manipulation von Pessimismus betrug der Wert der ursprünglichen Gewinnwahrscheinlichkeit im ersten und letzten Abschnitt jeweils $80 \%$. 


\section{Sunk-Cost-Material von Wong et al. (2008)}

Das von Wong et al. (2008) verwendete Szenario, welches an Arkes und Blumers (1985) Airplane-Szenario angelehnt war, wurde im Rahmen der vorliegenden Arbeit ins Deutsche übersetzt und um eine äquivalente $\mathrm{SC}^{(-)}$-Version ergänzt. In Kombination mit der Variation positiven und negativen Framings, die von Wong et al. (2008) als Kontrollvariable miterhoben wurde, ergaben sich vier verschiedene Szenarioversionen.

\section{* Die $\mathrm{SC}^{(+)}$-Version des Szenarios mit negativem Framing lautete wie folgt:}

„Du bist der Vizepräsident für den operativen Bereich eines mittelständischen Unternehmens, welches HighTech Produkte herstellt. Du hast 5 Millionen Euro in ein Forschungsprojekt investiert, für das 10 Millionen Euro als Budget festgelegt waren. In dem Projekt soll eine Vorrichtung zur Radarverschlüsselung entwickelt werden, durch die Schiffe von einem herkömmlichen Radar nicht mehr auffindbar sind (also ein radarunsichtbares Schiff). Die technische Abteilung hat Dich informiert, dass das Projekt zu 90\% abgeschlossen ist. Du erwartest, dass das Unternehmen 600 Millionen Euro einnehmen wird, nachdem das Produkt auf dem Markt erhältlich ist.

Allerdings hast $\mathrm{Du}$ gerade herausgefunden, dass eine andere Firma bereits begonnen hat, ein gleichartiges Produkt zu vermarkten, mit einem viel besseren Design: Es benötigt weniger Platz und ist viel einfacher zu bedienen als Dein Design.

Du stehst nun vor der Entscheidung, entweder (a) das Projekt abzubrechen und das restliche Geld in ein anderes neues Produkt zu investieren oder (b) die nächste 1 Million Euro zu bewilligen, um das derzeitige Projekt fortzuführen.

Von der Marketingabteilung (ihre Einschätzung ist äußerst präzise) erhältst Du folgende Informationen:

a. Falls Du Dich entscheidest das Projekt abzubrechen und den Rest des Geldes in ein neues Produkt investierst, ist es zu 100\% sicher, dass Du 400 Millionen Euro Verlust haben wirst, verglichen mit Deiner ursprünglichen Einschätzung des aktuellen Projekts.

b. Falls Du Dich entscheidest das Projekt fortzuführen, hast Du eine 1/3 Chance keine Verluste zu machen und eine 2/3 Chance 600 Millionen Euro Verlust zu machen, verglichen mit Deiner ursprünglichen Einschätzung des aktuellen Projekts.

Wie groß ist Deine Bereitschaft (in \%) dieses Projekt fortzuführen?“

* In der positiven Framing-Version wurde der letzte Absatz wie folgt geändert:

„Von der Marketingabteilung (ihre Einschätzung ist äußerst präzise) erhältst Du folgende Informationen:

a. Falls Du Dich entscheidest das Projekt abzubrechen und den Rest des Geldes in ein neues Produkt investierst, ist es zu 100\% sicher, dass Du 200 Millionen Euro Gewinn haben wirst.

b. Falls Du Dich entscheidest das Projekt fortzuführen, hast Du eine 1/3 Chance die kompletten 600 Millionen Euro als Gewinn zu erzielen und eine 2/3 Chance keinen Gewinn zu erzielen.“ 


\section{* Die SC ${ }^{(-)}$-Version lautete in Anlehnung an Arkes und Blumer (1985) wie folgt:}

„Du bist der Vizepräsident für den operativen Bereich eines mittelständischen Unternehmens, welches HighTech Produkte herstellt und erhältst einen Vorschlag von einem Deiner Angestellten. Der Vorschlag beinhaltet, 1 Million Euro Deines allgemeinen Entwicklungsbudgets, in dem noch 5 Millionen Euro verfügbar sind, zu nutzen, um eine Vorrichtung zur Radarverschlüsselung zu entwickeln, durch die Schiffe von einem herkömmlichen Radar nicht mehr auffindbar sind (also ein radarunsichtbares Schiff). Du erwartest, dass das Unternehmen 600 Millionen Euro einnehmen wird, nachdem das Produkt auf dem Markt erhältlich ist.

Allerdings hast $\mathrm{Du}$ gerade herausgefunden, dass eine andere Firma bereits begonnen hat, ein gleichartiges Produkt zu vermarkten, mit einem viel besseren Design: Es benötigt weniger Platz und ist viel einfacher zu bedienen als Dein Design.

Du stehst nun vor der Entscheidung, entweder (a) das Projekt nicht zu starten und das restliche Geld in ein anderes neues Produkt zu investieren oder (b) die 1 Million Euro zu bewilligen, um das vorgeschlagene Projekt zu realisieren.

Von der Marketingabteilung (ihre Einschätzung ist äußerst präzise) erhältst Du folgende Informationen:

a. Falls Du Dich entscheidest das Projekt nicht zu starten und den Rest des Geldes in ein neues Produkt investierst, ist es zu 100\% sicher, dass Du 400 Millionen Euro Verlust haben wirst, verglichen mit Deiner ursprünglichen Einschätzung des aktuellen Projekts.

b. Falls Du Dich entscheidest das Projekt zu realisieren, hast Du eine 1/3 Chance keine Verluste zu machen und eine 2/3 Chance 600 Millionen Euro Verlust zu machen, verglichen mit Deiner ursprünglichen Einschätzung des aktuellen Projekts.

Wie groß ist Deine Bereitschaft (in \%) dieses Projekt zu realisieren?“

* In der positiven Framing-Version lautete der letzte Absatz wie folgt:

„Von der Marketingabteilung (ihre Einschätzung ist äußerst präzise) erhältst Du folgende Informationen:

a. Falls Du Dich entscheidest das Projekt nicht zu starten und den Rest des Geldes in ein neues Produkt investierst, ist es zu 100\% sicher, dass Du 200 Millionen Euro Gewinn erzielen wirst.

b. Falls Du Dich entscheidest das Projekt zu realisieren, hast Du eine 1/3 Chance die kompletten 600 Millionen Euro als Gewinn zu erzielen und eine 2/3 Chance keinen Gewinn zu erzielen.“ 


\section{HAKEMP 90 (Kuhl \& Beckmann, 1994a)}

Zur Messung der Handlungs- und Lageorientierung auf Trait-Ebene wurden von van Putten et al. (2010) 24 Items aus dem ACS-90 Fragebogen eingesetzt. Im Rahmen der vorliegenden Arbeit wurden die entsprechenden Items aus dem HAKEMP 90, der deutschsprachigen Version des ACS-90, verwendet (Kuhl \& Beckmann, 1994a):

1. Wenn ich etwas Wertvolles verloren habe und jede Suche vergeblich war, dann

A. $\square$ kann ich mich schlecht auf etwas anderes konzentrieren.

B. $\square$ denke ich nicht mehr lange darüber nach.

2. Wenn ich weiß, dass etwas bald erledigt werden muss, dann

A. $\square$ muss ich mir oft einen Ruck geben, um den Anfang zu kriegen.

B. $\square$ fällt es mir leicht, es schnell hinter mich zu bringen.

3. Wenn ich vier Wochen lang an einer Sache gearbeitet habe und dann doch alles misslungen ist, dann

A. $\square$ dauert es lange, bis ich mich damit abfinde

B. $\square$ denke ich nicht mehr lange darüber nach.

4. Wenn ich nichts Besonderes vorhabe und Langeweile habe, dann

A. $\square$ kann ich mich manchmal nicht entscheiden, was ich tun soll.

B. $\square$ habe ich meist rasch eine neue Beschäftigung.

5. Wenn ich bei einem Wettkampf öfter hintereinander verloren habe, dann

A. $\square$ denke ich bald nicht mehr daran.

B. $\square$ geht mir das noch eine ganze Weile durch den Kopf.

6. Wenn ich ein schwieriges Problem angehen will, dann

A. $\square$ kommt mir die Sache vorher wie ein Berg vor.

B. $\square$ überlege ich, wie ich die Sache auf eine einigermaßen angenehme Weise hinter mich bringen kann.

7. Wenn mir ein neues Gerät versehentlich auf den Boden gefallen und nicht mehr zu reparieren ist, dann

A. $\square$ finde ich mich rasch mit der Sache ab.

B. $\square$ komme ich nicht so schnell darüber hinweg.

8. Wenn ich ein schwieriges Problem lösen muss, dann

A. $\square$ lege ich meist sofort los.

B. $\square$ gehen mir zuerst andere Dinge durch den Kopf, bevor ich mich richtig an die Aufgabe heranmache.

9. Wenn ich jemanden, mit dem ich etwas Wichtiges besprechen muss, wiederholt nicht zu Hause antreffe, dann

A. $\square$ geht mir das oft durch den Kopf, auch wenn ich mich schon mit etwas anderem beschäftige.

B. $\square$ blende ich das aus, bis die nächste Gelegenheit kommt, ihn zu treffen.

10. Wenn ich vor der Frage stehe, was ich in einigen freien Stunden tun soll, dann

A. $\square$ überlege ich manchmal eine Weile, bis ich mich entscheiden kann.

B. $\square$ entscheide ich mich meist ohne Schwierigkeit für eine der möglichen Beschäftigungen. 
11. Wenn ich nach einem Einkauf zu Hause merke, dass ich zu viel bezahlt habe, aber das Geld nicht mehr zurückbekomme,

A. $\square$ fällt es mir schwer, mich auf irgendetwas anderes zu konzentrieren.

B. $\square$ fällt es mir leicht, die Sache auszublenden.

12. Wenn ich eigentlich zu Hause arbeiten müsste, dann

A. $\square$ fällt es mir oft schwer, mich an die Arbeit zu machen.

B. $\square$ fange ich meist ohne weiteres an.

13. Wenn meine Arbeit als völlig unzureichend bezeichnet wird, dann

A. $\square$ lasse ich mich davon nicht lange beirren.

B. $\square$ bin ich zuerst wie gelähmt.

14. Wenn ich sehr viele wichtige Dinge zu erledigen habe, dann

A. $\square$ überlege ich oft, wo ich anfangen soll.

B. $\square$ fällt es mir leicht, einen Plan zu machen und ihn auszuführen.

15. Wenn ich mich verfahre (z. B. mit dem Auto, mit dem Bus usw.) und eine wichtige Verabredung verpasse, dann

A. $\square$ kann ich mich zuerst schlecht aufraffen, irgendetwas anderes anzupacken.

B. $\square$ lasse ich die Sache erst mal auf sich beruhen und wende mich ohne Schwierigkeiten anderen Dingen zu.

16. Wenn ich zu zwei Dingen große Lust habe, die ich aber nicht beide machen kann, dann

A. $\square$ beginne ich schnell mit einer Sache und denke gar nicht mehr an die andere.

B. $\square$ fällt es mir nicht so leicht, von einer der beiden Sachen ganz Abstand zu nehmen.

17. Wenn mir etwas ganz Wichtiges immer wieder nicht gelingen will, dann

A. $\square$ verliere ich allmählich den Mut.

B. $\square$ vergesse ich es zunächst einmal und beschäftige mich mit anderen Dingen.

18. Wenn ich etwas Wichtiges, aber Unangenehmes zu erledigen habe, dann

A. $\square$ lege ich meist sofort los.

B. $\square$ kann es eine Weile dauern, bis ich mich dazu aufraffe.

19. Wenn mich etwas traurig macht, dann

A. $\square$ fällt es mir schwer, irgendetwas anderes zu tun.

B. $\square$ fällt es mir leicht, mich durch andere Dinge abzulenken.

20. Wenn ich vorhabe, eine umfassende Arbeit zu erledigen, dann

A. $\square$ denke ich manchmal zu lange nach, womit ich anfangen soll.

B. $\square$ habe ich keine Probleme loszulegen.

21. Wenn einmal sehr viele Dinge am selben Tag misslingen, dann

A. $\square$ weiß ich manchmal nichts mit mir anzufangen.

B. $\square$ bleibe ich fast genauso tatkräftig, als wäre nichts passiert.

22. Wenn ich vor einer langweiligen Aufgabe stehe, dann

A. $\square$ habe ich meist keine Probleme, mich an die Arbeit zu machen.

B. $\square$ bin ich manchmal wie gelähmt.

23. Wenn ich meinen ganzen Ehrgeiz darin gesetzt habe, eine bestimmte Arbeit gut zu verrichten und es geht schief, dann

A. $\square$ kann ich die Sache auf sich beruhen lassen und mich anderen Dingen zuwenden.

B. $\square$ fällt es mir schwer, überhaupt noch etwas zu tun.

24. Wenn ich unbedingt einer lästigen Pflicht nachgehen muss, dann

A. $\square$ bringe ich die Sachen ohne Schwierigkeiten hinter mich.

B. $\square$ fällt es mir schwer, damit anzufangen. 


\section{LOT-R (Scheier et al., 1994)}

Die deutsche Übersetzung des LOT-R von Scheier et al. (1994) stammte von Glaesmer et al. (2008) und wurde im Rahmen dieser Arbeit zur Messung von Optimismus und Pessimismus auf Trait-Ebene eingesetzt. Die Items, deren Zustimmung auf einer fünfstufigen

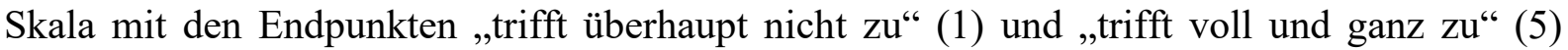
abgefragt wurde, lauteten wie folgt:

1. Auch in ungewissen Zeiten erwarte ich normalerweise das Beste.

2. Es fällt mir leicht, mich zu entspannen.

3. Wenn bei mir etwas schief laufen kann, dann tut es das auch.

4. Meine Zukunft sehe ich immer optimistisch.

5. In meinem Freundeskreis fühle ich mich wohl.

6. Es ist wichtig für mich, ständig beschäftigt zu sein.

7. Fast nie entwickeln sich die Dinge nach meinen Vorstellungen.

8. Ich bin nicht allzu leicht aus der Fassung zu bringen.

9. Ich zähle selten darauf, dass mir etwas Gutes widerfährt.

10. Alles in allem erwarte ich, dass mir mehr gute als schlechte Dinge widerfahren.

Optimismus Items: $1,4,10$

Pessimismus Items: 3, 7, 9

Füll-Items: 2, 5, 6, 8 


\section{Lockwood Skala (Lockwood et al., 2002)}

Die deutsche Übersetzung der Lockwood-Skala von Lockwood et al. (2002) stammte von Keller und Bless (2006) und wurde im Rahmen dieser Arbeit zur Messung des regulatorischen Fokus auf Trait-Ebene eingesetzt. Die Items, deren Zustimmung auf einer siebenstufigen Skala mit den Endpunkten „trifft überhaupt nicht zu“(1) und „trifft voll und ganz zu“ (7) abgefragt wurde, lauteten wie folgt:

1. Im Allgemeinen konzentriere ich mich darauf, negative Ereignisse in meinem Leben zu vermeiden.

2. Ich mache mir Sorgen, meinen Verantwortungen und Pflichten nicht gerecht zu werden.

3. Ich stelle mir oft vor, wie ich meine Hoffnungen und Ziele erreichen werde.

4. Ich denke oft über die Person nach, die ich befürchte in der Zukunft einmal werden zu können.

5. Ich denke oft über die Person nach, die ich idealerweise in der Zukunft einmal werden möchte.

6. Üblicherweise konzentriere ich mich auf den Erfolg, den ich hoffe in der Zukunft zu erreichen.

7. Ich mache mir oft Sorgen, dass ich es nicht schaffen werde, meine akademischen Ziele zu verwirklichen.

8. Ich denke oft daran, wie ich akademischen Erfolg erreichen werde.

9. Ich stelle mir oft vor, wie ich schlimme Erfahrungen mache, von denen ich befürchte, dass sie in der Zukunft auf mich zukommen könnten.

10. Ich denke oft darüber nach, wie ich Misserfolge in meinem Leben vermeiden kann.

11. Ich bin stärker darauf ausgerichtet, Verluste zu vermeiden als Gewinne zu erzielen.

12. Momentan ist mein wichtigstes Ziel im Studium, meine akademischen Ambitionen zu erfüllen.

13. Momentan besteht mein wichtigstes Ziel im Studium darin, es zu vermeiden, in Prüfungen, Klausuren und Seminararbeiten zu versagen.

14. Ich sehe mich selbst als jemanden, der hauptsächlich danach strebt, sein ideales Selbstbild zu erreichen seine Hoffnungen, Wünsche und Ziele.

15. Ich sehe mich selbst als jemanden, der hauptsächlich danach strebt, die an ihn gestellten Erwartungen zu erfüllen - den Pflichten, Verantwortungen und Zwängen gerecht zu werden.

16. Im Allgemeinen bin ich darauf konzentriert, positive Ergebnisse in meinem Leben zu erreichen.

17. Ich stelle mir oft vor, wie ich schöne Erfahrungen mache, von denen ich hoffe, dass sie in der Zukunft auf mich zukommen.

18. Im Großen und Ganzen bin ich stärker darauf ausgerichtet, Gewinne zu erzielen als Verluste zu vermeiden.

Promotion Items: 3, 5, 6, 8, 12, 14, 16, 17, 18

Prevention Items: 1, 2, 4, 7, 9, 10, 11, 13, 15 


\section{Rationality Scale (Pacini \& Epstein, 1999)}

Die 20 von Wong et al. (2008) verwendeten Items zur Messung des Rational Thinking Styles wurden ins Deutsche übersetzt und ihre jeweilige Zustimmungstendenz auf einer fünfstufigen Skala mit den Endpunkten „trifft überhaupt nicht zu“(1) und ,trifft voll und ganz zu“ (5) abgefragt. Die Items, die sich zur Hälfte auf Rational Ability und Rational Engagement bezogen, waren entweder positiv oder negativ formuliert:

1. (-) Ich versuche Situationen zu vermeiden, die gründliches Nachdenken über etwas erfordern.

2. (-) Ich kann nicht gut komplizierte Probleme lösen.

3. (+) Ich genieße intellektuelle Herausforderungen.

4. (-) Ich bin nicht besonders gut im Lösen von Problemen, die eine sorgfältige logische Analyse erfordern.

5. (-) Ich mag es nicht viel nachdenken zu müssen.

6. (+) Ich habe Spaß am Lösen von Problemen, die angestrengtes Nachdenken erfordern.

7. (-) Nachdenken ist nicht meine Vorstellung von einer angenehmen Tätigkeit.

8. (-) Ich bin kein besonders analytischer Denker.

9. (-) Das sorgfältige Durchdenken von Dingen ist keine meiner Stärken.

10. (+) Ich bevorzuge komplizierte Probleme gegenüber einfachen Problemen.

11. (-) Lange angestrengt über etwas nachzudenken gibt mir wenig Befriedigung.

12. (-) Unter Druck schlussfolgere ich nicht gut.

13. (+) Ich kann Dinge viel besser logisch herausfinden als die meisten anderen Leute.

14. (+) Ich habe einen logischen Verstand.

15. (+) Ich habe Spaß daran in abstrakten Begriffen zu denken.

16. (+) Ich habe kein Problem damit Dinge sorgfältig zu durchdenken.

17. (+) Das Benutzen von Logik ist für gewöhnlich ein geeignetes Mittel für mich um Probleme in meinem Leben zu begreifen.

18. (-) Die Antwort zu kennen, ohne den Grund dahinter verstehen zu müssen, ist ausreichend für mich.

19. (+) Ich habe gewöhnlich erklärbare Gründe für meine Entscheidungen.

20. (+) Neue Denkweisen zu lernen wäre sehr reizvoll für mich.

Rational Ability Items: 2, 4, 8, 9, 12, 13, 14, 16, 17, 19

Rational Engagement Items: 1, 3, 5, 6, 7, 10, 11, 15, 18, 20

Etwaiges über diese Arbeit hinausgehendes Material kann beim Autor angefragt werden. 
Anhang B: Ergänzende Analysen 


\section{Manipulationskontrolle des regulatorischen Fokus}

Im Rahmen der zweiten direkten Replikationsstudie zu Molden und Hui (2011) wurde der Versuch einer Manipulationskontrolle integriert. Hierbei handelte es sich um eine explorative Ergänzung, da in der Originalstudie kein vergleichbarer Versuch berichtet wurde. Auch in einer Reihe weiterer hochrangig publizierter Studien, bei denen der regulatorische Fokus in der gleichen Art und Weise experimentell induziert wurde wie bei Molden und Hui (2011), wurde keine explizite Manipulationskontrolle berichtet, weshalb es sich hierbei also um ein gängiges Vorgehen in dieser Forschung handelt (vgl. z.B. Freitas \& Higgins, 2002; Freitas et al., 2002; Higgins et al., 1994; Idson et al., 2004; Liberman et al., 1999; Liberman et al., 2001).

Für die Manipulationskontrolle in dieser Arbeit wurde der chronische regulatorische Fokus direkt nach der experimentellen Manipulation mithilfe der Lockwood-Skala (vgl. Lockwood et al. 2002) erhoben, um somit ein näherungsweises Manipulationsmaß zu erhalten. Die deutsche Übersetzung von Keller und Bless (2006) enthielt jeweils neun Items zum Promotion Focus und zum Prevention Focus auf Trait-Ebene. Die Probanden kreuzten dabei auf einer siebenstufigen Skala mit den Endpunkten 1 (überhaupt nicht zutreffend) bis 7 (vollkommen zutreffend) ihre jeweilige Zustimmungstendenz zu den Fragen an. Für die Analyse wurden daraufhin die Mittelwerte der jeweiligen Skalen für Promotion und Prevention bestimmt. Diese Vorgehensweise erfolgte wie erwähnt explorativ und in Kenntnis darüber, dass eine Trait-Skala kein sensitives Maß für eine State-Untersuchung darstellen muss.

Zur Bestimmung ob die Manipulation geglückt ist, wurden einfaktorielle Varianzanalysen durchgeführt, mit dem dreistufigen Faktor der induzierten Motivation (Promotion vs. Prevention vs. Control). Als abhängige Variablen dienten jeweils die berechneten TraitSkalen für Promotion und Prevention, mit Ausprägungen zwischen 1 (Minimum) und 7 
(Maximum). Im Fall einer erfolgreichen Manipulation sollten Probanden mit einem induzierten Promotion-Focus signifikant höhere Trait-Promotion-Werte aufweisen, als Probanden der entsprechenden Vergleichsbedingungen. Gleiches gilt für den induzierten Prevention Focus in Verbindung mit den Trait-Prevention-Werten.

Jedoch zeigte sich weder für Promotion $\left(F[2,242]=.52, p=.595, \eta_{\mathrm{p}}^{2}=.00\right)$ noch für Prevention $\left(F[2,242]=1.35, p=.262, \eta_{\mathrm{p}}^{2}=.01\right)$ ein entsprechender Haupteffekt. Auch in direkten Vergleichen der Motivationsbedingungen untereinander zeigten sich keine signifikanten Unterschiede, weder für Trait-Promotion $\left(M_{(\text {Promotion })}=4.92\right.$ vs. $M_{(\text {Control })}=4.78$, $F[1,160]=.83, p=.365, \eta_{\mathrm{p}}^{2}=.01 ; M_{(\text {Promotion })}=4.92$ vs. $M_{(\text {Prevention })}=4.91, F[1,158]=.00$, $\left.p=.962, \eta_{\mathrm{p}}^{2}=.00\right)$ noch für Trait-Prevention $\left(M_{(\text {Prevention })}=4.04\right.$ vs. $M_{(\text {Control })}=4.17$, $F[1,160]=.73, p=.395, \eta_{\mathrm{p}}^{2}=.01 ; M_{(\text {Prevention })}=4.04$ vs. $M_{(\text {Promotion })}=3.90, F[1,158]=.64$, $\left.p=.426, \eta_{\mathrm{p}}^{2}=.00\right)$.

Die vorliegenden Ergebnisse könnten darauf hindeuten, dass die Manipulation des regulatorischen Fokus möglicherweise nicht erfolgreich war. Allerdings ist wie erwähnt unklar, ob die Trait-Ausprägungen auf der Lockwood-Skala überhaupt ein sensitives Maß für den regulatorischen Fokus auf State-Ebene darstellen. Da bei Molden und Hui (2011) außerdem gar keine Manipulationskontrolle berichtet wurde, ist ebenso unbekannt, ob die Manipulation überhaupt in der Originalstudie geglückt ist, bzw. anhand des hier beschriebenen Verfahrens bestätigt worden wäre. Es existiert somit keine Basis für einen Vergleich der Manipulationserfolge in beiden Studien, weshalb diesbezüglich von einer Diskussion im Rahmen dieser Arbeit abgesehen wird. 


\section{Überprüfung von Reihenfolgeeffekten im Rahmen der Untersuchung}

\section{des Rational Thinking Styles}

Im Rahmen der vorliegenden Arbeit wurde es als methodisch besser betrachtet, zuerst die Trait-Ausprägung des Rational Thinking Styles und erst im Anschluss die Investitionsbereitschaft als abhängige Variable zu erheben, so wie es in den übrigen Studien der vorgestellten Persönlichkeitsvariablen ebenfalls der Fall war. Da TraitPersönlichkeitsvariablen zeitlich stabil sind (vgl. Abschnitt 2.2.2), sollte die umgekehrte Erhebungsreihenfolge kein Problem darstellen. Dennoch wurde, um die Vergleichbarkeit zur Originalstudie nicht in Frage zu stellen, eine Vorstudie mit 80 Teilnehmern durchgeführt, in der entweder zuerst der Rationalitätsfragebogen oder das Sunk-Cost-Szenario präsentiert wurde. Das durchschnittliche Alter der Teilnehmer betrug 20,74 Jahre, in einer Spanne zwischen 18 und 32 Jahren. 53 Probanden waren weiblich (66\%) und 27 männlich (34\%).

Zur Überprüfung, ob die Erhebungsreihenfolge einen Einfluss auf die berichteten Effekte hat, wurden verschiedene Regressionsanalysen mit den drei Skalen des Rational Thinking Styles und der Reihenfolge als zweistufige Kontrollvariable durchgeführt - einerseits auf Basis der reinen $\mathrm{SC}^{(+)}$-Bedingungen wie in der Originalstudie und andererseits auf Basis des erweiterten Designs, in das wie in den Replikationsstudien eine $\mathrm{SC}^{(-)}$-Faktorstufe integriert wurde. Jedoch haben sich in keiner der angestellten Analysen signifikante Haupteffekte oder Interaktionen für die Reihenfolge ergeben. Aus diesem Grund erschien es gerechtfertigt, die Erhebungen im Rahmen der Replikationsstudien dieser Arbeit in der eingangs beschriebenen Reihenfolge durchzuführen. Die folgenden Tabellen zeigen die Ergebnisse der durchgeführten Kontrollanalysen. ${ }^{45}$

\footnotetext{
${ }^{45}$ In den Tabellen steht die Abkürzung „IE“ für einen Interaktionseffekt mit den versunkenen Kosten (SC) und den Skalen des Rational Thinking Styles (RTS, RA \& RE), wie jeweils angeben.
} 
Analysen auf Basis der $S C^{(+)}$-Bedingungen, wie in der Originalstudie:

\begin{tabular}{|c|c|c|c|c|c|}
\hline & \multicolumn{2}{|c|}{ Unstandardized Coefficients } & \multirow{2}{*}{$\begin{array}{c}\text { Standardized } \\
\text { Coefficients } \\
\text { Beta } \\
\end{array}$} & \multirow[b]{2}{*}{$\mathrm{t}$} & \multirow[b]{2}{*}{ Sig. } \\
\hline & $\mathrm{B}$ & Std. Error & & & \\
\hline (Constant) & 139.69 & 60.62 & & 2.30 & .028 \\
\hline Verantwortlichkeit & 2.21 & 3.59 & .12 & .62 & .543 \\
\hline Alter & -2.71 & 1.73 & -.26 & -1.57 & .127 \\
\hline Geschlecht & 4.94 & 10.05 & .09 & .49 & .627 \\
\hline Framing & -16.07 & 8.79 & -.29 & -1.83 & .077 \\
\hline$R T S$ & -8.96 & 12.49 & -.15 & -.72 & .478 \\
\hline Reihenfolge & -125.47 & 78.64 & -2.26 & -1.60 & .120 \\
\hline IE: RTS $x$ Reihenfolge & 33.10 & 21.35 & 2.18 & 1.55 & .131 \\
\hline
\end{tabular}

Tabelle 36: Regressionsanalyse der $S^{(+)}$-Bedingungen zur Prüfung möglicher Reihenfolgeeffekte; mit der Investitionsbereitschaft als Kriteriumsvariable (vgl. Abschnitt 4.4 .2 - Model 1a).

\begin{tabular}{|c|c|c|c|c|c|}
\hline & \multicolumn{2}{|c|}{ Unstandardized Coefficients } & \multirow{2}{*}{$\begin{array}{c}\text { Standardized } \\
\text { Coefficients } \\
\text { Beta }\end{array}$} & \multirow[b]{2}{*}{$\mathrm{t}$} & \multirow[b]{2}{*}{ Sig. } \\
\hline & $\mathrm{B}$ & Std. Error & & & \\
\hline (Constant) & 135.65 & 60.98 & & 2.22 & .034 \\
\hline Verantwortlichkeit & 2.07 & 3.67 & .11 & .56 & .577 \\
\hline Alter & -2.56 & 1.76 & -.25 & -1.45 & .157 \\
\hline Geschlecht & 5.26 & 10.11 & .09 & .52 & .606 \\
\hline Framing & -17.62 & 8.94 & -.32 & -1.97 & .058 \\
\hline$R A$ & -2.51 & 17.09 & -.04 & -.15 & .884 \\
\hline$R E$ & -5.87 & 16.58 & -.11 & -.35 & .726 \\
\hline Reihenfolge & -127.66 & 79.55 & -2.30 & -1.61 & .119 \\
\hline IE: RA $x$ Reihenfolge & 35.51 & 25.57 & 2.31 & 1.39 & .175 \\
\hline IE: RE $x$ Reihenfolge & -1.33 & 24.05 & -.09 & -.06 & .956 \\
\hline
\end{tabular}

Tabelle 37: Regressionsanalyse der $S^{(+)}$-Bedingungen zur Prüfung möglicher Reihenfolgeeffekte; mit der Investitionsbereitschaft als Kriteriumsvariable (vgl. Abschnitt 4.4 .2 - Model lb). 


\section{Analysen auf Basis des erweiterten Designs, wie in den Replikationsstudien:}

\begin{tabular}{|c|c|c|c|c|c|}
\hline \multirow{2}{*}{ Model } & \multicolumn{2}{|c|}{ Unstandardized Coefficients } & \multirow{2}{*}{$\begin{array}{c}\text { Standardized } \\
\text { Coefficients } \\
\text { Beta }\end{array}$} & \multirow{2}{*}{$\mathrm{t}$} & \multirow{2}{*}{ Sig. } \\
\hline & B & Std. Error & & & \\
\hline (Constant) & 141.90 & 65.61 & & 2.16 & .034 \\
\hline Verantwortlichkeit & 2.95 & 2.81 & .14 & 1.05 & .298 \\
\hline Alter & -2.19 & 1.34 & -.19 & -1.64 & .106 \\
\hline Geschlecht & 14.03 & 7.49 & .22 & 1.87 & .066 \\
\hline Framing & -13.24 & 6.88 & -.22 & -1.92 & .059 \\
\hline Sunk Cost & -31.29 & 60.87 & -.51 & -.51 & .609 \\
\hline$R T S$ & -15.27 & 16.74 & -.22 & -.91 & .365 \\
\hline$I E \_R T S \_S C$ & 8.21 & 17.27 & .50 & .48 & .636 \\
\hline Reihenfolge & -87.88 & 62.31 & -1.44 & -1.41 & .163 \\
\hline IE: RTS $x$ Reihenfolge & 18.98 & 17.64 & 1.14 & 1.08 & .286 \\
\hline IE: RTS $x$ SC $x$ Reihenfolge & 4.50 & 3.93 & .24 & 1.15 & .256 \\
\hline
\end{tabular}

Tabelle 38: Regressionsanalyse auf Basis des erweiterten Designs zur Prüfung möglicher Reihenfolgeeffekte; mit der Investitionsbereitschaft als Kriteriumsvariable (vgl. Abschnitt 4.4.2 - Model 2a).

\begin{tabular}{|c|c|c|c|c|c|}
\hline \multirow[b]{2}{*}{ Model } & \multicolumn{2}{|c|}{ Unstandardized Coefficients } & \multirow{2}{*}{$\begin{array}{c}\text { Standardized } \\
\text { Coefficients } \\
\text { Beta } \\
\end{array}$} & \multirow[b]{2}{*}{$\mathrm{t}$} & \multirow[b]{2}{*}{ Sig. } \\
\hline & $\mathrm{B}$ & Std. Error & & & \\
\hline (Constant) & 117.30 & 73.11 & & 1.60 & .114 \\
\hline Verantwortlichkeit & 2.99 & 2.96 & .14 & 1.01 & .316 \\
\hline Alter & -1.86 & 1.43 & -.16 & -1.30 & .198 \\
\hline Geschlecht & 13.47 & 7.72 & .21 & 1.74 & .086 \\
\hline Framing & -13.17 & 7.10 & -.22 & -1.86 & .068 \\
\hline Sunk Cost & -16.71 & 64.28 & -.27 & -.26 & .796 \\
\hline$R A$ & 9.17 & 22.13 & .14 & .41 & .680 \\
\hline$R E$ & -20.63 & 18.45 & -.36 & -1.12 & .268 \\
\hline$I E \_R A \_S C$ & -16.73 & 28.08 & -1.02 & -.60 & .553 \\
\hline$I E \_R E \_S C$ & 21.95 & 25.25 & 1.35 & .87 & .388 \\
\hline Reihenfolge & -80.03 & 66.31 & -1.31 & -1.21 & .232 \\
\hline IE: RA $x$ Reihenfolge & 2.52 & 28.35 & .15 & .09 & .930 \\
\hline IE: RE $x$ Reihenfolge & 15.00 & 24.92 & .90 & .60 & .550 \\
\hline IE: RA $x$ SC $\times$ Reihenfolge & 32.12 & 34.21 & 1.68 & .94 & .352 \\
\hline IE: RE x SC x Reihenfolge & -27.81 & 35.02 & -1.49 & -.79 & .430 \\
\hline
\end{tabular}

Tabelle 39: Regressionsanalyse auf Basis des erweiterten Designs zur Prüfung möglicher Reihenfolgeeffekte; mit der Investitionsbereitschaft als Kriteriumsvariable (vgl. Abschnitt 4.4 .2 - Model 2b). 